NATIONAL LABORATORY

MANAGED BY UT-BATTELLE

FOR THE DEPARTMENT OF ENERGY

\title{
Hot Water Distribution System Program Documentation and Comparison to Experimental Data
}

\section{September 2007}

\author{
Prepared by \\ Evelyn Baskin \\ GE Infrastructure Energy \\ William G. Craddick \\ Roberto Lenarduzzi \\ Robert L. Wendt \\ Oak Ridge National Laboratory \\ Keith A. Woodbury \\ University of Alabama
}

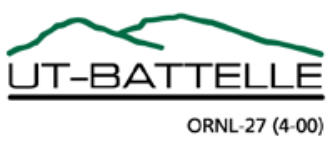




\section{DOCUMENT AVAILABILITY}

Reports produced after January 1, 1996, are generally available free via the U.S. Department of Energy (DOE) Information Bridge.

Web site http://www.osti.gov/bridge

Reports produced before January 1, 1996, may be purchased by members of the public from the following source.

National Technical Information Service

5285 Port Royal Road

Springfield, VA 22161

Telephone 703-605-6000 (1-800-553-6847)

TDD 703-487-4639

Fax 703-605-6900

E-mailinfo@ntis.gov

Web site http://www.ntis.gov/support/ordernowabout.htm

Reports are available to DOE employees, DOE contractors, Energy Technology Data Exchange (ETDE) representatives, and International Nuclear Information System (INIS) representatives from the following source.

Office of Scientific and Technical Information

P.O. Box 62

Oak Ridge, TN 37831

Telephone 865-576-8401

Fax 865-576-5728

E-mail reports@osti.gov

Web site http://www.osti.gov/contact.html

This report was prepared as an account of work sponsored by an agency of the United States Government. Neither the United States Government nor any agency thereof, nor any of their employees, makes any warranty, express or implied, or assumes any legal liability or responsibility for the accuracy, completeness, or usefulness of any information, apparatus, product, or process disclosed, or represents that its use would not infringe privately owned rights. Reference herein to any specific commercial product, process, or service by trade name, trademark, manufacturer, or otherwise, does not necessarily constitute or imply its endorsement, recommendation, or favoring by the United States Government or any agency thereof. The views and opinions of authors expressed herein do not necessarily state or reflect those of the United States Government or any agency thereof. 
ORNL/TM-2007/170

Engineering Science and Technology Division

\title{
HOT WATER DISTRIBUTION SYSTEM PROGRAM DOCUMENTATION AND COMPARISON TO EXPERIMENTAL DATA
}

\author{
Evelyn Baskin \\ GE Infrastructure Energy \\ William G. Craddick \\ Roberto Lenarduzzi \\ Robert L. Wendt \\ Oak Ridge National Laboratory
}

Keith A. Woodbury

University of Alabama

September 2007

Prepared by

Oak Ridge National Laboratory

Oak Ridge, Tennessee 37831-6285

managed by

UT-BATTELLE, LLC

for the

U.S. DEPARTMENT OF ENERGY

under contract DE-AC05-00OR22725 



\section{CONTENTS}

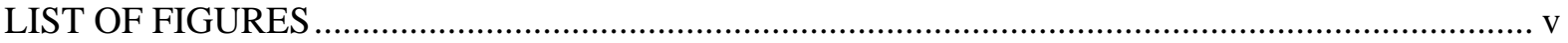

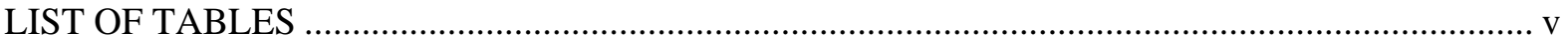

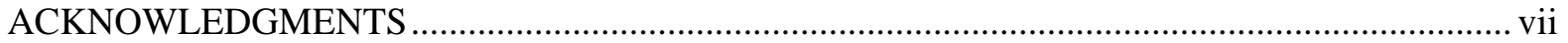

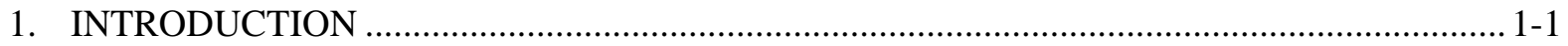

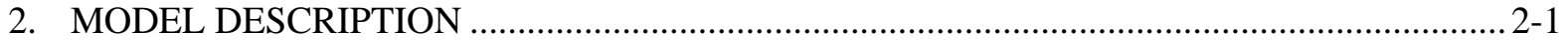

2.1 DESCRIPTION

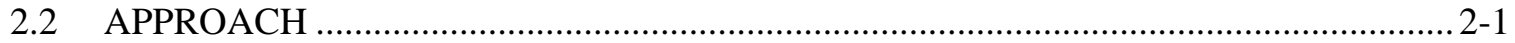

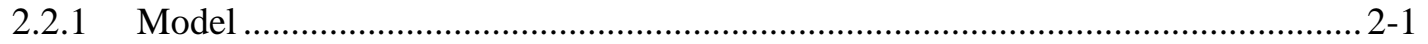

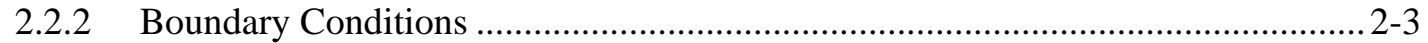

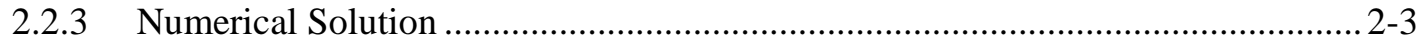

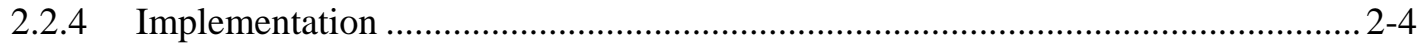

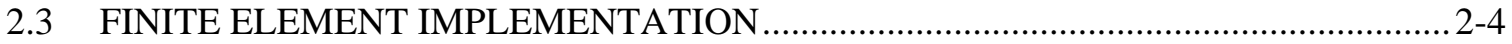

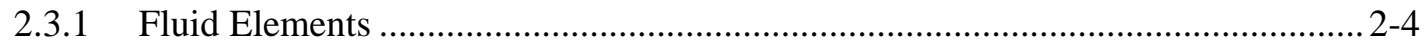

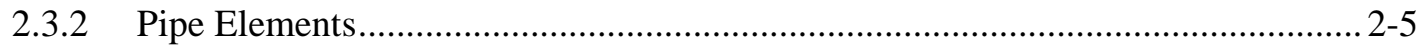

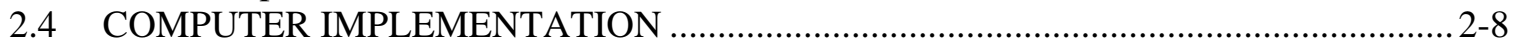

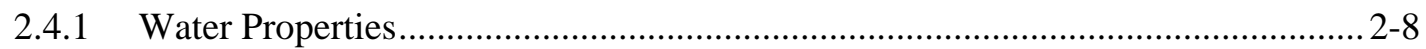

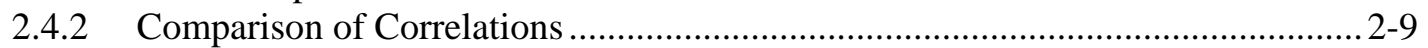

2.4.3 Steady State Fluid Element Solution................................................................ 2-10

2.4.4 Steady State Pipe Element Solution......................................................... 2-10

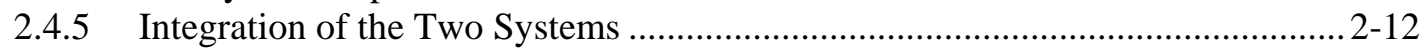

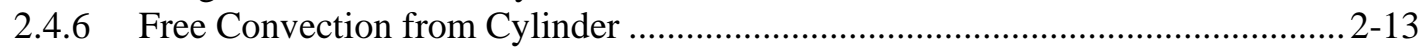

2.4.7 Forced Convection from Cylinder .................................................................. 2-13

2.4.8 Radiation from Cylinder ........................................................................ 2-13

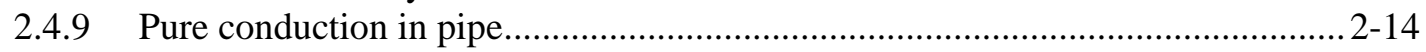

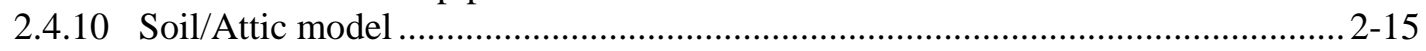

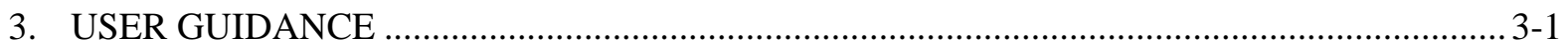

4. COMPARISONS TO EXPERIMENTAL DATA …........................................................... $4-1$

5. CONSIDERATIONS IN MODELING RESIDENTIAL SYSTEMS .......................................... 5-1

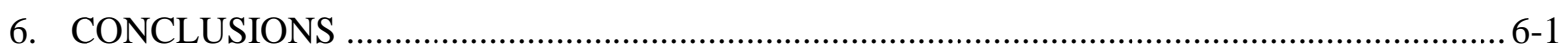

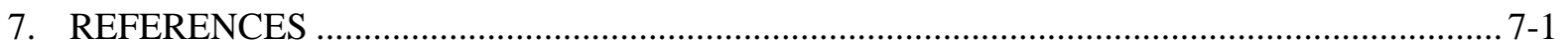

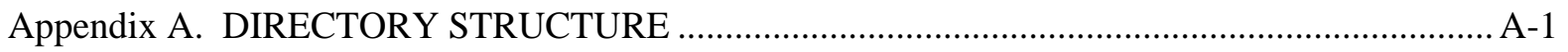

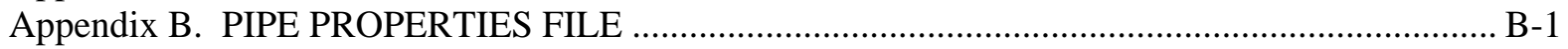

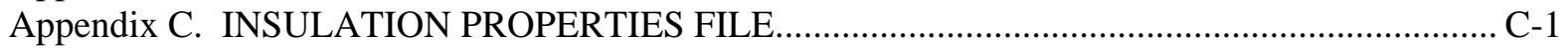

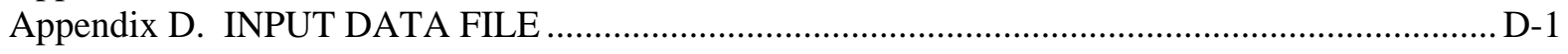

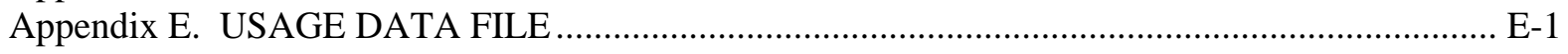

Appendix F. USE OF THE CORE CALCULATIONAL PROGRAM ............................................

Appendix G. ORNLpipe: PROGRAM DOCUMENTATION ……................................................... G-1

Appendix H. HWDS VALIDATION AND IMPROVEMENT ….................................................... 



\section{LIST OF FIGURES}

Figure

Page

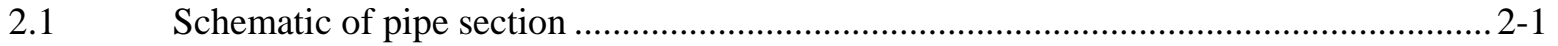

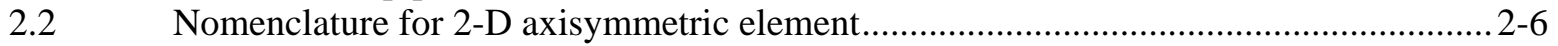

$2.3 \quad$ Computed heat transfer coefficient variations ............................................................. 2-10

2.4 Computed solution with variable properties compared with exact solution for constant properties ................................................................................................... 2-11

$2.5 \quad$ Radial temperature distribution for test case.............................................................. 2-11

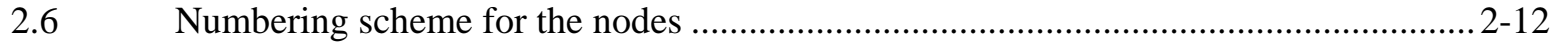

$2.7 \quad$ Test case for integrated model (fluid and pipe) .......................................................... 2-12

2.8 Convection and radiation exchange coefficients on pipe exterior ...............................2-14

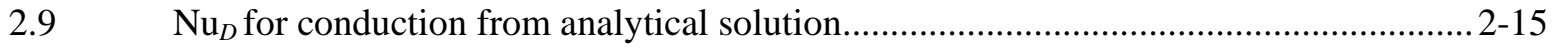

2.10 SOIL/ATTIC material of thickness thick surrounding pipe/insulation..........................2-16

3.1 ORNL-HWD Simulation main window .................................................................... 3-4

3.2 Simulation input data window (Step 2) for all cases except demand recirculation ..........3-5

Explorer window showing contents of InputData directory …..................................... 3-5

3.4 Explorer window showing contents of UsageData directory....................................... 3-6

3.5 Simulation input data window (Step 2) for demand recirculation case ......................... 3-7

3.6 Simulation Input Data window after selections completion .......................................... 3-7

3.7 ORNL-HWD Simulation main window after input data selection is completed..............3-8

3.8 ORNL-HWD Simulation main window while computations are in progress..................3-8

3.9 ORNL-HWD Simulation main window when computation is completed.......................3-9

3.10 Summary worksheet from results file for continuous recirculation case ...................... 3-10

3.11 Results worksheet from results file for conventional case ......................................... 3-11

3.12 Temperature worksheet from results file for conventional case .................................. 3-11

3.13 Demand worksheet from results file for demand recirculation case ............................. 3-12

3.14 Recirculation worksheet from results file for continuous recirculation case ..................3-12

G.1 Overall program flow in main() of RunPipeAllTrans.cppMain Program Flow..............G-1

G.2 Program flow in routine PipeAllTrans................................................................... G-9

\section{LIST OF TABLES}

Table

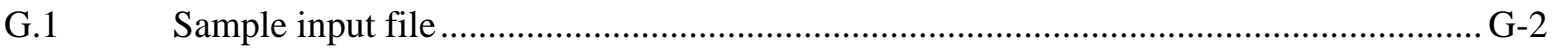





\section{ACKNOWLEDGMENTS}

This work was sponsored by the California Energy Commission through the Lawrence Berkley National Laboratory. 



\section{INTRODUCTION}

In 2003, the California Energy Commission's (CEC’s) Public Interest Energy Research (PIER) program funded Oak Ridge National Laboratory (ORNL) to create a computer program to analyze hot water distribution systems for single family residences, and to perform such analyses for a selection of houses. This effort and its results were documented in a report provided to CEC in March, 2004 [1]. The principal objective of effort was to compare the water and energy wasted between various possible hot water distribution systems for various different house designs. It was presumed that water being provided to a user would be considered suitably warm when it reached $105^{\circ} \mathrm{F}$. Therefore, what was needed was a tool which could compute the time it takes for water reaching the draw point to reach $105^{\circ} \mathrm{F}$, and the energy wasted during this wait.

The computer program used to perform the analyses was a combination of a calculational core, produced by Dr. Keith A. Woodbury, Professor of Mechanical Engineering and Director, Alabama Industrial Assessment Center, University of Alabama, and a user interface based on LabVIEW, created by Dr. Roberto Lenarduzzi of ORNL. At that time, the computer program was in a relatively rough and undocumented form-adequate to perform the contracted work but not in a condition where it could be readily used by those not involved in its generation.

Subsequently, the CEC provided funding through Lawrence Berkeley National Laboratory (LBNL) to improve the program's documentation and user interface to facilitate use by others, and to compare the program's results to experimental data generated by Dr. Carl Hiller. This report describes the program and provides user guidance. It also summarizes the comparisons made to experimental data, along with options built into the program specifically to allow these comparisons. These options were necessitated by the fact that some of the experimental data required options and features not originally included in the program. A more detailed description of these program modifications along with detailed comparisons to the experimental data are provided in a report produced by Dr. Woodbury, which accompanies this report as Appendix H.

The calibration of the model to experimental testing and review of the initial modeling methodology have resulted in a more accurate model that may no longer provide the same results for some situations as those documented in the earlier report.

Provided to CEC along with this report are the version of the program that includes the user interface, to facilitate general use, and the source code for the calculational core, which will allow others to compile the program using choices for compile-time options different than those selected for the version linked to the user interface. 



\section{MODEL DESCRIPTION}

The hot water distribution calculation package consists of two main components: the core calculation program, described here, and the user interface program, described in Sect. 3. The former was produced by Dr. Woodbury. It consists of $\mathrm{C}++$ code. This section describes the algorithms and equations used by the calculational program. Guidance on using the calculational program in standalone mode (without the user interface) is provided in Appendix F. Additional information on programming aspects is provided in Appendix G. After the initial program was created, an additional effort was undertaken to compare the program to experimental data, which led to some enhancements being made, most notably the inclusion of a gap conductance model. These enhancements, along with the comparisons to experimental data, are provided in a report produced by Dr. Woodbury and included as Appendix $\mathrm{H}$ of this document.

\subsection{DESCRIPTION}

A hot water distribution system consists of $n_{\text {seg }}$ segments of pipe of standard construction and optionally covered with insulation. The pipe segments may be exposed to either a convection environment with known conditions ( $h, T_{\text {inf }}$ ) or buried underground. The entire piping system is initially filled with water at the temperature of the surroundings, $T_{\text {surr }}$. Water at a known supply temperature $T_{\text {supply }}$ and mass flow rate $\dot{m}$ is continuously introduced into the piping system from an inexhaustible supply reservoir.

Find the temperature of the water along the pipe for an arbitrary time interval and, in particular, the temperature of water at the exit of the piping system (destination).

\subsection{APPROACH}

\subsubsection{Model}

This problem can be modeled as one dimensional energy transport in the axial direction of the piping system with lateral heat losses to the pipe wall. The temperature distribution in the pipe wall and insulation can be computed using two-dimensional calculations, coupled to the one-dimensional pipe solution through a heat transfer coefficient. A schematic of a pipe section indicating the nomenclature is shown in Fig. 2.1 below.

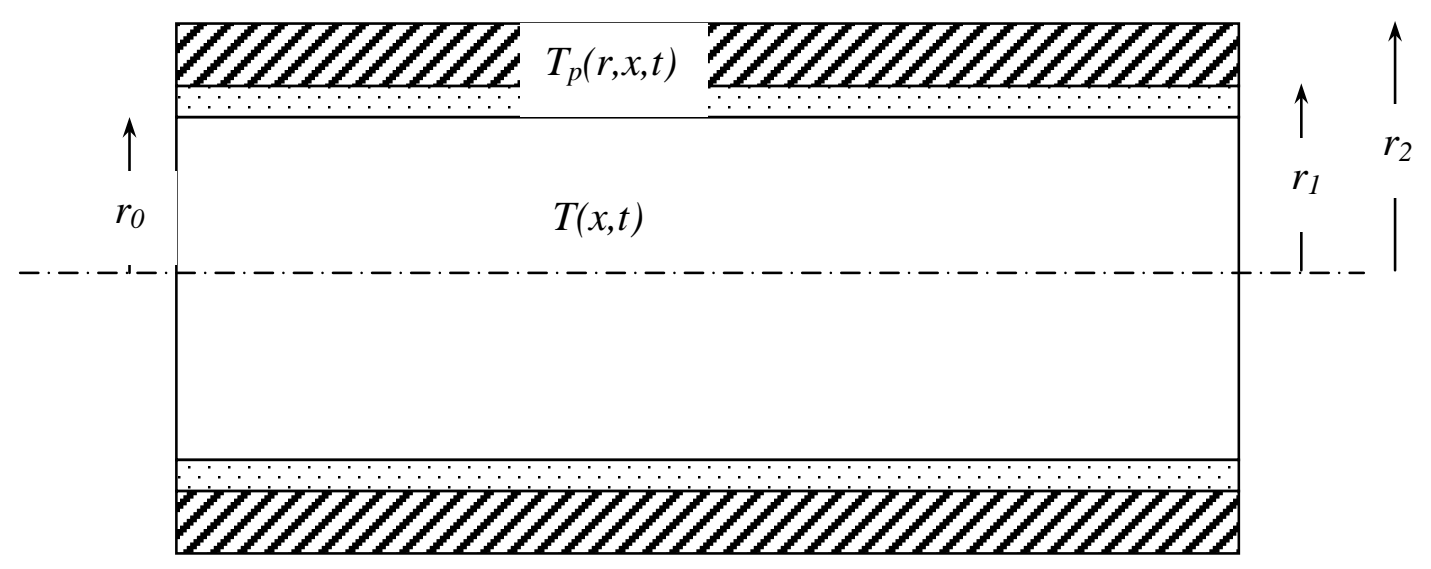

Fig. 2.1. Schematic of pipe section. 
Mathematically the problem can be described as follows. In the pipe, the (axial) temperature distribution will be governed by

$$
\dot{m} c_{p} \frac{\partial T}{\partial x}+\rho c_{p} A_{c s} \frac{\partial T}{\partial t}+p q_{\text {loss }}^{\prime \prime}=k A_{c s} \frac{\partial^{2} T}{\partial x^{2}}
$$

Here $p$ is the perimeter of the pipe, $A_{c s}$ is the cross sectional area, and $k, c_{p}$, and $\rho$ are properties of the fluid. The heat loss from the fluid to the pipe wall will be modeled via a heat transfer coefficient as

$$
q_{\text {loss }}^{\prime \prime}=h(x, t)\left(T(x, t)-T_{s}(x, t)\right)
$$

where $T_{s}(x, t)$ is the temperature of the surface of the pipe. The heat transfer coefficient can be computed from a conventional correlation for smooth pipes, such as the one given by ${ }^{1}$

$$
\mathrm{Nu}_{D}=\frac{(f / 8)\left(\mathrm{Re}_{D}-1000\right) \operatorname{Pr}}{1+12.7(f / 8)^{1 / 2}\left(\operatorname{Pr}^{2 / 3}-1\right)}
$$

which is valid for smooth tubes and Reynolds numbers in range $3000<\mathrm{Re}_{D}<5 \times 10^{6}$. In Eq. (3), the Moody friction factor $f$ can be computed from a correlation such as the Churchill relation: ${ }^{2}$

$$
\begin{aligned}
& f=8\left[\left(\frac{8}{\operatorname{Re}_{D}}\right)^{12}+\frac{1}{(A+B)^{1.5}}\right]^{1 / 12} \\
& A=\left\{2.457 \ln \left[\frac{1}{\left(7 / \operatorname{Re}_{D}\right)^{0.9}+(0.27 \varepsilon / D)}\right]\right\}^{16} \\
& B=\left(\frac{37530}{\operatorname{Re}_{D}}\right)^{16}
\end{aligned}
$$

In Eqs. (3) and (4), the properties of the fluid are evaluated at the temperature $T(x, t)$, thus the heat transfer coefficient $h(x, t)$ will be a weak function of the axial distance along the pipe.

The temperature distribution in the pipe and insulation can be computed from the solution of the twodimensional heat conduction equation in radial coordinates:

$$
\rho c_{p} \frac{\partial T_{p}}{\partial t}=\frac{1}{r} \frac{\partial}{\partial r}\left(k r \frac{\partial T_{p}}{\partial r}\right)+k \frac{\partial^{2} T_{p}}{\partial x^{2}}
$$

where the radial variation in $k$ must be retained (to allow for insulation over the pipe) but the axial variation in $k$ can be ignored. Here $T_{p}(r, x, t)$ is the solution for the temperature in the pipe and/or insulation, and the temperature $T_{s}(x, t)$ is Eq. (2) is simply the value of $T_{p}$ at the pipe inner radius:

$$
T_{s}(x, t)=T_{p}\left(r_{0}, x, t\right)
$$




\subsubsection{Boundary Conditions}

The boundary condition on Eq. (5) is an external convection environment with a known reference temperature:

$$
-\left.k \frac{\partial T}{\partial r}\right|_{r_{2}}=h\left(T_{p}\left(r_{2}, x, t\right)-T_{\infty}\right)
$$

while Eq. (2) is used to couple the solution for $T_{p}(r, x, t)$ to that for $T(x, t)$.

Suitable descriptions for approximating the boundary condition for a pipe buried in soil will be sought. Some possibilities:

1. Finite radius of soil with constant temperature at the periphery - this will be valid and useful if the simulation time of interest is not long enough to result in an appreciable temperature rise at the periphery.

2. Equivalent resistance of finite radius of soil—-this will yield results similar to above (with the same restriction) but ignores the heat capacity of the soil.

3. Conduction shape factor to surface - can be used for a shallowly buried pipe but will account for only part of the energy loss.

\subsubsection{Numerical Solution}

The equations (1)-(7) can be solved by any appropriate numerical scheme. The Finite Element method will be used in this development. Standard linear interpolation elements will be used for the axial temperature $T(x)$ and bilinear rectangular elements will be used to represent $T_{p}(r, x)$.

Following the Galerkin Method of Weighted Residuals development for finite elements, ${ }^{3}$ the discrete representation of Eq. (1) is

$$
[C]\{\dot{T}\}+\left[K_{m}+K_{c}+K_{h}\right]\{T\}=\left\{R_{h}\right\}
$$

where $K_{m}, K_{c}, K_{h}$ are the contributions from the mass flow term, the conduction term, and the $q_{\text {loss }}$ term, respectively, and the $R_{h}$ is the thermal load imposed associated with the $q_{\text {loss }}$ term. The discrete representation of Eq. (5) is

$$
[C]\left\{\dot{T}_{p}\right\}+\left[K_{c}+K_{h}+K_{h_{2}}\right]\left\{T_{p}\right\}=\left\{R_{h}+R_{h_{2}}\right\}
$$

where the matrices have similar meaning, and the ' $h_{2}$ ' terms are associated with the external convection environment.

The contributions from all the elements are combined into a system matrix for all the temperatures. This can be expressed symbolically as:

$$
\left[C_{\text {sys }}\right]\left\{\dot{T}_{\text {all }}\right\}+\left[K_{\text {sys }}\right]\left\{T_{\text {all }}\right\}=\left\{R_{\text {sys }}\right\}
$$

This set of coupled, first order differential equations can be solved recursively by using a generalized Crank-Nicholson scheme, which results in an implicit set of algebraic equations: 


$$
\begin{aligned}
{\left[\theta\left[K_{\text {sys }}\right]+\frac{1}{\Delta t}\left[C_{\text {sys }}\right]\right]\left\{T_{\text {all }}\right\}_{n+1}=} & {\left[-(1-\theta)\left[K_{\text {sys }}\right]+\frac{1}{\Delta t}\left[C_{\text {sys }}\right]\right]\left\{T_{\text {all }}\right\}_{n}+} \\
& (1-\theta)\left\{R_{\text {sys }}\right\}_{n}+\theta\left\{R_{s y s}\right\}_{n+1}
\end{aligned}
$$

Here $\theta$ is the integration parameter: for $\theta=1 / 2=0.5$ the unconditionally stable Crank-Nicholson scheme results; for $\theta=2 / 3$ the unconditionally stable Galerkin procedure results.

\subsubsection{Implementation}

The numerical equations described above will be incorporated into a group of MatLAB functions. MatLAB offers a comfortable environment for matrix operations, providing a number of intrinsic features that greatly simplify the programming effort.

\subsection{FINITE ELEMENT IMPLEMENTATION}

A standard Galerkin Method of Weighted Residuals technique is used to derive the element equations. An interpolation function is used over each element to represent the temperature, and this interpolation function is used as the weight function to force the residual of the governing differential equation in a weighted sense. Integration by parts is used on the highest order term in the equation to lessen the differentiability requirement of the interpolation functions. See [3] for more details on the method.

\subsubsection{Fluid Elements}

The temperature distribution in the fluid can be considered one-dimensional in the direction of flow. The computed temperature is then the bulk (thermodynamic) temperature of the fluid. A lateral heat loss for these one-dimensional elements will be implemented by linking the temperature at each node to that of the surrounding pipe will with a "gap" element [viz. Eq. (2)].

For the one-dimensional elements, a linear temperature distribution is assumed:

$$
\begin{aligned}
T^{e}(x, t) & =\left\lfloor 1-x / \ell_{e} \quad x / \ell_{e}\right\rfloor\left\{\begin{array}{c}
T_{1}^{e}(t) \\
T_{2}^{e}(t)
\end{array}\right\} \\
& =\left\lfloor N_{1}(x) \quad N_{2}(x)\right\rfloor\left\{T^{e}(t)\right\}
\end{aligned}
$$

When this variation is substituted into the governing Eq. (1), that equation will not, in general, be satisfied: a residual will remain. This residual is weighted over the element with the interpolation functions (one for each node) and the following matrix weighted residual statement results:

$$
\begin{aligned}
\int_{0}^{\ell_{e}} \dot{m} c_{p}\{N\}\left\lfloor\frac{d N}{d x}\right\rfloor d x\left\{T^{e}(t)\right\}+ & \int_{0}^{\ell_{e}} \rho c_{p} A_{c s}\{N\}\lfloor N\rfloor d x\left\{\dot{T}^{e}\right\}+ \\
& \int_{0}^{\ell_{e}} p h(x, t)\{N\}\left(\lfloor N\rfloor\left\{T^{e}(t)\right\}-T_{s}(x, t)\right) d x=\int_{0}^{\ell_{e}}\{N\} k A_{c s} \frac{\partial^{2} T}{\partial x^{2}} d x
\end{aligned}
$$

The term on the right is integrated by parts, giving 


$$
\begin{aligned}
\int_{0}^{\ell_{e}} \dot{m} c_{p}\{N\}\left\lfloor\frac{d N}{d x}\right\rfloor d x\left\{T^{e}(t)\right\}+\int_{0}^{\ell_{e}} \rho c_{p} A_{c s}\{N\}\lfloor N\rfloor d x\left\{\dot{T}^{e}\right\}+ \\
\int_{0}^{\ell_{e}} p h(x, t)\{N\}\left(\lfloor N\rfloor\left\{T^{e}(t)\right\}-T_{s}(x, t)\right) d x=\left\{\begin{array}{c}
\left.q_{i n}\right|_{0} \\
\left.q_{i n}\right|_{\ell_{e}}
\end{array}\right\}-\int_{0}^{\ell_{e}} k A_{c s}\left\{\frac{d N}{d x}\right\}\left\lfloor\frac{d N}{d x}\right\rfloor d x\left\{T^{e}(t)\right\}
\end{aligned}
$$

The mass flow rate is constant through the entire system; $c_{\mathrm{p}}$ and $k$ are assumed to be constant over each element. The heat transfer coefficient varies axially, but can be assumed constant over each element at every instant in time. Eq. (14) reduces to

$$
[C]\left\{\dot{T}^{e}\right\}+\left[\left[K_{m}\right]+\left[K_{h}\right]+\left[K_{c}\right]\right]\left\{T^{e}(t)\right\}=\left\{R_{q}\right\}+\left\{R_{h}\right\}
$$

where

$$
\begin{aligned}
& {[C]=\rho c_{p} A_{c s} \int_{0}^{\ell_{e}}\{N\}\lfloor N\rfloor d x=\frac{\rho c_{p} A_{c s} \ell_{e}}{6}\left[\begin{array}{ll}
2 & 1 \\
1 & 2
\end{array}\right]} \\
& {\left[K_{m}\right]=\dot{m} c_{p} \int_{0}^{\ell_{e}}\{N\}\left\lfloor\frac{d N}{d x}\right\rfloor d x=\frac{\dot{m} c_{p}}{2}\left[\begin{array}{ll}
-1 & 1 \\
-1 & 1
\end{array}\right]} \\
& {\left[K_{h}\right]=\int_{0}^{\ell_{e}} p h(x, t)\{N\}\lfloor N\rfloor\left\{T^{e}(t)\right\} d x=p \frac{h_{\text {ele }} \ell_{e}}{6}\left[\begin{array}{ll}
2 & 1 \\
1 & 2
\end{array}\right]} \\
& {\left[K_{c}\right]=\int_{0}^{\ell_{e}} k A_{c s}\left\{\frac{d N}{d x}\right\}\left\lfloor\frac{d N}{d x}\right\rfloor d x=\frac{k A_{c s}}{\ell_{e}}\left[\begin{array}{rr}
1 & -1 \\
-1 & 1
\end{array}\right]} \\
& \left\{R_{q}\right\}=\left\{\begin{array}{c}
\left.q_{i n}\right|_{0} \\
\left.q_{i n}\right|_{\ell_{e}}
\end{array}\right\} \\
& \left\{R_{h}\right\}=\int_{0}^{\ell_{e}} p h(x, t)\{N\} T_{s}(x, t) d x \\
& =\int_{0}^{\ell_{e}} p h_{\text {ele }}\{N\}\lfloor N\rfloor\left\{\begin{array}{l}
T_{s 1} \\
T_{s 2}
\end{array}\right\} d x \\
& =p \frac{h_{e l e} \ell_{e}}{6}\left[\begin{array}{ll}
2 & 1 \\
1 & 2
\end{array}\right]\left\{\begin{array}{l}
T_{s 1} \\
T_{s 2}
\end{array}\right\} \\
& =\left[K_{h}\right]\left\{\begin{array}{l}
T_{s 1} \\
T_{s 2}
\end{array}\right\}
\end{aligned}
$$

\subsubsection{Pipe Elements}

The heat conduction equation in cylindrical coordinates can be written as

$$
\nabla \cdot \vec{q}^{\prime \prime}+\rho c_{p} \frac{\partial T}{\partial t}=0
$$


The weighted residual statement for an element is formed by multiplying by one of the interpolation functions and integrating over the area (volume) of the element:

$$
\int_{\Omega^{e}} N_{i}\left(\nabla \cdot \vec{q}^{\prime \prime}\right) d \Omega+\int_{\Omega^{e}} N_{i}\left(\rho c_{p} \frac{\partial T}{\partial t}\right) d \Omega=0
$$

Apply Green's theorem to the first term

$$
\int_{\Gamma^{e}} N_{i}\left(\vec{q}^{\prime \prime} \cdot \hat{n}\right) d \Gamma-\int_{\Omega^{e}} \nabla N_{i} \cdot \vec{q}^{\prime \prime} d \Omega+\int_{\Omega^{e}} N_{i}\left(\rho c_{p} \frac{\partial T}{\partial t}\right) d \Omega=0
$$

The term in parenthesis in the line integral is the heat flow out of the element. If the term is moved to the right side of the equation it is the heat flow into the element. Also, the heat flux vector is given by the Fourier Law $\vec{q}^{\prime \prime}=-k \nabla T$, so Eq. (18) can be written as

$$
\int_{\Omega^{e}} N_{i}\left(\rho c_{p} \frac{\partial T}{\partial t}\right) d \Omega+\int_{\Omega^{e}} \nabla N_{i} \cdot(k \nabla T) d \Omega+=\int_{\Gamma^{e}} N_{i}\left(q_{i n}\right) d \Gamma
$$

For the two dimensional elements, a simple bi-linear interpolation function will be used on each element:

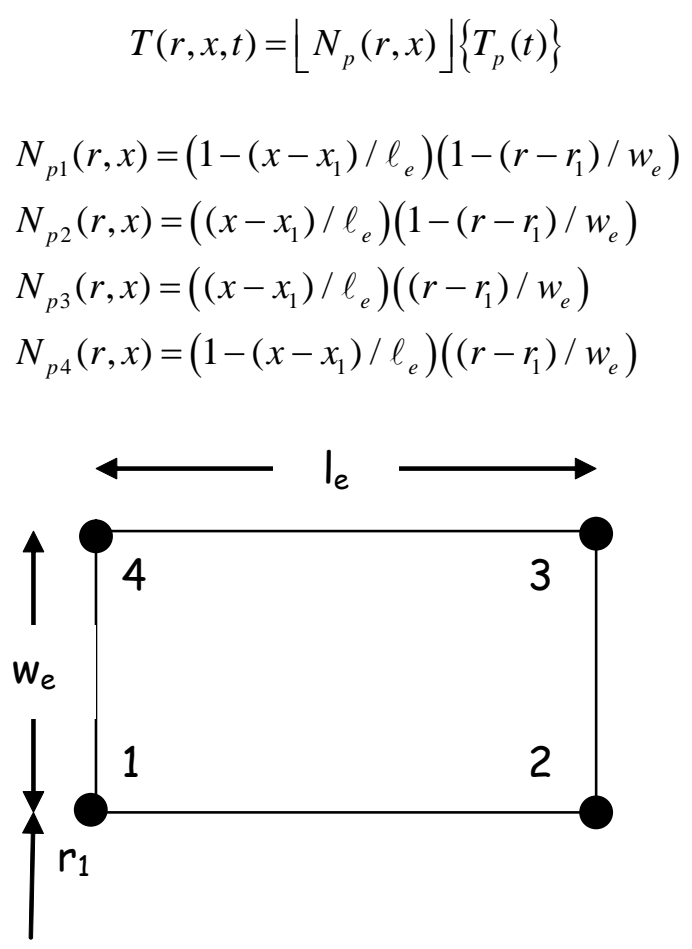

Fig. 2.2. Nomenclature for 2-D axisymmetric element. 
Define the global derivative operator $\mathbf{B}$ as

$$
\mathbf{B}=\left[\begin{array}{ll}
\frac{\partial N_{i}}{\partial r} & \frac{\partial N_{i}}{\partial x}
\end{array}\right]^{T}
$$

and writing Eq. (19) for all four nodes ( $i=1,2,3$, 4), with the area integral $d \Omega=2 \pi r d r d x$

$$
2 \pi \int_{\Omega^{e}} \rho c_{p}\left\{N_{p}\right\}\left\lfloor N_{p}\right\rfloor r d r d x\left\{\dot{T}_{p}\right\}+2 \pi \int_{\Omega^{e}} k \mathbf{B}^{T} \mathbf{B} \operatorname{rdr} d x\left\{T_{p}\right\}=\int_{\Gamma^{e}}\left\{N_{p}\right\}\left(q_{i n}\right) d \Gamma
$$

In order to handle the $r$ in the integrand, we follow the suggestion of Pepper and Heinrich ${ }^{4}$ regarding axisymmetric problems by interpolating the radial distance $r$ using the same basis functions as for the field variables. In this case, the $r$ obviously doesn't have any $x$ dependence, so the one-dimensional version of the interpolation functions can be used:

$$
r^{e}=\left\lfloor 1-\left(r-r_{1}\right) / w_{e} \quad\left(r-r_{1}\right) / w_{e}\right\rfloor\left\{\begin{array}{l}
r_{1} \\
r_{2}
\end{array}\right\}
$$

Then Eq. (22) can be written as

$$
\left[C_{p}\right]\left\{\dot{T}_{p}\right\}+\left[K_{p}\right]\left\{T_{p}(t)\right\}=\left\{R_{p}\right\} \quad q
$$

where

$$
\begin{aligned}
& {\left[C_{p}\right]=\rho c_{p} 2 \pi \int_{r_{1}}^{r_{2} \ell_{e}}\left\{N_{p}\right\}\left\lfloor N_{p}\right\rfloor r^{e} d r d x} \\
& =\frac{2 \pi \rho c_{p} \ell_{e} w_{e}}{72}\left[\begin{array}{cccc}
6 r_{1}+2 r_{2} & 3 r_{1}+r_{2} & r_{1}+r_{2} & 2\left(r_{1}+r_{2}\right) \\
& 6 r_{1}+2 r_{2} & 2\left(r_{1}+r_{2}\right) & r_{1}+r_{2} \\
& & 2 r_{1}+6 r_{2} & r_{1}+3 r_{2} \\
\text { sym } & & & 2 r_{1}+6 r_{2}
\end{array}\right] \\
& {\left[K_{p}\right]=2 \pi \int_{r_{1}}^{r_{2} \ell_{e}} \int_{0} k \mathbf{B}^{T} \mathbf{B} r^{e} d r d x} \\
& =\frac{2 \pi k_{e}}{12 \ell_{e} w_{e}}\left[\begin{array}{cc}
3 r_{1} w_{e}^{2}+2 r_{2} \ell_{e}^{2}+2 r_{1} \ell_{e}^{2}+r_{2} w_{e}^{2} & -3 r_{1} w_{e}^{2}+r_{2} \ell_{e}^{2}+r_{1} \ell_{e}^{2}-r_{2} w_{e}^{2} \\
3 r_{1} w_{e}^{2}+2 r_{2} \ell_{e}^{2}+2 r_{1} \ell_{e}^{2}+r_{2} w_{e}^{2} \\
s y m
\end{array}\right. \\
& -\left(\ell_{e}^{2}+w_{e}^{2}\right)\left(r_{1}+r_{2}\right) \quad\left(w_{e}^{2}-2 \ell_{e}^{2}\right)\left(r_{1}+r_{2}\right) \\
& \text {.. }\left(w_{e}^{2}-2 \ell_{e}^{2}\right)\left(r_{1}+r_{2}\right) \quad-\left(\ell_{e}^{2}+w_{e}^{2}\right)\left(r_{1}+r_{2}\right) \\
& r_{1} w_{e}^{2}+3 r_{2} w_{e}^{2}+2 r_{1} \ell_{e}^{2}+2 r_{2} \ell_{e}^{2} \quad-r_{1} w_{e}^{2}-3 r_{2} w_{e}^{2}+r_{1} \ell_{e}^{2}+r_{2} \ell_{e}^{2} \\
& r_{1} w_{e}^{2}+3 r_{2} w_{e}^{2}+2 r_{1} \ell_{e}^{2}+2 r_{2} \ell_{e}^{2}
\end{aligned}
$$


If, at the inner pipe boundary, the heat flux from the fluid to the pipe is described by the Eq. (2), then there are two terms arising similar to Eq. (15c) and Eq. (15f):

$$
\begin{aligned}
& {\left[K_{h}\right]=\int_{0}^{\ell_{e}} p h(x, t)\{N\}\lfloor N]\left\{T^{e}(t)\right\} d x=p \frac{h_{e l e} \ell_{e}}{6}\left[\begin{array}{cccc}
2 & 1 & 0 & 0 \\
1 & 2 & 0 & 0 \\
0 & 0 & 0 & 0 \\
0 & 0 & 0 & 0
\end{array}\right] } \\
&\left\{R_{h}\right\}=\int_{0}^{\ell_{e}} p h(x, t)\{N\} T_{f}(x, t) d x \\
&=p \frac{h_{e l e} \ell_{e}}{6}\left[\begin{array}{llll}
2 & 1 & 0 & 0 \\
1 & 2 & 0 & 0 \\
0 & 0 & 0 & 0 \\
0 & 0 & 0 & 0
\end{array}\right]\left\{\begin{array}{c}
T_{f 1} \\
T_{f 2} \\
0 \\
0
\end{array}\right\}=\left[K_{h}\right]\left[\begin{array}{c}
T_{f 1} \\
T_{f 2} \\
0 \\
0
\end{array}\right\}
\end{aligned}
$$

In Eq. (24d), the $T_{f 1}$ and $T_{f 2}$ are the temperatures of the fluid corresponding to the nodes 1 and 2 for the two-dimensional element, and $T_{f}(x, t)$ has been interpolated linearly along the length of the element.

For the outer boundary, a type 3 condition, similar to Eqs. (24c-d), is given

$$
\begin{aligned}
& {\left[K_{\text {ho }}\right]=\int_{0}^{\ell_{e}} p h_{o}(x, t)\{N\}\lfloor N\rfloor\left\{T^{e}(t)\right\} d x=p \frac{h_{o_{-} e l e} \ell_{e}}{6}\left[\begin{array}{llll}
0 & 0 & 0 & 0 \\
0 & 0 & 0 & 0 \\
0 & 0 & 2 & 1 \\
0 & 0 & 1 & 2
\end{array}\right]} \\
& \left\{R_{q h}\right\}=\int_{0}^{\ell_{e}} p h_{o}(x, t)\{N\} T_{\infty}(x, t) d x \\
& p \frac{h_{o_{-} e l e} \ell_{e}}{6}\left[\begin{array}{cccc}
0 & 0 & 0 & 0 \\
0 & 0 & 0 & 0 \\
0 & 0 & 2 & 1 \\
0 & 0 & 1 & 2
\end{array}\right]\left\{\begin{array}{c}
0 \\
0 \\
T_{\infty 1} \\
T_{\infty 2}
\end{array}\right\}
\end{aligned}
$$

\subsection{COMPUTER IMPLEMENTATION}

\subsubsection{Water Properties}

Properties of water are needed as a function of temperature for the computer program. Properties of water in English Units were taken from Table B-2 of Hodge and Taylor ${ }^{2}$ and Microsoft Excel was used to determine a suitable polynomial curve fit for each property. The relations obtained are:

$$
\begin{gathered}
\mu=1.565088 E-17 T^{6}-2.025391 E-14 T^{5}+1.067447 E-11 T^{4}-2.968080 E-09 T^{3} \\
+4.729008 E-07 T^{2}-4.385665 E-05 T+2.204072 E-03
\end{gathered}
$$




$$
\begin{gathered}
\operatorname{Pr}=4.371165 E-13 T^{6}-4.952243 E-10 T^{5}+2.296428 E-07 T^{4}-5.646478 E-05 T^{3} \\
\quad+7.978822 E-03 T^{2}-6.525279 E-01 T+2.802356 E+01
\end{gathered} \begin{gathered}
\rho=-5.3636232 E-05 T^{2}-8.7278764 E-04 T+6.2547878 E+01 \\
k=-4.129655 E-12 T^{4}+5.873309 E-09 T^{3}-3.865481 E-06 T^{2} \\
\quad+1.105924 E-03 T+2.865951 E-01
\end{gathered}
$$

For these relations, temperature $T$ is in degrees Fahrenheit. The percentage errors in these polynomial approximations are given in the table below.

\begin{tabular}{cccccc}
$\begin{array}{c}\text { Temperature } \\
\text { range }\end{array}$ & $\begin{array}{c}\boldsymbol{\rho}, \\
\mathbf{l b m} / \mathbf{f t}^{3}\end{array}$ & $\begin{array}{c}\mathbf{c}_{\mathbf{p}}, \\
\mathbf{B t u} / \mathbf{l b m} /{ }^{\circ} \mathbf{F}\end{array}$ & $\begin{array}{c}\mathbf{k}, \\
\mathbf{B t u} / \mathbf{h r} / \mathbf{f t} /{ }^{\circ} \mathbf{F}\end{array}$ & $\begin{array}{c}\boldsymbol{\mu}, \\
\mathbf{l b m} / \mathbf{f t} / \mathbf{s}\end{array}$ & $\begin{array}{c}\mathbf{P r}, \\
(-)\end{array}$ \\
\hline $32^{\circ} \mathrm{F}-300^{\circ} \mathrm{F}$ & $+0.13 \%$ & $+0.46 \%$ & $+0.25 \%$ & $+0.64 \%$ & $+0.41 \%$ \\
& $-0.29 \%$ & $-0.75 \%$ & $-0.28 \%$ & $-0.58 \%$ & $-0.52 \%$ \\
$60^{\circ} \mathrm{F}-150^{\circ} \mathrm{F}$ & $+0.13 \%$ & $+0.46 \%$ & $+0.20 \%$ & $+0.35 \%$ & $+0.41 \%$ \\
& $-0.00 \%$ & $-0.04 \%$ & $-0.07 \%$ & $-0.41 \%$ & $-0.52 \%$ \\
\hline
\end{tabular}

\subsubsection{Comparison of Correlations}

The correlation of Gnielinski for the heat transfer coefficient $h$ given by Eq. (3) is recommended for lower Reynolds numbers. A simple calculation using water properties at $100^{\circ} \mathrm{F}$ shows that the range of Reynolds numbers for flow rates of $0.5-4.0$ GPM in pipes $0.25 "-0.75$ " diameter is 3000-75000. A comparison of the Gnielinski correlation for water at $100^{\circ} \mathrm{F}$ to the correlation of Petukhov and to the Chilton-Colburn analogy and to the Dittus-Boelter relation ${ }^{1}$ is shown in the figure below.

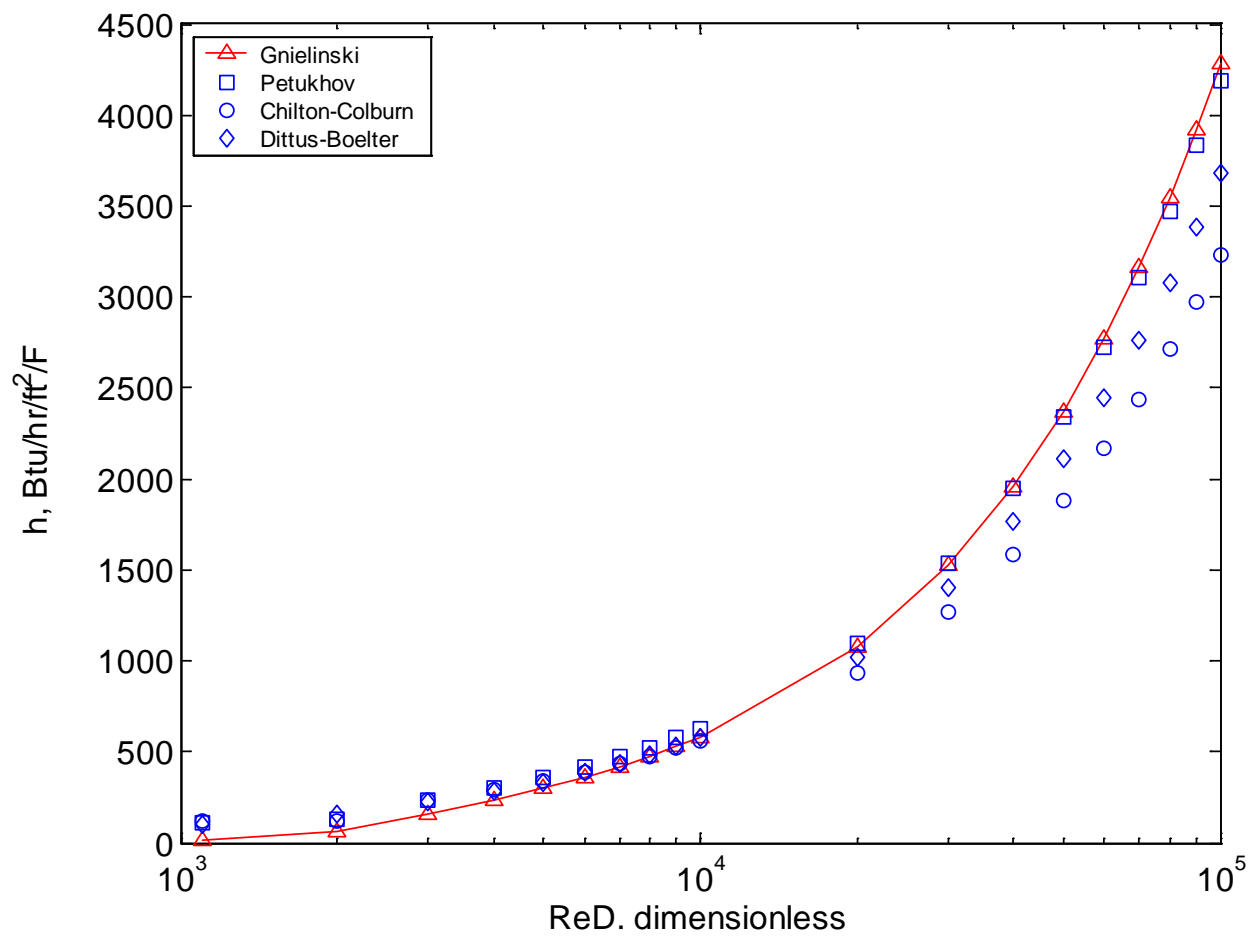




\subsubsection{Steady State Fluid Element Solution}

The one-dimensional solution for the steady temperature distribution in the fluid elements represented by Eq. (15) with the matrix [C] set to zero was obtained with a constant pipe wall temperature. A flow rate of about $1.5 \mathrm{GPM}(0.2 \mathrm{lbm} / \mathrm{s})$ was introduced into the 0.5 " diameter pipe 20 feet long with a constant pipe wall temperature of $100^{\circ} \mathrm{F}$, and the program iterated until the steady temperature distribution along the pipe was found. Due to the variation in fluid properties with temperature, a variation in heat transfer coefficient resulted. This variation in heat transfer coefficient is shown in Fig. 2.3 below, along with the average heat transfer coefficient.

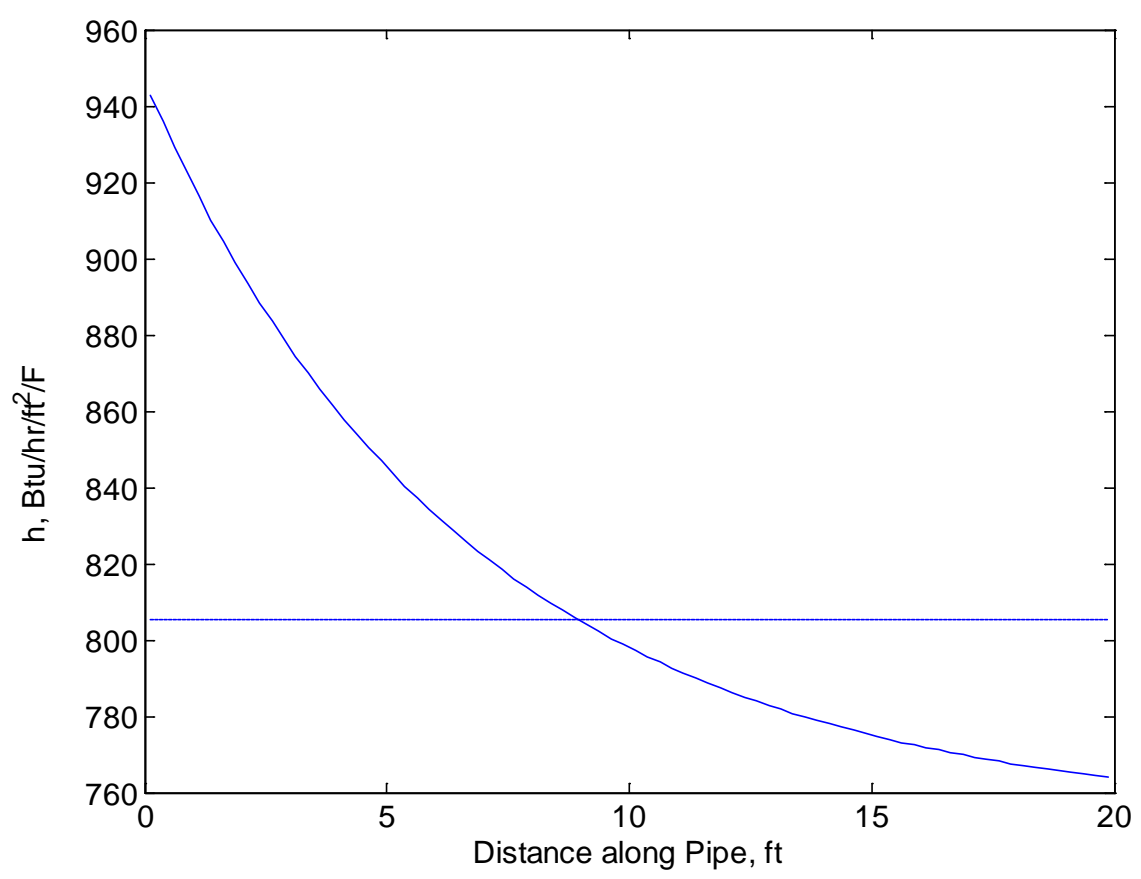

Fig. 2.3. Computed heat transfer coefficient variations.

An exact solution for the temperature distribution in a pipe with a constant wall temperature and constant heat transfer coefficient is widely known: ${ }^{1,2}$

$$
\frac{T_{s}-T(x)}{T_{s}-T_{i}}=\exp \left(\frac{-h p x}{\dot{m} c_{p}}\right)
$$

This solution is plotted with that from the 1-D steady finite element solution in Fig. 2.4 below. Also shown in the figure as a dashed red line is the FEM solution with a constant value of heat transfer coefficient along the pipe. The comparison with the exact solution is excellent.

\subsubsection{Steady State Pipe Element Solution}

The steady two-dimensional system represented by Eqs. (24) with [C] was solved using a constant internal pipe temperature of $100^{\circ} \mathrm{F}$ and heat transfer coefficient of $10 \mathrm{Btu} / \mathrm{s} / \mathrm{ft}^{2} /{ }^{\circ} \mathrm{F}$. Constant exterior conditions of $65^{\circ} \mathrm{F}$ and $1 \mathrm{Btu} / \mathrm{s} / \mathrm{ft}^{2} /{ }^{\circ} \mathrm{F}$ were also assumed. For this purpose a thick pipe with $0.5 "$ inside radius and 1.0" outside radius was assumed with constant conductivity $1 \mathrm{Btu} / \mathrm{hr} / \mathrm{ft} /{ }^{\circ} \mathrm{F}$. A short pipe 


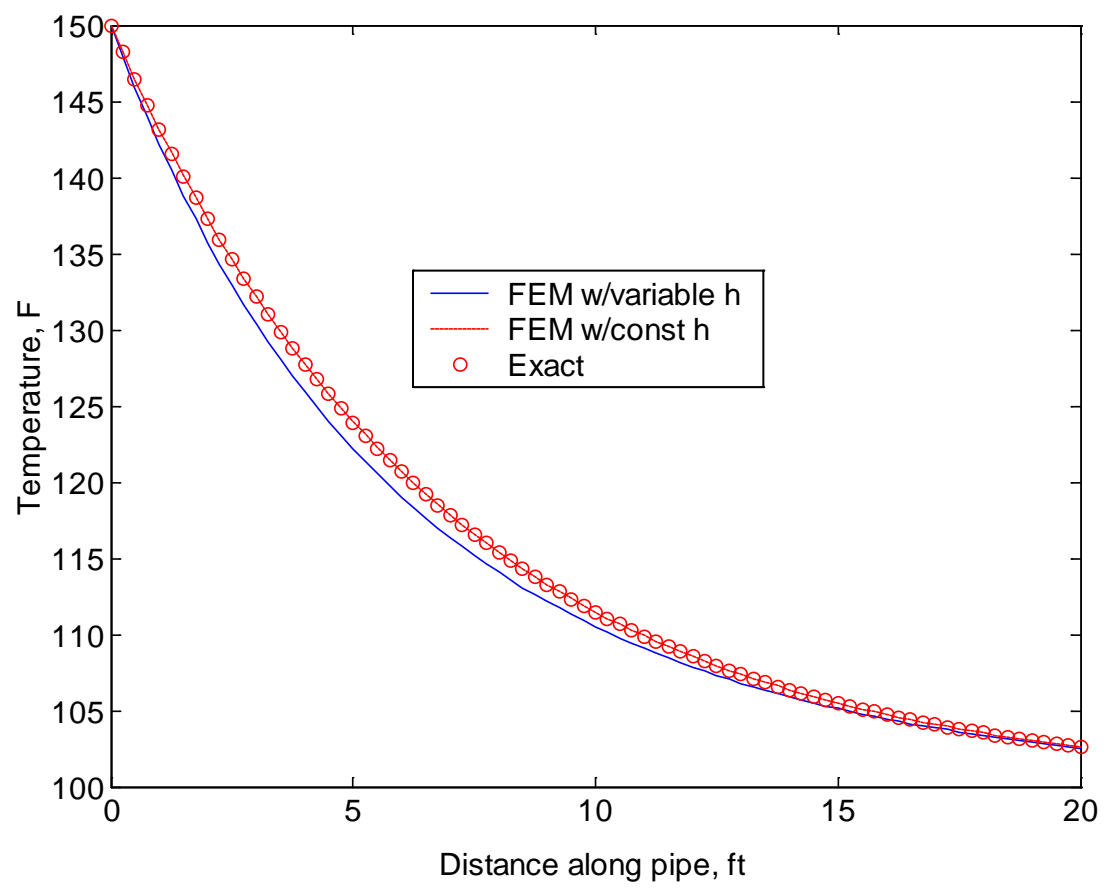

Fig. 2.4. Computed solution with variable properties compared with exact solution for constant properties.

length of 2 feet was used. The computation correctly produced a solution that is the same at each axial location. A plot of the predicted radial distribution of temperature in the pipe compared to the exact solution for this simple case is shown in Fig. 2.5 below.

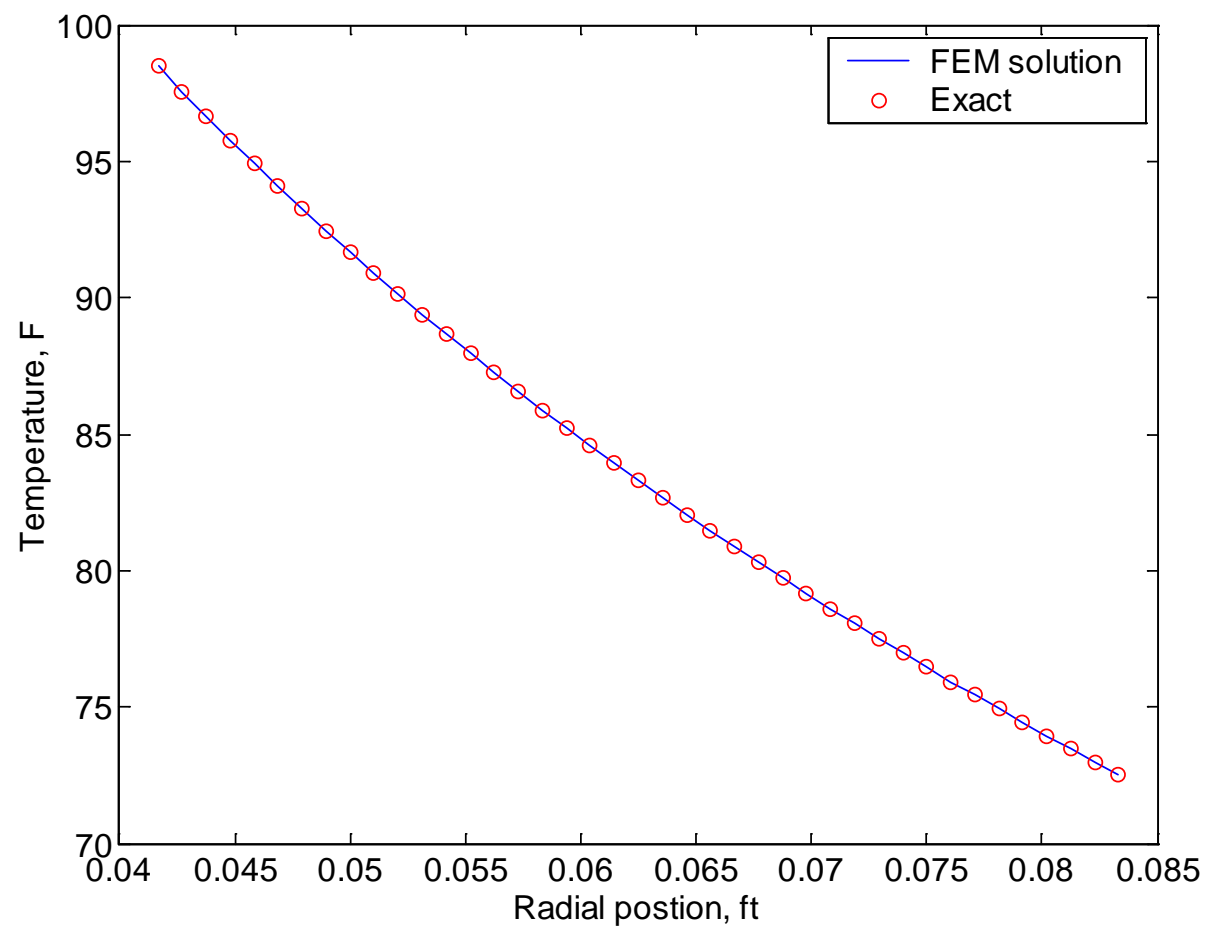

Fig. 2.5. Radial temperature distribution for test case. 


\subsubsection{Integration of the Two Systems}

Although the solutions for the pipe fluid and pipe wall have been developed and tested separately, we want to integrate these two analyses into a single computation to compute the whole temperature field at once. This is necessary to compute the transient response.

The numbering scheme for the nodes is shown in Fig. 2.6. There are $n_{r}$ nodes in the radial direction, and this is the direction for global numbering, as shown in Fig. 2.6. With $n_{x}$ nodes in the axial direction, there are a total of $n_{r} \times n_{x}$ nodes.

The integration of the two sets of equations into one global system can be accomplished easily in the finite element context by moving the $\left\{R_{h}\right\}$ terms from Eq. (15f) and Eq. (24d) onto the left side of their respective equations.

A test case with $0.2 \mathrm{lbm} / \mathrm{s}$ water at $150^{\circ} \mathrm{F}$ entering a 0.5 inch diameter copper pipe was used. A constant environment temperature of $65^{\circ} \mathrm{F}$ with $h_{o}=1.0 \mathrm{Btu} / \mathrm{s} / \mathrm{ft}^{2} /{ }^{\circ} \mathrm{F}$ was used. The steady state results are shown in Fig. 2.7. The exact solution for this case is that for fluid flowing in a pipe with constant

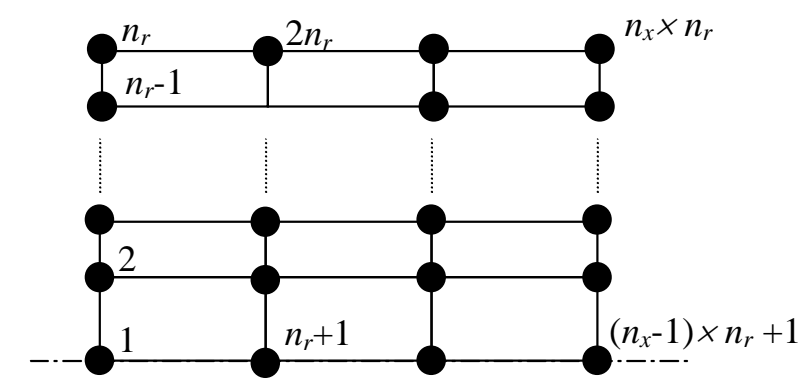

Fig. 2.6. Numbering scheme for the nodes.

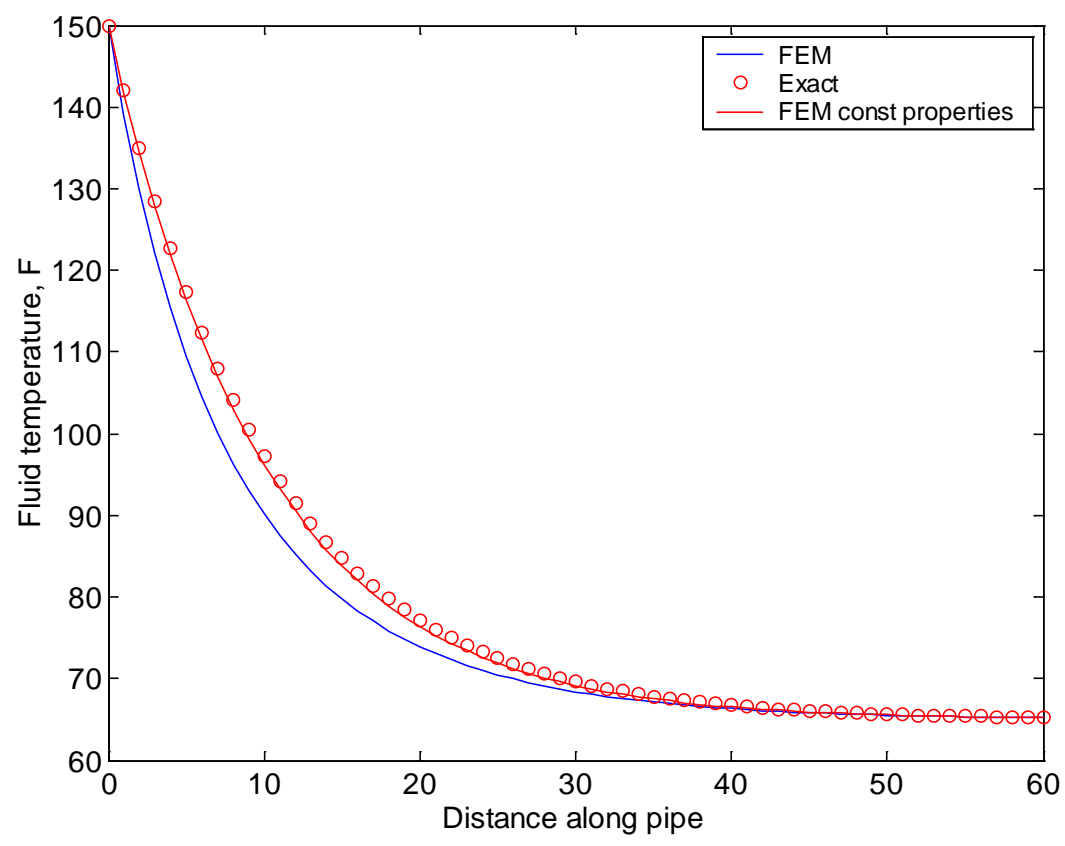

Fig. 2.7. Test case for integrated model (fluid and pipe). 
properties and wall temperature and given by Eq. (25). The second solid line in Fig. 2.7 corresponds to the FEM solution with constant properties, and it is seen to be very close to the exact solution. In fact, the RMS error between the exact and FEM with constant properties is only $0.57^{\circ} \mathrm{F}$.

\subsubsection{Free Convection from Cylinder}

In order to properly account for free convection from the pipe exterior, a suitable correlation must be used to determine $h_{o}$. A correlation suggested by Churchill and Chu, taken from Chap. 9 of Incropera and Dewitt ${ }^{1}$ will be used:

$$
\overline{N u}_{D}=\left\{0.60+\frac{0.387 R a_{D}^{1 / 6}}{\left[1+(0.559 / \mathrm{Pr})^{9 / 16}\right]^{8 / 27}}\right\}^{2}
$$

which is valid for $R a_{\mathrm{D}}<10^{12}$. For this, properties of air as a function of temperature are also needed. Data were taken in English units from Table B-2 of Hodge and Taylor ${ }^{2}$, and polynomial curve fits made using Excel. The relevant results:

$$
\begin{aligned}
& k=-4.96026 \mathrm{E}-09 T^{2}+2.14617 \mathrm{E}-05 T+1.33071 \mathrm{E}-02 \\
& v=4.24793 \mathrm{E}-10 T^{2}+4.59699 \mathrm{E}-07 T+1.29913 \mathrm{E}-04 \\
& \operatorname{Pr}=1.23708 \mathrm{E}-15 T^{6}-1.18411 \mathrm{E}-12 T^{5}+4.51551 \mathrm{E}-10 T^{4}-8.92890 \mathrm{E}-08 T^{3} \\
& \quad+9.58726 \mathrm{E}-06 T^{2}-5.13999 \mathrm{E}-04 T+7.30215 \mathrm{E}-01 \\
& g \beta / v^{2}=-1.77975 \mathrm{E}-01 T^{3}+1.30881 \mathrm{E} 02 T^{2}-3.57424 \mathrm{E} 04 T+4.19174 \mathrm{E} 06
\end{aligned}
$$

This last parameter is convenient for computing the $R a_{\mathrm{D}}$ for free convection. The largest error in these curve fit representations of the data is in the last parameter, and over the range of $0^{\circ} \mathrm{F}$ to $300^{\circ} \mathrm{F}$ the data are represented within $+0.66 \%$ or $-0.55 \%$.

\subsubsection{Forced Convection from Cylinder}

In the case of a known velocity normal to and over the outside of the piping, the heat transfer coefficient can be determined from the correlation of Churchill and Bernstein, taken from Incropera and Dewitt: ${ }^{1}$

$$
\overline{N u}_{D}=\left\{0.60+\frac{0.387 R a_{D}^{1 / 6}}{\left[1+(0.559 / \mathrm{Pr})^{9 / 16}\right]^{8 / 27}}\right\}^{2}
$$

For application of Eq. (27), $\operatorname{Re}_{D} \operatorname{Pr}>0.2$, and all properties are evaluated at the film temperature.

\subsubsection{Radiation from Cylinder}

Heat loss from the outside of the pipe by radiation is accounted for by the radiation heat transfer coefficient. This assumes that the temperature for external radiation exchange is the same as that of the environment for convection. The radiation heat transfer coefficient is given by:

$$
h_{\text {rad }}=\varepsilon \sigma\left(T_{\text {sur }}^{2}+T_{\infty}^{2}\right)\left(T_{\text {sur }}+T_{\infty}\right)
$$


For the steady solution for temperature with $150^{\circ} \mathrm{F}$ water entering a $0.5^{\prime \prime}$ diameter (Type M) copper pipe at 2.25 GPM, the $h_{\text {rad }}$ and the external convection coefficient are the same order of magnitude. The variation in heat transfer coefficient along the pipe is seen in Fig. 2.8 below.

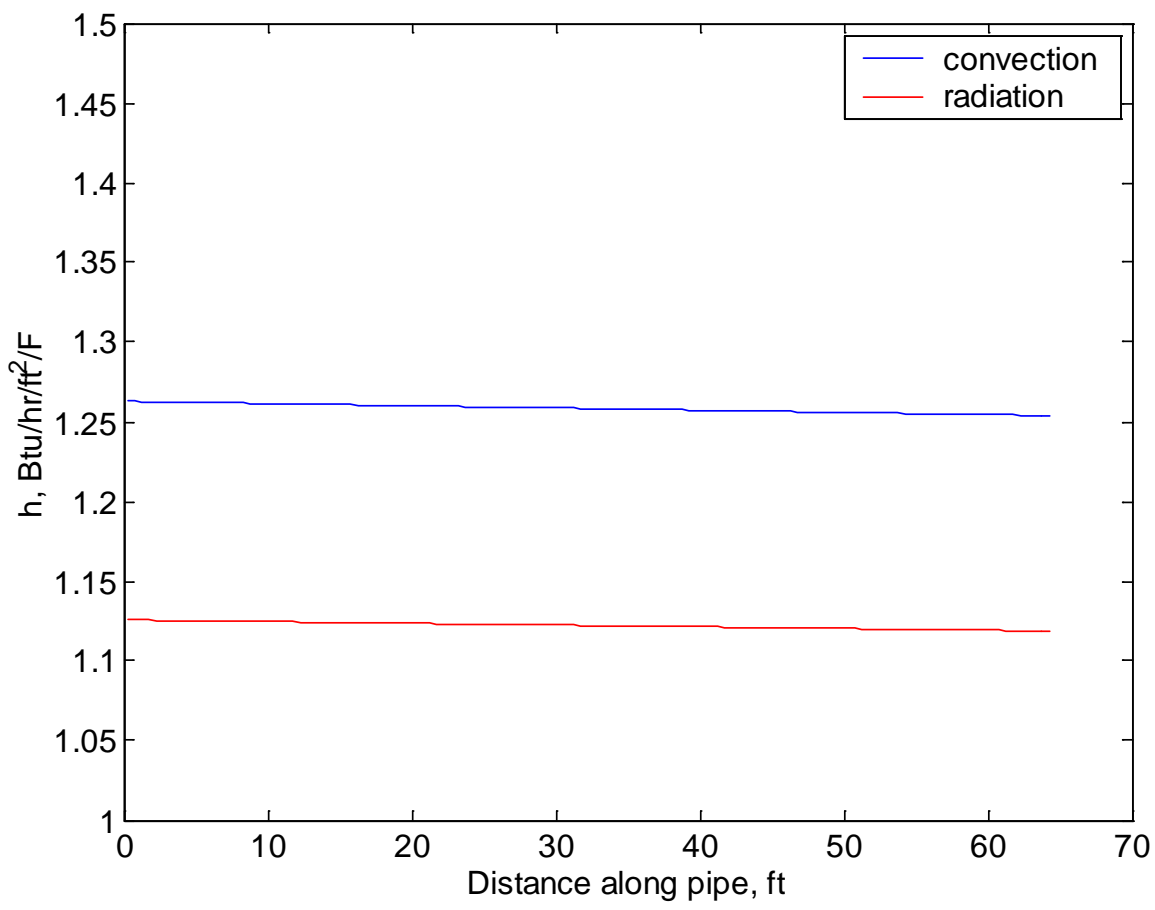

Fig. 2.8. Convection and radiation exchange coefficients on pipe exterior.

\subsubsection{Pure conduction in pipe}

When there is no flow in the pipe, we need a "heat transfer coefficient" to account for the heat conduction from the fluid to the pipe wall. To develop this, we take the correlation for heat conduction in a solid cylinder subjected to a step increase in temperature at its surface. This one dimensional transient solution can be found, for example, in Beck, et al.: ${ }^{5}$

$$
T(r, t)=T_{0}-T_{0} \sum_{n=1}^{\infty} \exp \left(-F o \beta_{n}^{2}\right) \frac{J_{0}\left(\beta_{n} r / b\right)}{\beta_{n} J_{1}\left(\beta_{n}\right)}
$$

where $F_{O}=\alpha t / b^{2}$, and $b$ is the radius of the cylinder. In Eq. (29), the $\beta_{n}$ 's are the roots of $J_{0}\left(\beta_{n}\right)=0$. We can use Eq. (29) to find the average temperature in the cylinder at any time and the heat flux from the cylinder at any time as:

$$
\begin{gathered}
T_{a v g}(t)=\frac{\int_{0}^{b} T(r, t) 2 \pi r d r}{\pi b^{2}} \\
=-T_{0} \sum_{n=1}^{\infty}\left(\beta_{n}^{2}+2 \exp \left(-\beta_{n}^{2} F o\right)\right) / \beta_{n}^{2}
\end{gathered}
$$




$$
\begin{aligned}
& q^{\prime \prime}=-\left.k \frac{d T(r, t)}{d r}\right|_{r=b} \\
& =\sum_{n=1}^{\infty} \frac{k T_{0}}{\exp \left(\beta_{n}^{2} F o\right) b}
\end{aligned}
$$

We define the heat transfer coefficient for this situation as

$$
h=\frac{q^{\prime \prime}}{\left(T_{0}-T_{a v g}\right)}
$$

and this can be put in a dimensionless form in terms of $\mathrm{Nu}_{D}=h D / k$. If the $\mathrm{Nu}_{D}$ is plotted versus the dimensionless temperature difference $\Delta T=\left(T_{0}-T_{\text {avg }}\right) / T_{0}$ for a range of $k, F o$, and $b$, then a unique curve results (see Fig. 2.9). A simple polynomial curve fit can represent the data in the figure:

$$
\begin{aligned}
\mathrm{Nu}_{D} & =2.9892 \mathrm{E}+06-4.6524 \mathrm{E}+06(\Delta T)+2.9096 \mathrm{E}+06(\Delta T)^{2}-0.93157 \mathrm{E}+06(\Delta T)^{3} \\
& +1.6132 \mathrm{E}+05(\Delta T)^{4}-1.4528 \mathrm{E}+04(\Delta T)^{5}+5.9960 \mathrm{E}+02(\Delta T)^{6}-7.9867 \mathrm{E}+00(\Delta T)^{7} \\
& +5.7870 \mathrm{E}+00(\Delta T)^{8}
\end{aligned}
$$

The points computed using the curve fit are the circles in Fig. 2.9.

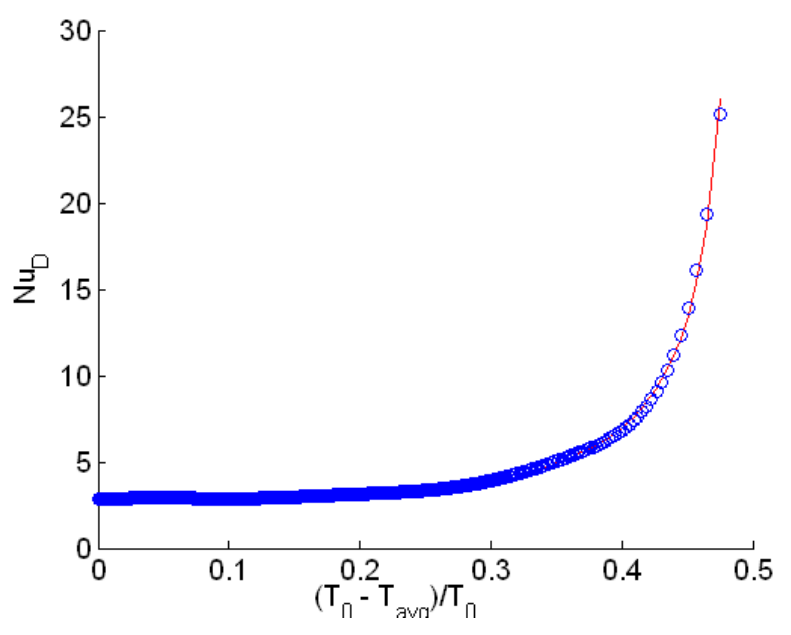

Fig. 2.9. $\mathrm{Nu}_{D}$ for conduction from analytical solution.

\subsubsection{Soil/Attic model}

If a pipe is surrounded by a relatively large layer of attic insulation or soil or concrete slab, we take account of this by including in the model a finite radial thickness of the external material. This is basically the same as if the pipe (with or without pipe insulation) is further "insulated" with a thickness of attic insulation or soil or concrete.

The situation is depicted in Fig. 2.10. The layer of surrounding material is characterized by a thickness parameter, thick, and this thickness of material is assumed to be all around (360 degrees) the pipe. The outer surface of this resulting composite cylinder is assumed to be subjected to a convective/radiative boundary. However, it is tacitly assumed that the simulation time is relatively short, and that the temperature on the outside of this large cylinder of additional material will not 
change substantially during the calculation. Therefore, it will not matter that one surface of the material is exposed to convection and the others are semi-infinite (as in the case of a buried pipe) or if one surface has convection/radiation to a lower temperature than the other (as in the case of attic insulation). If simulation run times are extremely long (hours), then this simple model of the attic/soil will be invalid. However, the hot water supply in most residential hot water heaters would typically be exhausted for such long run times.

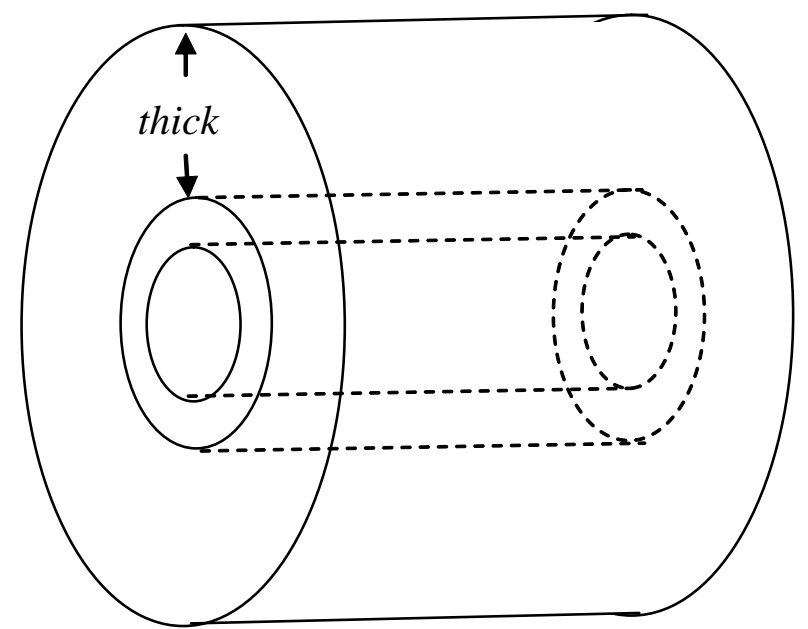

Fig. 2.10. SOIL/ATTIC material of thickness thick surrounding pipe/insulation. 


\section{USER GUIDANCE}

The hot water distribution calculation package consists of two main components: the core calculation program (described in Sect. 2) and the user interface program. The latter was produced by Dr. Roberto Lenarduzzi of Oak Ridge National Laboratory using LabVIEW. The core program reads text files as input and produces text files as output. It performs a single calculation each time it is run, where that calculation can consist of a combination of pipe segments over a specified length of time. The interface program allows the user to provide input and read output using Excel files, and assists the user by running the core program multiple times and combining output into a form more readily usable. The program has been run successfully under both the Windows 2000 and Windows XP operating systems.

This document describes the operation of the user interface. In this guide it is assumed that the application and associated files reside in a directory structure as defined in Appendix A.

\section{Installation of LabVIEW Engine}

Prior to running the user interface, the LabVIEW run time engine needs to be installed. This is done using the file "LVRunTimeEng.exe." Execute this file on the computer to be used for the calculations, and accept all the defaults.

\section{Data Input}

A simulation requires the preparation of input data files prior to starting the application. These files are as follows:

1. The pipe property file is an Excel file containing information for various types of pipes (precise dimensions, heat transfer properties, etc.). The file must be called pipe.xls and be in the "properties" sub-directory. The pipes used in the simulation are selected from this file. This file is described in detail in Appendix B. It is important that the entries for material, diameter and type (the entries in the first 3 columns of pipe.xls) match exactly with the specifications for those parameters contained in the input data file. The program looks at these entries in the input file and then tries to find a matching set in pipe.xls. When a match is found, the program uses the detailed pipe information for its calculation. Pipe.xls can contain information on as many types of pipe as desired and needs to be edited only if there is a need to add the property definitions of new types of pipe.

2. The insulation property file is an Excel file containing information for various types of insulation (heat transfer properties) and for materials that may surround the pipe, such as clay or sand. The file must be called insulation.xls and be in the "properties" sub-directory. Both the insulation and the material surrounding the pipe used in the simulation are selected from this file. This file is described in detail in Appendix C. It is important that the entries for material (the entries in the first column of insulation.xls) match exactly with the entries for "Pipe Insul" (the insulation material surrounding the pipe) or "Surr Insul" (the material around the pipe and insulation, such as soil) contained in the input data file. The program looks at these entries in the input file and then tries to find a matching entry in insulation.xls.

When a match is found, the program uses those heat transfer properties in its calculation. The second column in the insulation file must have the word, "any". This is an artifact of an earlier version of the program in which the second column was used as additional identification for the insulation. While the second column is no longer used, the input portion 
of the interface program still looks for this entry in this column. Insulation.xls can contain information on as many types of insulation or material as desired and needs to be edited only if there is a need to add the property definitions of new types of insulation or material.

3. The input data file is an Excel file that provides basic data about the configuration to be analyzed. The filename is chosen by the user; this name will be used as part of the name of the output file when this file is created by the program as it runs. A sub-directory will be created under the "OutputData" directory that has the same name as the input filename; inside this directory will be an Excel file created by the program that uses the name of the input file with “_results” appended to it. The input data file describes a collection of possible locations for hot water draws. Each location is represented by a line in this file. The usage file dictates in what sequence draws occur and the time intervals between draws. As guided by the usage file, the user interface will call the core program to calculate an individual simulation run for each line. Each line of the input file labels the draw location, provides its flow rate, and describes the piping configuration through which the flow occurs. This configuration can consist of multiple segments. This file is described in detail in Appendix D. It is assumed-in this implementation of the application - that the pipe material and pipe type (e.g., copper, type $\mathrm{K}$ ) is the same for all segments, although the diameters of each segment can be different. It is also assumed that the insulation material, if present is the same for all segments. The thickness of the pipe insulation can vary from segment to segment, and zero thickness can be used to indicate an absence of insulation, but the program cannot, at present, accommodate a change from one type of insulation to another within the same line of the input file. The type of material surrounding the pipe and its insulation can vary from one segment to another. It is also assumed that no other events are occurring at the same time so that the flow into the first segment is the same as that out of the last segment. Note that since the flow rate for a given location is specified on the input file entry, if one wants to calculate a second draw at the same location, but with a different flow rate, a second line in the input file will be needed (and so on for additional draws at the same location if the flow rates vary). The same line in the input file may be called more than once in the usage file. However, if the user wants to keep the intermediate text files used by the program, each subsequent call of the same line number will overwrite the text files from the earlier call. The Excel output file will not be affected by this, but it is relevant if the user wishes to examine or retain the intermediate text files. In such a case, a separate line in the input file should be provided for each draw that occurs, avoiding calling the same input line more than once.

4. The usage data file is an Excel file that prescribes the sequence of flows (defined in the Input Data File) to be simulated. This sequence is determined by the number in the "Flow Usage" column (column A); each number is compared to the entries in the "Event ID" column of the input file, and the appropriate line of information taken from the input file for the calculation. The text in the "Outlet" column is purely for user convenience. The usage file may have any name chosen by the user. It also defines the cooling period (if any) between flows and whether the simulation should use the ambient temperature or the average temperature of the water after the cooling period as the start water temperature for each segment. This file is described in Appendix E and must be stored in the "UsageData" directory. The user interface calls the core program at least once for each line in the usage file. If the entry in the "Time Before Outlet Use” column (column C) is greater than zero, then the interface program will call the calculational program to perform a zero-flow, cooldown calculation for the cited line. The cooldown calculation assumes that the water in each segment is at the temperature at the end of the previous calculation. The results of the cooldown calculation are then available as input to the normal calculation done for the line in question. The usage file has a column for each segment of a given draw location labeled “Temp Use.” If the entry in this column is 
Tamb, then the program assumes that the starting temperature for the water in this segment is the ambient temperature specified in the input file. However, if a line in the usage file includes the specification Tnew for a segment, it uses as a starting temperature the results of the cooldown calculation for that segment. Note that when performing a cooldown calculation, the program assumes that the results of the draw immediately preceding the current draw is the starting point for the cooldown. This must be kept in mind when using the Tnew option. For example, suppose one models a typical trunk and branch system with the length of trunk between the hot water heater and each branch as segment 1 , and the length of each branch as segment 2. Suppose there are 2 possible draw locations, A and B. Suppose the usage file calls for two successive draws at either location. For the second draw at the same location, entering the interval between the draws in the third column of the usage file and Tnew for both segments in the Temp Use column will cause a correct calculation for the cooldown between the draws and thus provide accurate information for the second draw. However, if the usage file called for a draw at A, then a draw at B, and then another draw at A, if Tnew is used for the branch segment of the second draw at A, an erroneous answer will result - the program will have assumed that the second segment for the second draw at location $\mathrm{A}$ is the same as the second segment for the previous draw, but the previous draw is at location B, which is NOT the same branch segment. So, at present, the Tnew choice for Temp Use will only provide correct results when the same location is called by the usage file multiple times in a row. It would be possible to modify the program to keep track of segment temperatures separately for individual draw locations, but constraints on resources available for development of the program have precluded doing that as of this date.

\section{Running the Application}

Once the input data files are prepared, running the application is just a series of small steps.

When the application is started, by double clicking the executable file, HWD_v42.exe, the main panel (Fig. 3.1) is shown to the user. If this panel does not fit appropriately on the monitor, changing the screen resolution may be necessary (through the Settings tab on the Display dialog box, reached by double clicking on "Display" in the Control Panel). A resolution of 1024 by 768 pixels or higher should produce an appropriate image.

The diagram shown by the program as background for this window is only symbolic of the type of calculation being performed; the program does not draw a map of a system provided by the user. Presently the difference between the "Conventional" and "Parallel Pipe" configuration selections is strictly cosmetic. Although a different background picture is provided, the functioning of the program is identical in both cases. The differences in the layout of one type of system compared to the other is embodied in the different data provided in the input Excel file. In contrast, the "Demand Recirculation" and "Total Recirculation" configuration selections change the functioning of the program. As a result, user must first select the piping configuration (labeled "Step 1" on the screen).

For both the conventional and parallel piping the interface program calls the calculational program with the computations being guided by the input Excel file and the usage Excel files (which will be chosen from the next screen). For both types of recirculation calculations, the existence of a second input file is required. This second input file has the same name as the base input file, except "-main" is appended to the file name. (For example, if the base input file is "House 1.xls", the second input file must be "House 1-main.xls") The base input file contains a description of the branches connecting the draw points to the recirculation loop. The "-main" file contains a single line that describes the recirculation loop. For continuous recirculation systems, the program uses the "-main" 


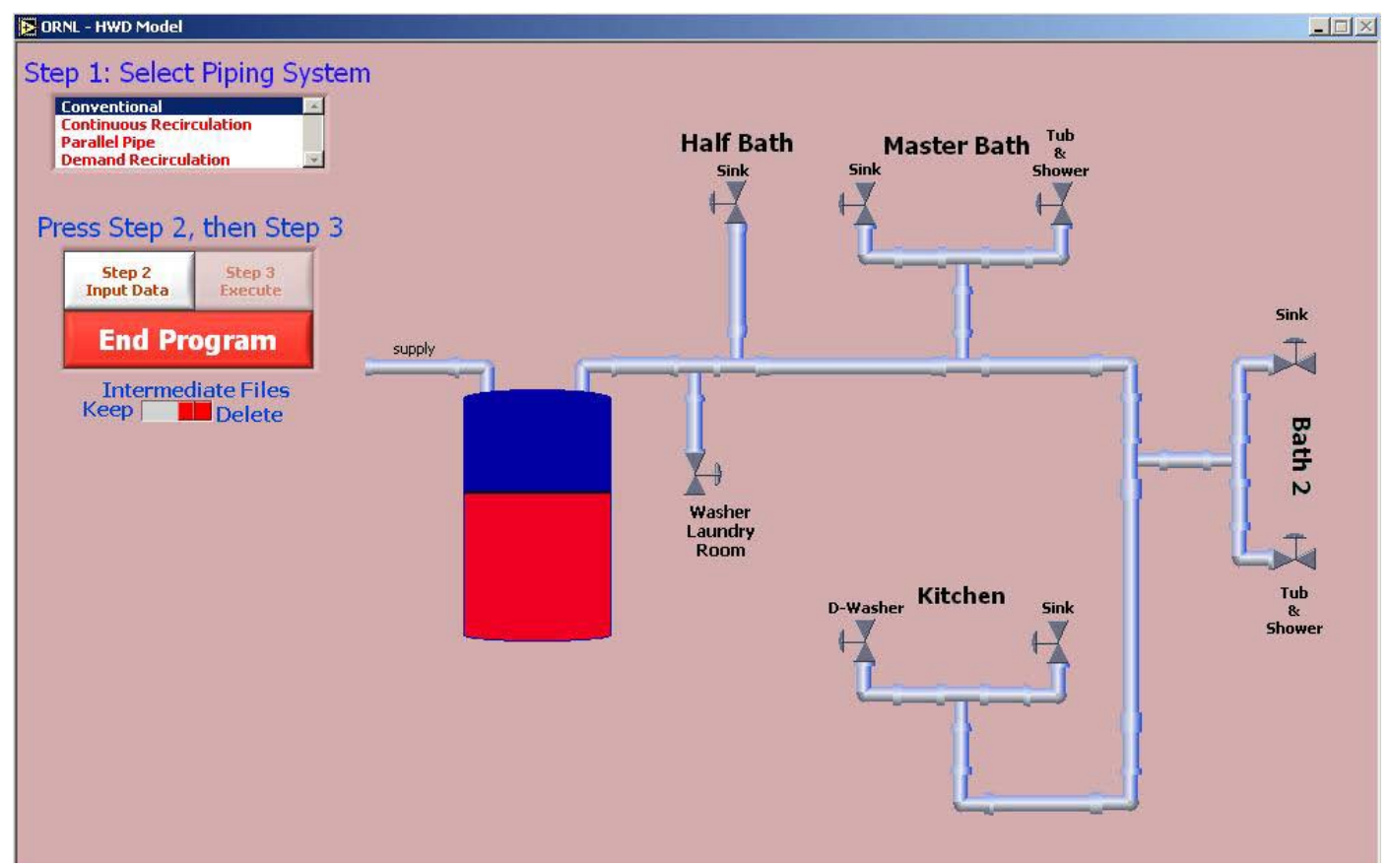

Fig. 3.1. ORNL-HWD Simulation main window.

file to compute the energy loss from the recirculation portion of the system over 24 hours, and reports this in the output Excel file. The usage file controls the calculations for the branches, just as for a conventional system. For demand recirculation systems, the program uses the “-main” file to calculate the time and energy consumed by the recirculation loop in reaching $105^{\circ} \mathrm{F}$ from a cold start (i.e., starting from the temperatures specified in the -main input file). These times and energy losses are then added to the times and energy losses computed for the branches (using the base input file), and the sums reported in the summary results in the Excel output file. The program does not, at present, include provisions to allow a reasonable calculation of the effects of clustered draws for demand recirculation systems. Therefore, for a demand recirculation case, the usage file should have all times between events set to 0 , and all temperatures set to Tamb.

As mentioned above, the user interface program calls the calculation program multiple times. The calculation program reads from and writes to text files. The interface program summarizes the results from these text files into an Excel file. If the user is only interested in the Excel output file, the default option of deleting the text files can be accepted. However, if the user wishes to keep the text files, the switch on this screen can be moved to the left (by clicking on the left portion of the slide) to accomplish this. A description of the intermediate text files is provided in Appendix F.

With the piping configuration selected, the Step 2 Input Data button is pressed to show the Simulation Data Input window as shown in Fig. 3.2. Data inputs are required for the green buttons on the menu.

(“Click to Select Simulation Input Data File”) opens a Windows Explorer window that has the contents (Excel files) of the InputData directory (Fig. 3.3). Normally, the user selects a file from this directory. Alternatively, the file can be selected from another directory by browsing within the Explorer window. For both recirculation systems (demand and total), two input files are used, but only one input file (the branches file without -main.xls) needs to be selected. The other vital input file In this window, the data input files are selected first. Clicking on illuminated, right green button 


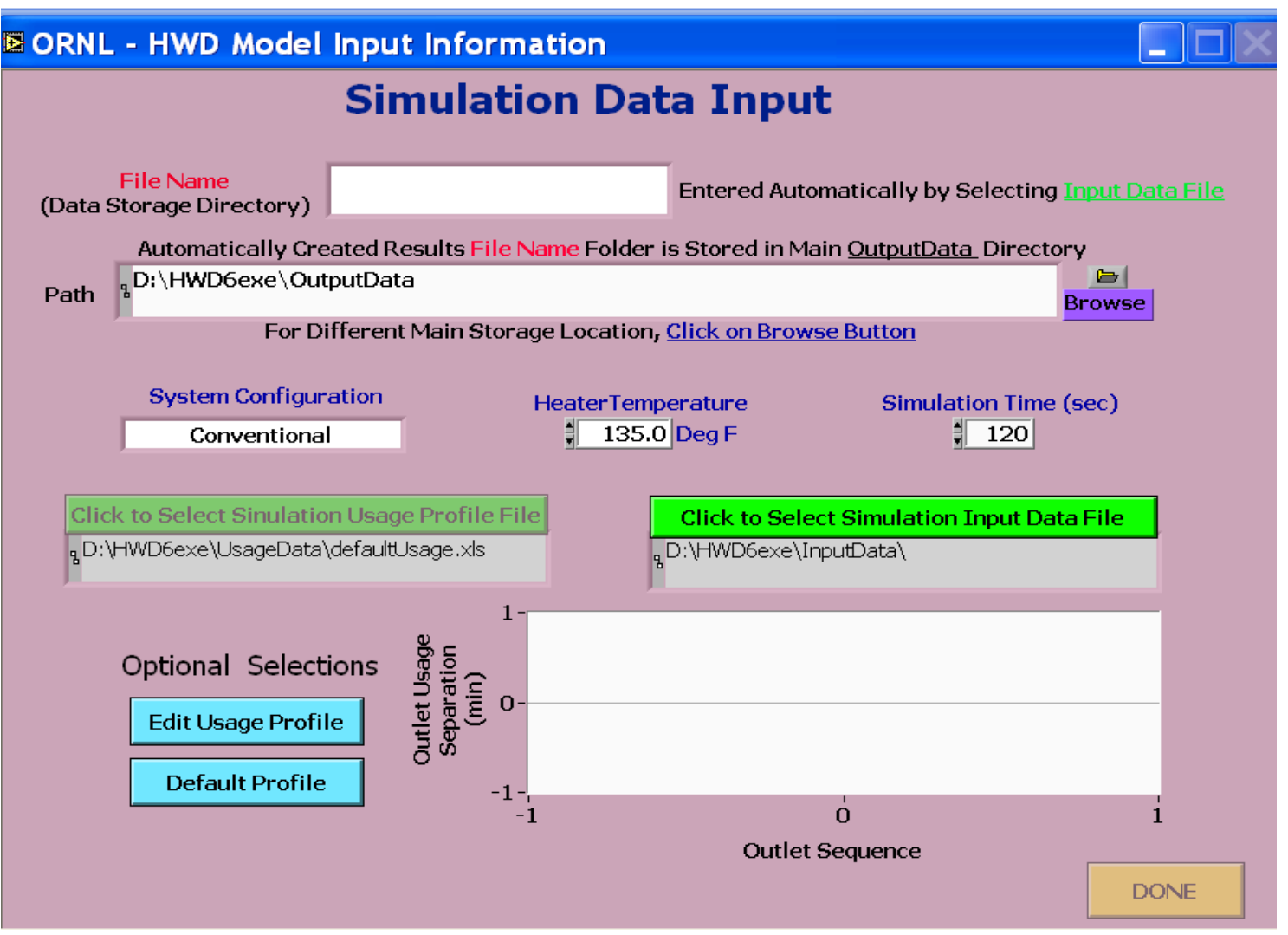

Fig. 3.2. Simulation input data window (Step 2) for all cases except demand recirculation.

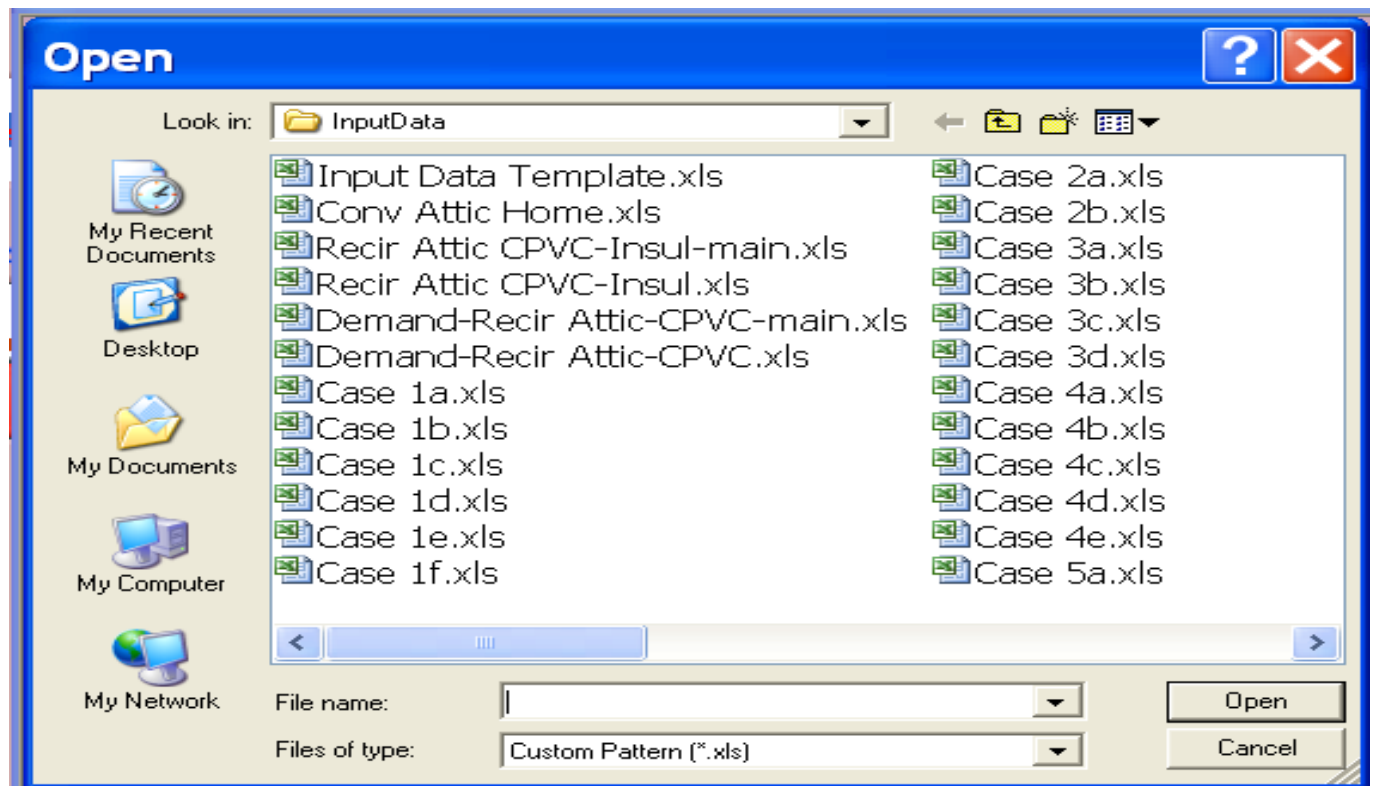

Fig. 3.3. Explorer window showing contents of InputData directory. 
(*-main.xls) will be selected automatically based on the user's piping system selection on the front menu. The information $\left(^{*}\right)$ prior to -main.xls must be identical to that of the branches input (*.xls) file.

Next, the usage file is selected in the same manner by clicking on the now illuminated, left green button ("Click to Select Simulation Usage Profile File”), which opens an Explorer window (Fig. 3.4) revealing the contents (Excel files) of the UsageData directory. For the demand and continuous recirculation systems, the usage file only affects the calculations performed for the input file which contains the branches. The user can navigate to another directory if desired.

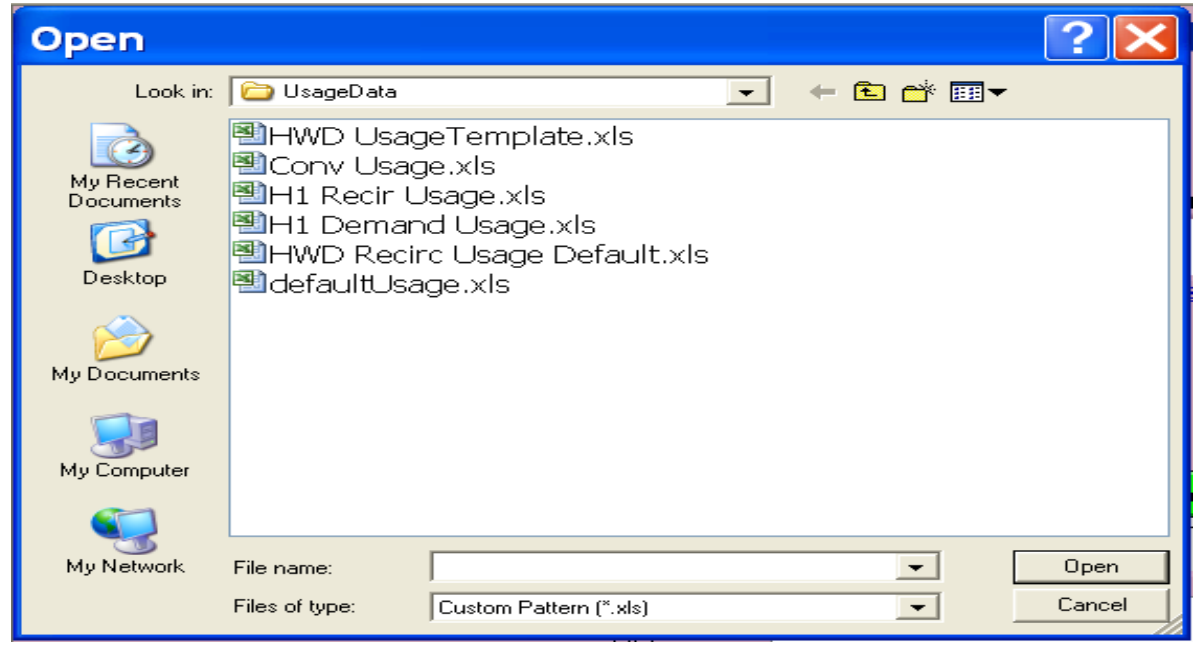

Fig. 3.4. Explorer window showing contents of UsageData directory.

If the user selected the demand recirculation pipe configuration, then the Simulation Data Input screen will have one additional feature (Fig. 3.5)

The additional feature is the "Demand Circulation Main Loop Simulation Time (sec)" value specified on the right side of the screen. The default value is 38 seconds, but this can be changed by the user. The typical installation of a demand recirculation system will be such that when a demand for hot water is received, the recirculation loop runs until a preset temperature (such as $105^{\circ} \mathrm{F}$ ) is reached, and then stops. However, the core calculational program currently has no provision to stop at a set temperature. In order to approximate this behavior, if the user has some idea of when the preset temperature is typically reached, this time can be set on this screen. One way to get an estimate of this time would be for the user to run a separate calculation, using the conventional configuration option and an input file that has a single line that describes the recirculation loop. The result of that calculation would provide the time to reach $105^{\circ} \mathrm{F}$, and that time could then be entered on this screen for the demand recirculation calculation.

After the file selections have been made, all the remaining fields within the simulation input windows are automatically filled (Fig. 3.6 ). Before clicking DONE, the user may click the "Edit Usage Profile" button an manually alter the usage profile provided by the selected usage file, or the user may click the "Default Profile" button, which causes the program to use as the usage file a file named defaultUsage.xls, which the interface creates, and which sets up all draws as cold draws (i.e., all time intervals are set to zero, Tamb is specified for all segments, and the order of the calls matches the order in which the lines appear in the input Excel file. 


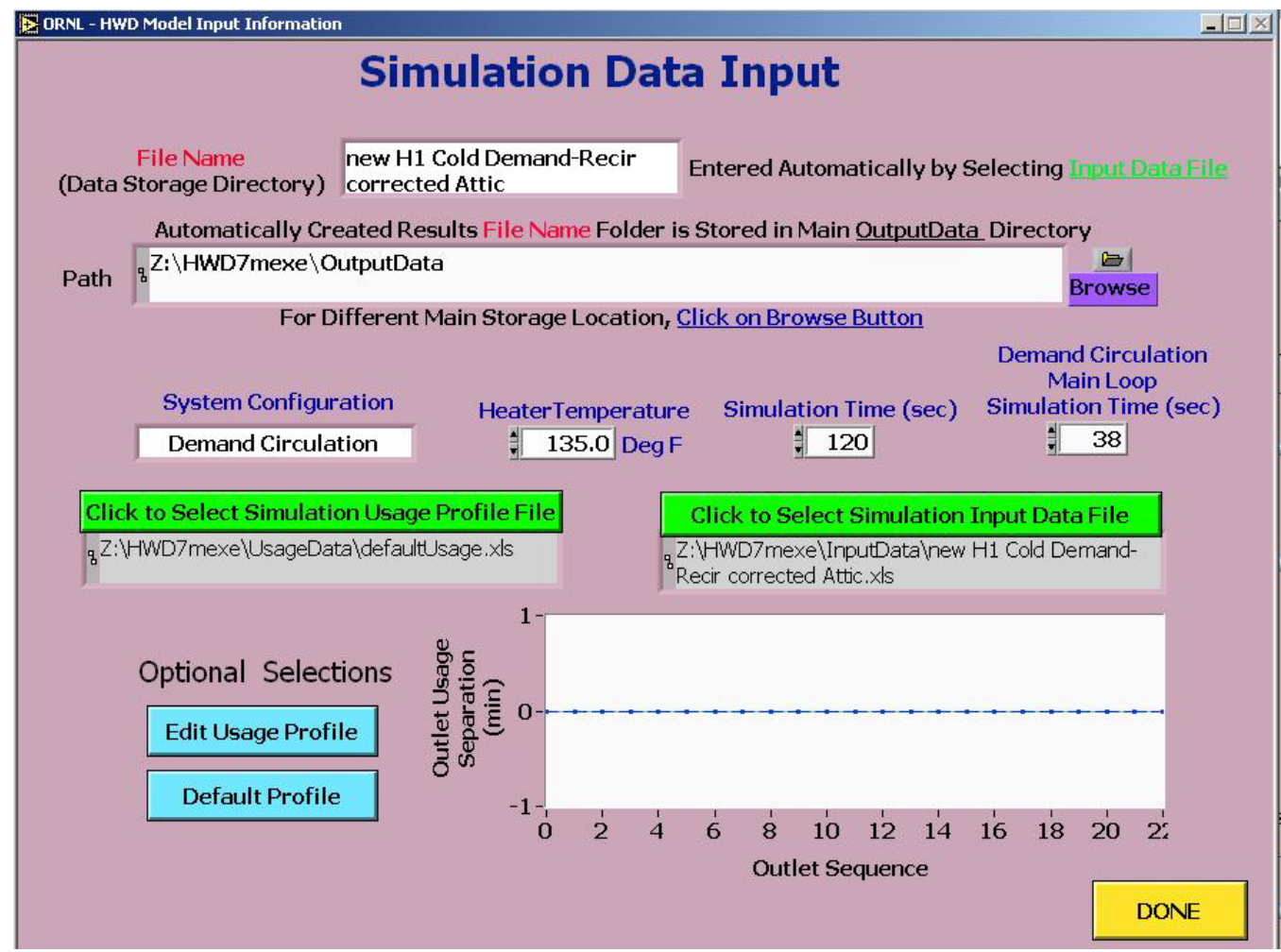

Fig. 3.5. Simulation input data window (Step 2) for demand recirculation case.

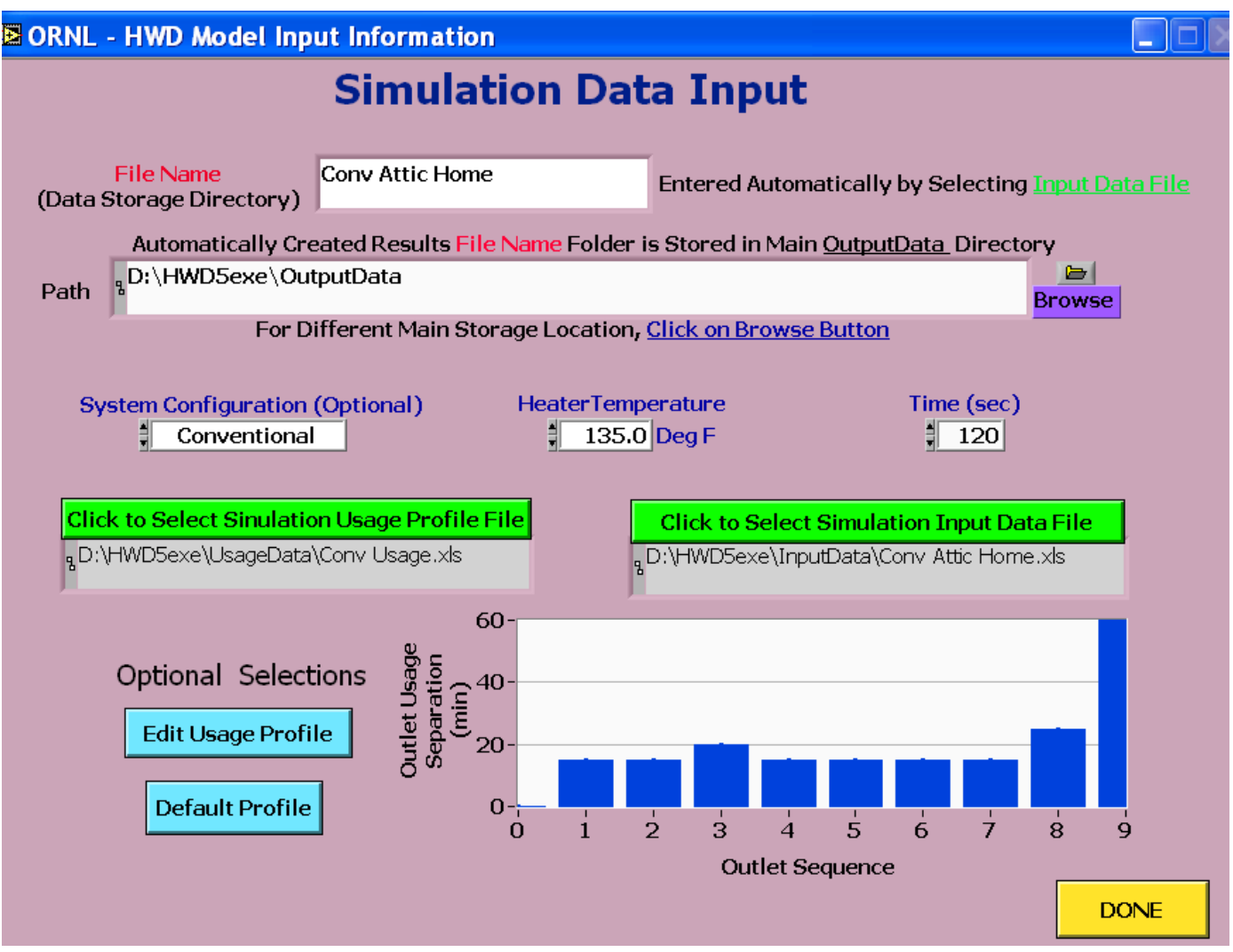

Fig. 3.6. Simulation Input Data window after selections completion. 
After the DONE button is pressed, the model returns to the main menu where the Step 3 Execute button is now lighted, and it should now be pressed to start the simulation execution (see Fig. 3.7).

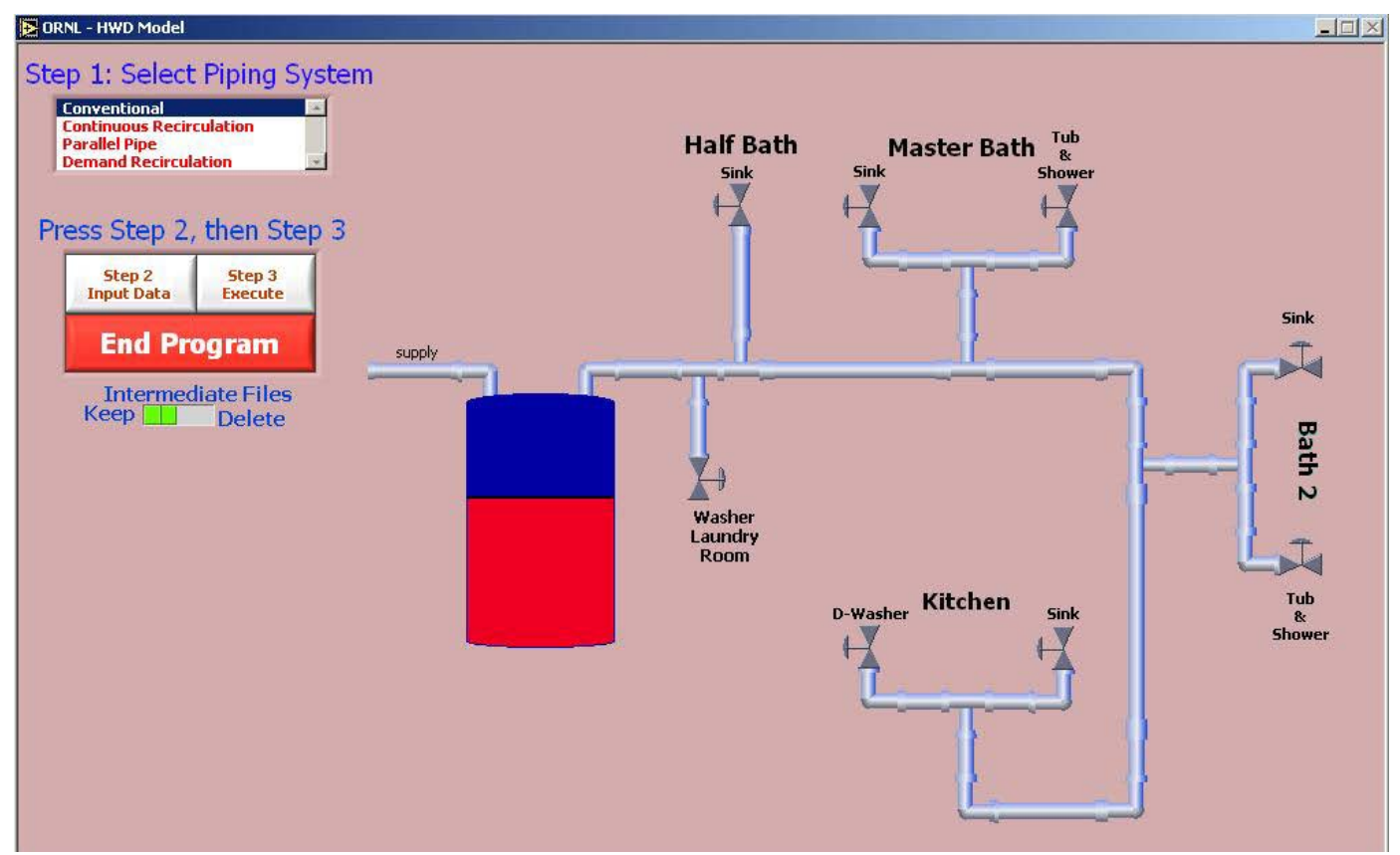

Fig. 3.7. ORNL-HWD Simulation main window after input data selection is completed.

Once the "Step 3 Execute" button is clicked, the progress is noted in a light pink box located on the lower left of the screen, and it can be stopped by pressing the red, "Abort Simulation” button located on the bottom left of the menu, as seen in Fig. 3.8.

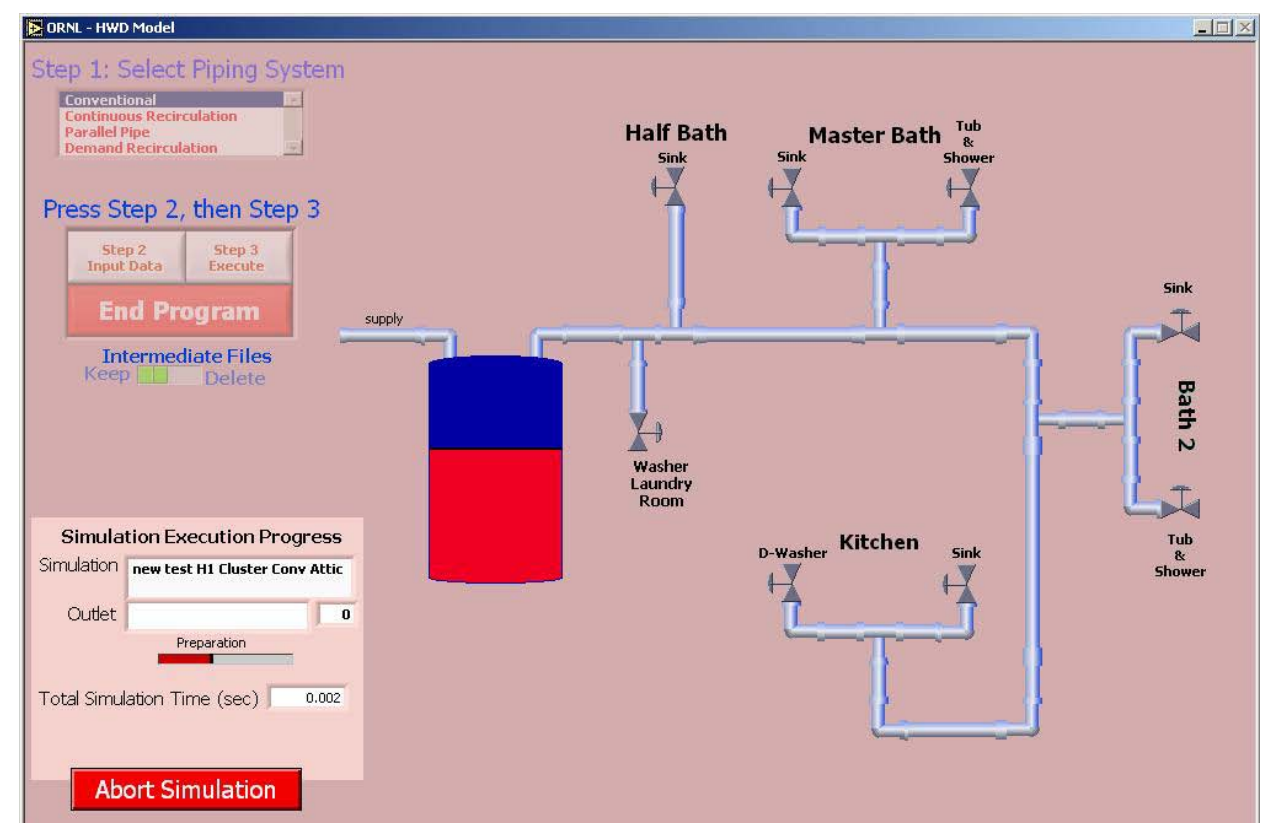

Fig. 3.8. ORNL-HWD Simulation main window while computations are in progress. 
After the computation, the results file is stored in the main OutputData directory that can be accessed directly from the ORNL-HWD model completion menu (yellow, "View Output" button, Fig. 3.9). Another calculation can be executed by pressing "Run New Simulation" button or terminated by press the "End Program” button.

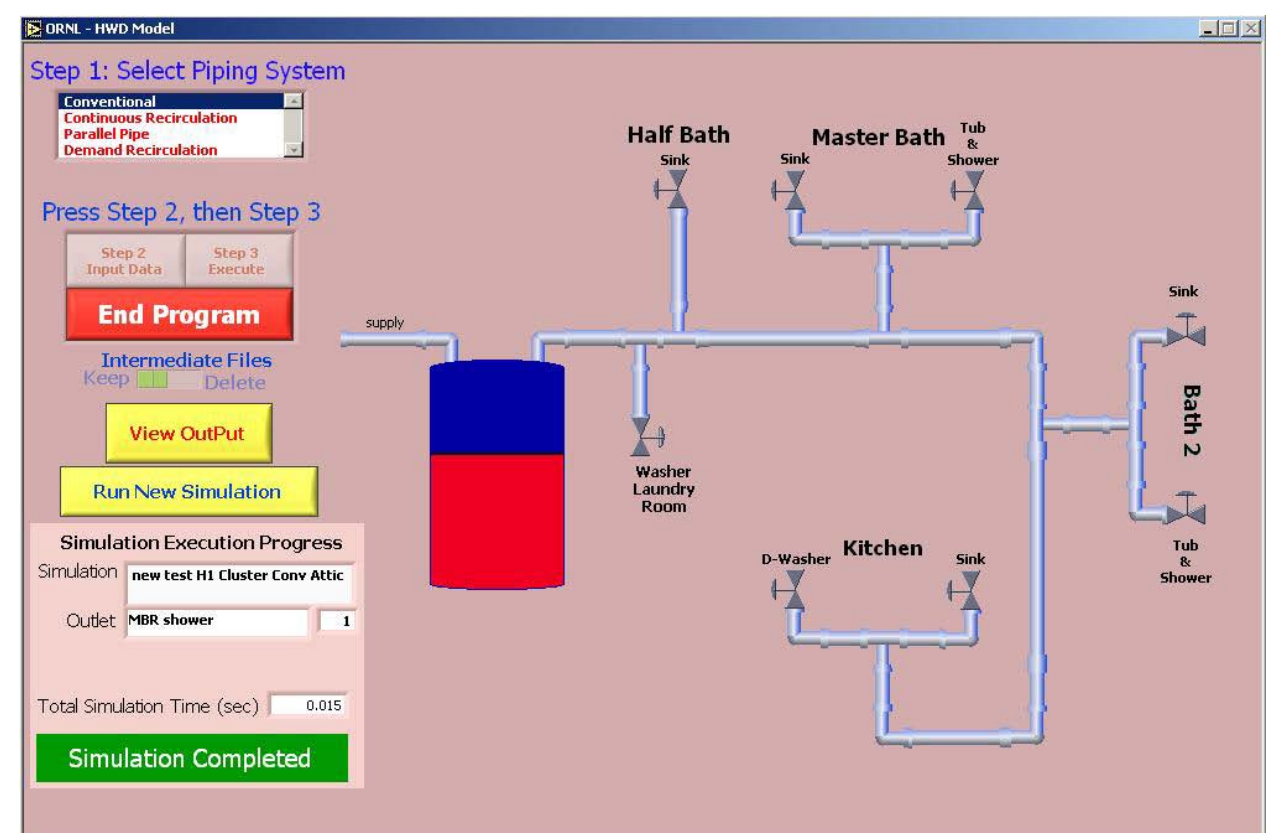

Fig. 3.9. ORNL-HWD Simulation main window when computation is completed.

\section{Program Output}

The program summarizes the output from the calculation in a subdirectory created under the OutputData directory. The subdirectory carries the name of the input file. Within this subdirectory is an Excel output file with the same name as the input file but with "-results" appended to the name.

If the user selects the "keep” option under "intermediate files" on the first screen drawn by the interface program, the user interface generates copies the input and output text files for each use of the calculational program to this same subdirectory. In order to distinguish the text input and output files from one another, and to keep them from being overwritten, the naming convention used is "in_xx.txt" and "out_xx.txt" where $x x$ is a two digit representation of the event number (taken from the entry for each event in the first column of the input Excel file).

If the usage file specifies that the "Time Before Before Outlet Use" is greater than 0, the user interface will also cause a cooldown calculation to be performed before the event calculation itself is performed. This cooldown calculation uses zero flow, and takes as its initial conditions the ending conditions of the previous calculation. The input and output text files for cooldown calculations have the format "in_xxp.txt" and "out_xxp.txt" and are also saved in this directory. Details of the text file formats is provided in Appendix F.

The output Excel file will contain 3 worksheets for conventional or parallel pipe configurations, and 4 worksheets for demand recirculation or continuous recirculation configurations. The first 3 worksheets for all files have a similar configuration. The first worksheet summarizes the results of the calculation (Fig. 3.10). 


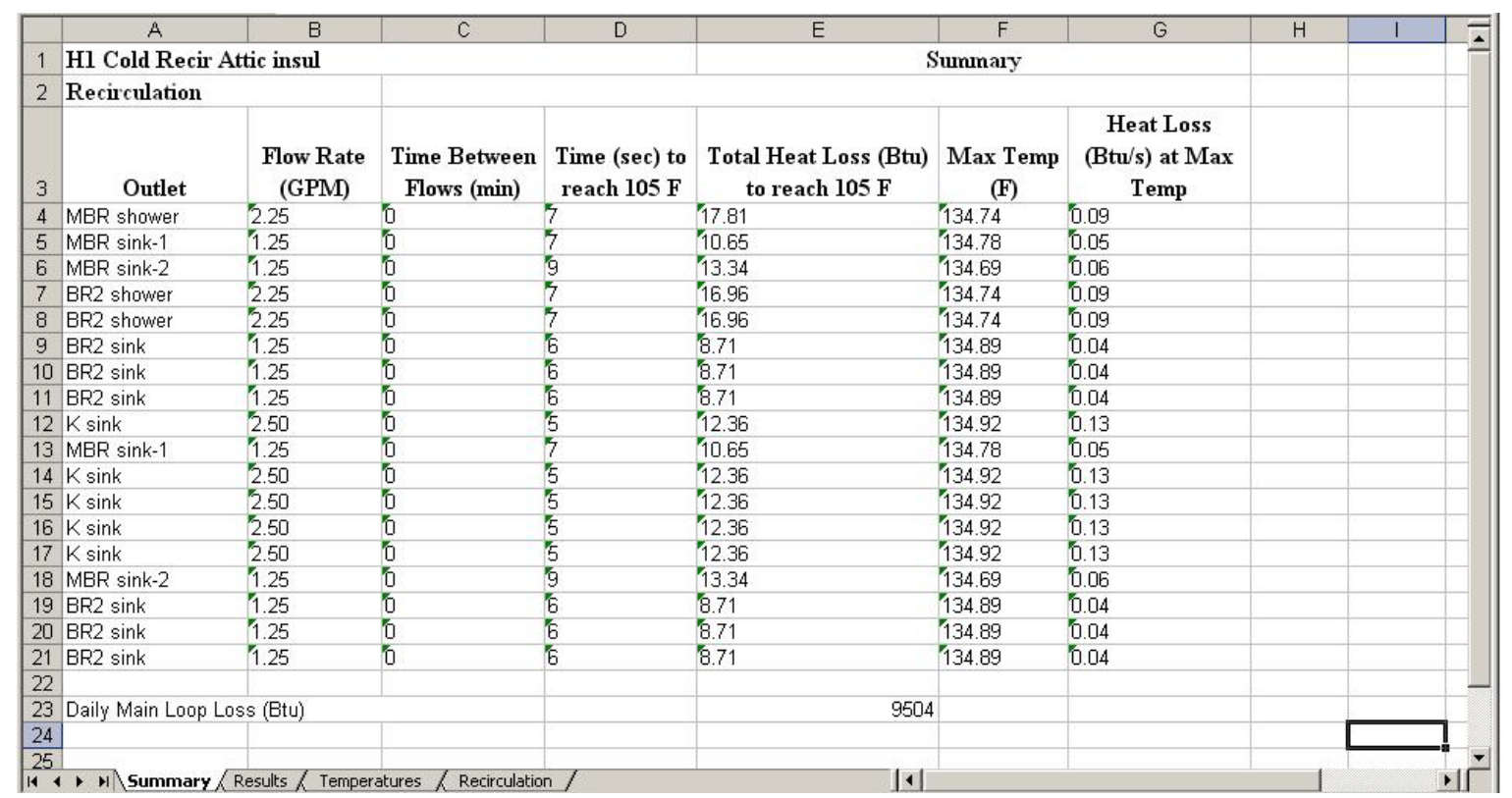

Fig. 3.10. Summary worksheet from results file for continuous recirculation case.

As can be seen in Fig. 3.10, the continuous recirculation configuration includes a value for the heat loss in Btu from the recirculation loop at the bottom of this worksheet. This line is absent from the other configurations.

The second worksheet is labeled "Results," and contains more detailed information on the results of each computation for each segment of each event (Fig. 3.11).

Some of this information is self-explanatory. TWOut provides the outlet temperature from the segment (called sections on this page). Qloss provides the heat loss rate computed based on an energy balance. The heat loss rate is also calculated based on the convective heat transfer from the fluid to the pipe wall. Although these values are not included in the Excel file for each time step (they are provided in the intermediate text output files), the total heat loss for the entire calculation is provided by both methods (lines 20 and 21 in Fig. 3.11). hd and ho are the heat transfer coefficients on the inside and outside of the pipe, respectively. $\mathrm{UA} / \mathrm{L}$ is the overall heat transfer coefficient per unit length. If the gap model is NOT used, the calculational program does not compute this quantity, and all the values under this heading will be zero. If the gap model IS used, this value is computed as reciprocal of the sum of thermal resistances of the inside heat transfer coefficient, the pipe wall, the insulation (if present), and the external heat transfer coefficient and radiation.

The third worksheet is labeled "Temperatures" and provides detailed temperature information from the calculation for each segment of each event at each time step (Fig. 3.12).

The continuous and demand recirculation cases include a 4th worksheet giving information on the calculation for the recirculation portion of the system (as described in the "-main" input file). This 4th worksheet is called "Demand" for the demand recirculation cases and "Recirculation" for the continuous recirculation cases. 


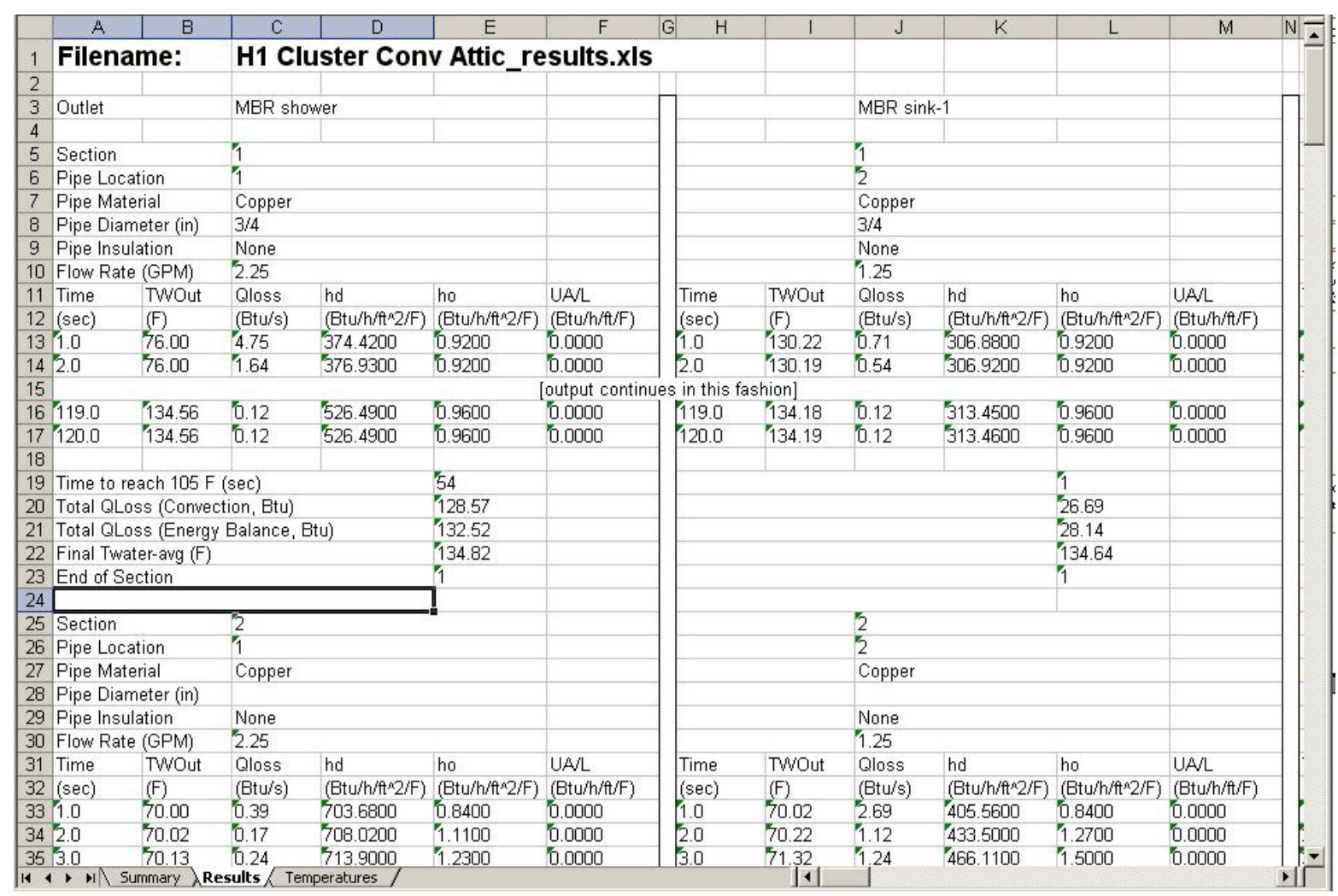

Fig. 3.11. Results worksheet from results file for conventional case.

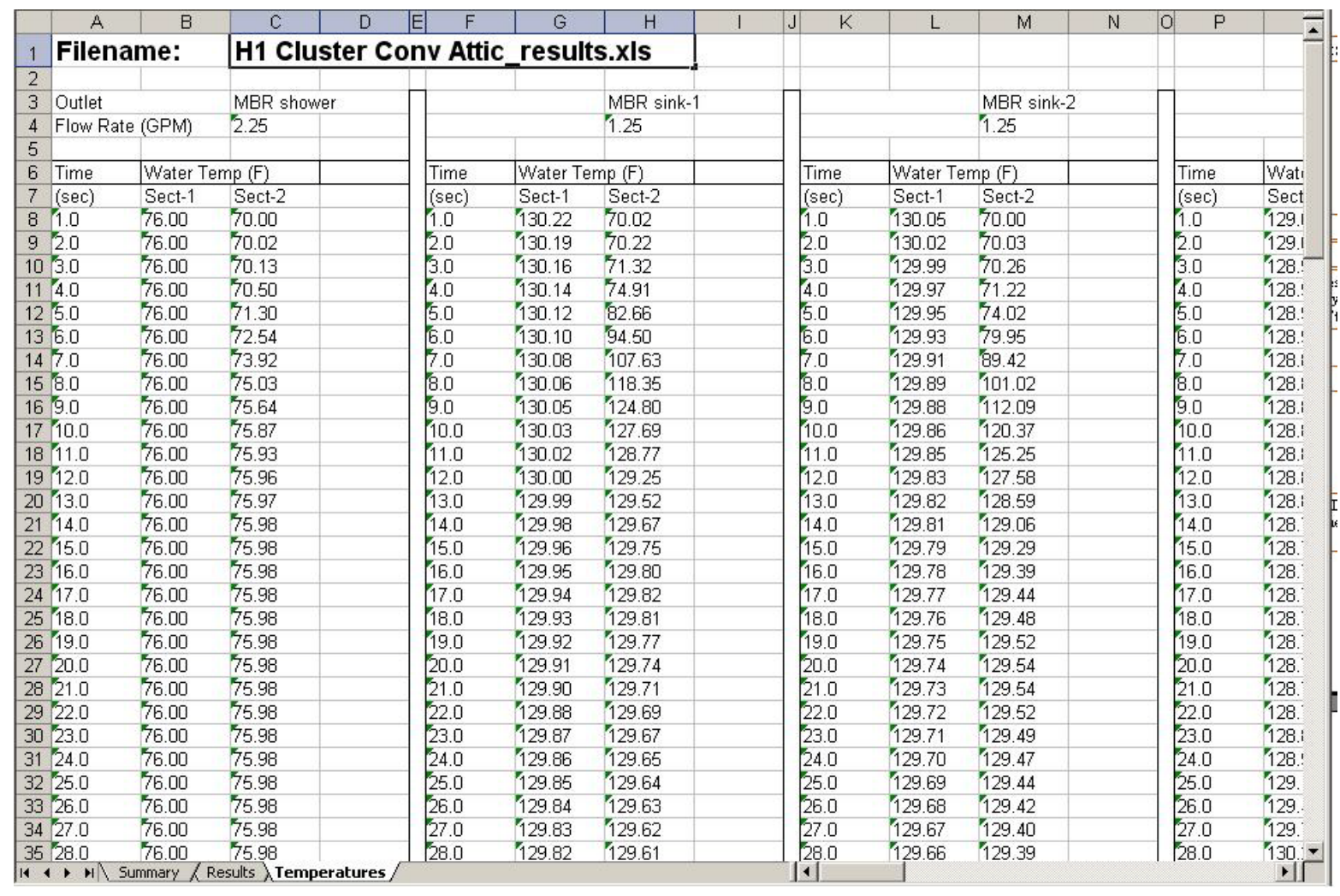

Fig. 3.12. Temperature worksheet from results file for conventional case. 


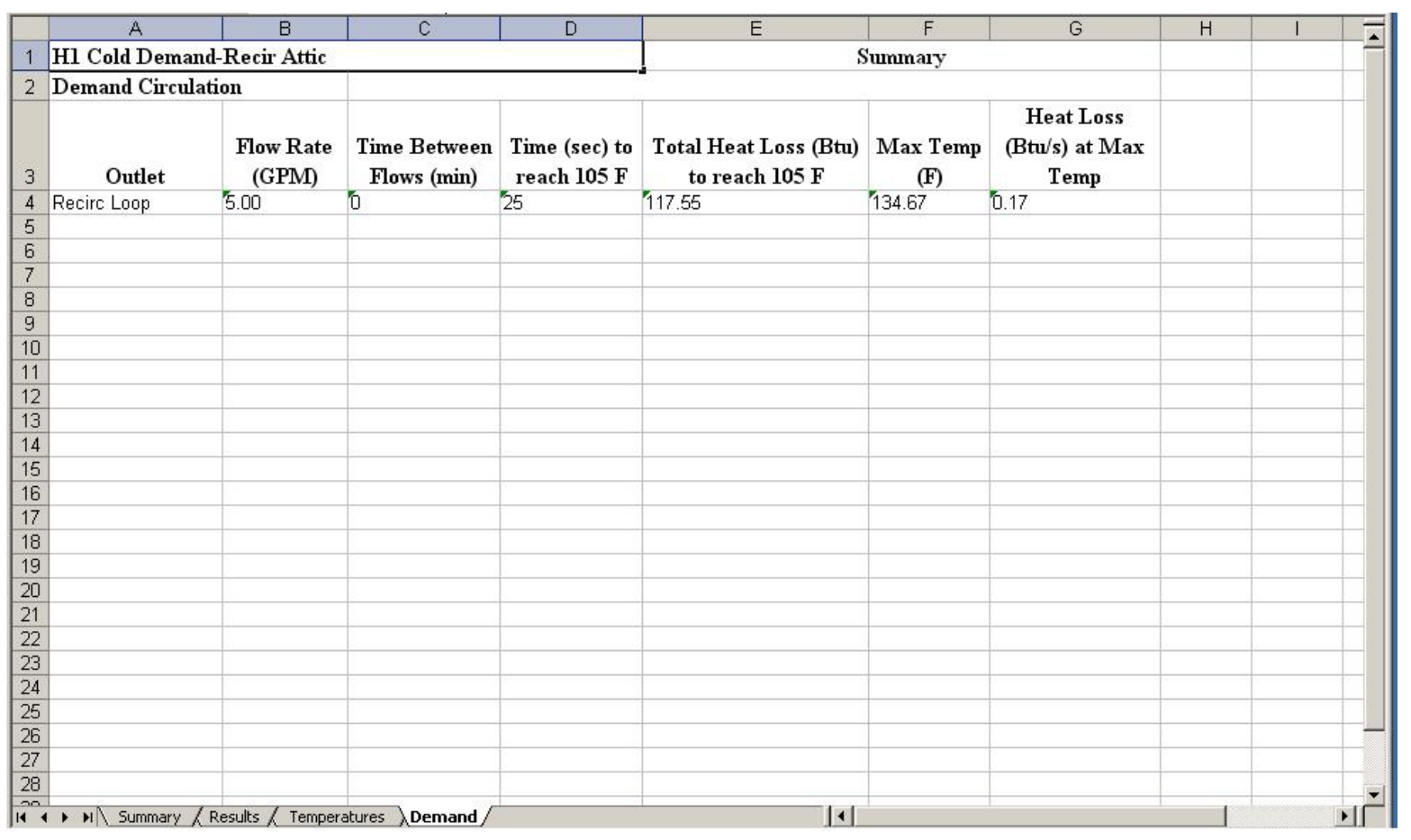

Fig. 3.13. Demand worksheet from results file for demand recirculation case.

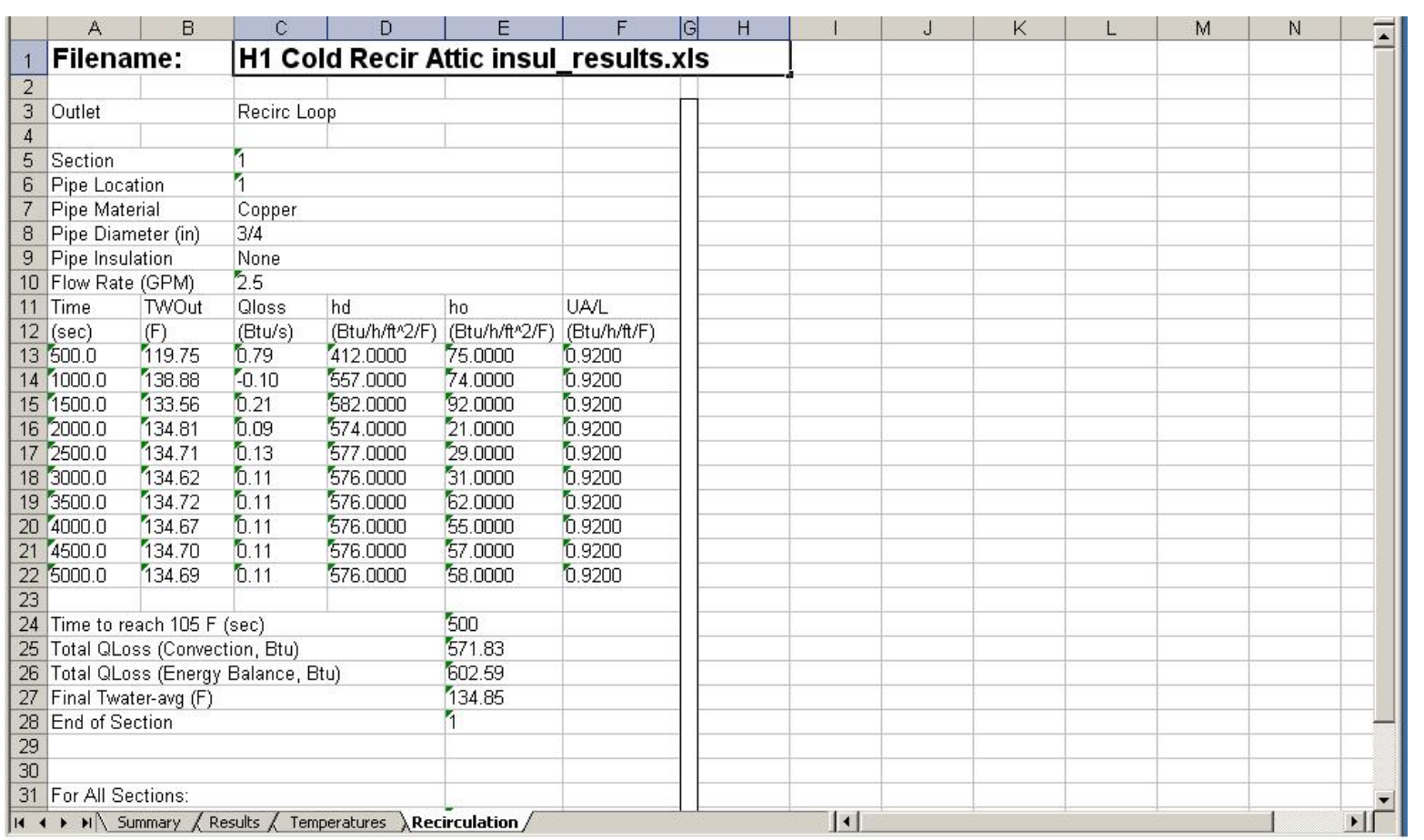

Fig. 3.14. Recirculation worksheet from results file for continuous recirculation case. 


\section{COMPARISONS TO EXPERIMENTAL DATA}

The comparisons of the calculational program to experimental data performed by Dr. Woodbury are documented in his report, which is included as Appendix $\mathrm{H}$. Briefly summarizing these results, the comparisons of the calculational model to 133 sets of experimental data for pipes in air were very good, particularly for the tests on copper pipe. The physical properties of the material for the tests with copper pipe are well known. The results for PEX-AL-PEX tests, though still good, were not as close as for copper pipe. In some cases the measured fluid temperature rose somewhat earlier than predicted, which may suggest that there is more axial mixing in the PEX-AL-PEX cases than for the copper pipe.

The comparison of calculational results to 21 sets of experimental data for copper pipe buried in wet sand produced good results for insulated pipe, but comparisons for bare copper pipe in wet sand led to the inclusion of a gap conductance model, to account for imperfect thermal between the pipe and its

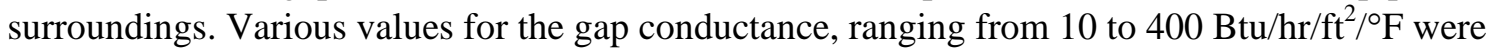
needed to obtain accuracies similar to those achieved for insulated, buried pipe, or pipe in air. For multiple tests conducted on the same day, lower gap conductance values were needed for later tests, consistent with degradation in the thermal contact between the pipe and sand as the day progressed. 



\section{CONSIDERATIONS IN MODELING RESIDENTIAL SYSTEMS}

In order to stay within the resources available to develop the computational tools needed to perform the original analyses, approximations were used to limit the complexity of the software development effort. These approximations could be reduced or removed if it was deemed worthwhile to devote additional resources towards software development. As noted above, the calculational program has been validated against experimental data. It is believed that the user-interface/computational program combination provides a reasonable tool to compare various hot water distribution system options. However, it is prudent to call attention to, and for the user to be aware of, some of the more noteworthy approximations and modeling considerations that derive there from.

For all systems other than the demand recirculation type, it has already been noted that when cooldown calculations are performed, to assess the effect of draws being near each other in time, the program assumes that the starting point for the cooldown calculation is the endpoint of the previous calculation. This means that for reasonable results, the modeler must call multiple uses at the same draw point in sequence, which may not correspond to the actual sequence. For example, suppose a cluster of 4 draws, two at each of two separate use points, separated by short intervals, is assumed to occur; represent this as A B A B. The modeler needs to enter these in the usage file as A A B B. The time delays for the arrival of hot water and energy losses should be calculated reasonably well, even though the modeled sequence varies from the actual one. The ability to allow the modeler to simply enter the actual sequence could be incorporated through further development of the user interface, by providing it with the capability to keep track of multiple previous draws instead of only referencing the immediately preceding draw.

As has been noted, the preceding discussion is moot for the demand recirculation system, as the program does not currently have the ability to provide a reasonable assessment of the effect of clustered draws for this type system. If additional development were done to provide the ability described in the previous paragraph along with the ability to control the duration of the calculation by temperature, rather than purely by time, clustered draw scenarios could be reasonably performed for demand recirculation systems. However, it is not clear if the market penetration of this type of system is sufficient to warrant the additional effort. 



\section{CONCLUSIONS}

Software has been developed to allow calculation of the time delay in the arrival of hot water at use points and of the energy lost from hot water distribution systems. The software consists of a calculational program produced by Dr. Keith Woodbury, and a user-interface produced by Dr. Roberto Lenarduzzi. The calculational program has been compared to experimental data produced by Dr. Carl Hiller and has demonstrated the ability to produce accurate results. The program produced consistently good results for all experimental configurations except for bare copper pipe buried in wet sand. In this latter case, the use of a gap conductance model allowed good comparisons to data, but the value of the gap conductance needed to match the data varied from experiment to experiment. It is believed that this represents variation in and uncertainty about the thermal contact between the bare pipe and the wet sand, as in the thermal properties of the sand itself. Descriptions of the calculational program and the user interface are provided herein, as well as instructions for their use. 



\section{REFERENCES}

1. R. Wendt, E. Baskin, and D. Durfee, "Evaluation of Residential Hot Water Distribution Systems by Numeric Simulation,” March 2004. http://www.ornl.gov/sci/res_buildings/FEMAattachments/hwds_eval_final_report.pdf

2. F. P. Incropera and D. P. DeWitt, Fundamentals of Heat and Mass Transfer, John Wiley and Sons, 2002.

3. B. K. Hodge and R. P. Taylor, Analysis and Design of Energy Systems, Prentice Hall, 1999.

4. K. H. Huebner, D. L. Dewhirst, D. E. Smith, and T. G. Byrom, The Finite Element Method for Engineers, 4th Edition, Wiley Interscience, 2001.

5. D. Pepper and J. Heinrich, The Finite Element Method: Basic Concepts and Applications, Hemisphere Publishing Company, 1992.

6. J. V. Beck, K. D. Cole, A. Haji-Sheikh, and B. Litkouhi, Heat Conduction using Green's Functions, Hemisphere, 1992. 



\section{Appendix A. DIRECTORY STRUCTURE}

The Executable software directory structure contains the following:

\begin{tabular}{|l|l|l|}
\hline HWD_v42.exe & File & Executable version of the user interface program. \\
\hline LVWUtil32.dll & File & Utility library file used by the interface program. \\
\hline HWD_v42.ini & File & $\begin{array}{l}\text { Initialization file for executable version of the user interface program; } \\
\text { created by the program when it executes. }\end{array}$ \\
\hline Calc & Directory & $\begin{array}{l}\text { Contains the calculational program and the text files used as input and } \\
\text { output. The names of the files are fixed and cannot be changed. The } \\
\text { files in this directory are ORNLpipe.exe, ORNLpipeGap.exe, infile.txt } \\
\text { and outfile.txt. The latter two are generated by the user interface. }\end{array}$ \\
\hline Excel Templates & Directory & $\begin{array}{l}\text { Contains Excel files used as templates by the user interface to read and } \\
\text { write information to and from Excel files. }\end{array}$ \\
\hline InputData & Directory & Contains the input data Excel files read by the user interface. \\
\hline Properties & Directory & $\begin{array}{l}\text { Contains pipe.xls, an Excel file that contains the dimensions and } \\
\text { properties of pipes, and insulation.xls, a file which contains the } \\
\text { material properties of insulation and material surrounding pipes (such } \\
\text { as clay, sand, etc.). }\end{array}$ \\
\hline UsageData & Directory & $\begin{array}{l}\text { Contains Excel files which define the order of events, times between } \\
\text { events and start temperatures for the events and segments. }\end{array}$ \\
\hline OutputData & Directory & $\begin{array}{l}\text { Contain sub-directories named after the simulation. Each sub-directory } \\
\text { contains the Excel file created by the user interface summarizing the } \\
\text { results of a calculation. If the user chooses to save intermediate files, } \\
\text { the input and output text files used by the calculational program are } \\
\text { also saved here. }\end{array}$ \\
\hline
\end{tabular}





\section{Appendix B. PIPE PROPERTIES FILE}

1. Filename must be "pipe.xls" (Excel Spreadsheet)

2. File must reside in the "properties" directory

3. Format

Column A pipe material

Column B Pipe nominal diameter (must be text) in inches

Column C pipe type

Column D pipe outside diameter (actual) in inches expressed as a decimal figure

Column E pipe inside diameter (actual) in inches expressed as a decimal figure

Column $\mathrm{F}$ thickness (h) of the pipe in inches expressed as a decimal figure

Column G specific heat (cp) of the pipe in Btu/lbm/ ${ }^{\circ} \mathrm{F}$

Column $\mathrm{H}$ Thermal conductivity (k) of the pipe in Btu/hr/ft/ ${ }^{\circ} \mathrm{F}$

Column I density (rho) of the pipe in $\mathrm{lbm} / \mathrm{ft}^{3}$

Column J emissivity (emiss) of the pipe

4. Entries in Columns A, B, and C are "text."

5. When generating an Input Data File, entries for the pipe material, diameter and type must be identical to the respective entries in Columns A, B and C.

Excerpt from Pipe.xls

\begin{tabular}{|l|l|l|r|r|r|r|r|r|r|}
\hline \multicolumn{1}{|c|}{$\mathbf{A}$} & \multicolumn{1}{c|}{ B } & \multicolumn{1}{c|}{ C } & \multicolumn{1}{c|}{ D } & \multicolumn{1}{c|}{ E } & \multicolumn{1}{c|}{ F } & \multicolumn{1}{c|}{ G } & \multicolumn{1}{c|}{ H } & \multicolumn{1}{c|}{ I } & \multicolumn{1}{c|}{ J } \\
\hline Material & Dia & Type & \multicolumn{1}{c|}{ OD } & ID & h & cp & k & rho & Emiss \\
\hline Copper & $1 / 4$ & K & 0.375 & 0.305 & 0.035 & 0.092 & 227 & 556 & 0.72 \\
\hline Copper & $3 / 8$ & K & 0.5 & 0.402 & 0.049 & 0.092 & 227 & 556 & 0.72 \\
\hline Copper & $1 / 2$ & K & 0.625 & 0.527 & 0.049 & 0.092 & 227 & 556 & 0.72 \\
\hline Copper & $5 / 8$ & K & 0.75 & 0.652 & 0.049 & 0.092 & 227 & 556 & 0.72 \\
\hline
\end{tabular}





\section{Appendix C. INSULATION PROPERTIES FILE}

1. Filename must be "insulation.xls" (Excel Spreadsheet)

2. File must reside in the "properties" directory

3. Format

Column A insulation material (this can be material used to insulate pipe, or a substance in which the pipe might be buried, such as clay or sand)

Column B location (currently defaults to "any")

Column C specific heat (cp) of the insulation in Btu $/ \mathrm{lbm} /{ }^{\circ} \mathrm{F}$

Column D thermal conductivity (k) of the insulation in Btu/hr/ft/ ${ }^{\circ} \mathrm{F}$

Column E density (rho) of the insulation in $\mathrm{lbm} / \mathrm{ft}^{3}$

Column F emissivity $(\varepsilon)$ of the insulation

4. Entries in Columns A, and B are "text."

5. When generating an Input Data File, entries for the insulation material must be identical to the respective entries in Columns A.

Insulation.xls

\begin{tabular}{|l|l|r|r|r|r|}
\hline \multicolumn{1}{|c|}{ A } & \multicolumn{1}{c|}{ B } & \multicolumn{1}{c|}{ C } & \multicolumn{1}{c|}{ D } & \multicolumn{1}{c|}{ E } & \multicolumn{1}{c|}{ F } \\
\hline Material & Type & \multicolumn{1}{l|}{ cp } & k & rho & \multicolumn{1}{c|}{ emiss } \\
\hline Calcium Silicate l & any & 0.0000413 & 0.028 & 15 & 0.87 \\
\hline Calcium Silicate ll & any & 0.0000413 & 0.028 & 22 & 0.87 \\
\hline Cellular Glass & any & 0.18 & 0.039 & 7.95 & 0.87 \\
\hline Mineral Fiber & any & 0.0000449 & 0.022 & 3 & 0.87 \\
\hline Mineral Fiber (loose fill) & any & 0.17 & 0.0208 & 1.3 & 0.87 \\
\hline
\end{tabular}





\section{Appendix D. INPUT DATA FILE}

1. Filename generated by user

2. Spreadsheet format with Excel extension (*.xls)

3. All entries in the spreadsheet are formatted to "text"

4. Format:

Column A Event ID—a number used by the program to refer to this line of input

Column B Outlet - a text description of the line of input

Column C Flowrate in gallons/minute

Column D Number of pipe segments in this event

Column E Pipe material-must match exactly with an entry in pipe.xls

Column F Pipe type-must match exactly with an entry in pipe.xls

Column G Pipe insulation-must match exactly with an entry in insulation.xls

Column H Use Gap Conductance-enter YES to invoke gap conductance model, otherwise enter NO

Column I Gap conductance for outside surface of pipe in Btu/hr/ $/ \mathrm{ft}^{2} /{ }^{\circ} \mathrm{F}$ (may be left blank if gap model is not used)

Column J Gap conductance for outside surface of insulation, if present, in $\mathrm{Btu} / \mathrm{hr} / \mathrm{ft}^{2} /{ }^{\circ} \mathrm{F}$ (may be left blank if gap model is not used)

For each segment there are 8 columns

Columns K, S, etc Pipe location-must be AIR, SOIL, or ATTIC

Columns L, T, etc. Surrounding material—whatever material surrounds the pipe, other than insulation applied to the pipe and already described in column G; must match exactly with an entry in insulation.xls

Columns M, U, etc. Thickness in inches of pipe's insulation that was described in column G

Columns N, V, etc. Surrounding material thickness in inches

Columns O, W, etc. Pipe nominal diameter (must be text) in inches—must match exactly with an entry in pipe.xls

Columns P, X, etc. Segment length, in feet

Columns $\mathrm{Q}, \mathrm{Y}$, etc. $\quad$ Temperature $\left({ }^{\circ} \mathrm{F}\right)$ of material surrounding the pipe; i.e., that described by columns L, T, etc.

Columns R, Z, etc. $\quad$ Initial temperature of water in pipe, ${ }^{\circ} \mathrm{F}$.

5. Pipe material and type are the same for all segments in the event.

6. Pipe insulation (if present) is the same for all segments in the event, although the thickness can vary from segment to segment; by entering 0 for the thickness, the absence of insulation can also be specified for a particular segment.

7. The column headings in this file occupy lines 1-3. The first line of data is on line 30 . The intervening lines, numbers 4-29, are generally hidden, and need not be used. This feature is included to allow the user to enter the allowable entries for those columns where a user entry must exactly match that of another file. (For example, the entries for pipe material, pipe type and pipe nominal diameter must exactly match an entry in the pipe.xls file. By copying the list of allowable entries from pipe.xls, and then using the "pick from list" option, the user can guard against typographical errors. The "pick from list option" is invoked by right clicking on the cell needing an entry, and then selecting this option.) As long as the program can find matches for the entries in this file in pipe.xls or insulation.xls, the program proceeds correctly, whether or not there are any entries in lines 4-29. This is simply an optional user convenience. 


\section{Example of Input Excel File}

\begin{tabular}{|c|c|c|c|c|c|c|c|c|c|c|}
\hline & $\mathbf{A}$ & B & $\mathbf{C}$ & D & $\mathbf{E}$ & $\mathbf{F}$ & $\mathbf{G}$ & $\mathbf{H}$ & $\mathbf{I}$ & $\mathbf{J}$ \\
\hline 1 & \multicolumn{10}{|c|}{ Distribution System Description and Parametric Input } \\
\hline 2 & Event ID & Outlet & Flowrate & Segments & $\begin{array}{c}\text { Pipe } \\
\text { Material }\end{array}$ & Pipe Type & Pipe Insul & $\begin{array}{c}\text { Use Gap } \\
\text { Conductance }\end{array}$ & $\begin{array}{c}\text { Gap } \\
\text { Conductance } \\
\text { Pipe }\end{array}$ & $\begin{array}{c}\text { Gap } \\
\text { Conductance } \\
\text { Insulation }\end{array}$ \\
\hline 3 & & & (GPM) & & $\begin{array}{c}\text { (pick from } \\
\text { list) }\end{array}$ & $\begin{array}{c}\text { (pick from } \\
\text { list) }\end{array}$ & (pick from list) & (pick from list) & (BTU/hr/ft' $/ F)$ & (BTU/hr/ft' $/ F)$ \\
\hline 30 & 1 & MBR shower & 2.25 & 2 & Copper & $\mathrm{M}$ & Cellular Polyethylene & $\mathrm{NO}$ & & \\
\hline 31 & 2 & MBR sink-1 & 1.25 & 2 & Copper & $\mathrm{M}$ & Cellular Polyethylene & NO & & \\
\hline 32 & 3 & $\begin{array}{l}\text { MBR } \\
\text { whirlpool }\end{array}$ & 0 & 2 & Copper & $\mathrm{M}$ & Cellular Polyethylene & NO & & \\
\hline 33 & 4 & MBR sink-2 & 1.25 & 2 & Copper & $\mathrm{M}$ & Cellular Polyethylene & NO & & \\
\hline 34 & 5 & MBR sink-1 & 1.25 & 2 & Copper & $M$ & Cellular Polyethylene & NO & & \\
\hline 35 & 6 & MBR sink-2 & 1.25 & 2 & Copper & $M$ & Cellular Polyethylene & NO & & \\
\hline 36 & 7 & BR2 shower & 2.25 & 2 & Copper & $M$ & Cellular Polyethylene & $\mathrm{NO}$ & & \\
\hline 37 & 8 & BR2 shower & 2.25 & 2 & Copper & $\mathrm{M}$ & Cellular Polyethylene & NO & & \\
\hline 38 & 9 & BR2 sink & 1.25 & 2 & Copper & $\mathrm{M}$ & Cellular Polyethylene & $\mathrm{NO}$ & & \\
\hline 39 & 10 & BR2 sink & 1.25 & 2 & Copper & $M$ & Cellular Polyethylene & NO & & \\
\hline
\end{tabular}


Example of Input Excel File (continued)

\begin{tabular}{|c|c|c|c|c|c|c|c|c|}
\hline & $\mathrm{K}$ & $\mathbf{L}$ & $\mathbf{M}$ & $\mathbf{N}$ & 0 & $\mathbf{P}$ & $\mathbf{Q}$ & $\mathbf{R}$ \\
\hline 1 & \multicolumn{8}{|c|}{ Section 1} \\
\hline 2 & $\begin{array}{c}\text { Pipe } \\
\text { Location }\end{array}$ & Surr Insul & pipe $_{\text {Insul-thick }}$ & Surr $_{\text {Insul-thick }}$ & Dia & Length & Tamb & Twater \\
\hline 3 & $\begin{array}{c}\text { (pick from } \\
\text { list) }\end{array}$ & (pick from list) & (in) & (in) & (in) & (ft) & (F) & (F) \\
\hline 30 & ATTIC & Mineral Fiber (loose fill) & 0 & 6 & $3 / 4$ & 64.5 & 76 & 76 \\
\hline 31 & ATTIC & Mineral Fiber (loose fill) & 0 & 6 & $3 / 4$ & 64.5 & 76 & 76 \\
\hline 32 & ATTIC & Mineral Fiber (loose fill) & 0 & 6 & $3 / 4$ & 64.5 & 76 & 76 \\
\hline 33 & ATTIC & Mineral Fiber (loose fill) & 0 & 6 & $3 / 4$ & 64.5 & 76 & 76 \\
\hline 34 & ATTIC & Mineral Fiber (loose fill) & 0 & 6 & $3 / 4$ & 64.5 & 76 & 76 \\
\hline 35 & ATTIC & Mineral Fiber (loose fill) & 0 & 6 & $3 / 4$ & 64.5 & 76 & 76 \\
\hline 36 & ATTIC & Mineral Fiber (loose fill) & 0 & 6 & $3 / 4$ & 37 & 76 & 76 \\
\hline 37 & ATTIC & Mineral Fiber (loose fill) & 0 & 6 & $3 / 4$ & 37 & 76 & 76 \\
\hline 38 & ATTIC & Mineral Fiber (loose fill) & 0 & 6 & $3 / 4$ & 37 & 76 & 76 \\
\hline 39 & ATTIC & Mineral Fiber (loose fill) & 0 & 6 & $3 / 4$ & 37 & 76 & 76 \\
\hline
\end{tabular}


Example of Input Excel File (continued)

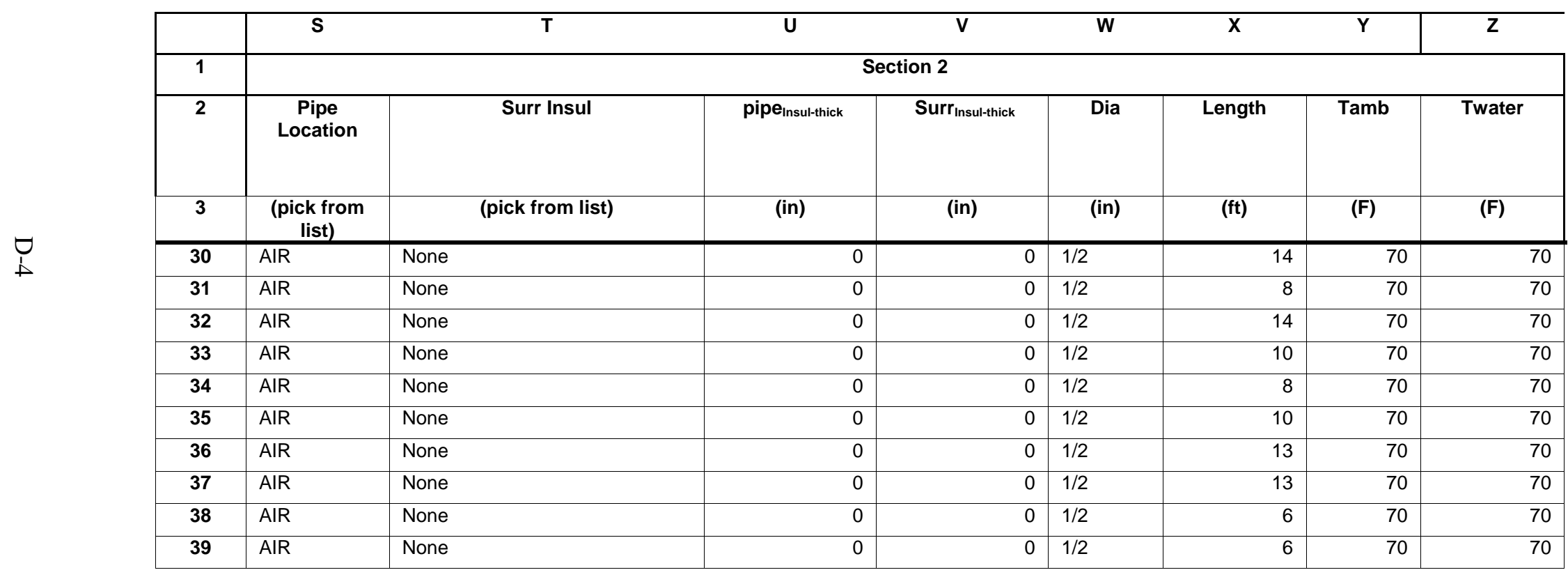




\section{Appendix E. USAGE DATA FILE}

1. An Excel file that can have any name chosen by the user

2. All entries in the spreadsheet are formatted to "text"

3. Format:

Column A Flow Usage - this column contains a number that will be used to pick the line from the input file with the corresponding "Event ID" number as the next event, in sequence, to occur. This column thus contains only numbers that are defined in the input file, but can contain any subset of those numbers, in any order, to define the sequence of events for this particular calculation.

Column B Outlet-text that labels the event; usually taken as the same as the text in column B of the input file, but this is not a necessity; the program is controlled by the event IDs specified in column A; this field is only for convenience

Column C Time before this event in minutes — defines a pause between events. If $>0$ the cooling in the segments is calculated, starting from the ending conditions of the previous event's calculation and cooling without flow is computed for the length of time specified by this entry.

Column E Number of segments (should be the same as the input data file)

Columns G, I, etc. Must be either Tamb or Tnew; this is a flag that controls which temperature is used for each segment at the start of the calculation: Tamb $=$ ambient temperature as defined in the input data file, Tnew = calculated temperature after the cooling period.

Excerpt from Usage File

\begin{tabular}{|c|c|c|c|c|c|c|c|}
\hline A & B & C & D & $\mathbf{E}$ & $\mathbf{F}$ & G & $\mathbf{H}$ \\
\hline \multicolumn{4}{|c|}{ Usage Data } & $\begin{array}{c}\text { Segment } \\
1 \\
\end{array}$ & $\begin{array}{c}\text { Segment } \\
2 \\
\end{array}$ & $\begin{array}{c}\text { Segment } \\
3 \\
\end{array}$ & $\begin{array}{c}\text { Segment } \\
4\end{array}$ \\
\hline $\begin{array}{l}\text { Flow } \\
\text { Usage }\end{array}$ & Outlet & $\begin{array}{l}\text { Time Before } \\
\text { Outlet Use } \\
\text { (min) }\end{array}$ & Segments & Temp Use & Temp Use & Temp Use & Temp Use \\
\hline 1 & MBR shower & 0 & 2 & Tamb & Tamb & & \\
\hline 2 & MBR sink-1 & 15 & 2 & Tnew & Tamb & & \\
\hline 4 & MBR sink-2 & 15 & 2 & Tnew & Tamb & & \\
\hline 7 & BR2 shower & 20 & 2 & Tnew & Tamb & & \\
\hline 8 & BR2 shower & 15 & 2 & Tnew & Tnew & & \\
\hline 9 & BR2 sink & 15 & 2 & Tnew & Tamb & & \\
\hline
\end{tabular}





\section{Appendix F. USE OF THE CORE CALCULATIONAL PROGRAM}

The LabVIEW user interface takes input provided by the user in Excel file format, converts it to text files in the format expected by the core calculational program, and calls the program repeatedly, generally once for each line specified in the usage Excel file. The usage file specifies the order of events, referencing each event by the number of the corresponding line in the input Excel file. The interface then provides the combined output from these calculations to the user in Excel format. One can, however, use the executable file for the core calculational program directly, by providing it with the appropriately formatted input text file. The following information describes the input text file used by and the output text file produced by the calculational program:

\section{Calculation Input File}

a. The calculational program expects an input text file in the same directory as the executable files (ORNLpipe.exe and ORNLpipeGap.exe). This input file is expected to have the name infile.txt. If you are using the user interface program, this file will be generated and written to the Calc subdirectory by the user interface.

b. The program writes its output to a text file in the same directory as the executable files. This output file is called outfile.txt.

c. When the user interface program is employed, both the infile.txt and outfile.txt files are written over for each event calculation. If the user selects the "keep" option under "intermediate files" on the first screen drawn by the interface program, the user interface generates identical input and output text files and stores them in the OutputData directory under the folder bearing the name of the Excel input file. In order to distinguish the text input and output files from one another, and to keep them from being overwritten, the naming convention used is "in_xx.txt" and "out_xx.txt" where $x x$ is a two digit representation of the event number (taken from the entry for each event in the first column of the input Excel file).

d. If the usage file specifies that the "Time Before Before Outlet Use" is greater than 0, the user interface will also cause a cooldown calculation to be performed before the event calculation itself is performed. This cooldown calculation uses zero flow, and takes as its initial conditions the ending conditions of the previous calculation. The input and output text files for cooldown calculations have the format "in_xxp.txt" and "out_xxp.txt" and are also saved in the OutputData directory under the folder bearing the name of the Excel input file.

e. The text file input is free format within each line, with the input values separated by any number of white spaces (blanks and tab characters). Trailing information on a line after the expected data has been read is ignored, so this can be used to include comments if desired, with the exception of keyword lines for each segment. No additional comments are allowed on the keyword lines.

f. The calculational program can do 3 types of calculations. Which type is done is controlled by the value in the 3rd line of input, which specifies the flow rate through the system. If this value is positive, a normal calculation assuming that flow rate is performed. If this value is zero, a calculation is performed that computes purely the conduction losses from the system. If this value is negative, a special computation is performed. The special computation assumes that the initial fluid temperature in the pipe is equal to the temperature specified in line 4 of the input, and the cooldown due to conduction is calculated. This latter capability is used by the user interface program to provide for cooling calculations between draws. 
g. Input Format:

i. Row 1 time step size and total time of calculation in seconds

ii. Row 2 label used to identify event (will be printed to output; since this is a string variable, all text on this line will be taken as part of the label and written to the output)

iii. Row 3 flow rate through the system in gallons per minute

iv. Row 4 inlet temperature in ${ }^{\circ} \mathrm{F}$

v. Row 5 number of segments in this event

vi. Row 6 inside diameter (inches) of pipe for each segment (number of entries on this line must match the number of segments specified in row 5)

vii. Row 7 outside diameter (inches) of pipe for each segment (number of entries on this line must match the number of segments specified in row 5)

viii. Row 8 insulation thickness (inches) for each segment (number of entries on this line must match the number of segments specified in row 5; 0 can be specified to indicate no insulation is present)

ix. Row 9 length (feet) of pipes for each segment (number of entries on this line must match the number of segments specified in row 5)

x. Row 10 pipe parameters: conductivity, $\mathrm{k}$, in Btu/lbm/ft/ ${ }^{\circ} \mathrm{F}$; density, $\rho$, in $\mathrm{lbm} / \mathrm{ft}^{3}$; specific heat, $\mathrm{c}_{\mathrm{p}}$, in Btu/lbm/ ${ }^{\circ} \mathrm{F}$, and emissivity of the outside of the pipe, $\varepsilon$.

xi. Row 11 insulation parameters: conductivity, $\mathrm{k}$, in Btu/lbm/ft/ ${ }^{\circ} \mathrm{F}$; density, $\rho$, in $\mathrm{lbm} / \mathrm{ft}^{3}$; specific heat, $\mathrm{c}_{\mathrm{p}}$, in Btu/lbm/ ${ }^{\circ} \mathrm{F}$, and emissivity of the outside of the pipe, $\varepsilon$. (this line must be present, even if no insulation exists; zeroes can be entered when no insulation is present)

[The next lines in the file consist of sets, with one set for each segment; thus, the number of sets equals that specified on line 5. Each set starts with a keyword line, that contains one of these three choices: AIR, ATTIC or SOIL. If AIR is specified, one additional line follows the keyword line. If ATTIC or SOIL are specified, two additional lines are required. The content and format of the lines following ATTIC or SOIL are identical. Below the requirements are given for an ATTIC/SOIL segment and an AIR segment. If more segments were specified, the input sets for the additional segments would follow, in order.]

xii. Row 12 keyword for first segment (assumed to be AIR for this presentation)

xiii. Row 13 ambient air temperature in ${ }^{\circ} \mathrm{F}$

xiv. Row 14 keyword for second segment (assumed to be ATTIC or SOIL)

$\mathrm{xv}$. Row 14 temperature $\left({ }^{\circ} \mathrm{F}\right)$ and thickness (inches) of the surrounding material (this might be loose fill cellulose for an attic, clay for a pipe buried in soil, etc.)

xvi. Row 15 parameters for the surrounding material: conductivity, $\mathrm{k}$, in $\mathrm{Btu} / \mathrm{lbm} / \mathrm{ft} /{ }^{\circ} \mathrm{F}$; density, $\rho$, in $\mathrm{lbm} / \mathrm{ft}^{3}$; specific heat, $\mathrm{C}_{\mathrm{p}}$, in $\mathrm{Btu} / \mathrm{lbm} /{ }^{\circ} \mathrm{F}$, and emissivity of the material, $\varepsilon$.

[Following the sets of input for each segment, 3 more lines of input are provided, as described below] 
xvii. Row 17 air velocity blowing across the outside of each segment in $\mathrm{ft} / \mathrm{sec}$ (these values will normally be zero, unless you have a pipe in AIR that is outdoors, or exposed in some fashion to wind; number of entries on this line must match the number of segments specified in row 5)

xviii. Row 18 initial fluid temperature for each segment in ${ }^{\circ} \mathrm{F}$ (number of entries on this line must match the number of segments specified in row 5)

[The last line of input is only required if ORNLpipeGap.exe is used. The user interface will use this executable if "YES" is specified in the "Use Gap Conductance" column of the Excel input file. If "NO" is specified, then ORNLpipe.exe is used, and the last line of input is not needed. The first number on this row is the gap conductance for the outside of the pipe (to either its insulation or to the surroundings if there is no insulation). If there is no insulation, only one number is used. The second number is the gap conductance for the outside of the insulation (to the surroundings).]

\section{xix. Row 19 Gap conductances in $\mathrm{Btu} / \mathrm{hr} / \mathrm{ft}^{2} /{ }^{\circ} \mathrm{F}$}

An example infile.txt for a case with two segments, the first in the attic and the second segment in air, for use with ORNLpipeGap.exe:

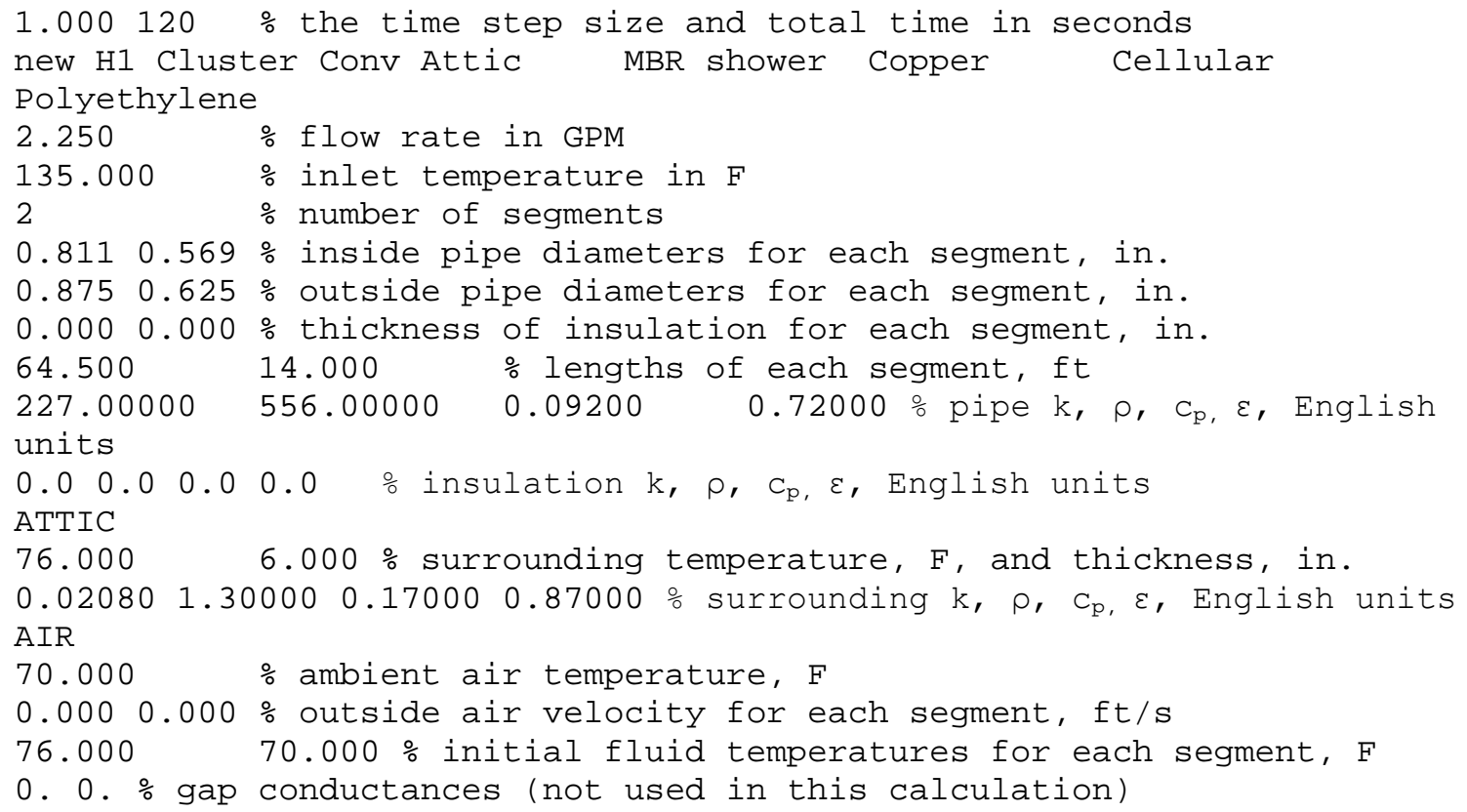

\section{Calculation Output File}

a. The calculational program writes its output to a text file in the same directory as the executable files. This output file is called outfile.txt. If you are using the user interface program, this file will be generated and written to the Calc subdirectory by the user interface.

b. When the user interface program is employed, both the infile.txt and outfile.txt files are written over for each event calculation. If the user selects the "keep" option under 
"intermediate files" on the first screen drawn by the interface program, the user interface generates identical input and output text files and stores them in the OutputData directory under the folder bearing the name of the Excel input file. In order to distinguish the text input and output files from one another, and to keep them from being overwritten, the naming convention used is "in_xx.txt" and "out_xx.txt" where $x x$ is a two digit representation of the event number (taken from the entry for each event in the first column of the input Excel file).

c. If the usage file specifies that the "Time Before Before Outlet Use" is greater than 0, the user interface will also cause a cooldown calculation to be performed before the event calculation itself is performed. This cooldown calculation uses zero flow, and takes as its initial conditions the ending conditions of the previous calculation. The input and output text files for cooldown calculations have the format "in_xxp.txt" and "out_xxp.txt" and are also saved in the OutputData directory under the folder bearing the name of the Excel input file.

d. The format of the output file consists of a block of output for each segment. Each block of output has the following format:
i. Row 1 Label (this echoes the text information provided on row 2 of infile.txt)
ii. Row 2 computed mass flow rate for the segment
iii. Row 3 computed fluid velocity for the segment
iv. Row 4 column labels
v. Row 5 units for the columns

[The columns of output consist of the following information: "segment" contains the number of the segment, counting the first one described in the input file as number 1 , the second as number 2, etc.; "time" provides the elapsed time from the start of the event; "Texit" provides the fluid temperature at the exit point for the segment; "Qloss" provides the heat loss rate from the fluid at that point in time, computed from an energy balance; "Q_hloss" provides the heat loss rate from the fluid at that point in time, computed from the fluid temperature and the convective heat transfer coefficient on the inside of the pipe; " $h D$ " provides the convective heat transfer coefficient on the inside of the pipe segment; "ho" provides the convective heat transfer coefficient on the outside of the pipe segment; "hrad" provides the radiation coefficient on the outside of the pipe]

\begin{tabular}{|c|c|c|}
\hline vi. & Rows 6-n & calculated data for each time for the segment \\
\hline vii. & Row $n+1$ & blank \\
\hline viii. & Row $n+2$ & time for this segment outlet to reach $105^{\circ} \mathrm{F}$ in seconds \\
\hline ix. & Row $n+3$ & blank \\
\hline $\mathrm{x}$. & Row $n+4$ & "For this segment:" \\
\hline xi. & Row $n+5$ & $\begin{array}{l}\text { total heat loss as calculated by summing the values for } \\
\text { Q_hloss for the segment (loss by convection) }\end{array}$ \\
\hline xii. & Row $n+6$ & $\begin{array}{l}\text { total heat loss as calculated by summing the values for } \\
\text { Q_loss for the segment (loss from energy balance) }\end{array}$ \\
\hline xiii. & Row $n+7$ & blank \\
\hline xiv. & Row $n+8$ & blank \\
\hline $\mathrm{XV}$. & Row $n+9$ & $\begin{array}{l}\text { average fluid temperature in this segment at the end of the } \\
\text { calculation period }\end{array}$ \\
\hline xvi. & Row $n+10$ & blank \\
\hline xvii. & Row $n+11$ & blank \\
\hline sviii & Row n+12 & "For this event:" \\
\hline
\end{tabular}


xix. $\quad$ Row $n+13$

total energy loss for this event determined by convection (for the first segment, this value and the value on row $n+5$ will be the same; for subsequent events it will be the total for all segments thus far; thus, for the last segment, this line provides the total for the entire draw)

$\mathrm{xx}$. Row $\mathrm{n}+14$ total energy loss for this event determined by energy balance (for the first segment, this value and the value on row $n+5$ will be the same; for subsequent events it will be the total for all segments thus far; thus, for the last segment, this line provides the total for the entire draw)

xxi. Row $\mathrm{n}+15$ blank

xxii. Row $\mathrm{n}+16$ blank

[This block of data is then repeated for each successive segment] 
A sample of outfile.txt for a two segment calculation is provided below:

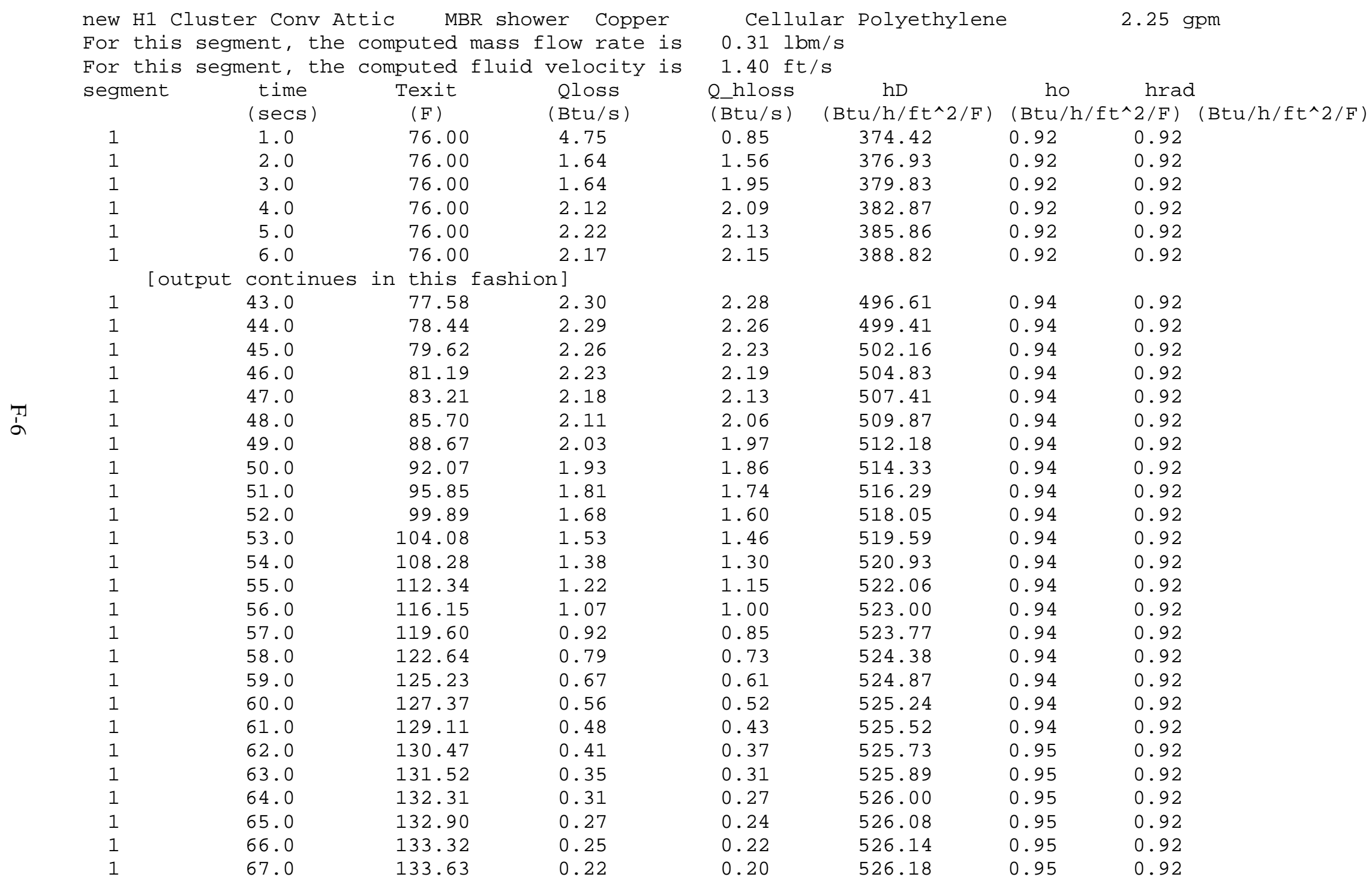




$\begin{array}{cccccccc}1 & 68.0 & 133.85 & 0.21 & 0.19 & 526.21 & 0.95 & 0.92 \\ 1 & 69.0 & 134.01 & 0.19 & 0.18 & 526.24 & 0.95 & 0.92 \\ 1 & 70.0 & 134.12 & 0.18 & 0.17 & 526.25 & 0.95 & 0.92 \\ & \text { [output continues } & \text { in this } & \text { fashion] } & & & & \\ 1 & 115.0 & 134.54 & 0.12 & 0.12 & 526.48 & 0.96 & 0.92 \\ 1 & 116.0 & 134.54 & 0.12 & 0.12 & 526.48 & 0.96 & 0.92 \\ 1 & 117.0 & 134.54 & 0.12 & 0.12 & 526.48 & 0.96 & 0.92 \\ 1 & 118.0 & 134.55 & 0.12 & 0.12 & 526.49 & 0.96 & 0.92 \\ 1 & 119.0 & 134.56 & 0.12 & 0.12 & 526.49 & 0.96 & 0.92 \\ 1 & 120.0 & 134.56 & 0.12 & 0.12 & 526.49 & 0.96 & 0.92\end{array}$

The time for this segment outlet to reach $105 \mathrm{~F}$ is $54.000 \mathrm{sec}$

For this segment:

Total heat loss by convection: $\quad 128.57$ Btu

Total heat loss by energy balance: 132.52 Btu

For this event:

Total heat loss by convection: $\quad 128.57$ Btu

Total heat loss by energy balance: 132.52 Btu

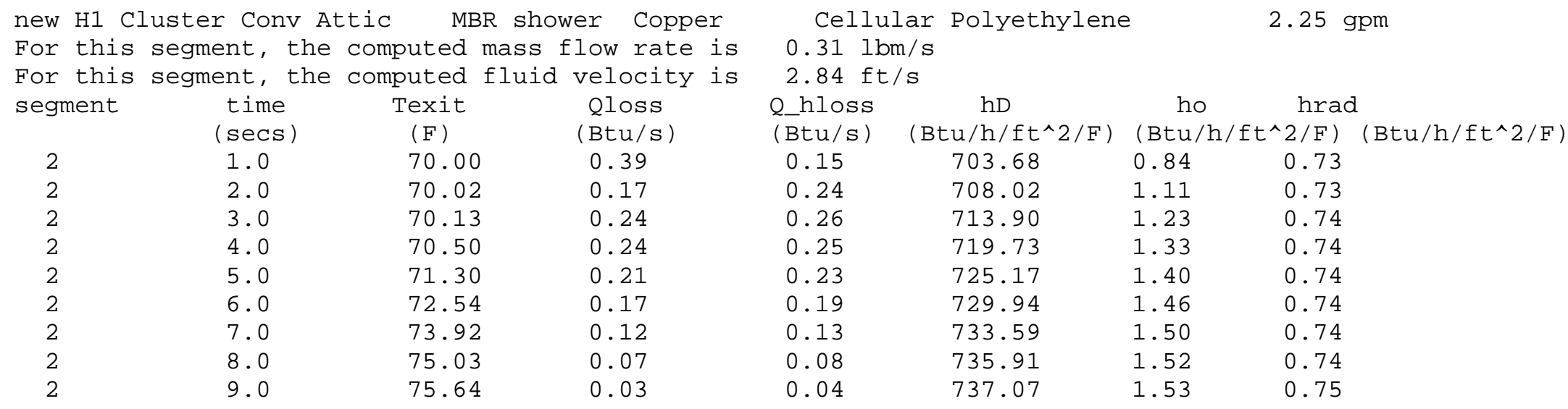




\begin{tabular}{|c|c|c|c|c|c|c|c|}
\hline 2 & 10.0 & 75.87 & $\odot .02$ & 0.02 & 737.53 & 1.54 & 0.75 \\
\hline 2 & 11.0 & 75.93 & $\odot .01$ & 0.01 & 737.68 & 1.54 & 0.75 \\
\hline 2 & 12.0 & 75.96 & 0.01 & 0.01 & 737.73 & 1.54 & 0.75 \\
\hline 2 & 13.0 & 75.97 & 0.01 & 0.01 & 737.77 & 1.54 & $\odot .75$ \\
\hline 2 & 14.0 & 75.98 & 0.01 & 0.01 & 737.78 & 1.54 & 0.75 \\
\hline & [output cc & in thi & fashion] & & & & \\
\hline 2 & 47.0 & 76.86 & 0.31 & 0.25 & 749.91 & 1.59 & 0.75 \\
\hline 2 & 48.0 & 77.36 & $0.4 \odot$ & 0.34 & 755.48 & 1.61 & 0.75 \\
\hline 2 & 49.0 & 78.08 & 0.51 & 0.43 & 762.80 & 1.63 & 0.75 \\
\hline 2 & 50.0 & 79.07 & 0.62 & 0.54 & 772.08 & 1.66 & 0.76 \\
\hline 2 & 51.0 & 80.39 & 0.75 & 0.65 & 783.44 & 1.70 & 0.76 \\
\hline 2 & 52.0 & 82.09 & 0.86 & 0.76 & 796.85 & 1.74 & 0.77 \\
\hline 2 & 53.0 & 84.21 & 0.97 & 0.87 & 812.16 & 1.78 & 0.77 \\
\hline 2 & 54.0 & 86.76 & 1.06 & 0.95 & 829.08 & 1.82 & 0.78 \\
\hline 2 & 55.0 & 89.73 & 1.13 & 1.02 & 847.16 & 1.86 & 0.79 \\
\hline 2 & 56.0 & 93.08 & 1.17 & 1.06 & 865.89 & 1.91 & 0.79 \\
\hline 2 & 57.0 & 96.75 & 1.18 & 1.07 & 884.70 & 1.95 & 0.80 \\
\hline 2 & 58.0 & 100.65 & 1.16 & 1.05 & 903.06 & 1.99 & 0.81 \\
\hline 2 & 59.0 & 104.65 & 1.10 & 1.00 & 920.45 & 2.02 & 0.82 \\
\hline 2 & 60.0 & 108.64 & 1.03 & 0.93 & 936.50 & 2.06 & 0.83 \\
\hline 2 & 61.0 & 112.51 & 0.93 & 0.84 & 950.91 & 2.08 & 0.84 \\
\hline 2 & 62.0 & 116.13 & 0.83 & 0.75 & 963.52 & 2.11 & 0.85 \\
\hline 2 & 63.0 & 119.43 & 0.72 & 0.65 & 974.29 & 2.13 & 0.85 \\
\hline 2 & 64.0 & 122.35 & 0.61 & 0.55 & 983.29 & 2.15 & 0.86 \\
\hline 2 & 65.0 & 124.86 & 0.52 & 0.47 & 990.63 & 2.16 & 0.86 \\
\hline 2 & 66.0 & 126.96 & 0.43 & 0.39 & 996.49 & 2.18 & 0.87 \\
\hline 2 & 67.0 & 128.67 & 0.35 & 0.32 & 1001.09 & 2.18 & 0.87 \\
\hline 2 & 68.0 & 130.03 & 0.29 & 0.27 & 1004.63 & 2.19 & 0.87 \\
\hline 2 & 69.0 & 131.09 & 0.24 & 0.22 & 1007.30 & 2.20 & 0.87 \\
\hline 2 & 70.0 & 131.90 & 0.20 & 0.19 & 1009.29 & 2.20 & 0.87 \\
\hline 2 & 71.0 & 132.51 & 0.17 & 0.16 & 1010.75 & 2.20 & 0.88 \\
\hline 2 & 72.0 & 132.96 & 0.15 & 0.14 & 1011.82 & 2.21 & 0.88 \\
\hline 2 & 73.0 & 133.28 & 0.13 & 0.13 & 1012.58 & 2.21 & 0.88 \\
\hline 2 & 74.0 & 133.52 & 0.12 & 0.12 & 1013.13 & 2.21 & 0.88 \\
\hline 2 & 75.0 & 133.69 & 0.11 & 0.11 & 1013.53 & 2.21 & 0.88 \\
\hline 2 & 76.0 & 133.81 & 0.11 & 0.10 & 1013.81 & 2.21 & 0.88 \\
\hline 2 & 77.0 & 133.90 & 0.10 & 0.10 & 1014.02 & 2.21 & 0.88 \\
\hline 2 & 78.0 & 133.96 & 0.10 & 0.10 & 1014.17 & 2.21 & 0.88 \\
\hline 2 & 79.0 & 134.00 & 0.10 & 0.10 & 1014.29 & 2.21 & 0.88 \\
\hline
\end{tabular}




$\begin{array}{rrrrrrrr}2 & 80.0 & 134.04 & 0.10 & 0.09 & 1014.37 & 2.21 & 0.88 \\ 2 & 81.0 & 134.06 & 0.10 & 0.09 & 1014.44 & 2.21 & 0.88 \\ 2 & 82.0 & 134.08 & 0.10 & 0.09 & 1014.50 & 2.21 & 0.88 \\ 2 & 83.0 & 134.10 & 0.09 & 0.09 & 1014.55 & 2.21 & 0.88 \\ 2 & 84.0 & 134.11 & 0.09 & 0.09 & 1014.59 & 2.21 & 0.88 \\ 2 & 85.0 & 134.12 & 0.09 & 0.09 & 1014.63 & 2.21 & 0.88 \\ & \text { [output continues in this } & \text { fashion] } & & & & \\ 2 & 115.0 & 134.24 & 0.09 & 0.09 & 1015.03 & 2.21 & 0.88 \\ 2 & 116.0 & 134.24 & 0.09 & 0.09 & 1015.03 & 2.21 & 0.88 \\ 2 & 117.0 & 134.24 & 0.09 & 0.09 & 1015.03 & 2.21 & 0.88 \\ 2 & 118.0 & 134.24 & 0.09 & 0.09 & 1015.03 & 2.21 & 0.88 \\ 2 & 119.0 & 134.24 & 0.09 & 0.09 & 1015.04 & 2.21 & 0.88 \\ 2 & 120.0 & 134.24 & 0.09 & 0.09 & 1015.06 & 2.21 & 0.88\end{array}$

The time for this segment outlet to reach $105 \mathrm{~F}$ is $60.000 \mathrm{sec}$

For this segment:

Total heat loss by convection: 22.59 Btu

Total heat loss by energy balance: $\quad 24.59$ Btu

For this event:

Total heat loss by convection:

$151.16 \mathrm{Btu}$

Total heat loss by energy balance:

157.11 Btu 



\section{Appendix G. ORNLpipe: PROGRAM DOCUMENTATION}

The following description of the programming approach used by Dr. Keith Woodbury was provided prior to the inclusion of the gap conductance model. A description of the comparison to experimental data and the subsequent development of the gap conductance model is provided in Appendix $\mathrm{H}$.

\section{ORNLpipe: PROGRAM DOCUMENTATION}

\section{main()}

The driver section of the program and many utility functions are defined in the source code module RunAllPipeTrans.cpp. The overall program flow in the main() of that module is illustrated in the flowchart in Fig. G.1. In this section the major function calls from main are described in the order in which they are called. For each routine, a operational description is given. In subsequent sections these routines may be described in more detail.

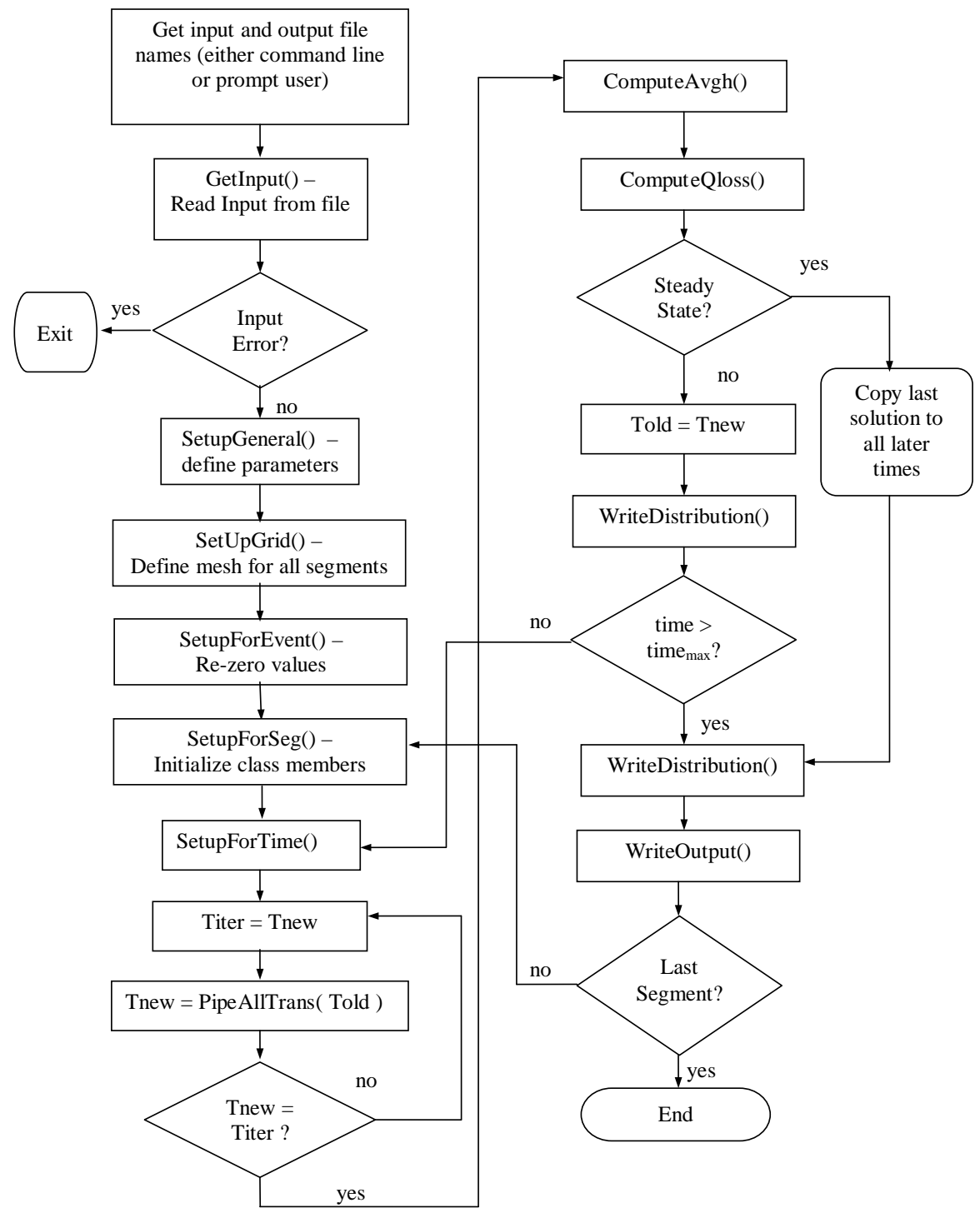

Fig. G.1. Overall program flow in main() of RunPipeAllTrans.cppMain Program Flow. 


\section{PipeAll::GetFileNames( int argc, char **argv )}

This routine accepts as input the argc and argv[] values passed to the main() from the command line. The name of the input and output files may be passed to the executable program on the command line. GetFileNames() checks to see if one or both of these parameters were supplied to the calling program and prompts the user to enter either one or both of these file names.

\section{PipeAll::GetInput( void )}

This function reads all the input required for the piping system analysis. The format for the input is free, with input values separated by any number of white spaces (blanks and tab characters). Also, trailing information on a line after expected data are ignored, so this region can be used to record helpful comments about each input line. A sample input file is shown in Table G.1 below and will be used as an example to describe the input process.

Table G.1. Sample input file

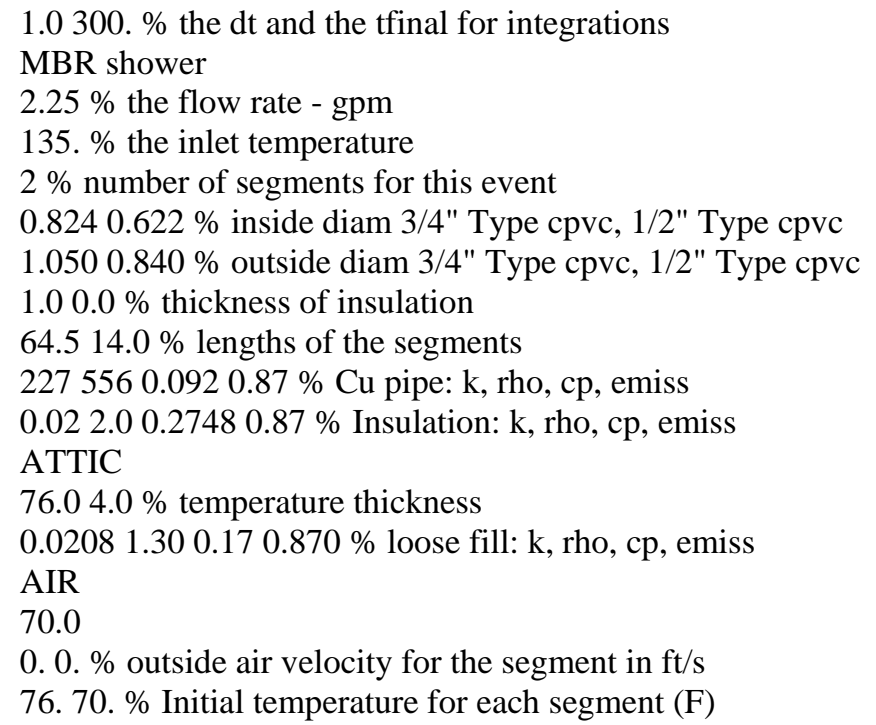

The first line contains the time step (in seconds) for integrations and the total simulation time (in seconds). In the example, computations are performed every 1 . second for a total of 300 . seconds.

The second line contains a string that is used to identify this event. Here a label of "MBR Shower" is used to identify this case. This string is printed to the output to associate the results with the data.

The third line specifies the flow rate through the system in GPM. If the flow rate is positive, then a "normal" pipe system computation is performed: the water temperature in the pipe is initialized to the temperature of the environment, then the temperatures in and around the pipe are computed as a function of time. If the flow rate is specified as zero, then a simulation of the system with no fluid flow will be performed (pure conduction). For this case, the initial fluid temperature is taken as that of the environment. If the flow rate is specified as any value less than zero, a special computation is performed whereby the initial fluid temperature is set equal to the supply temperature, and the heat loss during the cool-down is computed. This latter computation is also a pure conduction simulation. For the example file in Table G.1, a normal computation with 2.25 GPM will be performed.

The fourth line specifies the inlet (supply) water temperature in degrees Fahrenheit. 
The fifth input line specifies the number of segments in this event. Multiple segments are used to specify different diameter pipes and/or different environments for different segments of the piping system. The example system has 2 segments.

The sixth line specifies the inside diameter (in inches) of each segment. Note there must be exactly as many diameters on this line as number of segments specified on line five. The seventh line contains the outside diameters (in inches) for each segment.

The eighth line specifies the thickness of insulation (in inches) for each segment. Note that these must be specified, even if the thickness is zero, and that there must be exactly as many thicknesses on this line as number of segments specified on line five.

The ninth line contains the length (in feet) of each of the segments. Note there must be exactly as many lengths on this line as number of segments specified on line five.

The tenth line contains the thermophysical properties of the pipe material; and these will be the same for each segment in the event. This line must contain the thermal conductivity, $k$, in Btu/lbm/ft/ ${ }^{\circ} \mathrm{F}$, the density, $\rho$, in $\mathrm{lbm} / \mathrm{ft}^{3}$, the specific heat, $c_{\mathrm{p}}$, in Btu $/ \mathrm{lbm} /{ }^{\circ} \mathrm{F}$, and the emissivity of the outer surface of the pipe, $\varepsilon$, which is dimensionless.

The eleventh line contains the thermophysical properties of the piping insulation. The number of and order of and units of the properties are the same as on line ten. Note that this line must be present even if there is no insulation on the pipe (values of zero can be used if no insulation is present).

The remainder of the file is composed of sections, with one section present for each segment, which specify the environment for each section. Each section begins with a keyword, which must be one of ATTIC, SOIL, or AIR. Note that no trailing comments are allowed on the keyword line and that the case of the keyword is important. The ATTIC and SOIL keywords have exactly the same effect and either can be used. If the keyword ATTIC or SOIL is specified for a segment, there must be two additional lines in that segment's section. The first additional line specifies the temperature of the material surrounding the pipe (the environment temperature) and the thickness of the material surrounding the pipe. The second line contains the thermophysical properties of the material surrounding the pipe. The number of and order of and units of the properties are the same as on line ten and eleven. If the keyword AIR is used, then only one additional line must appear in that segment's section, and that additional line contains the temperature (in degrees F) of the air surrounding the pipe.

The next-to-last line in the file must contain the wind velocity (in $\mathrm{ft} / \mathrm{s}$ ) blowing across the outside of the pipe for each segment. These entries are typically zero, unless the piping system is exposed to an outdoor environment. Note there must be exactly as many velocities on this line as number of segments specified on line five.

The last line of the file contains the initial temperature of the fluid in each segment. Note there must be exactly as many temperatures on this line as number of segments specified on line five. If there is only one event in the file, this line is optional and the environment temperature is used for this initial condition. 


\section{PipeAll::SetupGeneral(void)}

In this routine a few overall parameters are computed or set. Specifically, the number of time steps is computed based on the values of tfinal and $d t$ read in from the input file. Also, the theta parameter for the time integration is set to a value of $2 / 3$, and the maximum number of iterations itmax is set to 500 , and the tolerance for convergence tol is set to 0.01 .

\section{PipeAll::SetupGrids(int event)}

This routine runs once for every event (not segment) read from the input file and generates the grid for each segment in the event. The number of axial locations $n x$ and the axial coordinates of the nodes $x$ are for each segment are stored in the class variables nxvec and xmat. Similarly, the number of radial node locations $n r$ and their radial coordinates $r$ for each segment are stored in the class variables nrvec and rmat. Note that the number of radial locations $n r$ does NOT include the fluid node, only the nodes on the pipe and in the surroundings.

A nonuniform grid is used in both the axial and radial direction. In the axial direction, a geometric progression is used to increase the grid from some minimum size $d x 0$ up to a desired fixed size $d x$. That is, $\Delta \mathrm{x}_{\mathrm{i}+1}=d x \operatorname{ratio}\left(\Delta \mathrm{x}_{\mathrm{i}}\right)$. By experimentation, the values of $d x 0=0.0025$ inch, dxratio $=1.2$, and $d x=4$ inches were determined to be satisfactory. The main criterion for these parameters was the repeatability of the heat loss computation for a no flow condition (the heat flow by conduction depends on the gradient of the temperature, and the small initial grid is needed to obtain a good approximation of the derivative in the first element).

In the radial direction, a uniform increment in the pipe wall is used. A desired element thickness in the pipe wall is set in the program, somewhat arbitrarily, to a value of $d r 1 d=0.01$ inches. This desired element thickness is modified accordingly to obtain an integral number of nodes through the pipe thickness. This results in a few (approximately 4 to 10) elements through most pipe thicknesses. If there is insulation present, a nonuniform grid is used in this portion, with the initial thickness equal to the thickness from the pipe wall. The geometric progression is used to stretch the grid, as in the axial case, and a nominal stretching ratio of ratio2 $=1.50$ is used. That is, $\Delta \mathrm{r}_{\mathrm{i}+1}=\operatorname{ratio} 2\left(\Delta \mathrm{r}_{\mathrm{i}}\right)$. The actual ratio is modified slightly to result in an integral number of increments through the insulation thickness. If there is surrounding material around the bare or insulated pipe (corresponding to the ATTIC or SOIL keyword for the environment type in the input file) then a nonuniform grid is used in that portion also. Again, the initial radial interval is taken to be the same as the last interval in the prior portion (either from the pipe, if bare, or from the insulation, if covered), and a nominal stretching ratio of ratio3 $=1.50$ (that is, $\Delta \mathrm{r}_{\mathrm{i}+1}=\operatorname{ratio} 3\left(\Delta \mathrm{r}_{\mathrm{i}}\right)$. This nominal value is adjusted slightly to obtain an integral number of nodes in the radial direction.

This routine also must determine which of the possible materials is actually on the outside of the domain: pipe, insulation, or SOIL/ATTIC. Then the corresponding value of emissivity is set in the class variable emmissmat to be recalled later for each segment.

\section{PipeAll::SetupForEvent(int event)}

This routine prepares for computations for a new event. Specifically, this routine

- Sets the class variable $Q$ flow to the value for this event from the vector flowrate (which was populated from the input file).

- Zeros QLoss_All and QLoss_hAll which are used to compute the total heat loss from the pipe for this event. 
- Sets the class variable Tin (the water supply temperature) for this event from the vector Tinp (which was populated from the input file).

- Computes the mass flow rate through the system for this event $m d o t=\rho Q$ flow (the density is taken at the temperature Tin where the fluid enters the system).

- Creates space for Texit which will hold the value of the exit temperature at each time step (Texit will be needed to supply the inlet temperature for the next segment in this event).

- Create space for time_ss which will be used to hold the time required to reach steady state for each segment in this event. time_ss[1] is set to zero.

- If an output file is used, and this is the first event, this output file is opened. This file remains open during this entire execution of the program.

\section{PipeAll::SetupForSeg(int event, int seg)}

This routine is executed once for every segment in the event and loads the appropriate values from the input into the class variables. Specifically, this routine.

- Creates space for the thermal conductivity kpipe and density specific heat product Cpipe for the variation of these properties in the radial direction.

- Initializes the Tinf variable from vector Tenviron (which is populated from the input).

- Creates space Tinlet to store the inlet water temperature for each time step. If $s e g>1$, then these values are initialized to those stored in Texit from the previous segment.

- Initializes class variables $n x, n r, r 1, r 2, r 3, r 4$ which are the number of axial nodes, number of radial nodes, radius of pipe inner diameter, radius of pipe outer diameter, radius of outside of insulation, and radius of outside of domain.

- Initializes the diam class variable, which is the inside pipe diameter

- Computes the class variable Vel_fluid $=Q$ flow $/\left(\pi \mathrm{diam}^{2} / 4\right)$.

- Create space for $r$ and $x$, the radial and axial coordinates, and initializes these from xmat and rmat [created in routine SetupGrids()].

- creates space for the length of the elements in the axial direction, lelem, and initializes these based on the $x$ variable.

- Creates space for ho, $h D$, and hrad, the heat transfer coefficients on the outside of the pipe, the inside of the pipe, and the radiation coefficient on the outside of the pipe. There is one each of these for each element along the axis.

- $\quad$ Populates the kpipe and Cpipe variables based on the material composition in the radial direction.

- Initializes the emiss class variable for the external surface emissivity based on the input data and the analysis in SetupGrids().

- Creates space for and initializes to zero several arrays:

o Tplot — will contain the fluid distribution along the pipe for all times

o Qplot - will contain the instantaneous heat flux from the pipe at each time based on an energy balance 
o q_hplot-will contain the instantaneous heat flux from the pipe at each time based on the heat transfer coefficient

o qAplot-will contain the "apparent" heat loss, $\dot{m} c_{p}\left(T_{\text {in }}-T_{\text {out }}\right)$

o hoplot-will contain the average ho along the pipe at each time

o hradplot - will contain the average hrad along the pipe at each time

o $h D p l o t$ - will contain the average $h D$ on the inside of the pipe at each time

- creates space for several arrays:

o $\quad T f$ - the fluid temperature along the pipe at each time

o Tfdot - the rate of change of temperature along the pipe at each instant

o Toutside - the temperature on the outside of the pipe at each time

o kf, rhof, cpf, muf, Prf-the conductivity, density, specific heat, dynamic viscosity, and Prandl number of the fluid along the pipe

- creates, initializes, and returns the Told variable, which is the initial temperature in the pipe. If $m d o t \geq 0$, then Told is initialized to Tinf. If $m d o t<0$, then Told is initialized to the supply temperature Tin.

\section{Integration Loop}

The central function of the program is accomplished by the time integration loop in the main(). This loop sequentially computes the temperature distribution in the fluid and in the surrounding pipe/insulation/soil and then computes the heat loss for each time step.

Since the solution for the fluid temperatures depends on the fluid temperatures (because the properties of the fluid are temperature dependent), an iteration loop is generally required to determine the appropriate temperatures and properties. Although the program structure is in place for such iteration, the need for iterations is virtually eliminated through linearization of the properties. This linearization is accomplished by computing the properties at the temperature from the previous time step.

The primary function that computes the temperatures for the next time step is PipeAll::PipeAllTrans(). This large and important function will be documented in a separate section. Once the new temperatures are computed, the average heat transfer coefficient along the pipe is computed via PipeAll::ComputeAvgh() and the heat loss is computed via PipeAll::ComputeQloss() for the current time.

\section{PipeAll::ComputeAvgh(int itime)}

This simple function computes the average heat transfer coefficient on the outside of the pipe for the current time step, ho, and stores this value in the appropriate location in hoplot. Similarly, the average radiation coefficient hrad and average heat transfer coefficient on the inside of the pipe $h D$ are computed and stored in their respective locations hradplot and hDplot.

A simple arithmetic average is used to compute the average value; it is not a surface area average. The values along the pipe needed to compute the average were computed previously in the routine PipeAll::PipeAllTrans(). 


\section{PipeAll::ComputeAvgTf( int itime )}

This function computes the average temperature of the fluid in the current segment for the specified itime index. A volume-averaged temperature is computed, rather than the strict numerical average, since a variable grid is used in the $x$ direction. The main purpose of this function is to provide the input for a subsequent simulation following a "cool-down” simulation.

\section{PipeAll::ComputeQloss(int itime, CMatrix \& Told, CMatrix \& Tnew)}

This routine evaluates the instantaneous rate of heat loss from the pipe by two separate calculations. One computation is based on an energy balance, and the second is computed from the heat loss by convection to the pipe wall.

Energy Balance Computation: A thermodynamic energy balance on the fluid in the pipe at any instant yields:

$$
\begin{aligned}
\text { advection }_{\text {in }}+\text { conduction }_{\text {in }} & =\text { advection }_{\text {out }}+\text { conduction }_{\text {out }}+ \\
& \text { Rate of energy increase in pipe }+ \text { Heat Loss from pipe }
\end{aligned}
$$

or

$$
\begin{aligned}
\dot{m} c_{p} T_{\text {in }}-\left.k A \frac{d T}{d x}\right|_{\text {in }} & =\dot{m} c_{p} T_{\text {out }}-\left.k A \frac{d T}{d x}\right|_{\text {out }}+\int_{\text {inlet }}^{\text {outlet }} \rho c_{p} \frac{d T}{d t} A d x+\dot{Q}_{\text {loss }} \\
\dot{m} c_{p} T_{1}-k A \frac{\left(T_{2}-T_{1}\right)}{\ell e_{1}} & =\dot{m} c_{p} T_{\mathrm{nx}}-k A \frac{\left(T_{\mathrm{nx}}-T_{\mathrm{nx}-1}\right)}{\ell e_{(\mathrm{nx}-1)}}+\sum_{i=1}^{\mathrm{nx}-1} \rho c_{p} A(\ell e)_{i} \dot{T} e_{i}+\dot{Q}_{\text {loss }}
\end{aligned}
$$

Here $\ell e$ is the length of an element, and $\dot{T} e$ is the time rate of increase of the temperature of the fluid in the element. In the program, only the advective terms are used to compute the heat loss if the flow is non-zero (the conduction terms are neglected), and if the flow rate is zero, then only the conduction terms appear.

The value of $\dot{Q}_{\text {loss }}$ computed via the energy balance is stored in the class variable qplot and will be used at the end of the integration loop to compute the total heat loss from the fluid over the simulation time.

Convective heat loss computation. The heat loss from the pipe is only via convection from the fluid to the pipe wall. So at each instant of time, the heat loss from the fluid can be computed by integrating the convective heat loss along the length of the pipe:

$$
\begin{aligned}
\dot{Q}_{\text {loss }} & =\int_{\text {inlet }}^{\text {outlet }} \pi D h\left(T_{f}-T_{\text {wall }}\right) d x \\
& =\sum_{i=1}^{\text {nx }-1} \pi D h_{i}\left(T_{f}-T_{\text {wall }}\right) l e_{i}
\end{aligned}
$$

Here $T_{f}$ is the average temperature of the fluid in an element and $T_{\text {wall }}$ is the average pipe wall temperature adjacent to the element. These values have been previously computed for the current time step in the routine PipeAll::PipeAllTrans(). 
The value of $\dot{Q}_{\text {loss }}$ computed via the convective loss is stored in the class variable $q$ hplot and will be used at the end of the integration loop to compute the total heat loss from the fluid over the simulation time.

\section{PipeAll::CheckConverge(seg, itime, CMatrix \& Told, CMatrix \& Tnew)}

This routine checks for steady state conditions. Steady state is declared when the sum of the squared differences between Told and Tnew falls below the value of tol (read during input and stored in the class variable). If steady state conditions are declared, then this routine copies the last computed values of the appropriate variables into Texit, qplot, qAplot, q_hplot, hDplot, hoplot, and hradplot. If steady state conditions are declared, a TRUE signal is returned, otherwise FALSE is returned.

\section{PipeAll::WriteDistribution(CMatrix \& Tnew)}

This routine writes out the spatial distribution of temperature and heat transfer coefficients. This routine has been used for debugging, and presently is used to write the final distribution to the screen for the user's information. The nodal temperature of the fluid and the pipe wall and the outer pipe surface are written for each $x$ location, along with the elemental values of heat transfer coefficients ( $h D$, ho, and hrad).

\section{PipeAll::WriteOutput(CMatrix \& Tnew)}

This routine writes the output to the file for a permanent record. The output file was previously opened in the SetupForEvent() routine. Information previously stored that is written out includes Texit, qplot, q_hplot, hDplot, hoplot, and hradplot.

The total heat loss is also computed in this routine by integrating the instantaneous heat loss over time. A trapezoidal integration scheme is used on the discrete data:

$$
\begin{aligned}
\text { Qloss } & =\int_{0}^{\text {tfinal }} \dot{Q}_{\text {loss }} d t \\
& =\sum_{i=1}^{n t-1} \frac{\left(\dot{Q}_{\text {loss }_{i+1}}+\dot{Q}_{\text {loss }_{i}}\right)}{2} \mathrm{dt}
\end{aligned}
$$

A similar computation is performed for qloss and q_hloss, and these two values are written to the permanent record.

Also in this routine, the exit temperatures are scanned to determine at what time the exit temperature first exceeded $105^{\circ} \mathrm{F}$. If the exit temperature reaches $105^{\circ} \mathrm{F}$, then the time to reach is written out, otherwise a message indicating that the exit temperature never reached $105^{\circ} \mathrm{F}$ is written.

\section{PipeAll::PipeAllTrans(CMatrix \& Told)}

This is a major routine in the program which calls many subroutines. The objective of the routine is to assemble the finite element equations needed and to compute the temperatures everywhere for the current time step. The overall program flow is shown in a flowchart in Fig. G.2. Each of the major routines called by PipeAllTrans() and actions will be described below. 


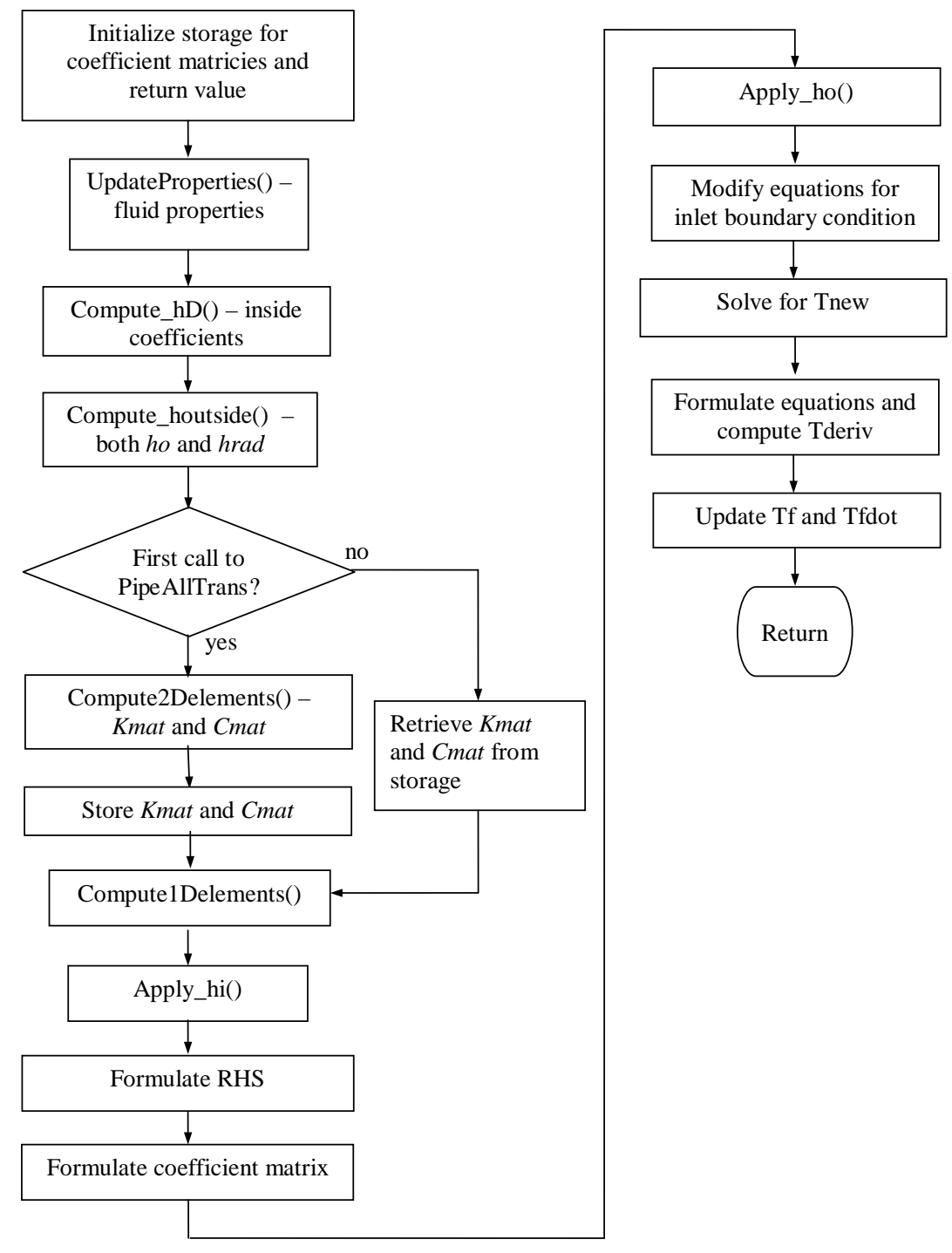

Fig. G.2. Program flow in routine PipeAllTrans.

\section{PipeAll::UpdateProperties(CMatrix \& Told)}

This routine updates the fluid temperature $T f$, which is the average temperature of the fluid in each fluid element. It also computes the average outside surface temperature, Toutside, for each outside element. Based on the values Tf, the properties of the fluid rhof, $c p f$, muf, $k f$, Prf are evaluated using routine WaterProperties().

\section{PipeAll::Compute_hD(CMatrix \& Told)}

This routine computes the heat transfer coefficient on the inside of the pipe. If the mass flow rate is not zero, then a conventional correlation for steady turbulent flow in a pipe is used to compute this heat transfer coefficient: 


$$
\mathrm{Nu}_{D}=\frac{(f / 8)\left(\mathrm{Re}_{D}-1000\right) \operatorname{Pr}}{1+12.7(f / 8)^{1 / 2}\left(\operatorname{Pr}^{2 / 3}-1\right)}
$$

For a zero flow rate, an equivalent convection coefficient must be computed to account for the conduction resistance of the fluid. This was done by using an analytical solution for transient radial heat conduction in a solid cylinder subjected to a step increase in surface temperature as a model. It was found from this analytical model that the "heat transfer coefficient", defined as the heat flux at the outer edge of the cylinder divided by the difference between the surface temperature and the volume averaged temperature inside the cylinder, was dependent only on this difference in temperature. A polynomial curve fit correlation representing this was determined as

$$
\begin{aligned}
\mathrm{Nu}_{D} & =2.9892 \mathrm{E}+06(\Delta T)^{8}-4.6524 \mathrm{E}+06(\Delta T)^{7}+2.9096 \mathrm{E}+06(\Delta T)^{6}-0.93157 \mathrm{E}+06(\Delta T)^{5} \\
& +1.6132 \mathrm{E}+05(\Delta T)^{4}-1.4528 \mathrm{E}+04(\Delta T)^{3}+5.9960 \mathrm{E}+02(\Delta T)^{2} \\
& -7.9867 \mathrm{E}+00(\Delta T)+5.7870 \mathrm{E}+00
\end{aligned}
$$

Here $\Delta T$ is the dimensionless temperature difference, $\Delta T=(T f-T$ wall $) / T$ wall. This correlation is valid for $0.0<\Delta T<0.475$.

\section{PipeAll::Compute_houtside( void )}

This routine computes both heat transfer coefficients on the outside of the pipe: one due to convection and one due to radiation.

The external convection coefficient depend on the external velocity, Vout, which was read from input. If Vout is zero, then free (natural) convection occurs, and a correlation for free convection from horizontal cylinders is used to compute $\mathrm{Nu}_{D}$ :

$$
\overline{N u}_{D}=\left\{0.60+\frac{0.387 R a_{D}^{1 / 6}}{\left[1+(0.559 / \mathrm{Pr})^{9 / 16}\right]^{8 / 27}}\right\}^{2}
$$

If Vout is not zero, then a forced convection coefficient is computed for the outside of a cylinder from

$$
\overline{N u}_{D}=\left\{0.60+\frac{0.387 R a_{D}^{1 / 6}}{\left[1+(0.559 / \mathrm{Pr})^{9 / 16}\right]^{8 / 27}}\right\}^{2}
$$

For the radiation transfer, a radiation coefficient is computed from

$$
h_{\text {rad }}=\varepsilon \sigma\left(T_{\text {sur }}^{2}+T_{\infty}^{2}\right)\left(T_{\text {sur }}+T_{\infty}\right)
$$

\section{PipeAll::Compute2Delements( CMatrix \& Kmat, CMatrix \& Cmat)}

This routine computes the conduction matrix Kmat and the capacitance matrix Cmat for the axisymmetric shell elements in the pipe, insulation, and any surrounding material (SOIL or ATTIC keywords). The theory behind this was revealed previously in the "Development" section. 
Since these computations are time-consuming, and they do not change with time (constant properties are used in the pipe and surroundings), some economy is achieved by calling this routine only once for each segment. The computed values of Kmat and Cmat are stored and later recalled, rather than recomputed.

\section{PipeAll::Compute1Delements( CMatrix \& Kmat, CMatrix \& Cmat)}

This routine is called for each iteration and each time step, since the values in the conductivity matrix Kmat and the capacitance matrix Cmat depend on the fluid temperatures. The finite element theory behind this routine was revealed previously in the "Development" section.

In this routine, a collection of constant "helper" matricies $k h, k c$, and $k m$, are used to compute the elemental matricies $K h, K c$, and $K m$. The latter are derived from the former by multiplying by element-dependent thermophysical properties. These latter matricies are assembled into the global matricies Kmat and Cmat using knowledge of the connectivity (arrangement of the nodes and elements).

The $K h$ portion of the element equation connects the fluid to the pipe wall [Eq. (15f) from the "Development" section]. Knowledge of the pipe wall node numbers is used to insert terms from $K h$ into the appropriate locations of the global Kmat matrix.

\section{PipeAll::Apply_hi( CMatrix \& Kmat )}

This routine applies the effect of the heat transfer coefficient $h D$ between the 1-D fluid elements and the 2-D pipe elements. These are Eqs. (24c) and (24d) from the development. The first portion is the direct contribution to the stiffness matrix Kmat and is assembled in the usual manner based on the connectivity of the nodes. The second portion (Eq. 24d) originally was a load vector contribution due to known adjacent fluid temperatures. Since these fluid temperatures are now unknowns, these terms are inserted into the appropriate places (based on the connectivity of the fluid elements) in the stiffness matrix Kmat.

\section{Solve for Tnew}

This is simply an implementation of the Eq. (11) from the "Development" section. Since the $R$ terms are, at the moment, zero, the vector on the right side of the equation, $b$, is set to ( $(1 . / d t) C m a t-$ (1-theta)Kmat )Told. The coefficient matrix from Eq. (11), Coef, is set to (theta)(Kmat) + Cmat(1./dt).

Next the boundary conditions are applied to the system. The function Apply_ho() adds terms to Coef and $b$ to reflect the heat transfer coefficient boundary condition on the outside of the pipe assembly. Eqs. (24e) and (24f) from the "Development" describe these modifications. Both portions represent an addition to the existing values in the matrix.

The final condition is the specification of the inlet boundary condition. The class variable Tin has been set to either the supply temperature (for segment 1 ) or the exit temperature from the previous segment (for any other segment) in the SetupForTime() routine. The Coef matrix is modified to reflect the necessary condition $T_{1}=$ Tin.

The matrix system is solved using a Gaussian Elimination scheme for banded matricies. 


\section{Solve for Tderiv}

The rate of change of temperature is needed for the fluid elements in order to perform the energy balance and compute the rate of increase of energy in the system. This can be computed directly from the finite element equations [see Eq. (15) in the "Development" section, for example]. The time derivatives are found by solving Coef*Tderiv $=-\mathrm{Kmat}^{*}$ Tnew, but the first equation in this system is modified to reflect the fact that the inlet temperature is fixed (at each time).

\section{Update Tf and Tfdot}

Before returning from PipeAllTrans(), the current values of the fluid temperature $T f$ and its derivative Tfdot are computed. These values are for each fluid element, and are determined as the average temperature or derivative in the element. 


\section{Appendix H. HWDS VALIDATION AND IMPROVEMENT}

This appendix contains the report produced by Dr. Keith Woodbury, detailing the experimental validation and improvements made to the calculational program. 



\title{
HWDS Validation and Improvement
}

\author{
Final Report
}

Submitted to:

Oak Ridge National Laboratory

Building Technology Center

\author{
Submitted by: \\ Keith A. Woodbury \\ Woodbury Engineering Consultants
}

May 7, 2007

$\mathrm{H}-3$ 



\section{Synopsis}

The Hot Water Distribution Simulator (HWDS) was developed by Woodbury Engineering under contract to Oak Ridge National Laboratory in 2003. This simulator is evaluated in this report by comparing results from HWDS to experimental data collected by Applied Energy Technology (AET) [1-2]. This report also details modifications to the HWDS.

Over 130 cases for piping systems in still air were compared, including bare copper pipe in two different diameters (1/2" and 3/4"), copper pipe in two diameters with two different insulation thicknesses, and PEX-AL-PEX piping with and without backing insulation. From these $130+$ tests over a broad range of operating conditions, it can be concluded, with $95 \%$ confidence, that the average steady-state RMS error between the HWDS-computed and the measured temperature along the pipe is $0.539+/-0.071 \mathrm{~F}$. Thus, for piping systems in air environments, the steady-state temperature along the pipe can be predicted using HWDS within about one-half of one degree Fahrenheit.

Only 21 cases of piping systems buried in damp sand were available. The 21 cases available considered 3/4" copper pipe both bare and covered with 3/4" of insulation. A major unknown in the analysis of these cases was the thermal properties of the damp sand.

Reasonable values, consistent with available information and the data, were assumed for thermal conductivity and specific heat for the sand. For bare buried pipe, it was found that varying values of interfacial heat transfer coefficient were necessary to best fit the available data. That is, the thermal properties of the sand in the region around the bare pipe were not constant from test-to-test. For buried pipe covered in insulation the effect is negligible and this was not the case. For the 21 cases, it can be stated, with 95\% confidence, that the average steady-state error between the HWDS and the measured data is $0.694+/-0.091 \mathrm{~F}$. That is, the HWDS predicts the steady distribution along the pipe within about seven-tenths of a degree Fahrenheit.

\section{Introduction}

The subcontractor, Woodbury Engineering Consultants, was engaged to provide verification, modification, and possible enhancement of the ORNL Hot Water Distribution Simulator. The following tasks were specifically assigned:

Task 1. ORNL-HWDS Model Verification: This task involves performing comparison of experimental data to computations from the ORNL-HWDS model. Required finetuning of the model will be accomplished based on the experimental data Experimental data will be gathered from laboratory and/or field studies on hot water distribution systems. These data, consisting of time versus temperature at different axial locations, will be used to modify the conventional correlations to bring the model into agreement with the experiments as follows:

Case A. Still Air Systems: Existing and additional data provided to ORNL from Laboratory tests for flow of hot water through copper, CPVC and/or PEX systems in still air will be used to tune the model. For this case there are heat transfer coefficients on both the inside and outside of the pipe that are determined via correlations. Both these correlations may need to be adjusted to bring the model predictions into the best possible agreement with the experimental values. Most of this experimental data will be provided by ORNL at the start of the project. Any remaining data will be provided by March 1, 2006. 
Case B. Below Slab Systems: New data will be provided to ORNL for piping systems imbedded in sand (moist and dry). For this case, an additional parameter for the interfacial heat transfer coefficient on the outside of the pipe should be incorporated into the model. With appropriate data from experiments, and using procedures developed in Case A, this parameter will be implemented. This data will be provided by ORNL by March 6, 2006.

Task 2. ORNL-HWD Modeling Enhancements: In this task, the model will be expanded to cover a broader range of operating conditions, provided adequate data are made available. The model will be extended to allow for analysis of very low flow conditions (resulting in laminar or stratified flow). Finally, if suitable data in developing regions of pipe are available, the model will be extended to analyze these developing flow situations applicable to piping segments with short lengths. These extensions will be incorporated by implementing appropriate correlations and program logic to select these automatically based on user input. These model augmentations will be validated concurrently using experimental data use in Cases $A$ and $B$.

Task 3. Consultation: This task will provide consultation as needed in support of ORNL's efforts beyond that provided in Tasks $1 \& 2$. It could include such items as a peer review of the reports to California Energy Commission, or adjustment to the model as a result of ORNL's activities on the project. The seller may charge up to 20 hours for this task.

Tasks $1 \mathrm{a}$ and $1 \mathrm{~b}$ have been completed using data supplied by ORNL from AET [1-2]. Comparisons of HWDS results to data are provided in this report. Some modifications to the HWDS have been made and are detailed in the appendix.

Task 2 was contingent on availability of suitable data from very low flow conditions and detailed data in very short runs of pipes. These data were not delivered and no further discussions regarding this task were held between ORNL and the subcontractor. This task cannot be completed under this work agreement.

The subcontractor is available for consultation under Task 3 following submission of this report.

\section{General Modeling Issues}

The HWDS program has been generalized to allow for segments of different pipe materials and also to input a draw schedule. Appendix $E$ gives details of this generalization. The new input format and output format are independently selectable using compile switches VERSION_20_INPUT and VERSION_20_OUTPUT, so it is possible to run cases using the old style input file. For all of the analyses in this report, the VERSION_20_INPUT switch was \#undef'd and the VERSION_20_OUTPUT was \#define'd. The VERSION_20_OUTPUT includes the computation of the UA/L for each segment in the pipe.

\section{UA/L from Data}

The overall heat transfer coefficient per unit length was determined from the raw experimental data provided by AET for each of the 130+ cases considered. This value was obtained by considering an exponential curve fit of the dimensionless steady temperature 
distribution along the pipe. For water flowing in a pipe exchanging heat with a constant temperature source, the temperature distribution of the water along the pipe is [3].

$$
\frac{T_{s}-T(x)}{T_{s}-T_{i n}}=\exp \left(\frac{U A / L}{\dot{m} c_{p}} x\right)
$$

Case120: s100604a 3/4" CU BARE 1.8107 GPM

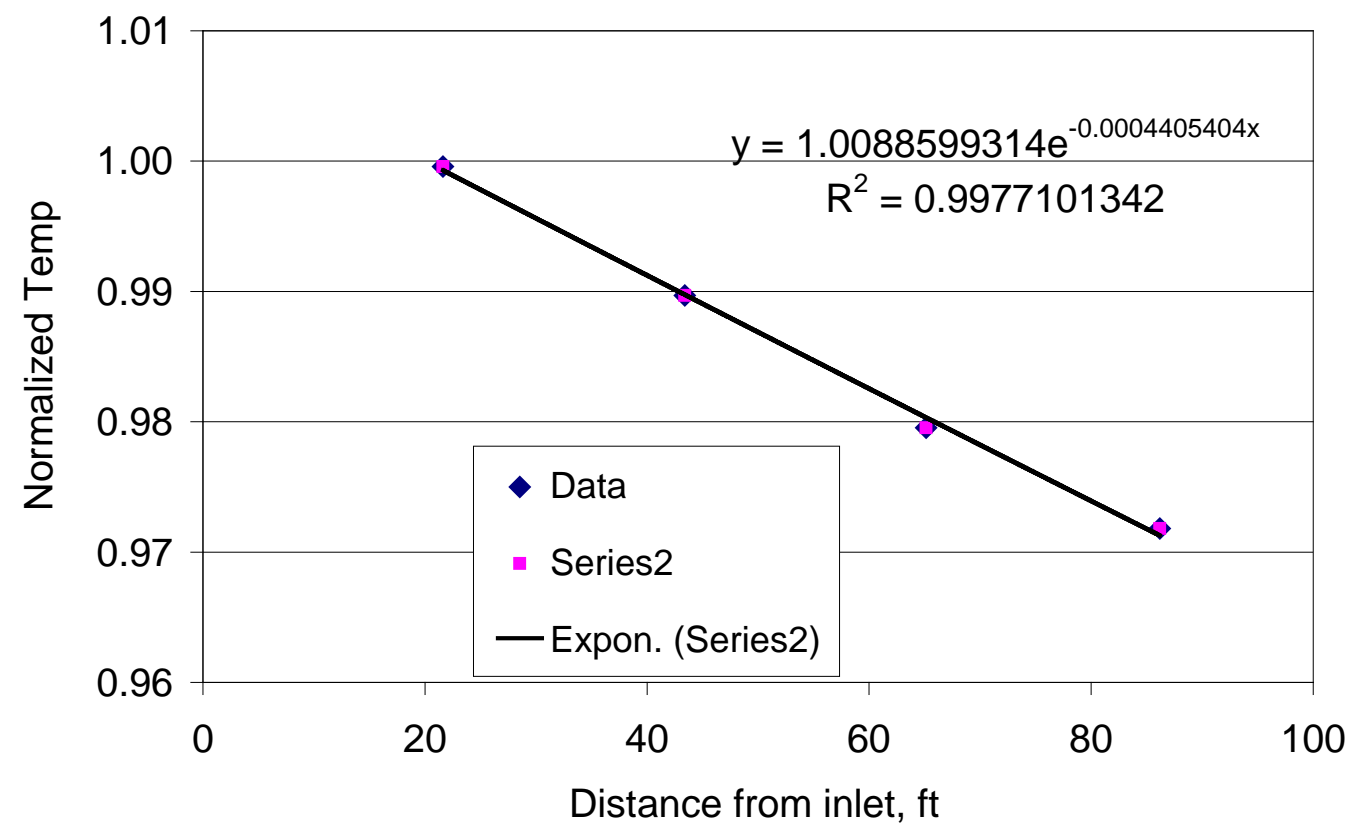

Fig. 2 Dimensionless temperature distribution and curve fit used to determine UA/L

In Eq. (1), $T_{\mathrm{s}}$ is the constant temperature environment and $T_{\text {in }}$ is the inlet temperature of the water. By plotting the steady dimensionless temperature distribution from Eq. (1) for each case and performing an exponential curve fit, the value of the coefficient in the exponent in Eq. (1) can be determined. Fig. 1 shows an example of such a plot. Based on knowledge of the flow rate and the specific heat of the flowing water, the UA/L quantity can be determined.

\section{UA/L from HWDS}

The HWDS program was modified to compute the overall heat transfer coefficient per unit length UA/L. This quantity is computed and output from the program if VERSION_20_OUTPUT is \#define'd at compile time. This value is computed as the sum of thermal resistances of the inside heat transfer coefficient, the pipe wall, the insulation (if present), and the external heat transfer coefficient and radiation. Specifically: 


$$
\begin{aligned}
R_{\text {inside }} & =\frac{1}{h_{i} 2 \pi r_{i}} \\
R_{\text {pipe }} & =\frac{\ln \left(r_{o} / r_{i}\right)}{2 \pi k_{\text {pipe }}} \\
R_{\text {ins }} & =\frac{\ln \left(\left(r_{o}+\Delta r_{\text {ins }} / r_{o}\right)\right.}{2 \pi k_{\text {ins }}} \\
R_{\text {outside }} & =\frac{1}{\left(h_{o}+h_{\text {rad }}\right) 2 \pi\left(r_{o}+\Delta r_{\text {ins }}\right)} \\
(\mathrm{UA} / L)_{\text {HWDS }} & =\frac{1}{R_{\text {inside }}+R_{\text {pipe }}+R_{\text {ins }}+R_{\text {outpuside }}}
\end{aligned}
$$

\section{Thermal Properties}

Model results can only be as good as data that are put into them. For piping systems with copper pipe in air, all thermal properties are well-known. However, for PEX-AL-PEX piping the thermal properties of the pipe are not well-established, and for piping systems buried in damp sand virtually no information was available for thermal properties of this environment. Properties of copper pipe and insulation used in the simulations of pipe systems in air are listed in Table 1.

Table 2 Properties of copper pipe and insulation

\begin{tabular}{|l|c|c|}
\hline & Copper Pipe & Insulation \\
\hline Thermal Conductivity, Btu/hr/ft/F & 227. & 0.0217 \\
\hline Density, lb/ft ${ }^{3}$ & 556. & 0.48 \\
\hline Specific Heat, Btu/lb/F & 0.092 & 0.58 \\
\hline Surface emissivity & 0.072 & 0.90 \\
\hline
\end{tabular}

For piping systems with PEX-AL-PEX piping, there is some uncertainty in the thermal properties. PEX-AL-PEX is a three layered flexible piping that consists of an inner layer of PEX tubing, a middle layer of aluminum, and an outer layer of PEX. The properties assumed for these base components are listed in Table 2.

Table 3 Properties of PEX pipe and Aluminum

\begin{tabular}{|l|c|c|}
\hline & PEX & Aluminum \\
\hline Thermal Conductivity, Btu/hr/ft/F & 0.28 & 100 \\
\hline Density, lb/ft ${ }^{3}$ & 59.3 & 169. \\
\hline Specific Heat, Btu/lb/F & 0.54 & 0.214 \\
\hline
\end{tabular}

\section{Thermal conductivity of pipe wall}

HWDS is configured to have a single (isotropic) value for thermal properties. Calculation of the effective density and specific heat for the layered construction is straightforward, since these quantities are directly related to mass. However, the thermal conductivity is not massdependent, and the effective thermal conductivity will be different in the directions parallel and perpendicular to the pipe centerline. Since the primary direction for heat loss is 
perpendicular to the pipe wall, an effective conductivity based on the series resistance of the three layers was used:

$$
\begin{aligned}
& k_{r}=\frac{\ln \left(r_{o} / r_{i}\right)}{2 \pi\left(R_{P E X 1}+R_{A L}+R_{P E X 2}\right)} \\
& R_{P E X 1}=\frac{\ln \left(\left(r_{i}+\Delta r_{P E X i}\right) / r_{i}\right)}{2 \pi k_{P E X}} \\
& R_{A L}=\frac{\ln \left(\left(r_{o}-\Delta r_{P E X o}\right) /\left(r_{i}+\Delta r_{P E X i}\right)\right)}{2 \pi k_{A L}} \\
& R_{P E X 2}=\frac{\ln \left(r_{o} /\left(r_{o}-\Delta r_{P E X o}\right)\right)}{2 \pi k_{P E X}}
\end{aligned}
$$

The effective value of $k_{r}$ was computed to be $k_{r}=0.311 \mathrm{Btu} / \mathrm{hr} / \mathrm{ft} / \mathrm{F}$. All the results shown in Appendix B for PEX-AL-PEX piping use this isotropic value of $k_{r}$.

\section{Non-isotropic Conductivity}

To investigate the possible importance of non-isotropic thermal conductivity, especially for the PEX-AL-PEX construction, a more general model for conductivity was incorporated into HWDS. This feature is selectable at compile-time by defining the variable USE_COMPOSITE_K and allows for specification of two values of thermal conductivity: one in the radial direction, and one in the axial direction. The effective value of the conductivity in the axial direction was computed by considering the parallel paths for heat flow through the PEX and the aluminum, and the value of $k_{x}=10.92 \mathrm{Btu} / \mathrm{hr} / \mathrm{ft} / \mathrm{F}$ was determined. Several cases were computed using the non-isotropic thermal conductivity, and no perceptible difference was noted in the graphical comparisons. Therefore, reported results are for isotropic value of conductivity with $k=k_{r}=0.311 \mathrm{Btu} / \mathrm{hr} / \mathrm{ft} / \mathrm{F}$.

\section{Task 1a-Piping systems in air}

Over 130 test cases were extracted from raw data files supplied by the Applied Energy Technology (AET) laboratory [2] for piping systems in still air. The tests were conducted during 2004 using 1/2" and 3/4" copper piping and PEX-AL-PEX flexible piping. Both types of pipe were tested bare in air and with expanded foam insulation covering the pipe.

\section{Quantitative comparison-pipes in air}

The table in Appendix $A$ lists all the cases considered and references each case back to a data file from AET. The first eight columns in the table give identifying information for the case, and the other columns give results obtained for each case. The column labeled "UA/L S. S." was generated from the AET data only and is the steady-state value of UA/L. The column labeled "HWDS UA/L" is the value computed by the simulation program and reported if VERSION_20_OUTPUT is \#define'd.

The RMS error between the UA/L determined from the data and that computed by HWDS is $0.020 \mathrm{Btu} / \mathrm{hr} / \mathrm{ft} / \mathrm{F}$. The grand average of all the UA/L values determined from the data is $0.3253 \mathrm{Btu} / \mathrm{hr} / \mathrm{ft} / \mathrm{F}$, so the RMS error represents an average error of $6.3 \%$. That is, the HWDS computes the UA/L within about $6 \%$ of values determined from experimental data. 
The next six columns in the table of Appendix A contain the differences between the measured steady-state temperature [2] and that computed by HWDS at corresponding axial distances from the pipe entrance. The last column contains the RMS error (average difference without regard to sign) of these six "errors". The average RMS error for the 130+ cases is, with $95 \%$ confidence, $0.5239+/-0.0706 \mathrm{~F}$.

\section{Qualitative comparison-pipes in air}

Appendix B contains graphical comparisons of the results computed from HWDS and those from AET data for over 130 cases. Qualitatively the comparison is quite good, especially for copper pipes. As mentioned previously, for copper pipes the thermal properties of the pipe AND backing foam material are well-documented, and hence good comparisons with data are possible.

Overall, the qualitative comparison between the data and HWDS is not as good for PEX-ALPEX as for copper piping. In particular, there is an unexplained trend for the measured temperature to rise earlier than the HWDS prediction (for example, cases 91, 96, and 97 are typical of many) and most notably at the last two stations. This rise begins earlier than the "wave" of hot water could have traveled down the pipe, which suggests that there may be significant axial mixing induced into the flow. This could occur, for example, due to rotational flow through sweeping bends in the pipe where measurements were taken. See Fig. E-1 of reference 2 and note measurement point in foreground.

\section{Task 1b-Buried Piping Systems}

A significant component of this subcontract is the comparison of HWDS computations to experimental data from piping systems buried in damp sand. The Applied Energy Technology laboratory conducted a series of experiments on copper pipe buried in sand in 2006 [1] and sent a number of large raw data files to the subcontractor. These data files contained 21 cases for comparison: ten cases with bare pipe and 11 cases with insulationcovered pipe.

\section{Thermal Properties of Wet Sand}

A challenge to the analyst for buried pipe systems is to provide appropriate values of thermal properties for the surrounding medium. In particular, if the surrounding medium is moisture-bearing, the properties, especially thermal conductivity, are difficult to predict.

Reference 1 reported the test medium consisted of "25.5 tons of washed sand" in a container $24 \mathrm{ft}$ long, $8 \mathrm{ft}$ wide, and filled to a depth of approximately 30 inches. Based on this information, an average bulk density of $106.25 \mathrm{lb} / \mathrm{ft}^{3}$ was determined. Reference 3 gives the density of "sand" as $94.6 \mathrm{lb} / \mathrm{ft}^{3}$. Taking this as density of dry sand, a moisture content of about $7.5 \%$ for the moist sand was inferred. Using this value of moisture content, and properties of "sand" from Reference [3], the mass averaged specific heat of the moist sand was determined to be $0.168 \mathrm{Btu} / \mathrm{lb} / \mathrm{F}$. Table 3 summarizes these properties. 
Table 4 Properties of Dry Sand [3] and Moist Sand

\begin{tabular}{|l|c|c|}
\hline & "Sand" [3] & Moist Sand \\
\hline Moisture content, Vol \% & 0.0 & 7.5 \\
\hline Density, Ib/ft & 94.6 & 106.3 \\
\hline Specific Heat, Btu/lb/F & 0.191 & 0.168 \\
\hline
\end{tabular}

There is no model to predict thermal conductivity of moist sand based on moisture content, and an ad-hoc procedure was used here to determine a value of bulk thermal conductivity consistent with the data. References 3,4 , and 5 provide reasonable bracketing values. Reference 3 gives the conductivity of dry sand as $0.156 \mathrm{Btu} / \mathrm{hr} / \mathrm{ft} / \mathrm{F}$. Reference 4 reports on foundry sand, including "green" sand with moisture content, giving a value of about 0.235 $\mathrm{Btu} / \mathrm{hr} / \mathrm{ft} / \mathrm{F}$. Reference 5 reports on in-situ measurement for soil, which perhaps has a higher density than sand used here, but reported values for conductivity are $1.36 \mathrm{Btu} / \mathrm{hr} / \mathrm{ft} / \mathrm{F}$.

To determine the appropriate bulk thermal conductivity of the moist sand, the first case recorded by $A E T$ in the data files sent to the subcontractor was analyzed many times with different values of thermal conductivity. A value of $k_{\text {sand }}=0.50 \mathrm{Btu} / \mathrm{hr} / \mathrm{ft} / \mathrm{F}$ was found to match the data well (see Case S001 in Appendix D).

\section{Probable Transport Mechanism}

The ability of HWDS to accurately predict the temperature histories in the pipe for Case S001 (Appendix D) from a purely conductive model with constant thermal properties of the sand seems to discount the possibility of significant energy transfer through moisture evaporation and migration. However, in subsequent calculations it was found that different values of thermal conductivity, consistent with lower moisture content, were necessary in order to match data from tests conducted after an initial test in a sequence. This will be explained further below. However, it appears that the purely conductive model is appropriate for computing the heat loss from and resulting temperature distribution along a buried pipe.

\section{Gap Conductance}

To account for imperfect contact between the pipe and the insulation, or the pipe and surrounding sand, or between the pipe/insulation and insulation/sand, an interfacial heat transfer coefficient (gap conductance) was implemented. Details of the gap element development can be found in the appendix. The use of gap elements can be implemented at compile time by \#define-ing USE_GAP_ELEMENTS. The gap elements proved useful in improving the agreement between the model and data, especially for the low-flow cases.

\section{Quantitative Comparison}

Appendix $\mathrm{C}$ gives a table with results from HWDS for the 21 cases of buried pipe. UA/L was computed from the data, as described previously, and also by HWDS. The RMS error between the two methods was found to be $0.0217 \mathrm{Btu} / \mathrm{hr} / \mathrm{ft} / \mathrm{F}$. The grand average of the $\mathrm{UA} / \mathrm{L}$ determined from the data for these 21 cases was $0.4571 \mathrm{Btu} / \mathrm{hr} / \mathrm{ft} / \mathrm{F}$, resulting in an average difference of $4.7 \%$. It should be noted that the HWDS prediction is consistently overestimated, since it includes a conduction resistance for the surrounding sand (in a form similar to Eq. (2b) or (2c)) based on the physical burial depth. In all cases, the effective penetration depth of the "heat wave" into the sand is quite small, as observed by thermocouple measurements in the sand in reference 1 . Thus, the resistance to heat flow is 
overestimated by the HWDS prediction, but still the calculation produces a value within $4 \%$ (on average).

A quasi-steady state is reached for each simulation, generally within 200 seconds. The temperatures measured and those predicted by HWDS were compared for this "SteadyState" condition. Over the 21 tests available, it can be stated, with 95\% confidence, that the RMS error between the measured and computed "steady-state" temperatures is $0.694 \mathrm{~F}+/$ $0.091 \mathrm{~F}$. That is, the steady temperature profiles can be computed within about 0.75 degrees Fahrenheit.

\section{Thermal conductivity and importance of contact conductance}

As mentioned earlier, it was noted that, for multiple tests conducted in a given day, that acceptable agreement with later tests was not possible..

In all of the cases for bare copper pipe, it was necessary to include the effect of a gap conductance between the pipe and the surrounding sand. For the cases considered, gap contact conductances of $10<h_{\text {gap }}<60 \mathrm{Btu} / \mathrm{hr} / \mathrm{ft}^{2} / \mathrm{F}$ were necessary to match the data. The nature of this variation is consistent with a lower contact conductance in later tests on the same day. The values of $h_{\text {gap }}$ used on each simulation are listed in the table in Appendix C.

\section{Qualitative Comparison}

Appendix $\mathrm{D}$ gives the resulting graphical comparison between the measured temperature data and corresponding HWDS computations. The first ten cases are for bare pipe and, as mentioned, the contact conductance and/or the thermal conductivity of the medium required adjustment in order to obtain a favorable comparison. For the last eleven cases, which had a layer of insulation surrounding the pipe, the effect of the contact conductance is overshadowed by the presence of the insulation. Reasonable comparisons with the data were possible using the assumed $k_{\text {sand }}=0.50 \mathrm{Btu} / \mathrm{hr} / \mathrm{ft} / \mathrm{F}$ and no contact conductance between the insulation and sand.

\section{Conclusions}

- HWDS gives very good agreement with measured experimental data for temperature distributions in piping systems in air

- HWDS gives good agreement with measured experimental data for temperature distributions in piping systems buried under sand.

- Thermal conditions in the vicinity of the buried pipe change with time for repeated draws. After a long time the conditions appear to recover to the initial state.

- Knowledge of thermal properties of surrounding sand is necessary to get good results for buried pipe systems. This data is not readily available in handbooks.

\section{References}

[ 1 ] Hiller, C. C., "Bare vs Insulated Buried Pipe Test Interim Report," LBNL SUBCONTRACT NO. 6803876, Sept., 2006

[ 2 ] Hiller, C. C., "Hot Water Distribution System Research - Phase I Final Report," Final Report, Contract No. C-03-23A California Institute for Energy Efficiency, May, 2005 
[ 3 ] Incropera, F., DeWitt, D., Bergman, T., and Lavine, A., Fundamentals of Heat and Mass Transfer, $6^{\text {th }}$ Edition, John Wiley, 2005.

[ 4 ] Midea, T., and Shah, V., "Mold Material Thermophysical Data," AFS Transactions, 02-080, 2002

[ 5 ] Shonder, J. A., and Beck, J. V., "A New Method to Determine the Thermal Properties of Soil Formations from In Situ Field Tests," ORNL/TM-2000/97 

APPENDICES

H-15 

Appendix A. TABULAR RESULTS FROM HWDS FOR PIPES IN AIR

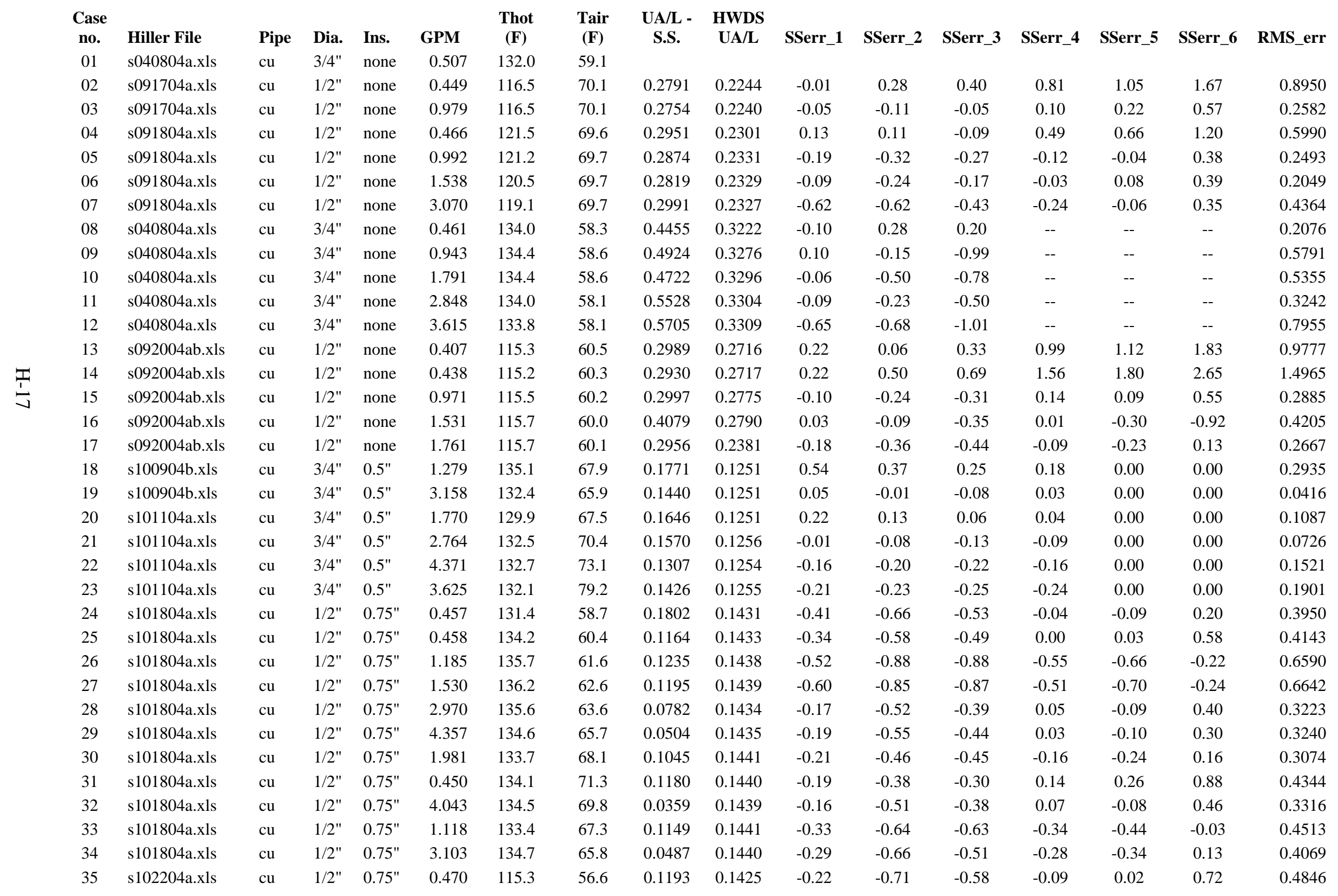




\begin{tabular}{|c|c|c|c|c|c|c|c|c|c|c|c|c|c|c|c|c|}
\hline $\begin{array}{c}\text { Case } \\
\text { no. }\end{array}$ & Hiller File & Pipe & Dia. & Ins. & GPM & $\begin{array}{l}\text { Thot } \\
\text { (F) }\end{array}$ & $\begin{array}{l}\text { Tair } \\
\text { (F) }\end{array}$ & $\begin{array}{c}\text { UA/L - } \\
\text { S.S. }\end{array}$ & $\begin{array}{l}\text { HWDS } \\
\text { UA/L }\end{array}$ & SSerr_1 & SSerr_2 & SSerr_3 & SSerr_4 & SSerr_5 & SSerr_6 & RMS_err \\
\hline 36 & s102204a.xls & $\mathrm{cu}$ & $1 / 2^{\prime \prime}$ & $0.75 "$ & 0.442 & 115.5 & 56.6 & 0.1159 & 0.1424 & -0.12 & -0.17 & -0.08 & 0.31 & 0.46 & 1.04 & 0.4894 \\
\hline 37 & s102204a.xls & $\mathrm{cu}$ & $1 / 2 "$ & $0.75 "$ & 0.442 & 115.5 & 56.6 & 0.1159 & 0.1424 & -0.12 & -0.17 & -0.08 & 0.31 & 0.46 & 1.04 & 0.4894 \\
\hline 38 & s102204a.xls & $\mathrm{cu}$ & $1 / 2 "$ & $0.75 "$ & 0.457 & 116.0 & 56.2 & 0.1159 & 0.1424 & -0.11 & -0.14 & -0.06 & 0.36 & 0.53 & 1.22 & 0.5695 \\
\hline 39 & s102204a.xls & $\mathrm{cu}$ & $1 / 2^{\prime \prime}$ & $0.75 "$ & 4.037 & 115.7 & 56.5 & 0.0826 & 0.1422 & -0.12 & -0.22 & -0.18 & 0.01 & -0.08 & 0.23 & 0.1602 \\
\hline 40 & s102204a.xls & $\mathrm{cu}$ & $1 / 2 "$ & $0.75 "$ & 1.068 & 116.3 & 57.7 & 0.1171 & 0.1428 & 0.05 & 0.02 & 0.11 & 0.36 & 0.39 & 0.83 & 0.4061 \\
\hline 41 & s102204a.xls & $\mathrm{cu}$ & $1 / 2 "$ & $0.75 "$ & 1.126 & 116.3 & 59.1 & 0.1250 & 0.1428 & -0.13 & -0.24 & -0.16 & -0.06 & -0.06 & 0.15 & 0.1479 \\
\hline 42 & s102204a.xls & $\mathrm{cu}$ & $1 / 2 "$ & $0.75 "$ & 1.601 & 115.8 & 61.6 & 0.1186 & 0.1431 & 0.01 & -0.08 & -0.02 & 0.09 & 0.10 & 0.29 & 0.1344 \\
\hline 43 & s102204a.xls & $\mathrm{cu}$ & $1 / 2^{\prime \prime}$ & $0.75 "$ & 1.582 & 116.2 & 63.1 & 0.1150 & 0.1431 & -0.12 & -0.24 & -0.18 & -0.06 & -0.08 & 0.14 & 0.1489 \\
\hline 44 & s102204a.xls & $\mathrm{cu}$ & $1 / 2^{\prime \prime}$ & $0.75 "$ & 2.986 & 115.4 & 66.1 & 0.0919 & 0.1434 & -0.91 & -0.93 & -0.79 & -0.65 & -0.53 & -0.21 & 0.7159 \\
\hline 45 & s102204a.xls & $\mathrm{cu}$ & $1 / 2^{\prime \prime}$ & $0.75 "$ & 3.004 & 116.7 & 68.7 & 0.1105 & 0.1427 & -0.06 & -0.15 & -0.08 & 0.00 & 0.00 & 0.19 & 0.1055 \\
\hline 46 & s111504a.xls & $\mathrm{cu}$ & $1 / 2^{\prime \prime}$ & $0.50^{\prime \prime}$ & 3.146 & 116.5 & 56.8 & 0.1097 & 0.1251 & -0.19 & -0.34 & -0.24 & -0.08 & -0.10 & 0.23 & 0.2155 \\
\hline 47 & s111504a.xls & $\mathrm{cu}$ & $1 / 2^{\prime \prime}$ & $0.50 "$ & 0.498 & 116.1 & 56.7 & 0.1319 & 0.1250 & -0.09 & -0.11 & 0.22 & 0.74 & 1.09 & 1.86 & 0.9364 \\
\hline 48 & s111504a.xls & $\mathrm{cu}$ & $1 / 2^{\prime \prime}$ & $0.50 "$ & 1.226 & 116.5 & 56.7 & 0.1348 & 0.1252 & -0.12 & -0.25 & -0.16 & 0.13 & 0.20 & 0.73 & 0.3402 \\
\hline 49 & s111504a.xls & $\mathrm{cu}$ & $1 / 2^{\prime \prime}$ & $0.50^{\prime \prime}$ & 2.076 & 116.5 & 56.5 & 0.1167 & 0.1251 & -0.06 & -0.15 & -0.03 & 0.25 & 0.38 & 0.89 & 0.4153 \\
\hline 50 & s111504a.xls & $\mathrm{cu}$ & $1 / 2^{\prime \prime}$ & $0.50^{\prime \prime}$ & 3.795 & 116.3 & 56.4 & 0.1051 & 0.1252 & -0.15 & -0.29 & -0.17 & 0.02 & -0.02 & 0.29 & 0.1921 \\
\hline 51 & s111504a.xls & $\mathrm{cu}$ & $1 / 2^{\prime \prime}$ & $0.50^{\prime \prime}$ & 2.897 & 116.5 & 56.3 & 0.1161 & 0.1252 & -0.05 & -0.18 & -0.07 & 0.15 & 0.13 & 0.44 & 0.2133 \\
\hline 52 & s111504a.xls & $\mathrm{cu}$ & $1 / 2^{\prime \prime}$ & $0.50 "$ & 4.172 & 132.4 & 56.0 & 0.0966 & 0.1255 & -0.17 & -0.36 & -0.20 & -0.03 & -0.07 & 0.25 & 0.2107 \\
\hline 53 & s111504a.xls & $\mathrm{cu}$ & $1 / 2^{\prime \prime}$ & $0.50 "$ & 0.455 & 133.5 & 56.2 & 0.1450 & 0.1253 & -0.15 & -0.26 & -0.03 & 0.44 & 0.63 & 0.84 & 0.4822 \\
\hline 54 & s111504a.xls & $\mathrm{cu}$ & $1 / 2^{\prime \prime}$ & $0.50 "$ & 3.019 & 134.7 & 56.2 & 0.1590 & 0.1255 & -0.23 & -0.42 & -0.41 & -0.23 & -0.29 & -0.09 & 0.2990 \\
\hline 55 & s111504a.xls & $\mathrm{cu}$ & $1 / 2^{\prime \prime}$ & $0.50^{\prime \prime}$ & 2.092 & 134.6 & 56.2 & 0.2043 & 0.1255 & -0.26 & -0.37 & -0.36 & -0.30 & -0.46 & -0.48 & 0.3804 \\
\hline 56 & s111504a.xls & $\mathrm{cu}$ & $1 / 2^{\prime \prime}$ & $0.50^{\prime \prime}$ & 1.165 & 135.2 & 57.4 & 0.1463 & 0.1256 & -0.37 & -0.50 & -0.35 & -0.08 & -0.10 & 0.09 & 0.2974 \\
\hline 57 & s111504a.xls & $\mathrm{cu}$ & $1 / 2^{\prime \prime}$ & $0.50^{\prime \prime}$ & 2.937 & 136.0 & 58.4 & 0.1055 & 0.1256 & -0.38 & -0.46 & -0.36 & -0.08 & -0.09 & 0.24 & 0.3057 \\
\hline 58 & s111504a.xls & $\mathrm{cu}$ & $1 / 2^{\prime \prime}$ & $0.50^{\prime \prime}$ & 0.469 & 135.0 & 59.9 & 0.1305 & 0.1255 & -0.19 & -0.20 & 0.08 & 0.65 & 0.86 & 1.72 & 0.8376 \\
\hline 59 & s111504a.xls & $\mathrm{cu}$ & $1 / 2 "$ & $0.50 "$ & 1.166 & 136.1 & 62.6 & 0.1306 & 0.1258 & -0.47 & -0.62 & -0.37 & -0.11 & -0.01 & 0.55 & 0.4203 \\
\hline 60 & s111504a.xls & $\mathrm{cu}$ & $1 / 2^{\prime \prime}$ & $0.50 "$ & 4.201 & 135.8 & 65.0 & 0.0785 & 0.1259 & -0.36 & -0.42 & -0.33 & -0.07 & -0.08 & 0.18 & 0.2770 \\
\hline 61 & s122004a.xls & PAX & 3/4" & none & 0.914 & 115.2 & 47.6 & 0.5083 & 0.5424 & 0.45 & 0.13 & -0.27 & -0.82 & 0.00 & 0.00 & 0.3994 \\
\hline 62 & s122004a.xls & PAX & 3/4" & none & 4.480 & 115.6 & 49.0 & 0.6392 & 0.7279 & 0.87 & 0.75 & 0.68 & 0.16 & 0.00 & 0.00 & 0.5483 \\
\hline 63 & s122004a.xls & PAX & 3/4" & none & 0.488 & 115.1 & 49.3 & 0.5151 & 0.6926 & 0.67 & 0.55 & 0.47 & 0.07 & 0.00 & 0.00 & 0.4028 \\
\hline 64 & s122004a.xls & PAX & 3/4" & none & 3.146 & 116.0 & 50.0 & 0.5150 & 0.7276 & 0.76 & 0.88 & 0.82 & 0.38 & 0.00 & 0.00 & 0.6007 \\
\hline 65 & s122004a.xls & PAX & 3/4" & none & 3.417 & 115.5 & 50.9 & 0.3634 & 0.7266 & 1.11 & 1.24 & 1.40 & 1.17 & 0.00 & 0.00 & 1.0083 \\
\hline 66 & s122004a.xls & PAX & 3/4" & none & 0.899 & 115.1 & 51.5 & 0.5048 & 0.7119 & 0.72 & 0.78 & 0.69 & 0.37 & 0.00 & 0.00 & 0.5378 \\
\hline 67 & s122004a.xls & PAX & 3/4" & none & 1.883 & 115.4 & 52.0 & 0.4982 & 0.7226 & 0.83 & 0.91 & 0.87 & 0.60 & 0.00 & 0.00 & 0.6621 \\
\hline 68 & s122004a.xls & PAX & 3/4" & none & 0.474 & 115.0 & 52.0 & 0.5087 & 0.6913 & 0.40 & 0.33 & 0.23 & 0.05 & 0.00 & 0.00 & 0.2329 \\
\hline 69 & s122004a.xls & PAX & 3/4" & none & 0.943 & 115.4 & 51.9 & 0.5204 & 0.7133 & 0.66 & 0.74 & 0.53 & 0.21 & 0.00 & 0.00 & 0.4669 \\
\hline 70 & s122004a.xls & PAX & 3/4" & none & 4.411 & 115.7 & 52.1 & 0.4606 & 0.7288 & 0.94 & 0.87 & 0.90 & 0.55 & 0.00 & 0.00 & 0.6775 \\
\hline 71 & s122004a.xls & PAX & 3/4" & none & 1.891 & 115.1 & 51.8 & 0.5039 & 0.7219 & 0.88 & 0.95 & 0.90 & 0.79 & 0.00 & 0.00 & 0.7198 \\
\hline 72 & s122004a.xls & PAX & 3/4" & none & 1.829 & 115.6 & 52.1 & 0.4832 & 0.7227 & 0.85 & 0.78 & 0.89 & 0.60 & 0.00 & 0.00 & 0.6443 \\
\hline 73 & s122004a.xls & PAX & 3/4" & none & 3.163 & 113.9 & 53.0 & 0.4395 & 0.7236 & 0.94 & 0.96 & 0.98 & 0.73 & 0.00 & 0.00 & 0.7393 \\
\hline
\end{tabular}




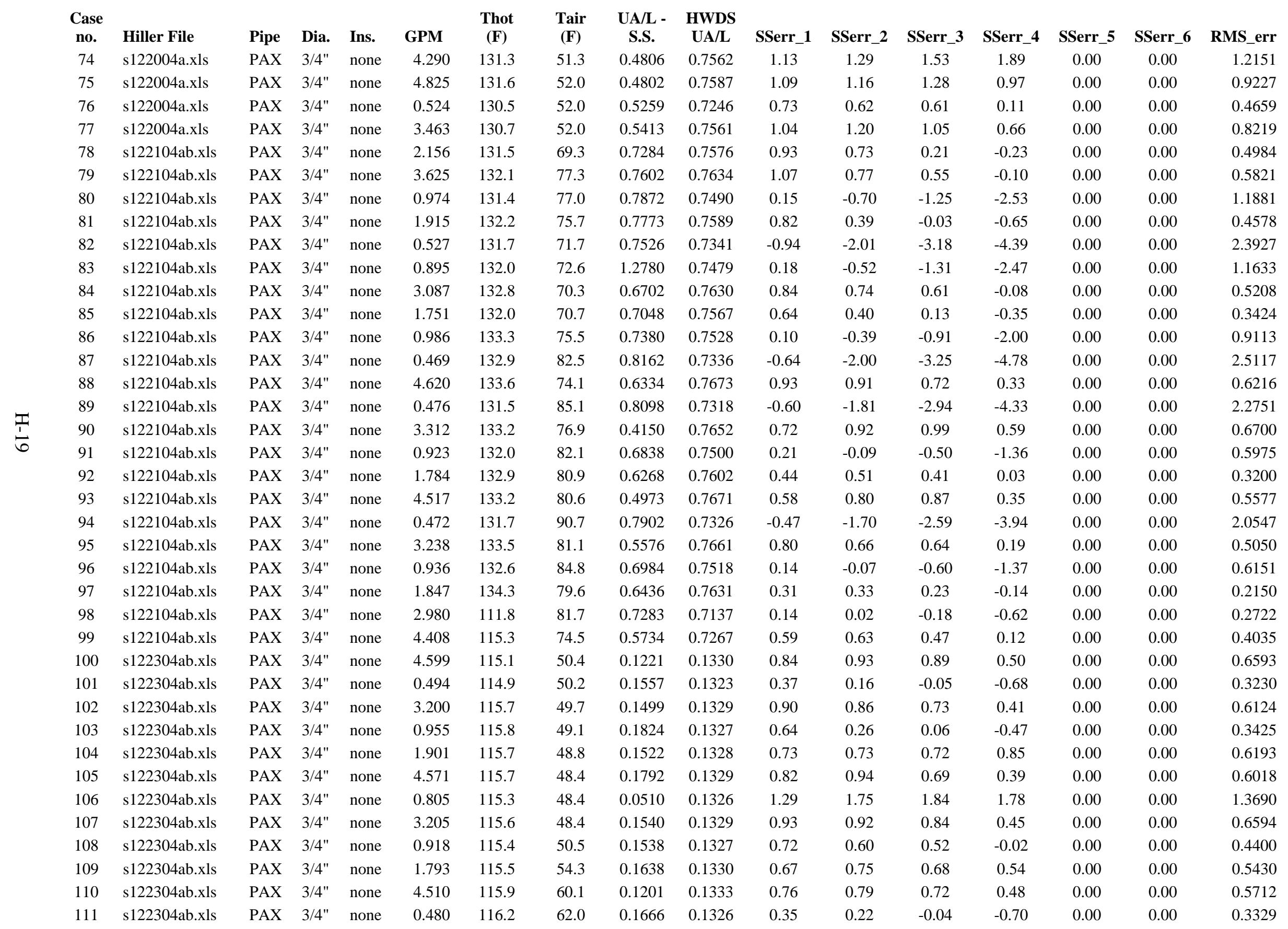




\begin{tabular}{|c|c|}
\hline $\begin{array}{c}\text { Case } \\
\text { no. }\end{array}$ & Hiller File \\
\hline 112 & s122304ab.xls \\
\hline 113 & s122304ab.xls \\
\hline 114 & s122304ab.xls \\
\hline 115 & s122304ab.xls \\
\hline 116 & s070804bc.xls \\
\hline 117 & s070804bc.xls \\
\hline 118 & s070804bc.xls \\
\hline 119 & s070804bc.xls \\
\hline 120 & s100604a.xls \\
\hline 121 & s100604a.xls \\
\hline 122 & s100604a.xls \\
\hline 123 & s100604a.xls \\
\hline 124 & s100604a.xls \\
\hline 125 & s100604a.xls \\
\hline 126 & s100604a.xls \\
\hline 127 & s091704a.xls \\
\hline 128 & s091704a.xls \\
\hline 129 & s091704a.xls \\
\hline 130 & s091704a.xls \\
\hline 131 & s091704a.xls \\
\hline 132 & s111504a.xls \\
\hline 133 & s111504a.xls \\
\hline
\end{tabular}

$\begin{array}{lllcc} & & & & \text { Thot } \\ \text { Pipe } & \text { Dia. } & \text { Ins. } & \text { GPM } & \text { (F) } \\ \text { PAX } & 3 / 4 " & \text { none } & 0.455 & 115.7 \\ \text { PAX } & 3 / 4 " & \text { none } & 3.125 & 116.1 \\ \text { PAX } & 3 / 4 " & \text { none } & 4.456 & 115.8 \\ \text { PAX } & 3 / 4 " & \text { none } & 0.480 & 115.4 \\ \text { cu } & 3 / 4 " & 0.75 " & 0.420 & 114.3 \\ \text { cu } & 3 / 4 " & 0.75 " & 0.996 & 114.8 \\ \text { cu } & 3 / 4 " & 0.75 " & 2.142 & 115.2 \\ \text { cu } & 3 / 4 " & 0.75 " & 1.065 & 134.0 \\ \text { cu } & 3 / 4 " & \text { none } & 1.811 & 132.3 \\ \text { cu } & 3 / 4 " & \text { none } & 2.681 & 133.6 \\ \text { cu } & 3 / 4 " & \text { none } & 4.204 & 133.3 \\ \text { cu } & 3 / 4 " & \text { none } & 2.422 & 132.9 \\ \text { cu } & 3 / 4 " & \text { none } & 3.183 & 133.7 \\ \text { cu } & 3 / 4 " & \text { none } & 1.674 & 133.5 \\ \text { cu } & 3 / 4 " & \text { none } & 0.875 & 132.7 \\ \text { cu } & 1 / 2 " & \text { none } & 0.444 & 116.2 \\ \mathrm{cu} & 1 / 2 " & \text { none } & 1.035 & 116.0 \\ \mathrm{cu} & 1 / 2 " & \text { none } & 1.701 & 116.2 \\ \mathrm{cu} & 1 / 2 " & \text { none } & 2.825 & 116.0 \\ \mathrm{cu} & 1 / 2 " & \text { none } & 0.979 & 115.8 \\ \mathrm{cu} & 1 / 2 " & \text { none } & 2.954 & 133.6 \\ \mathrm{cu} & 1 / 2 " & \text { none } & 1.168 & 134.6\end{array}$

Tair UA/L - HWDS

$\begin{array}{cccccc}\mathbf{( F )} & \text { S.S. } & \text { UA/L } & \text { SSerr_1 } & \text { SSerr_2 } & \text { SSerr_3 } \\ 62.5 & 0.2609 & 0.1326 & 0.25 & 0.11 & -0.30 \\ 63.4 & 0.1739 & 0.1334 & 0.57 & 0.59 & 0.50 \\ 67.2 & 0.1036 & 0.1335 & 0.66 & 0.64 & 0.62 \\ 64.2 & 0.1628 & 0.1327 & 0.63 & 0.25 & -0.10 \\ 65.2 & 0.1596 & 0.1241 & 0.09 & 0.06 & -0.12 \\ 71.3 & 0.1613 & 0.1248 & 0.33 & 0.27 & 0.20 \\ 77.9 & 0.1551 & 0.1252 & 0.34 & 0.34 & 0.32 \\ 83.7 & 0.1670 & 0.1254 & 0.36 & 0.31 & 0.38 \\ 65.2 & 0.3967 & 0.3206 & 0.52 & 0.36 & 0.17 \\ 65.2 & 0.4220 & 0.3227 & 0.59 & 0.43 & 0.29 \\ 65.0 & 0.3816 & 0.3232 & 0.63 & 0.51 & 0.42 \\ 64.9 & 0.3971 & 0.3221 & 0.59 & 0.42 & 0.30 \\ 66.5 & 0.3820 & 0.3217 & 0.68 & 0.54 & 0.46 \\ 68.4 & 0.4071 & 0.3183 & 0.47 & 0.26 & 0.11 \\ 70.2 & 0.3232 & 0.3134 & 0.55 & 0.45 & 0.39 \\ 70.9 & 0.6428 & 0.2247 & -0.35 & -0.54 & -1.16 \\ 70.6 & 0.2889 & 0.2276 & -0.18 & -0.38 & -0.49 \\ 70.4 & 0.2831 & 0.2288 & -0.19 & -0.38 & -0.42 \\ 70.7 & 0.2779 & 0.2288 & -0.24 & -0.29 & -0.22 \\ 73.0 & 0.2808 & 0.2247 & -0.08 & -0.19 & -0.34 \\ 67.4 & 0.1079 & 0.1261 & -0.43 & -0.55 & -0.43 \\ 69.0 & 0.1272 & 0.1261 & -0.10 & -0.38 & -0.32\end{array}$

SSerr_4
-0.38
0.29
0.29
-0.48
-0.32
0.12
0.28
1.02
0.13
0.22
0.49
0.28
0.48
-0.02
0.32
-1.30
-0.58
-0.46
-0.06
-0.39
-0.25
-0.19

$\begin{array}{ccr}\text { SSerr_5 } & \text { SSerr_6 } & \text { RMS_err } \\ 0.00 & 0.00 & 0.2277 \\ 0.00 & 0.00 & 0.4119 \\ 0.00 & 0.00 & 0.4681 \\ 0.00 & 0.00 & 0.3402 \\ 0.00 & 0.00 & 0.1470 \\ 0.00 & 0.00 & 0.1985 \\ 0.00 & 0.00 & 0.2613 \\ 0.00 & 0.00 & 0.4858 \\ 0.00 & 0.00 & 0.2713 \\ 0.00 & 0.00 & 0.3312 \\ 0.00 & 0.00 & 0.4236 \\ 0.00 & 0.00 & 0.3405 \\ 0.00 & 0.00 & 0.4481 \\ 0.00 & 0.00 & 0.2224 \\ 0.00 & 0.00 & 0.3565 \\ -2.19 & -11.85 & \\ -0.65 & -0.61 & 0.5080 \\ -0.51 & -0.37 & 0.4009 \\ -0.01 & 0.23 & 0.2028 \\ -0.47 & -0.54 & 0.3699 \\ -0.29 & -0.03 & 0.3696 \\ -0.28 & 0.01 & 0.2483\end{array}$

$\begin{array}{rr}\text { AVG } & 0.5239 \\ \text { St DEV } & 0.4120 \\ \text { +/- err } & 0.0706\end{array}$




\section{Appendix B. GRAPHICAL COMPARISON OF HWDS RESULTS TO MEASURED DATA FOR PIPE SYSTEMS IN AIR}

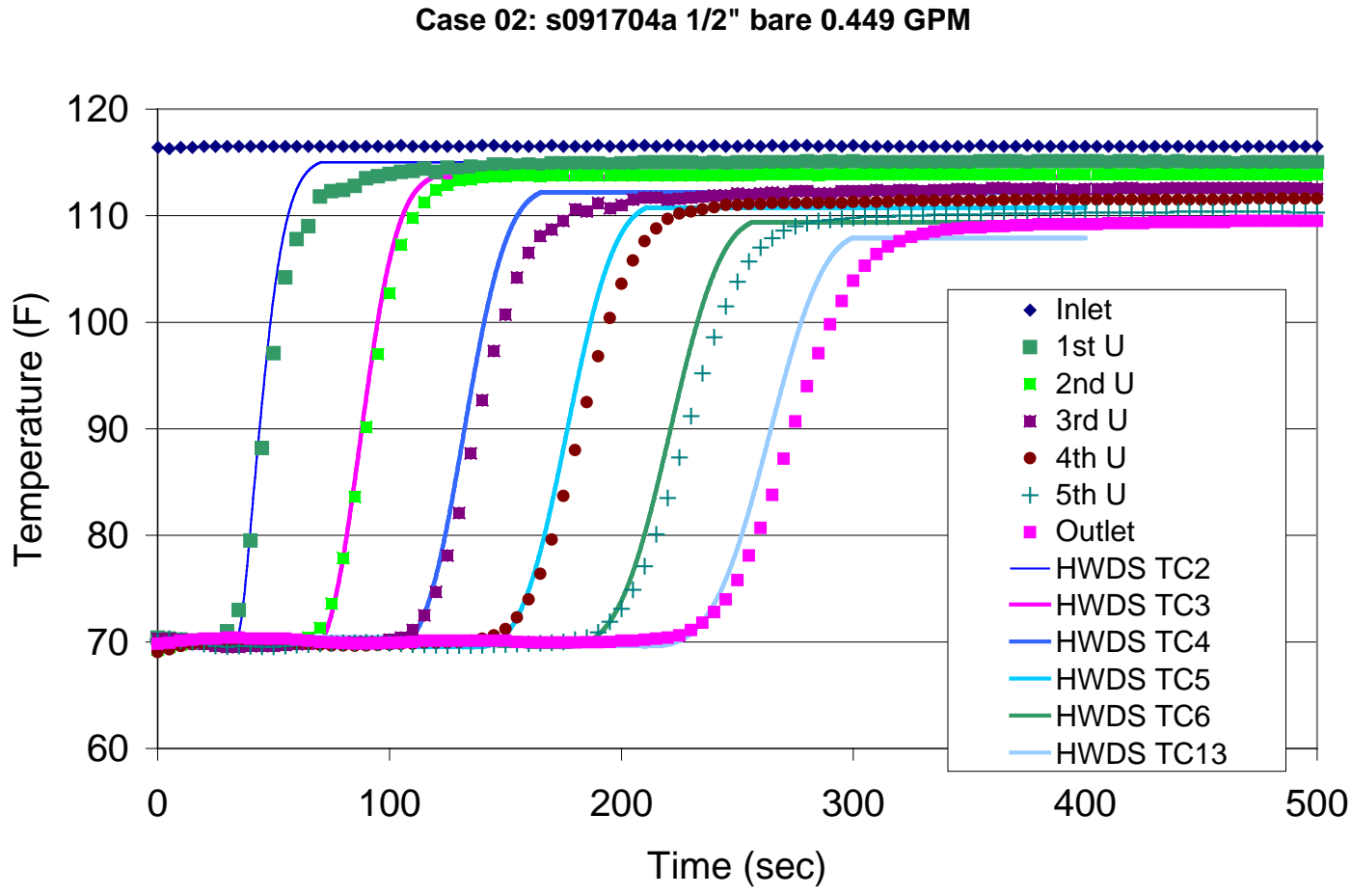

Case03: s091704a 1/2" bare 0.979 GPM

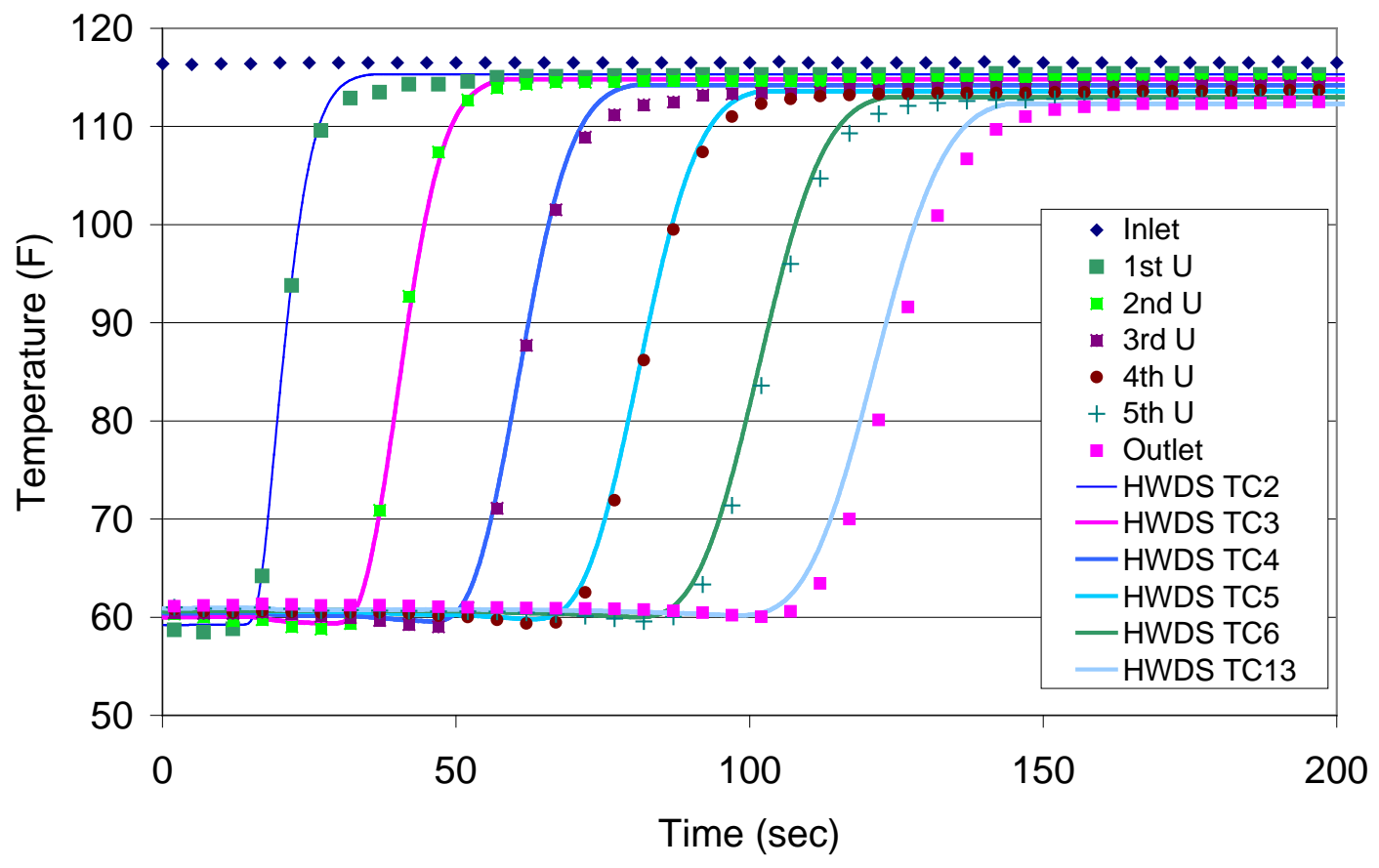




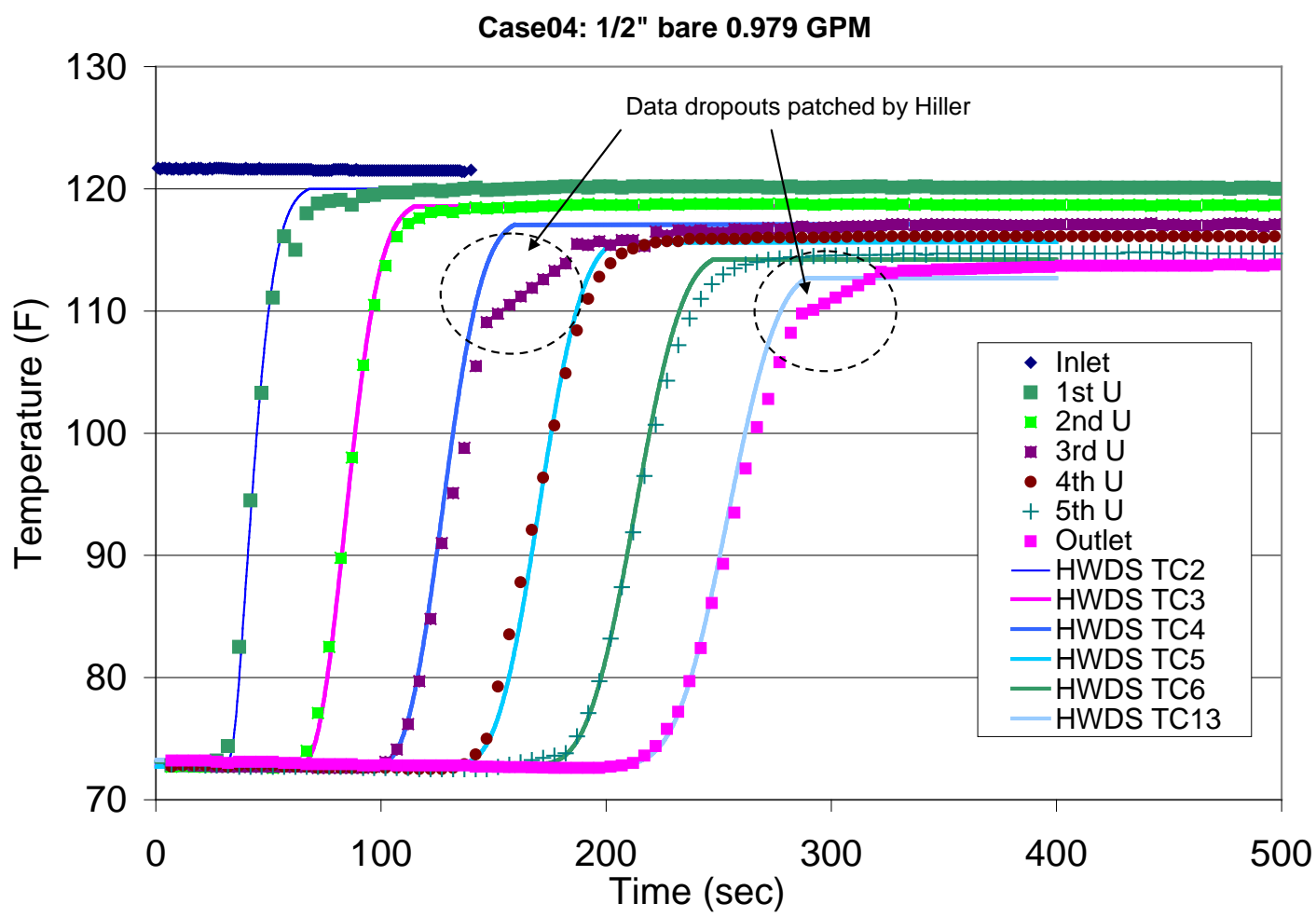

Case05: 1/2" bare 0.992 GPM

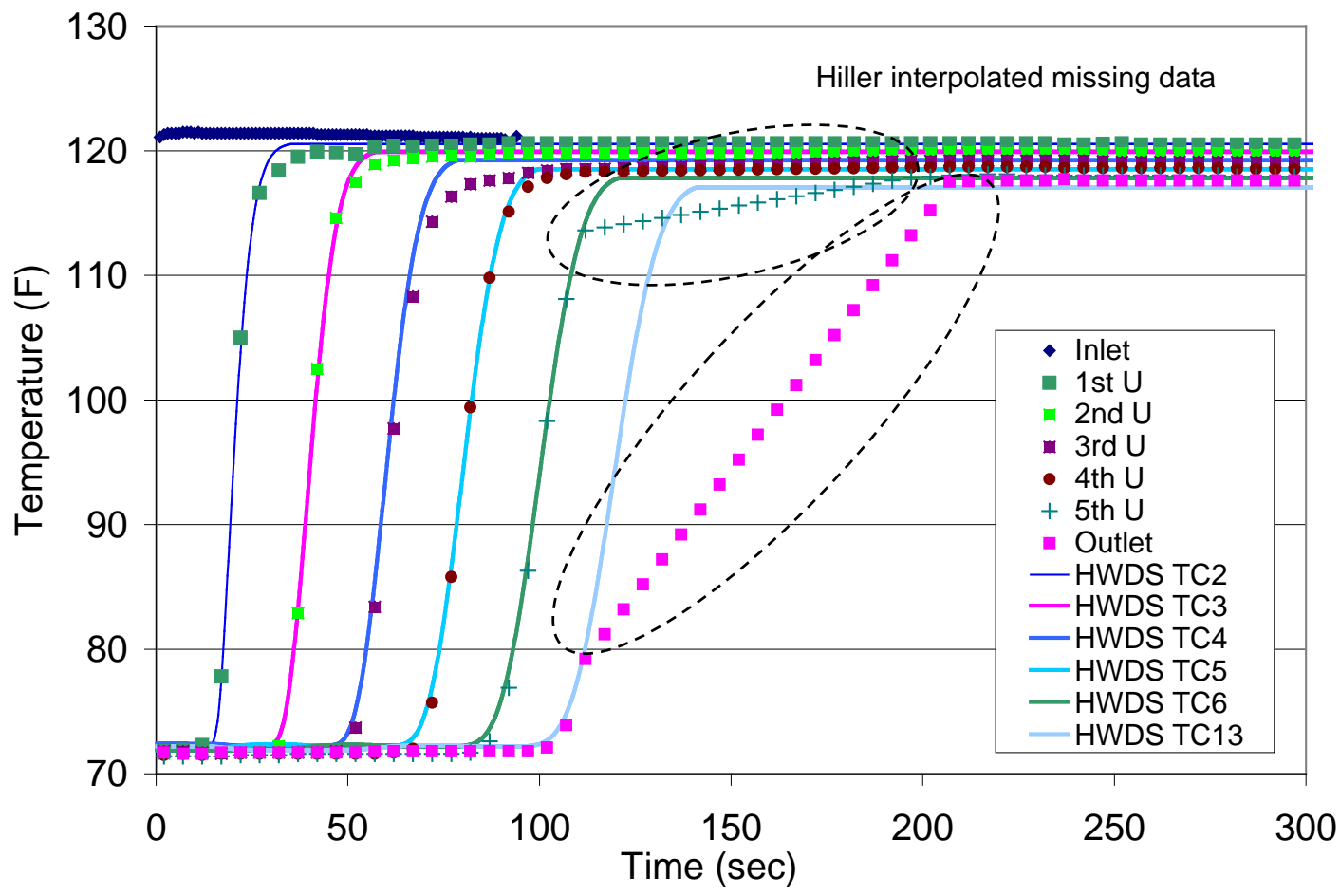



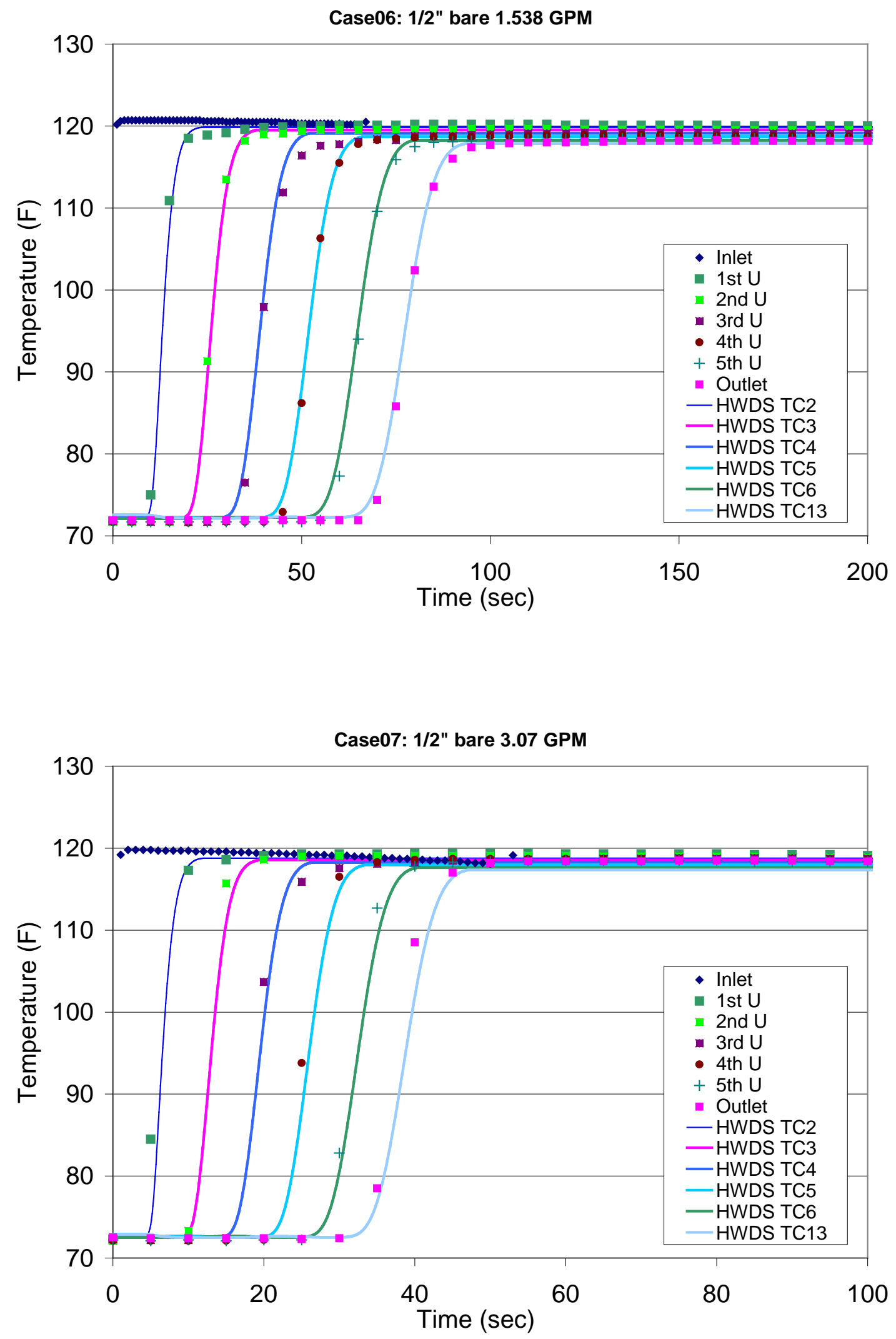
Case09: s040804a 3/4" bare 0.943 GPM

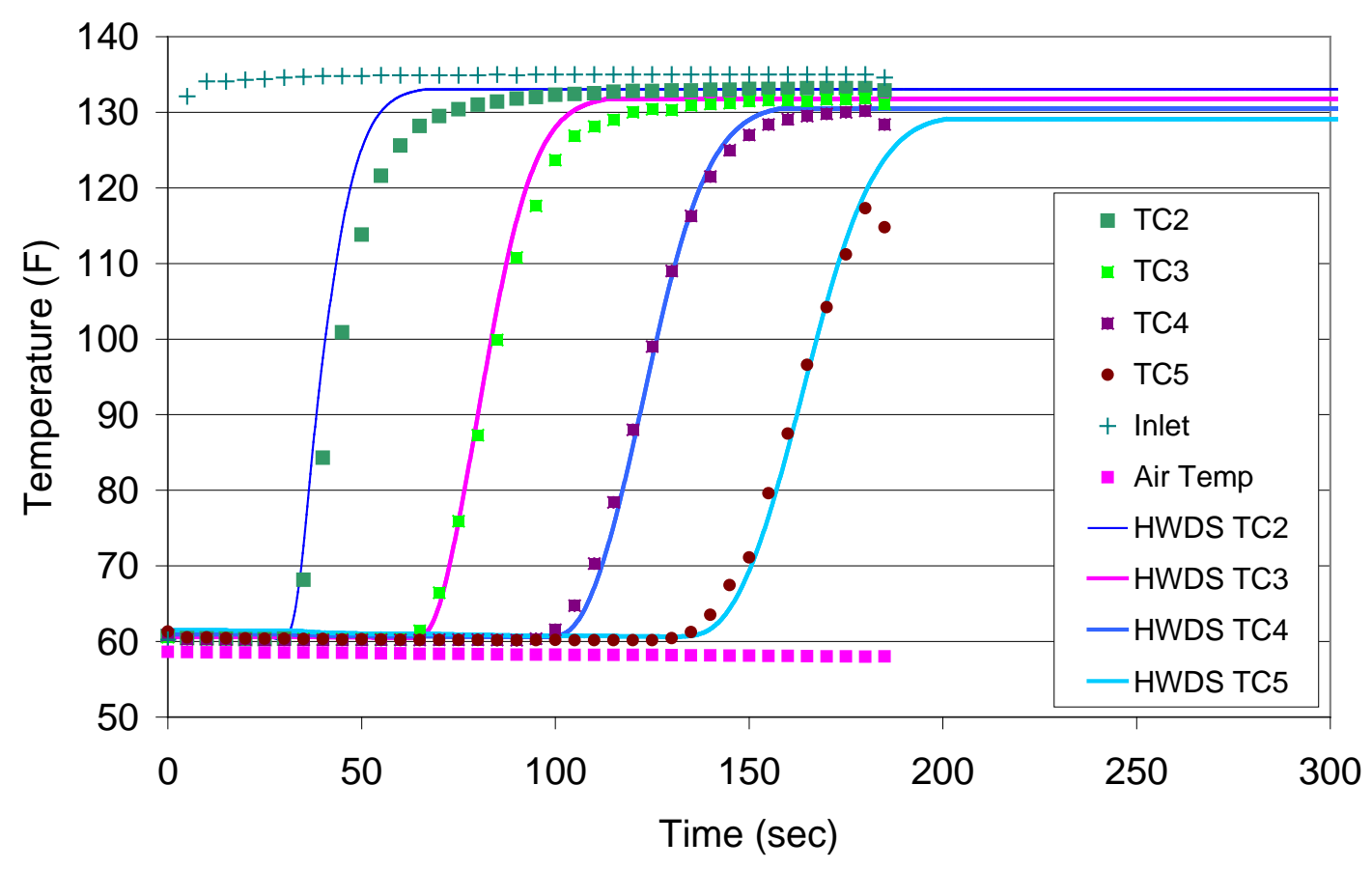

Case10: s040804a 3/4" bare 1.79 GPM

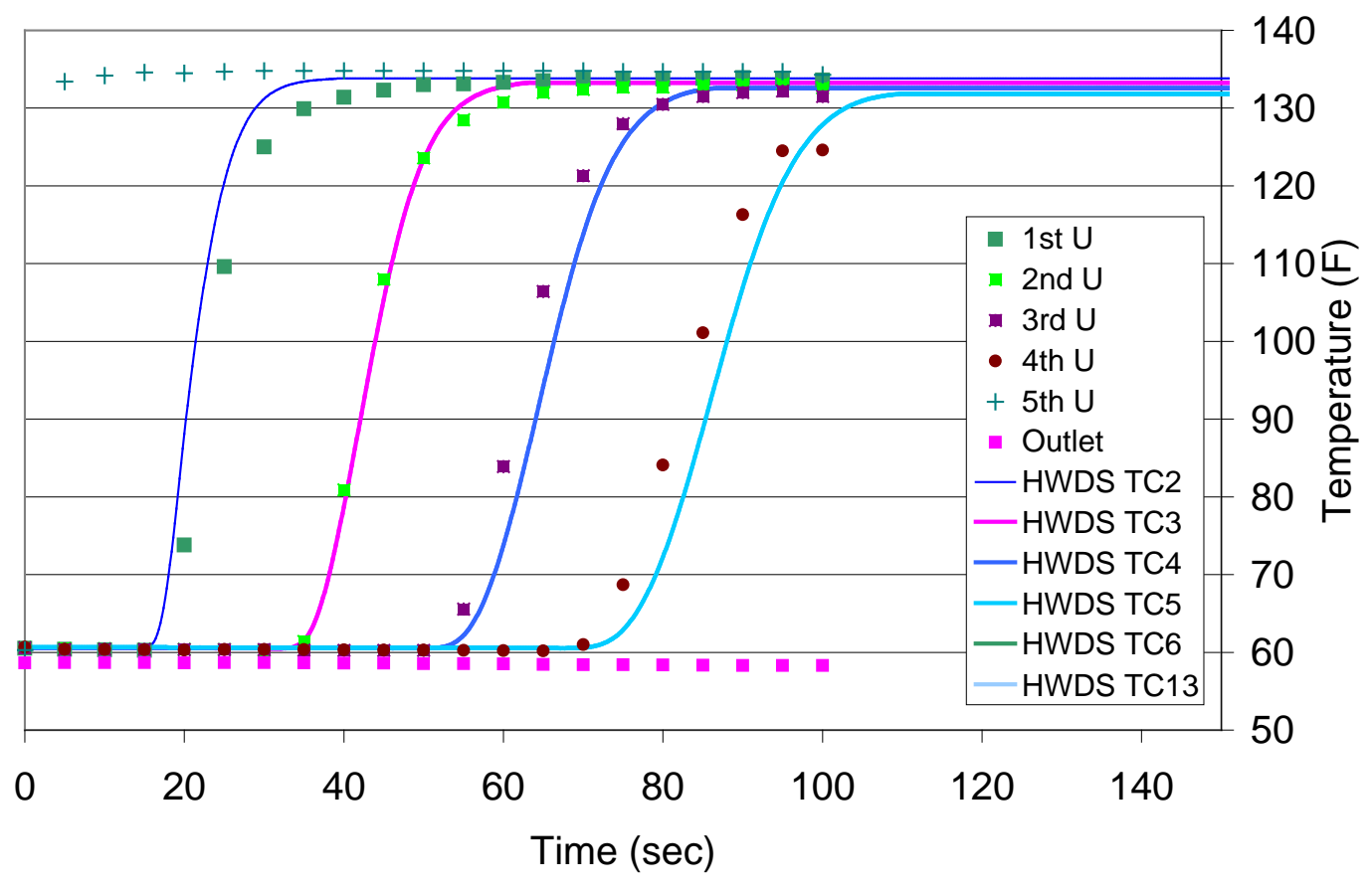


Case11: s040804a 3/4" bare 2.85 GPM

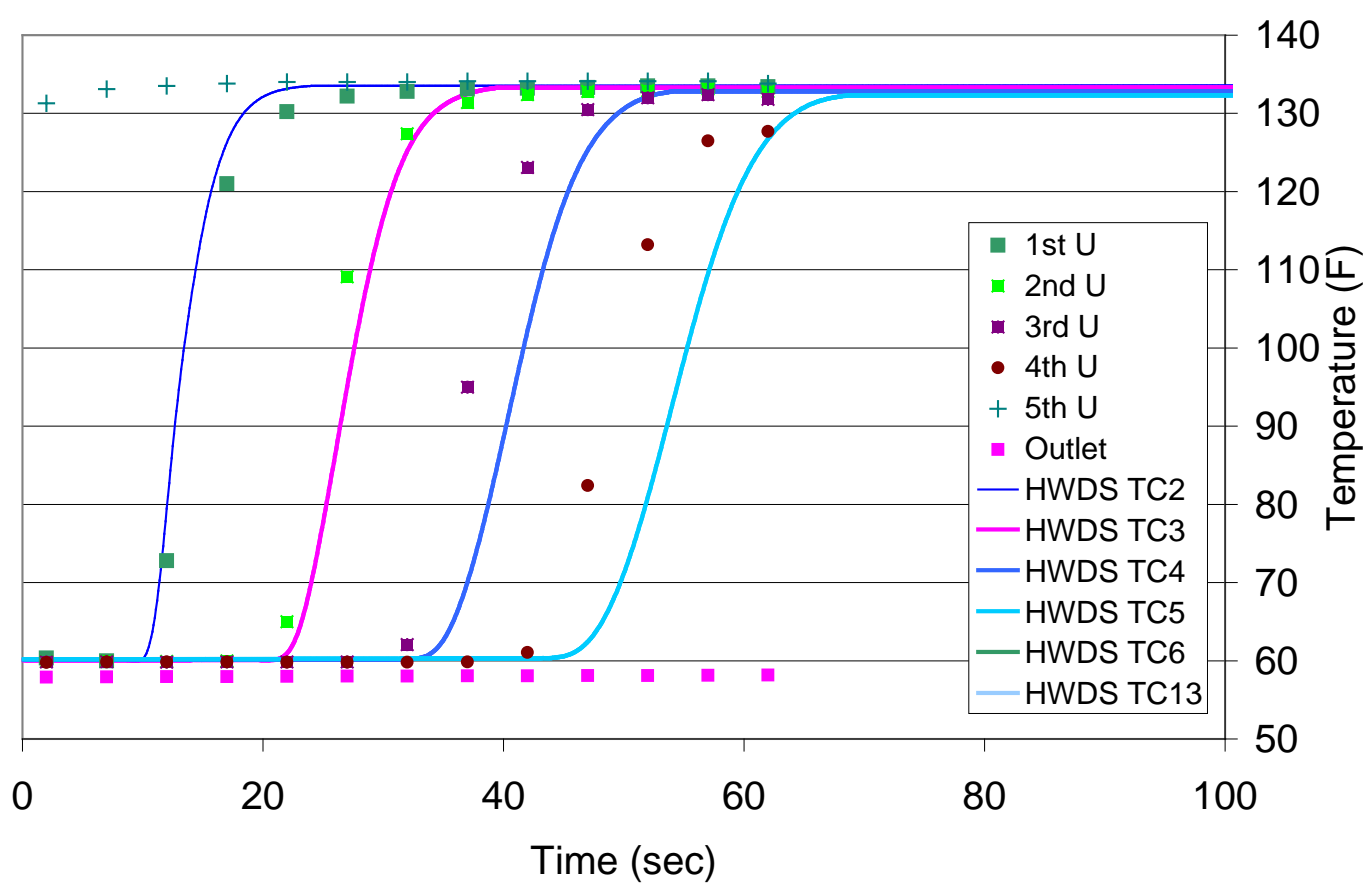

Case12: s040804a 3/4" bare 3.615 GPM

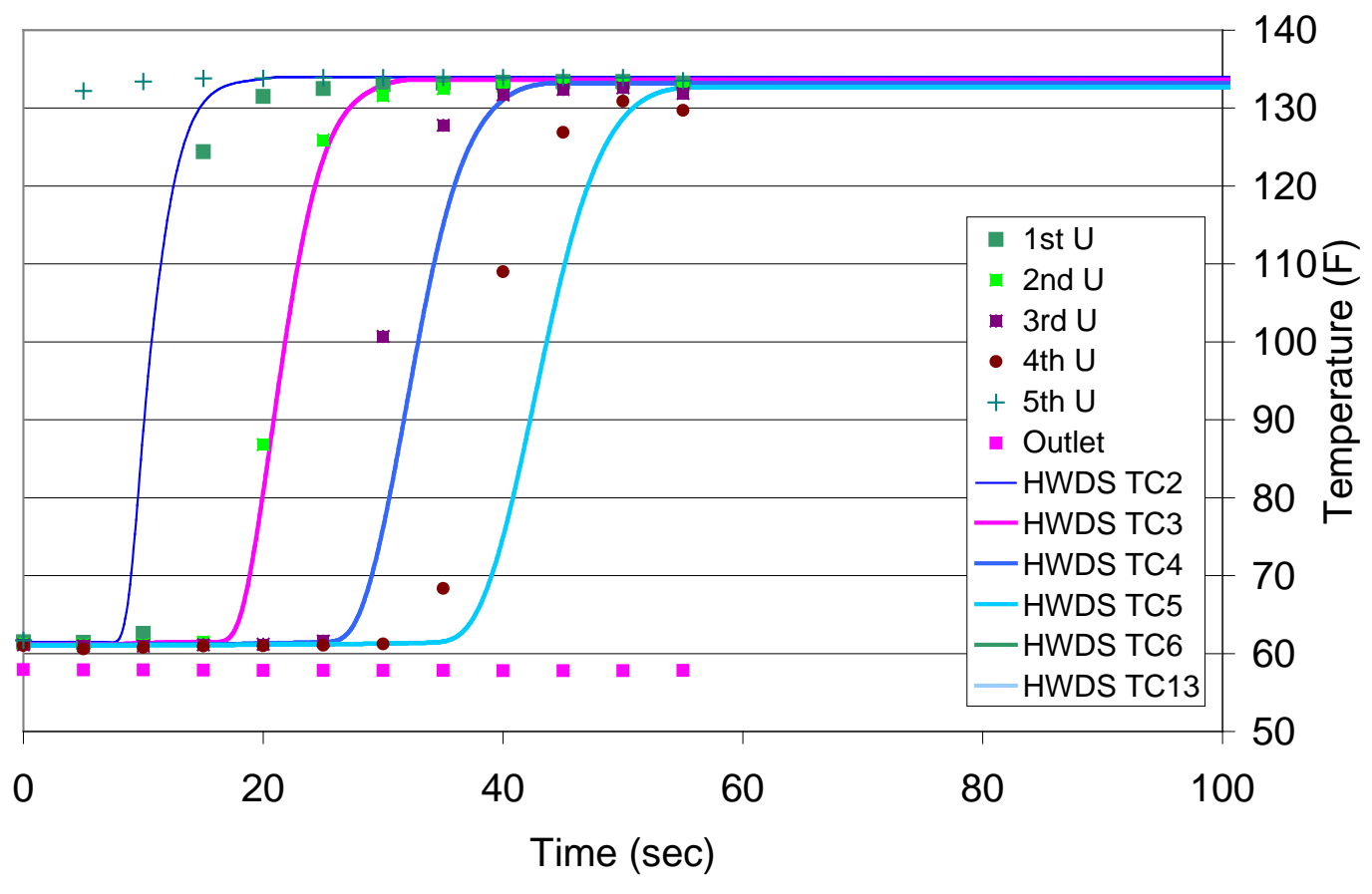




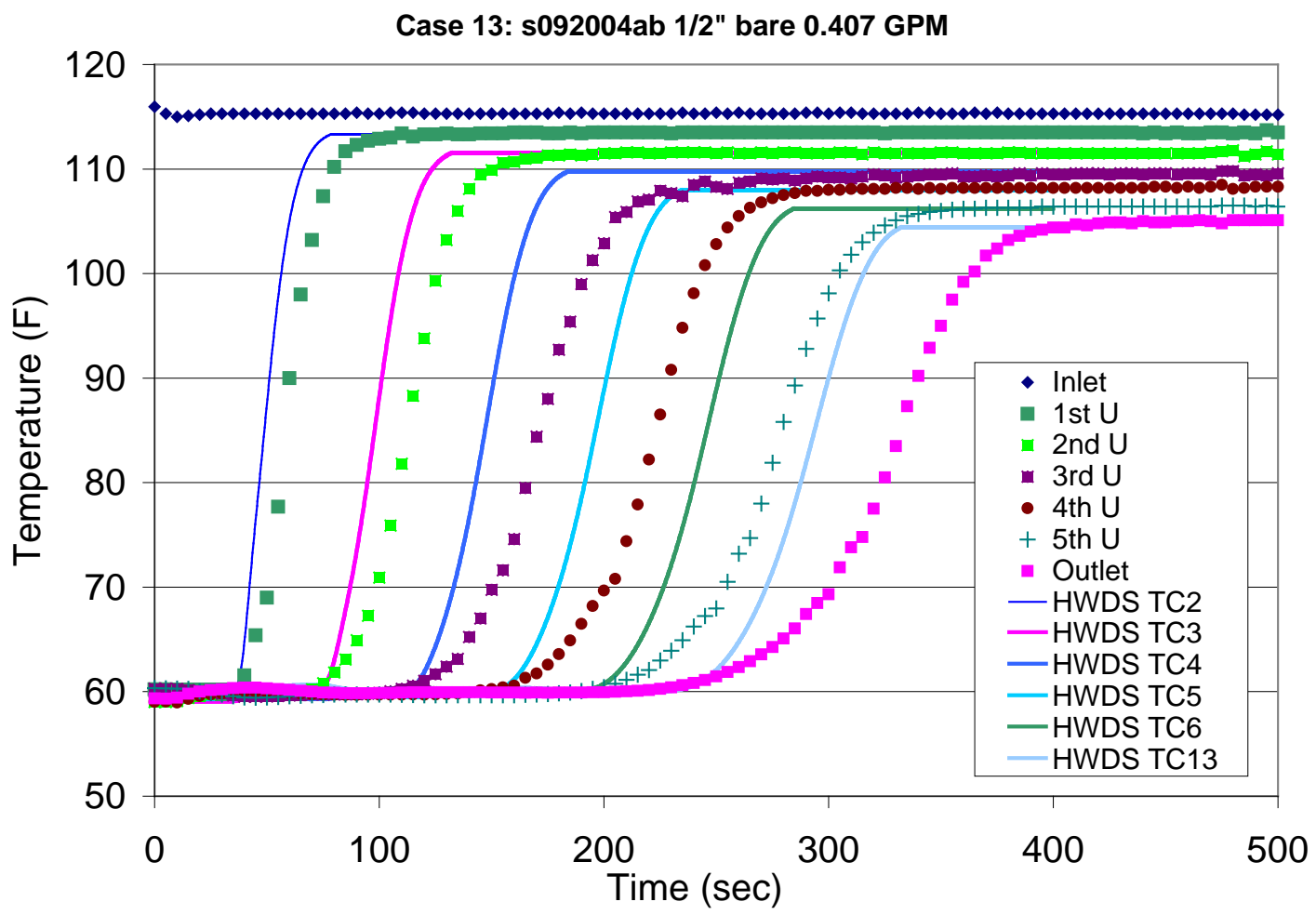

Case 14: s092004ab 1/2" bare 0.438 GPM

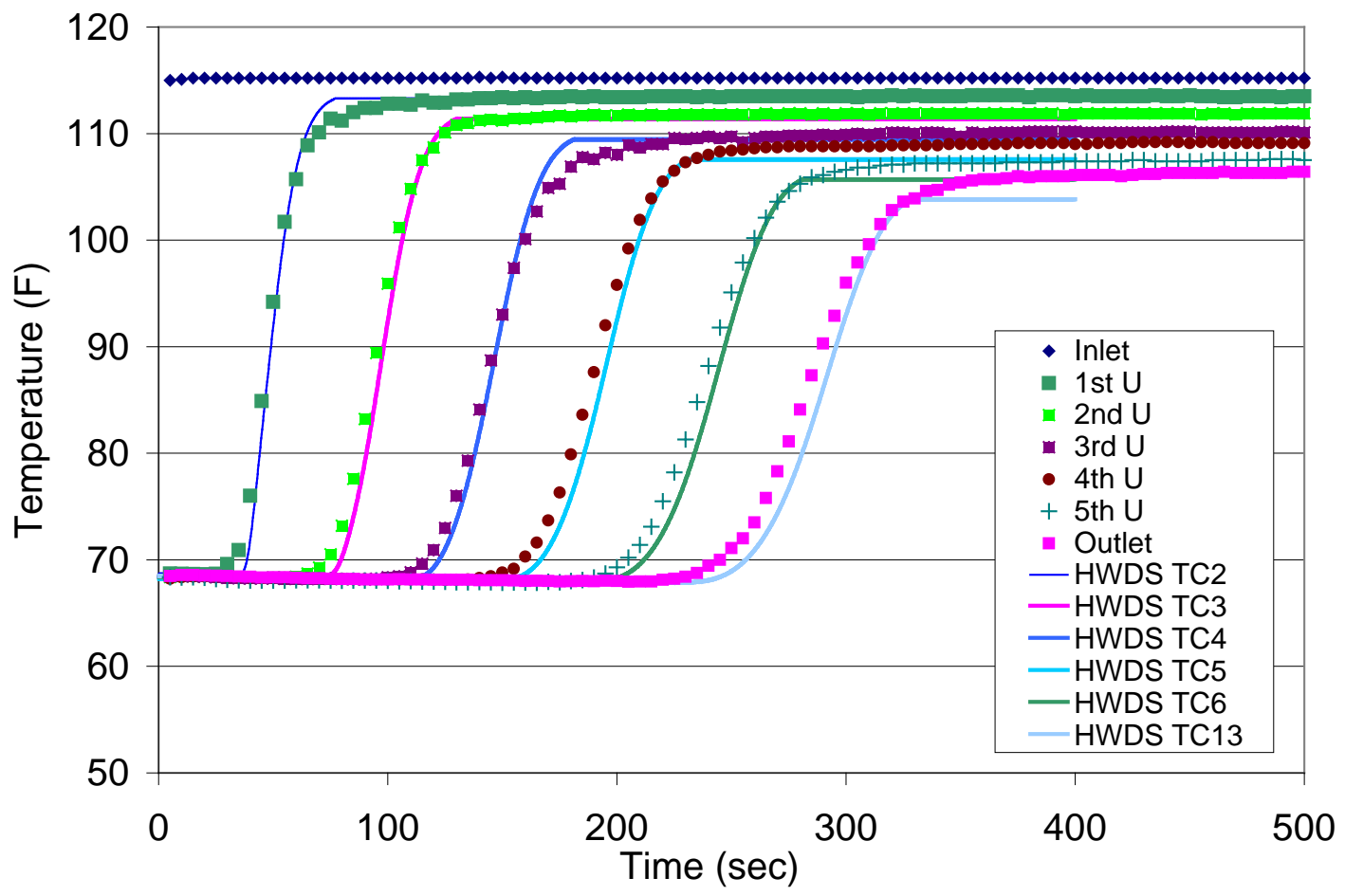



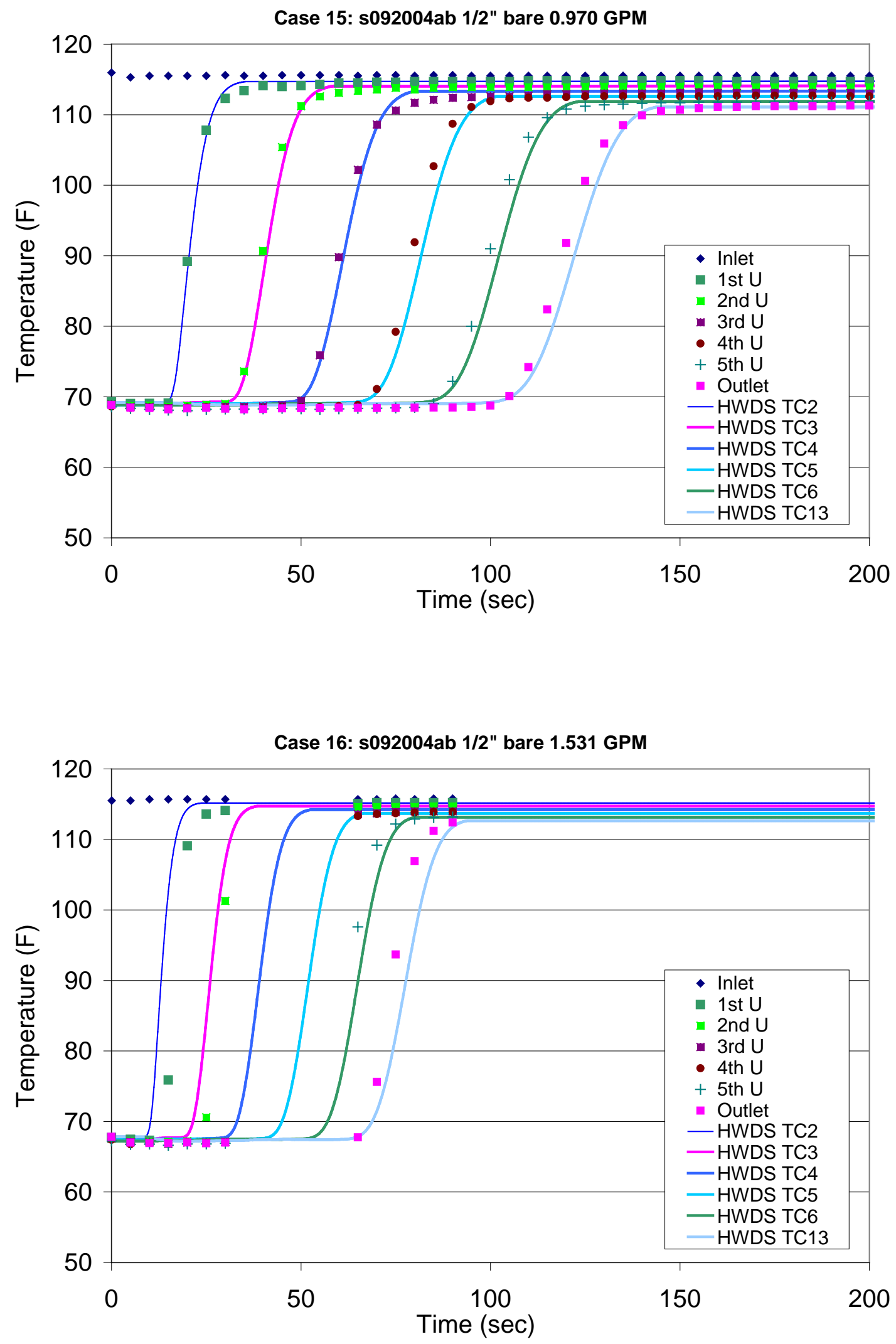

H-27 


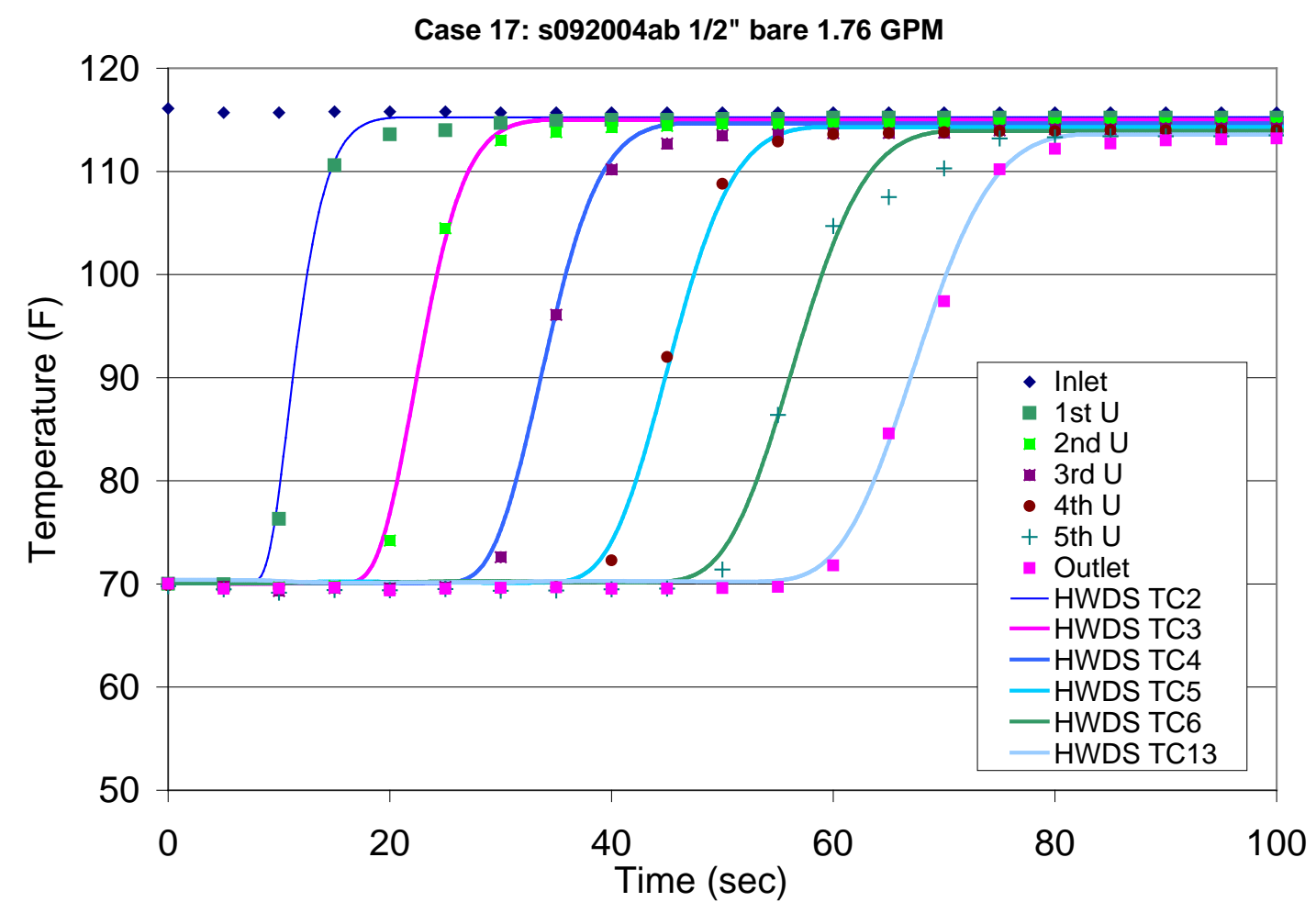

Case18: s040804a 3/4" 1.5"ins 1.28 GPM

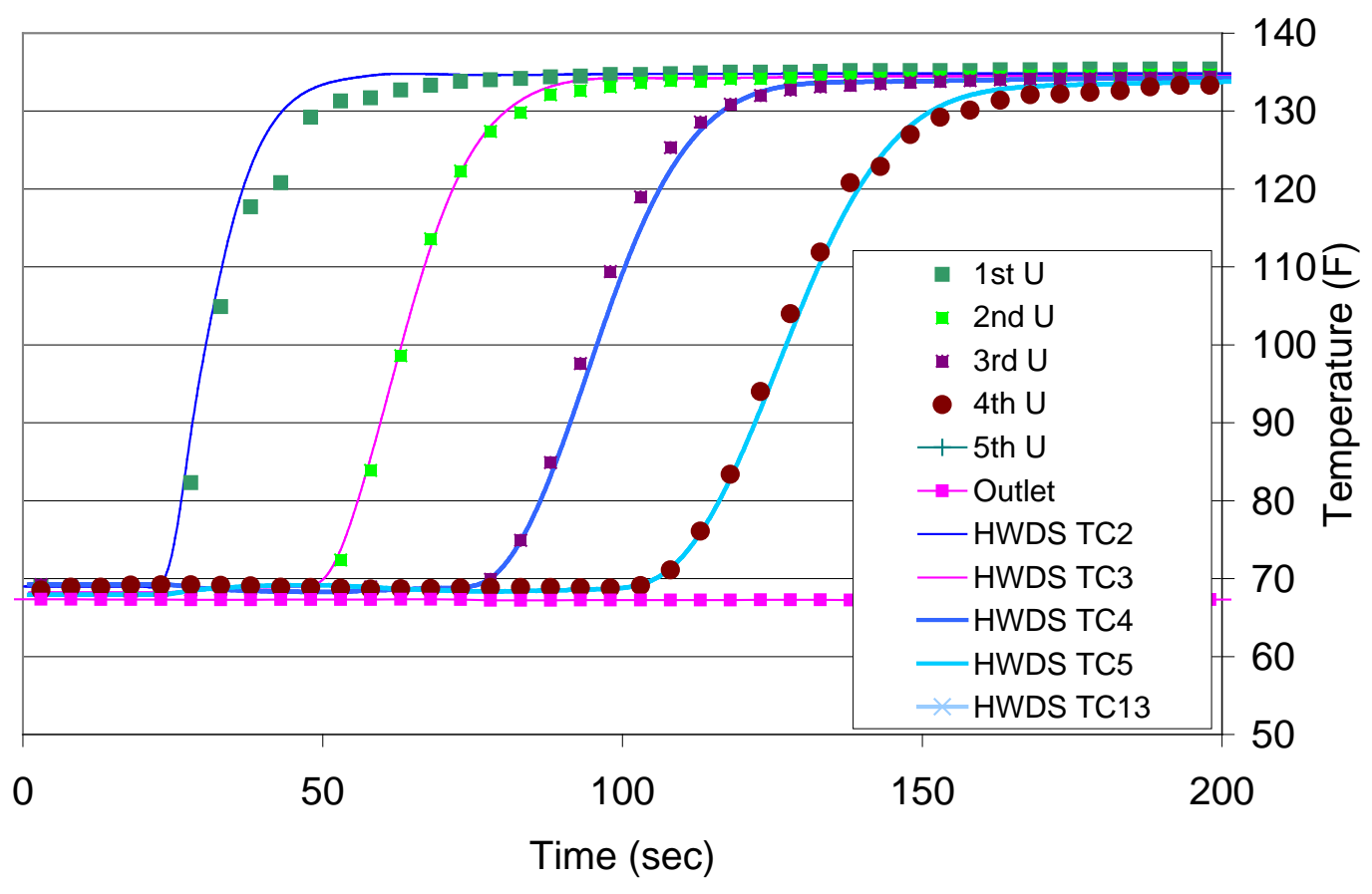


Case19: s040804a 3/4" 1.5"ins 3.16 GPM

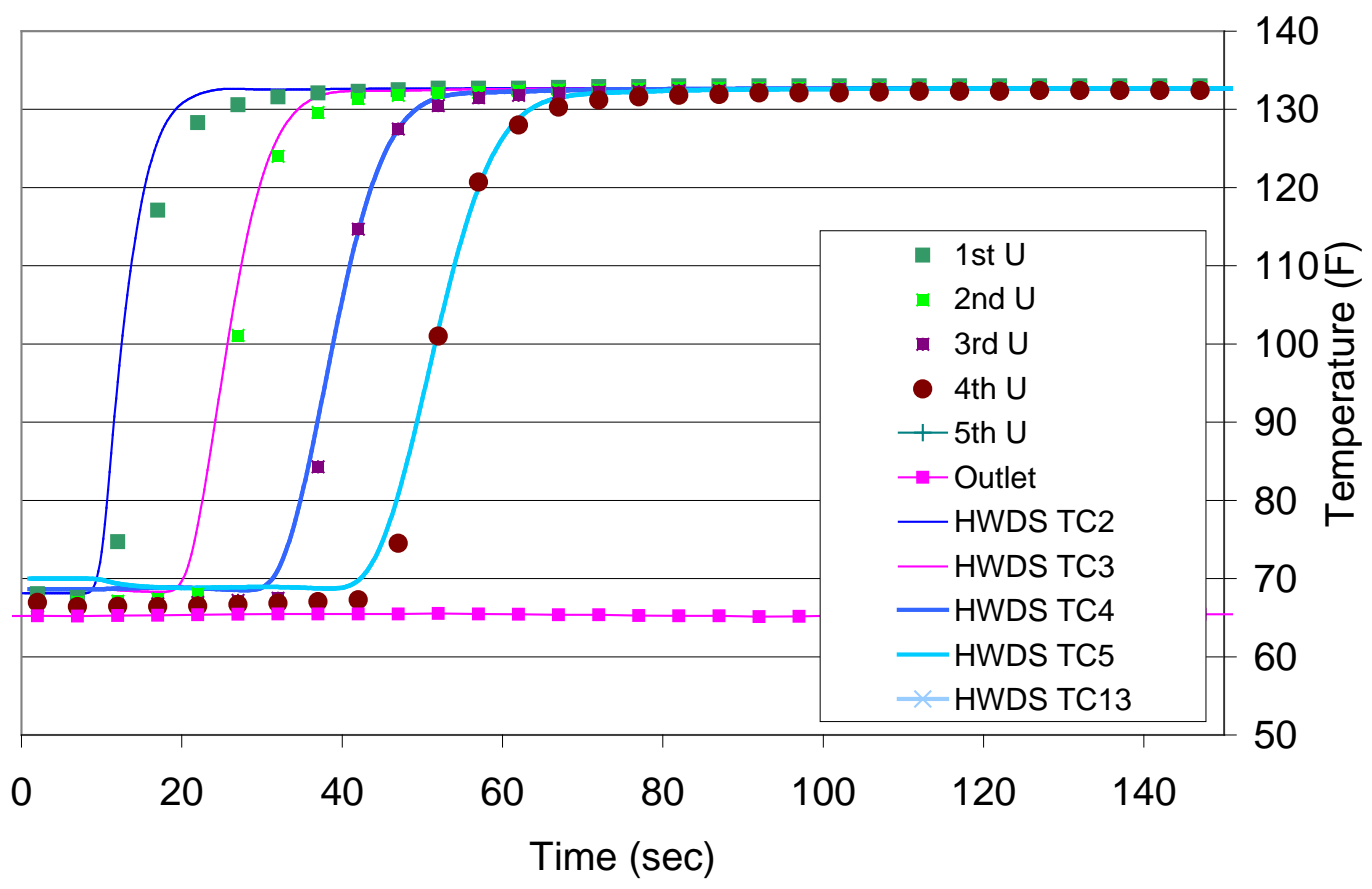

Case20: s101104a 3/4" 1.5"ins 1.77 GPM

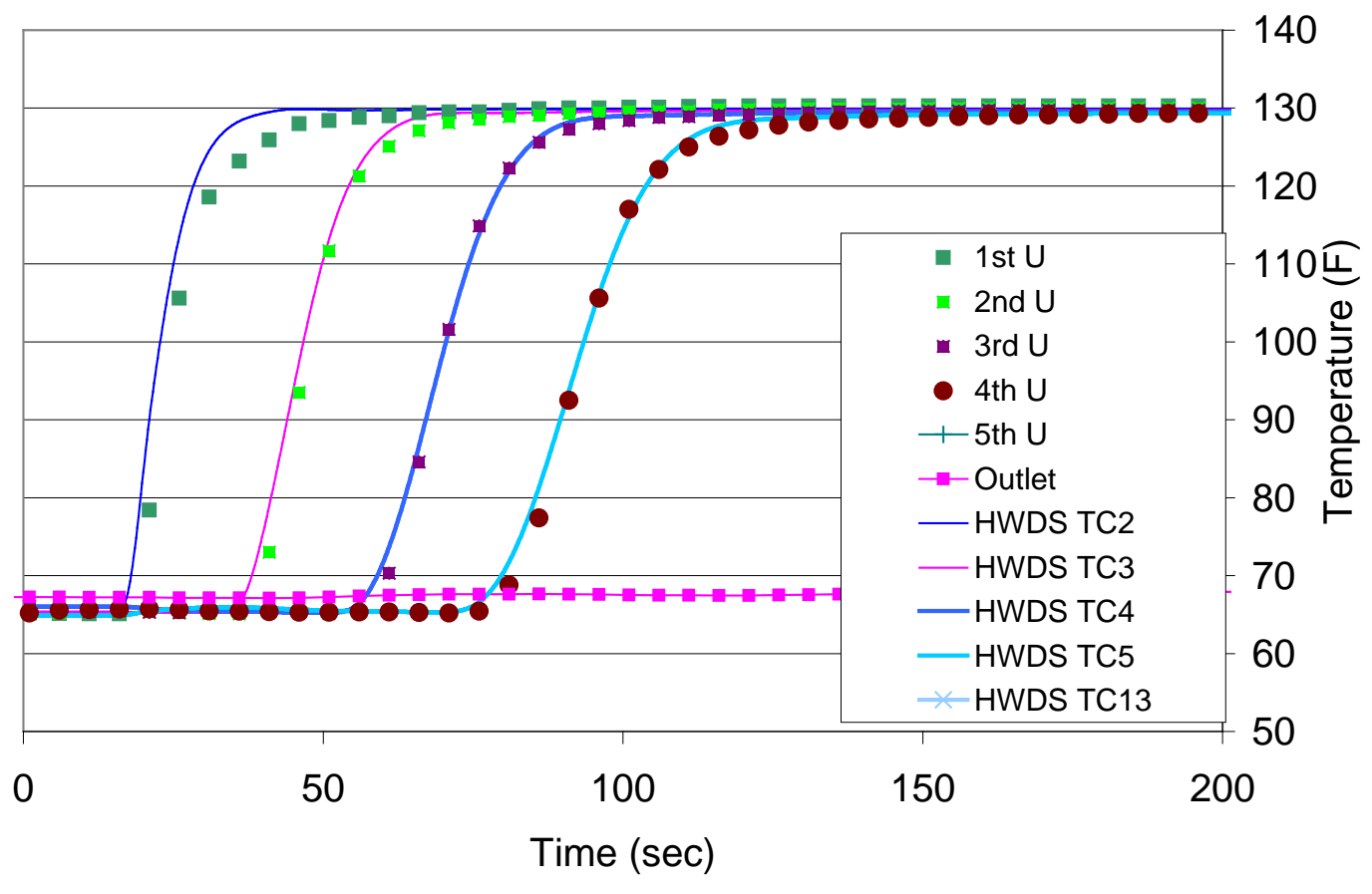


Case21: s101104a 3/4" 1.5"ins 2.764 GPM

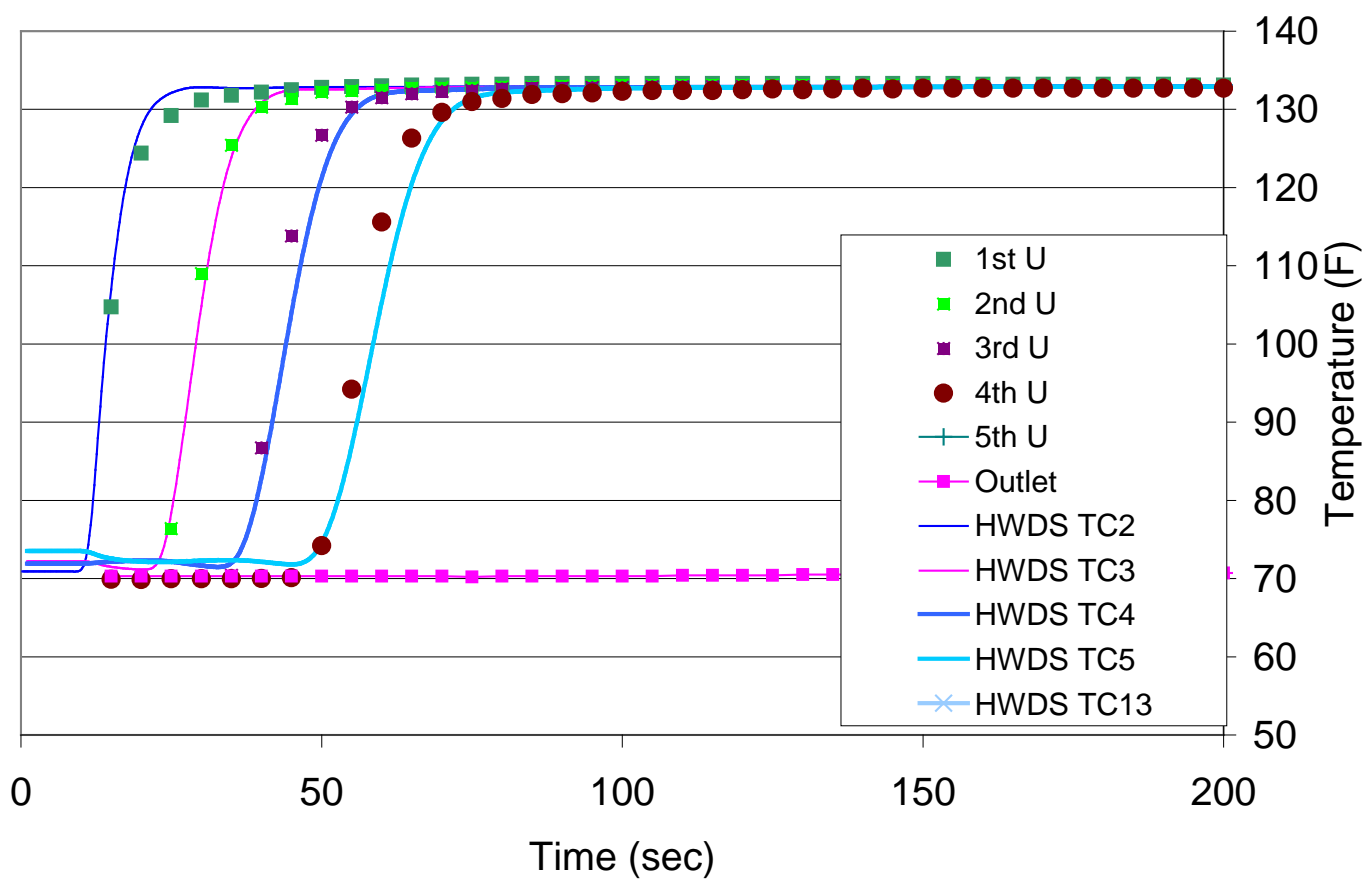

Case22: s101104a 3/4" 1.5"ins 4.37 GPM

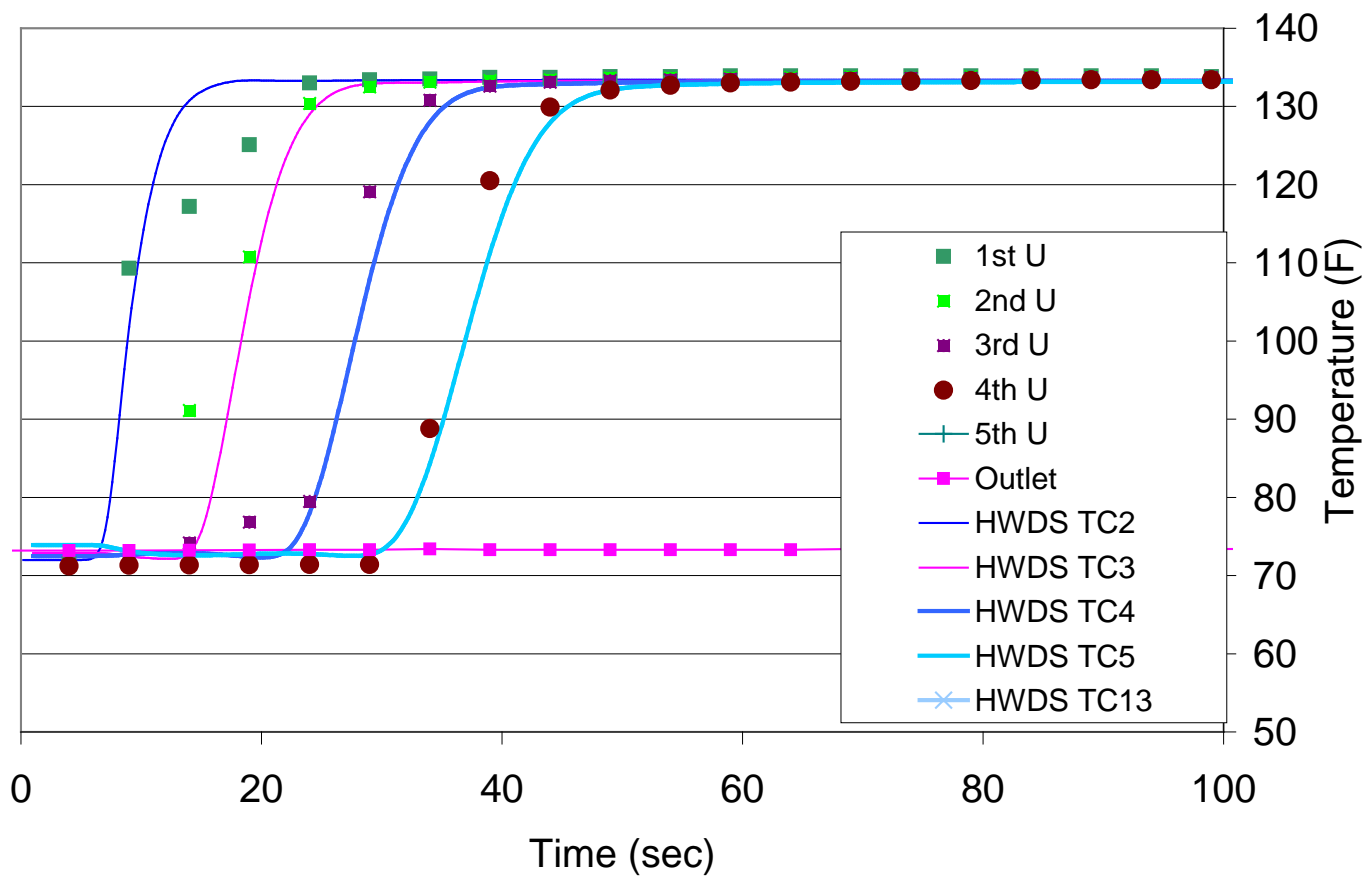


Case23: s101104a 3/4" 1.5"ins 3.625 GPM
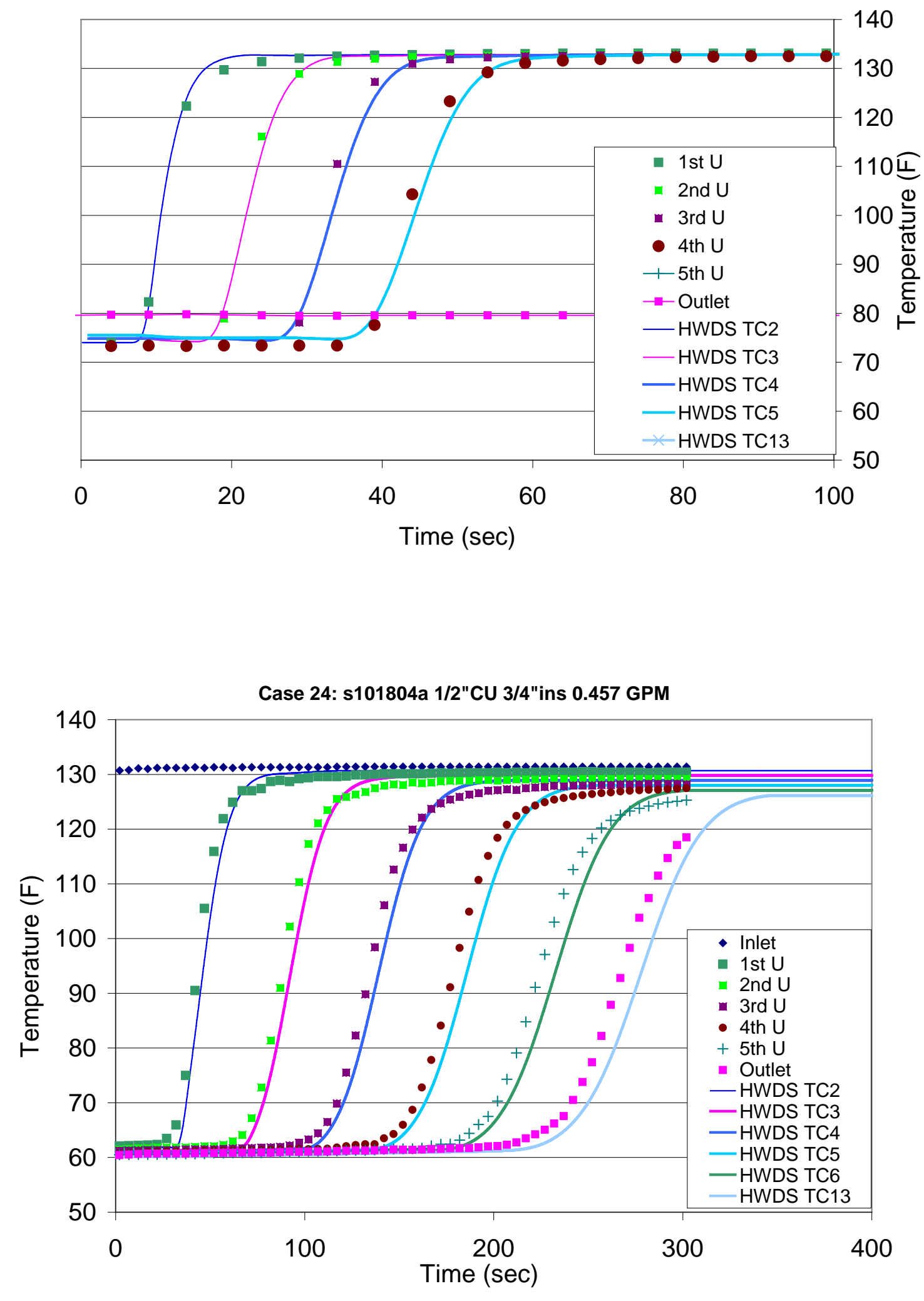

H-31 

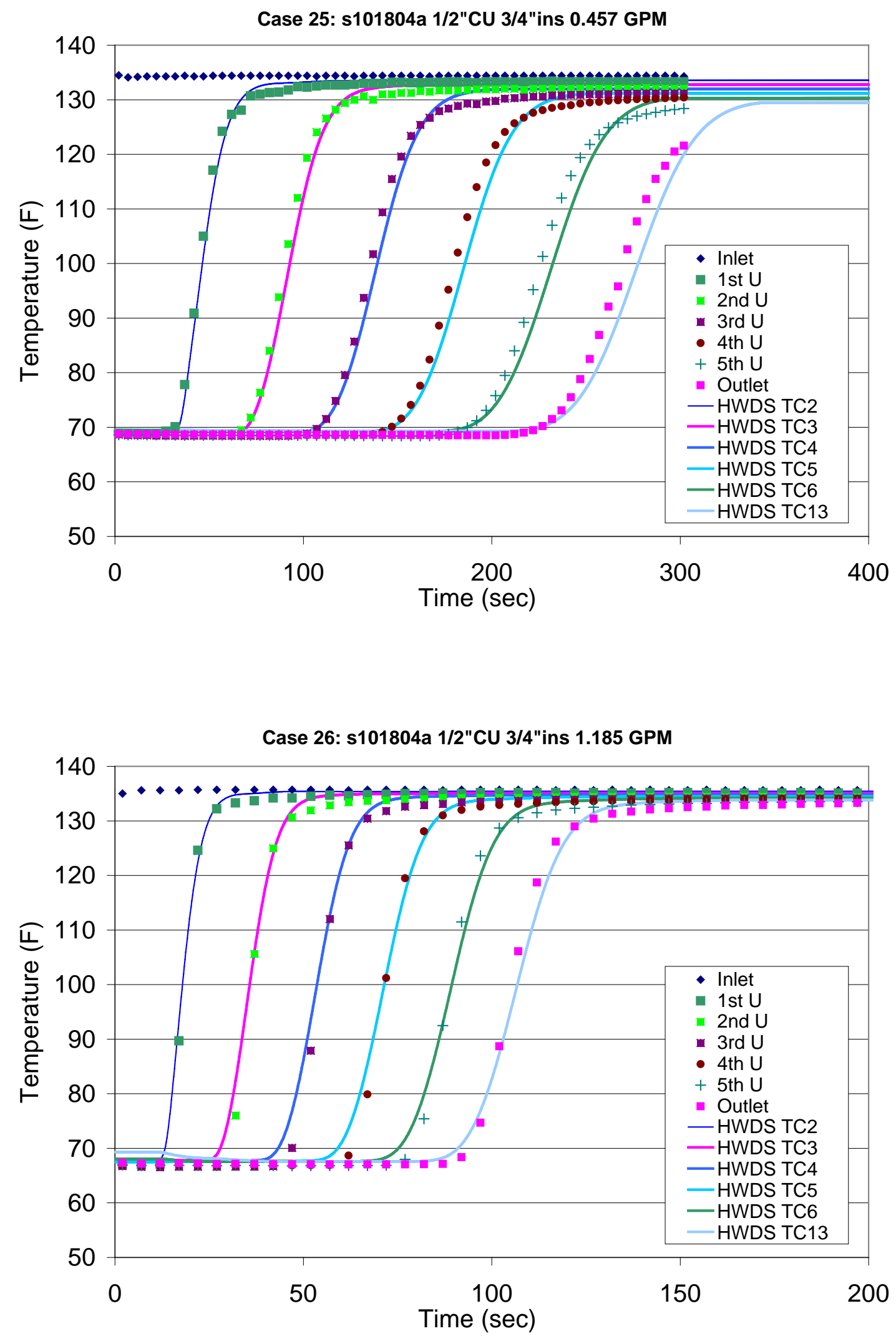

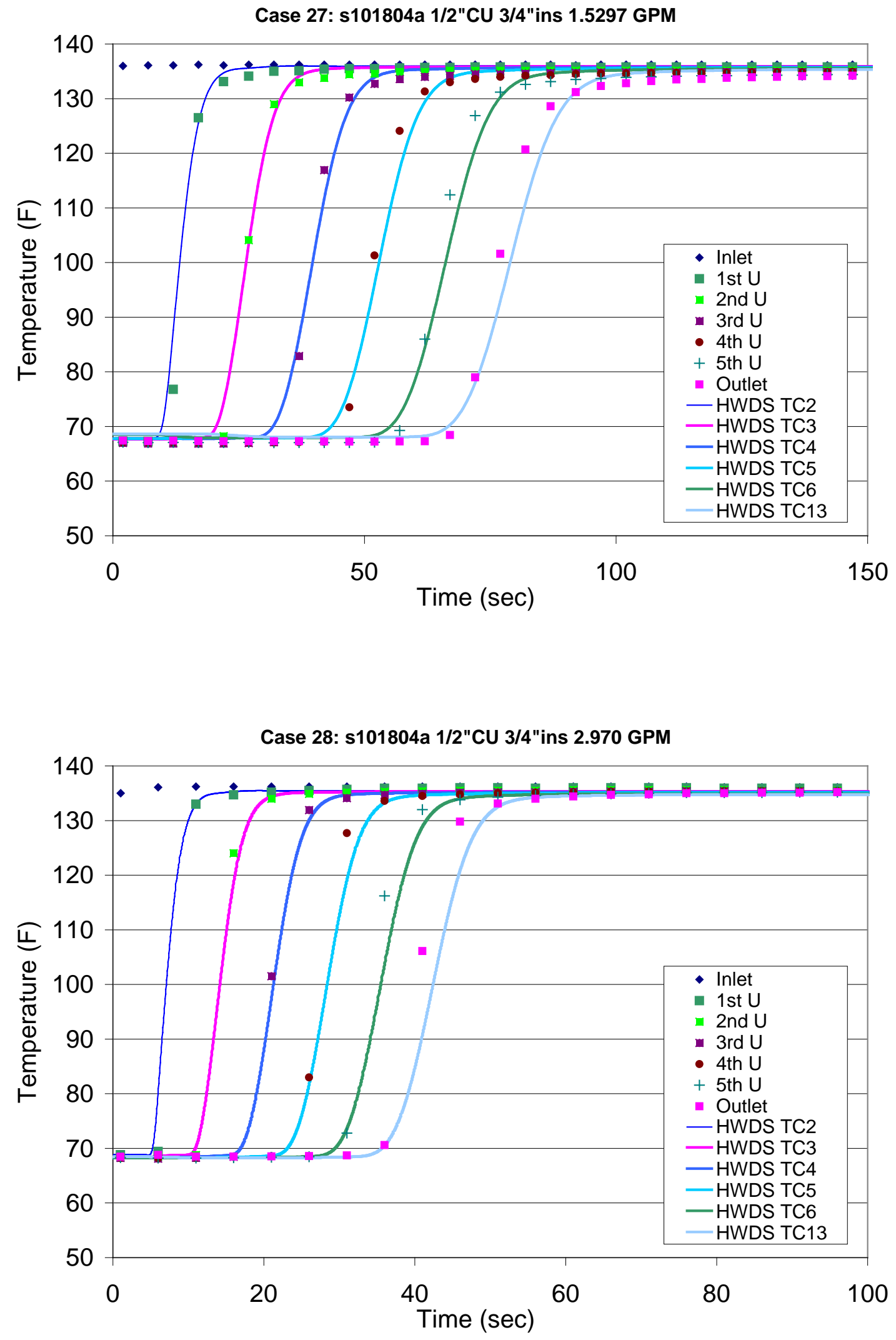

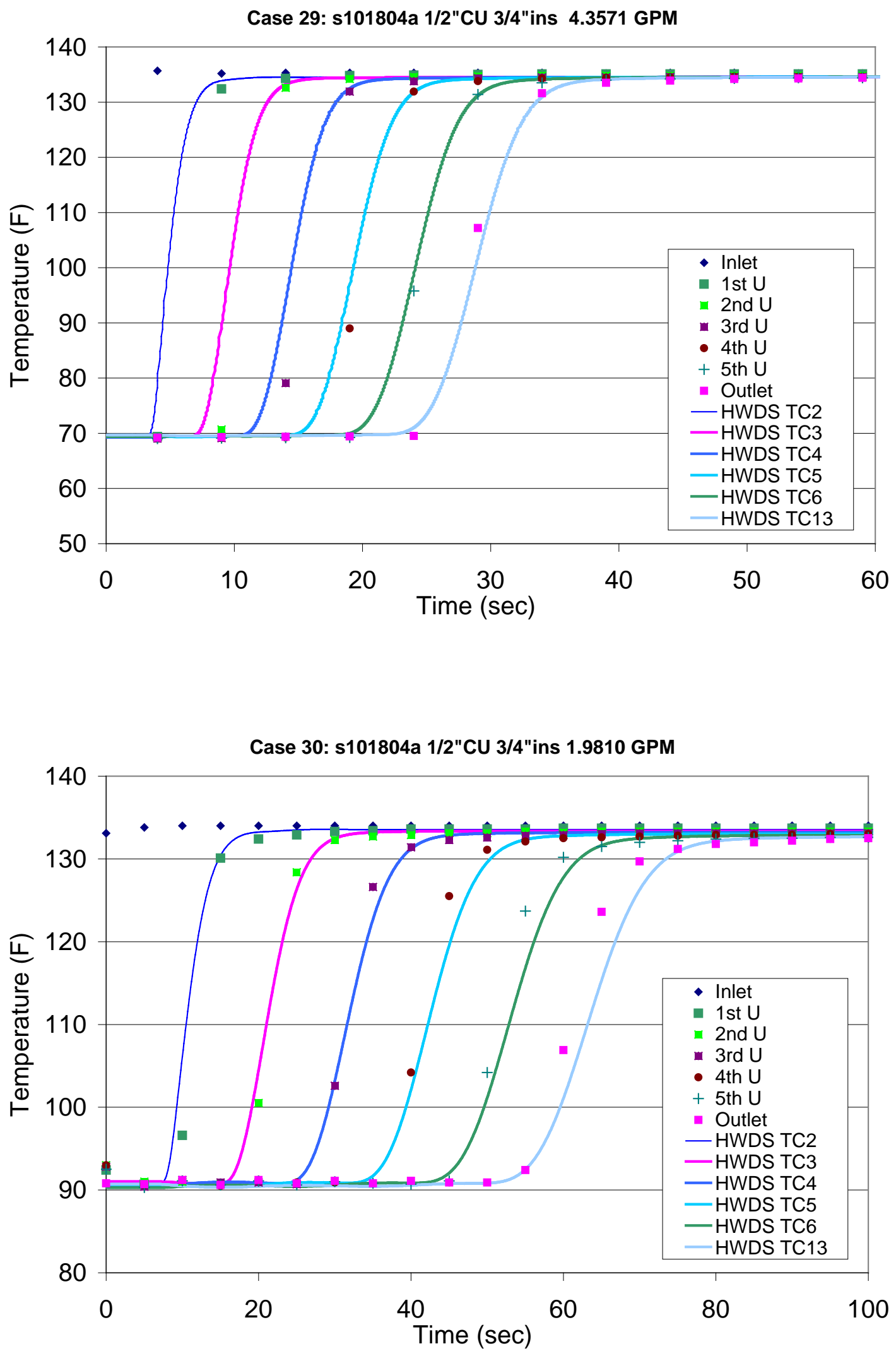

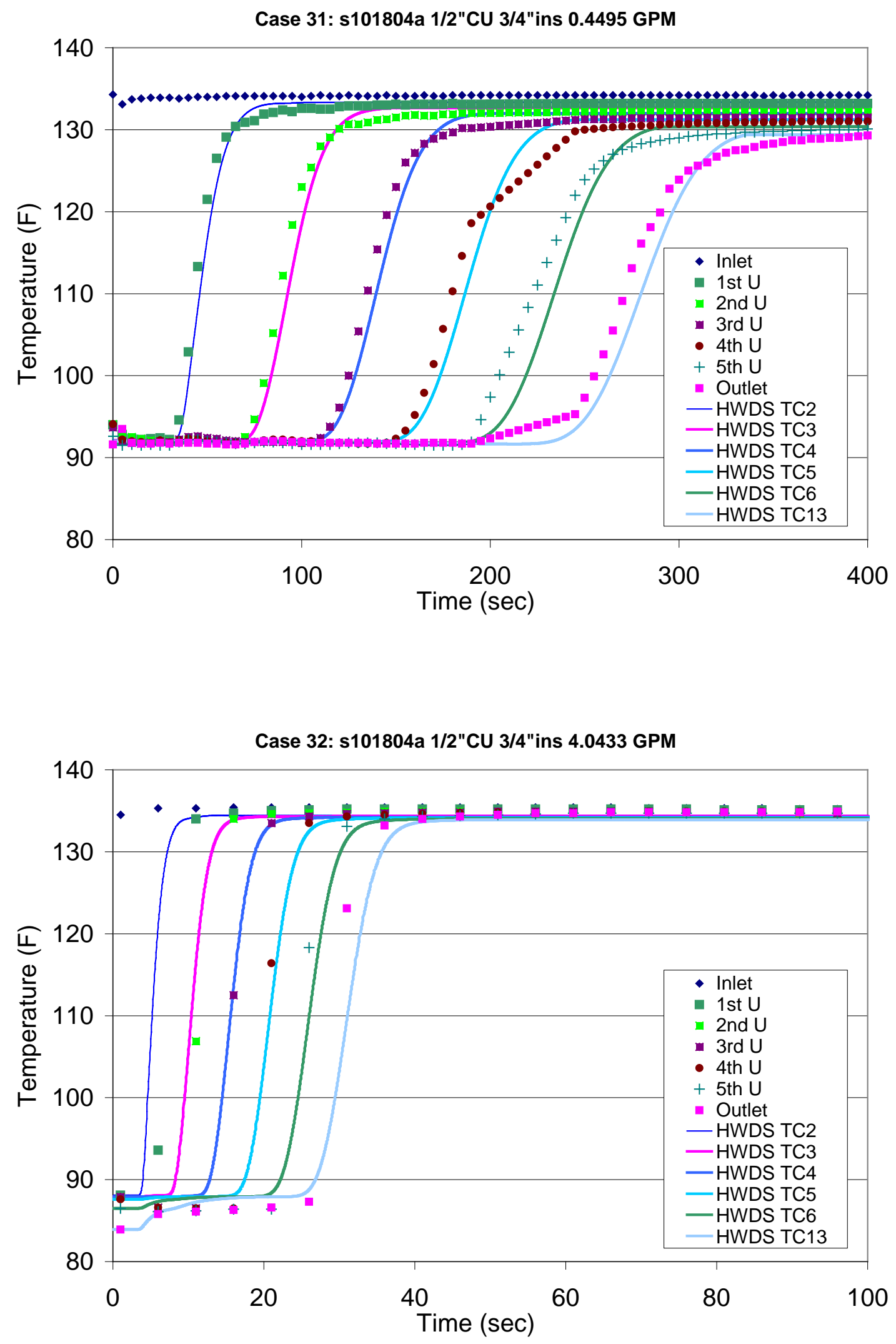

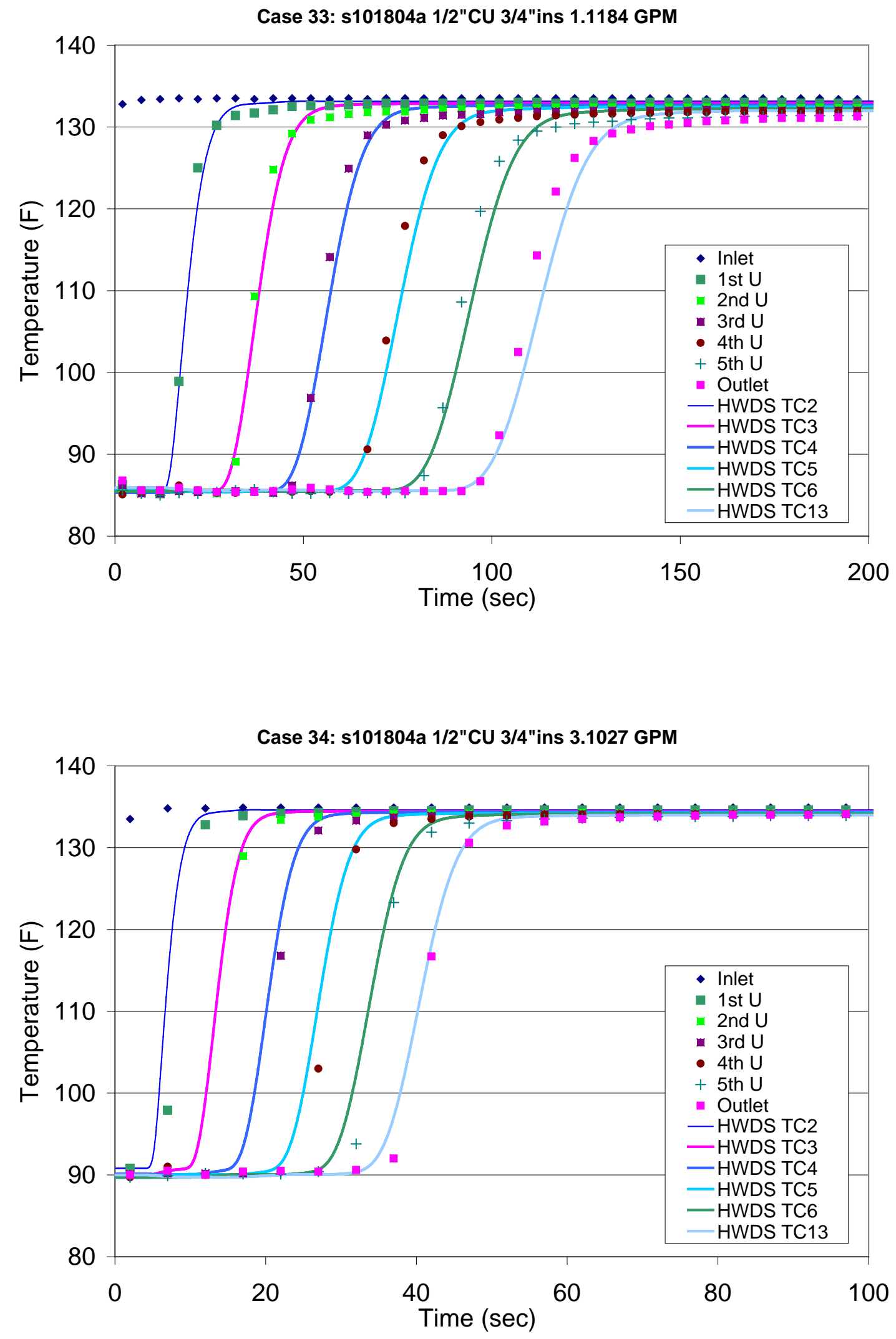

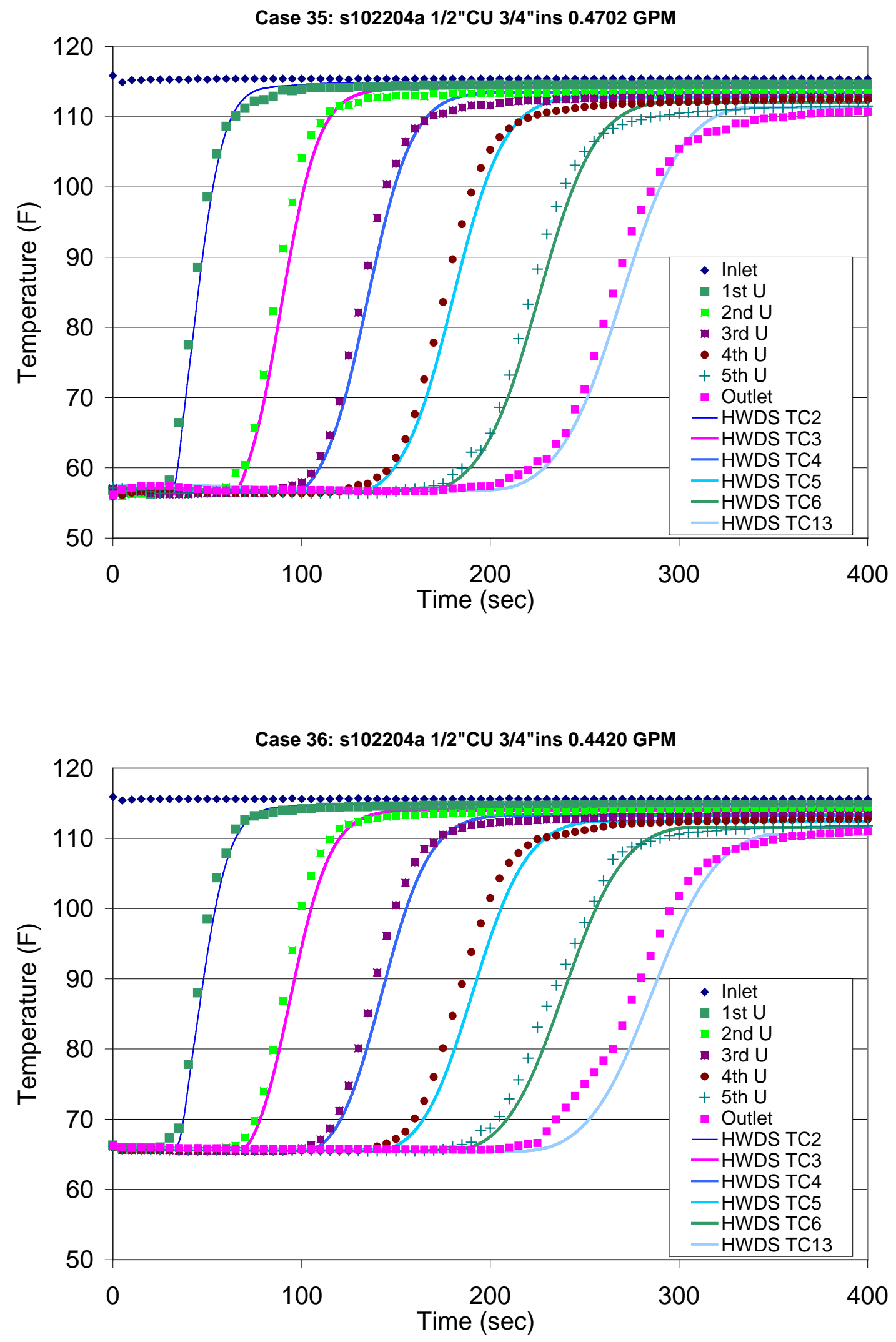


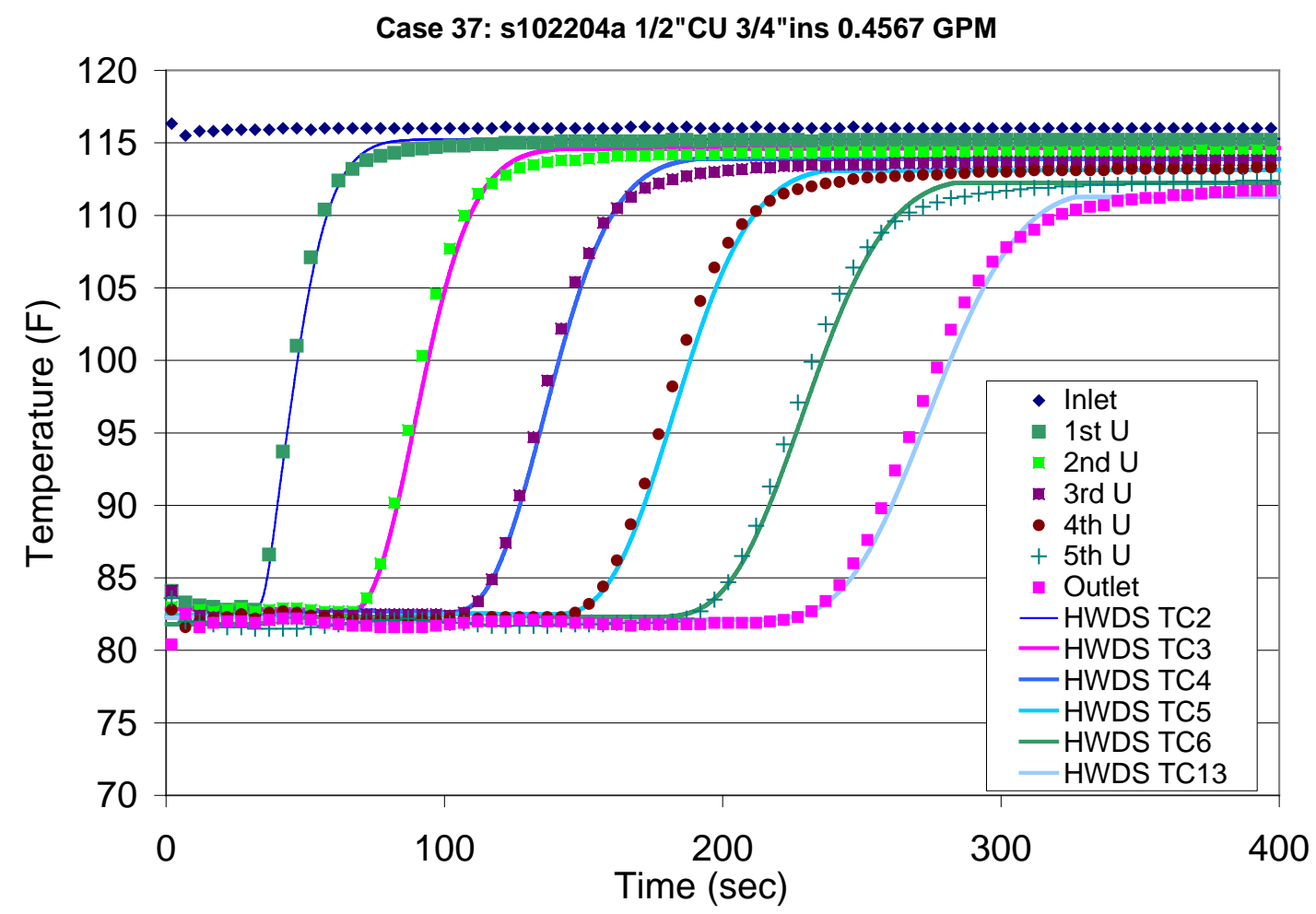



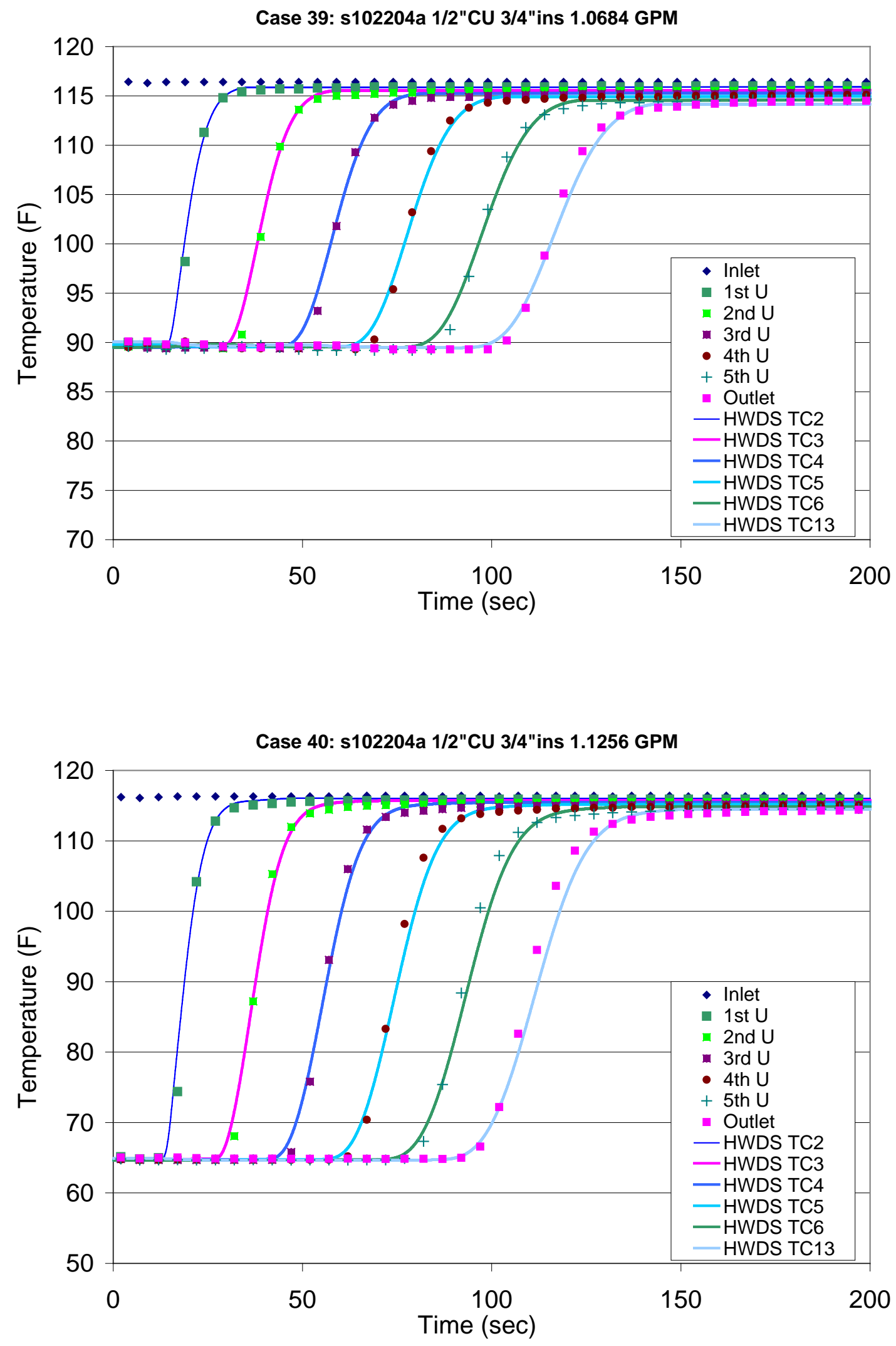

H-39 

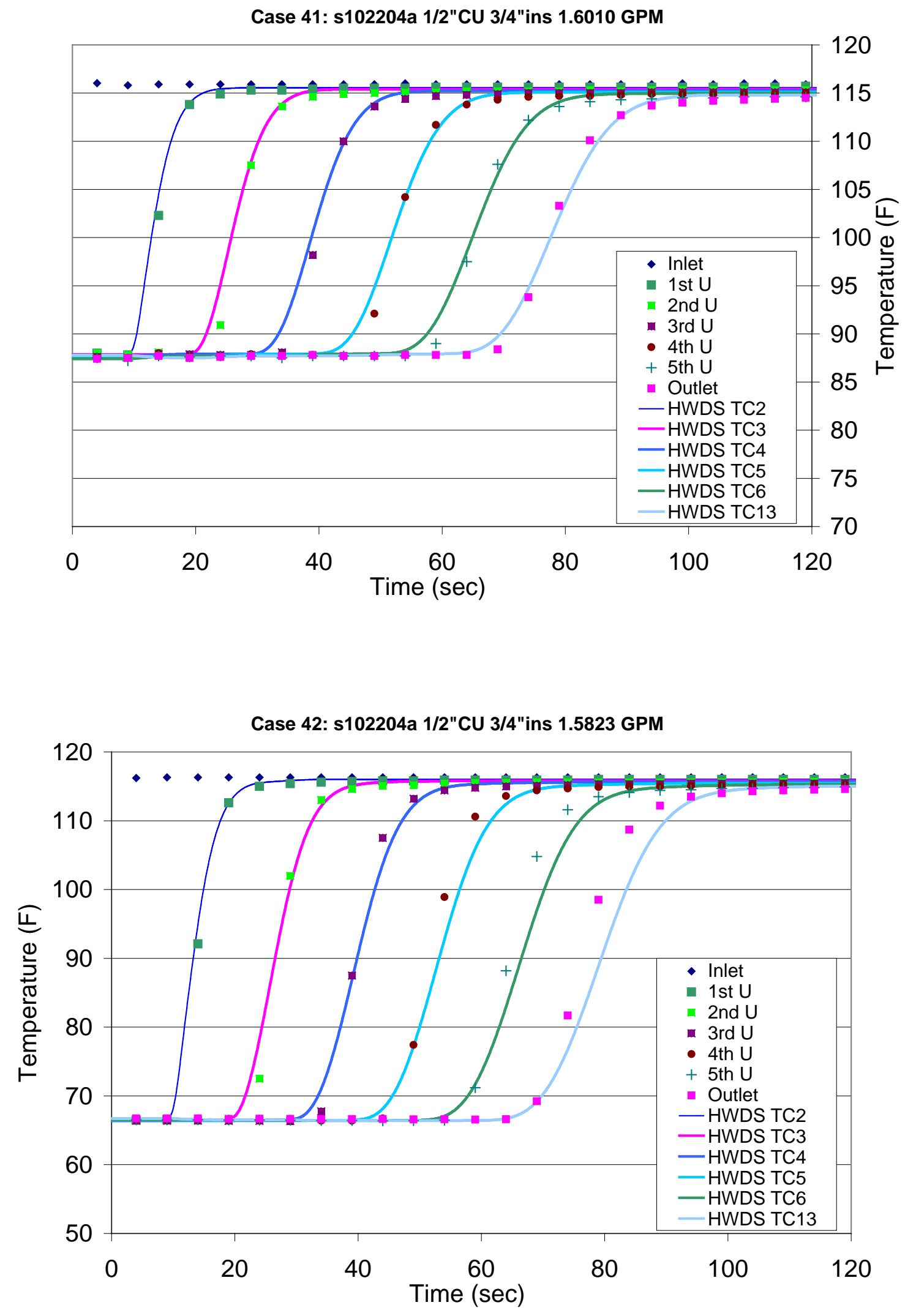

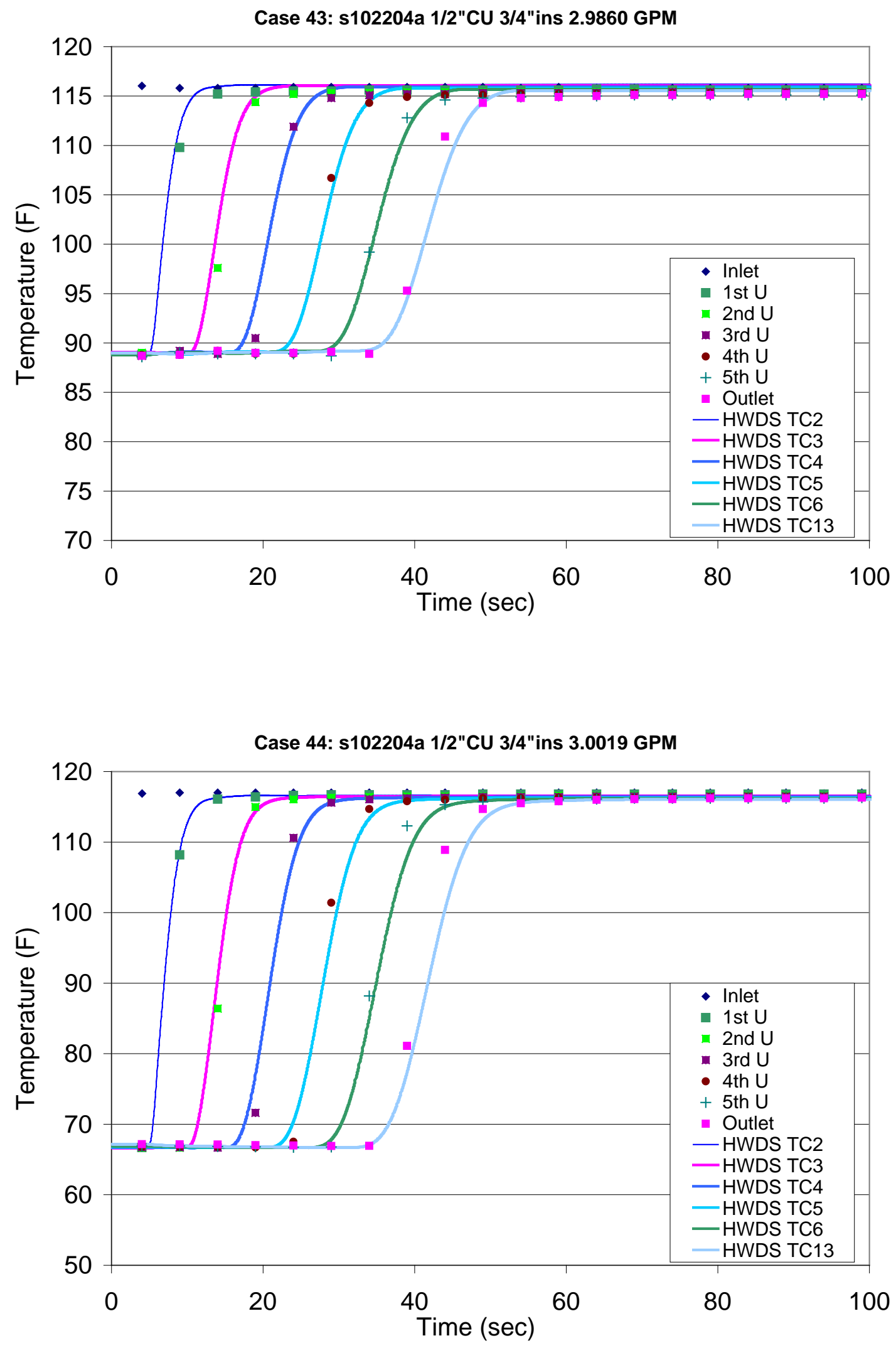

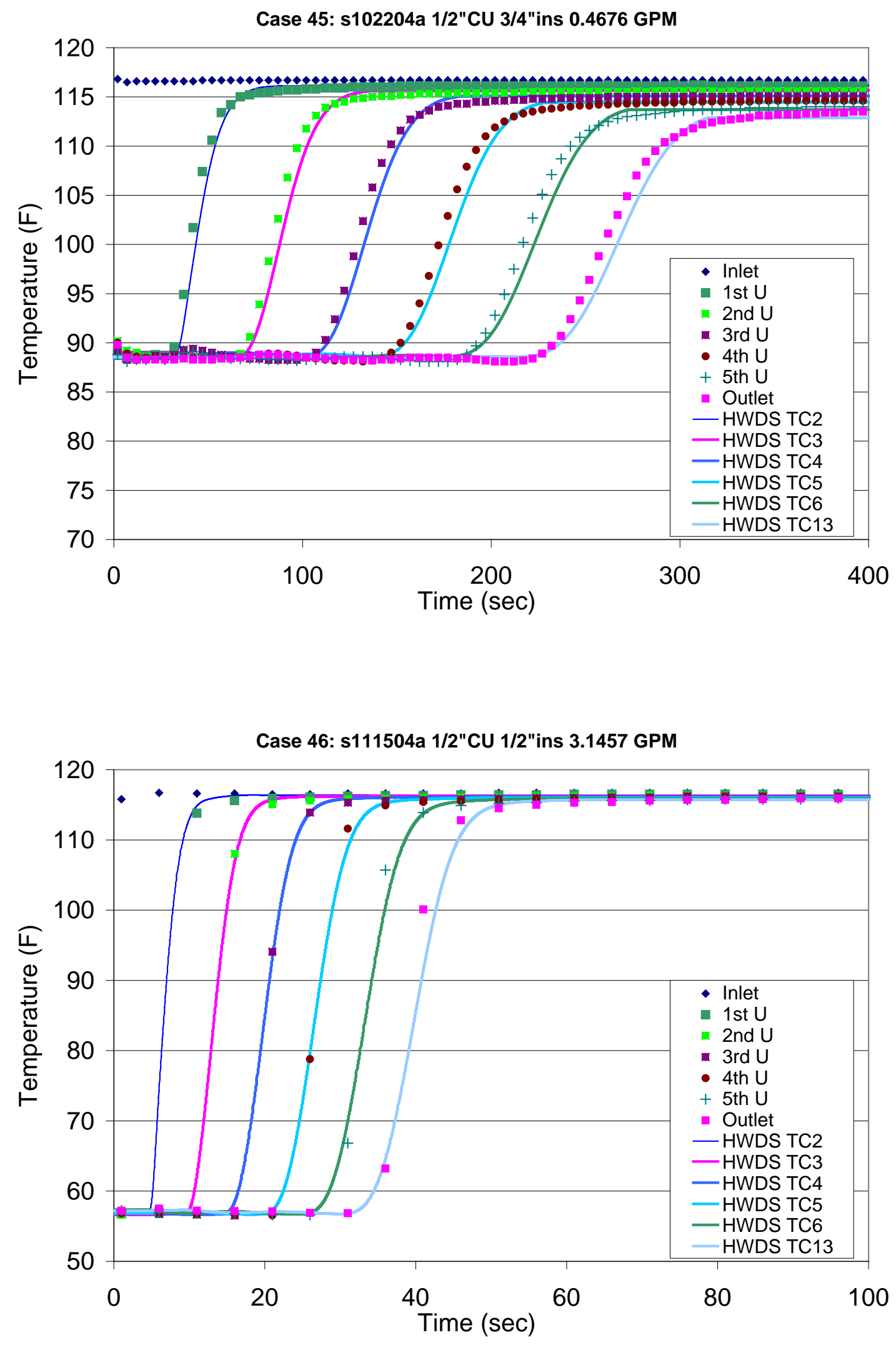

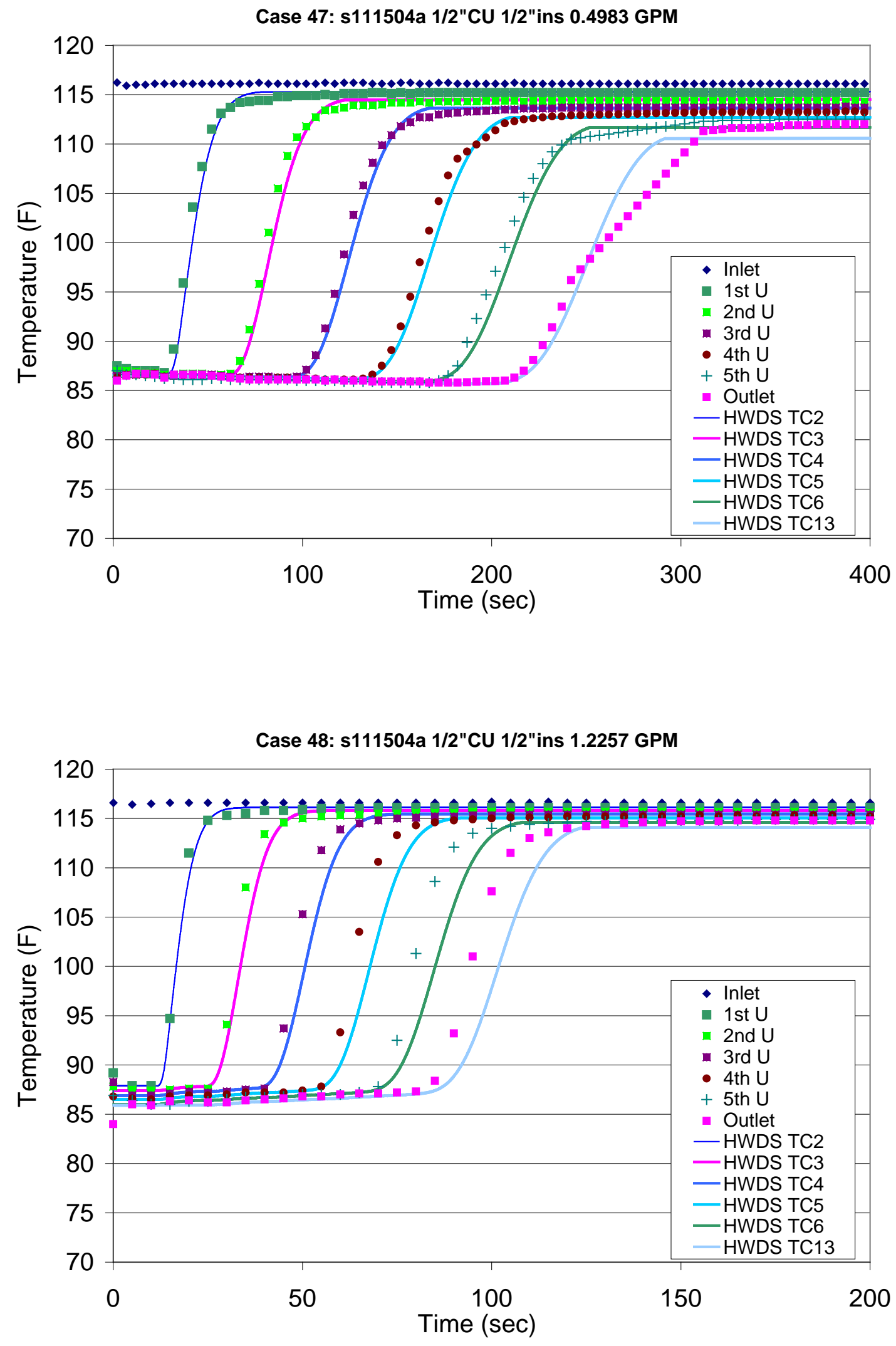

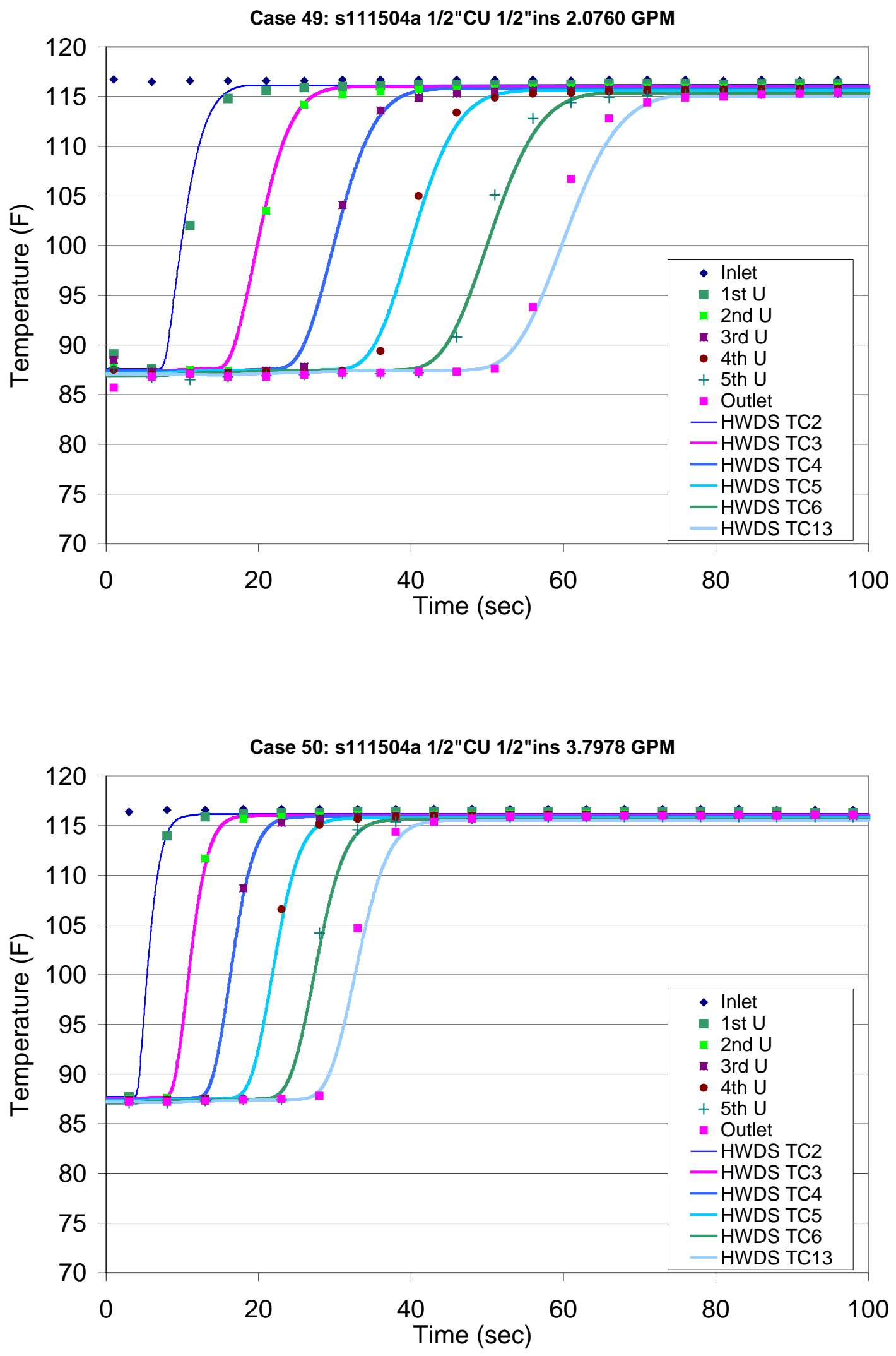

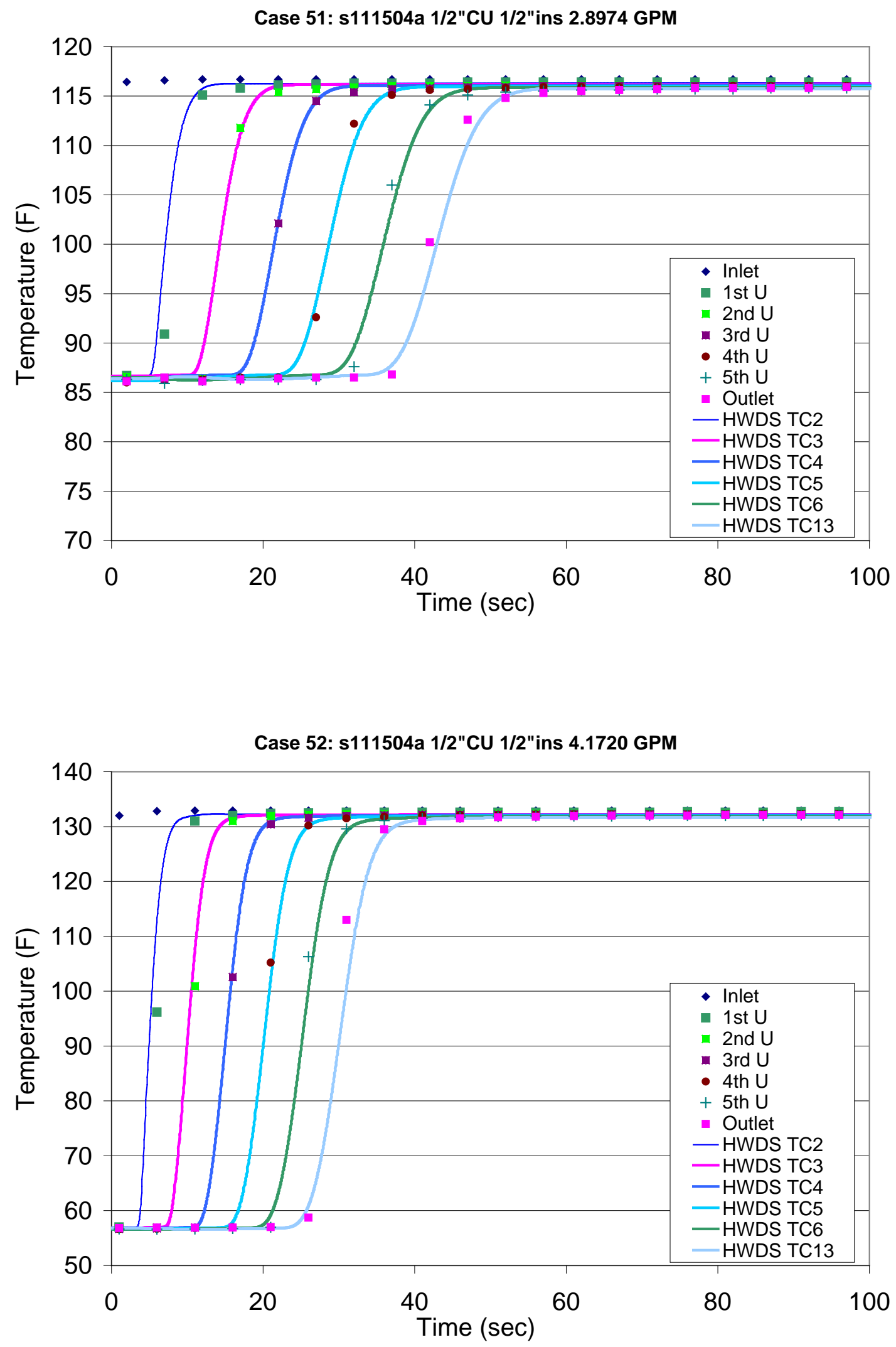

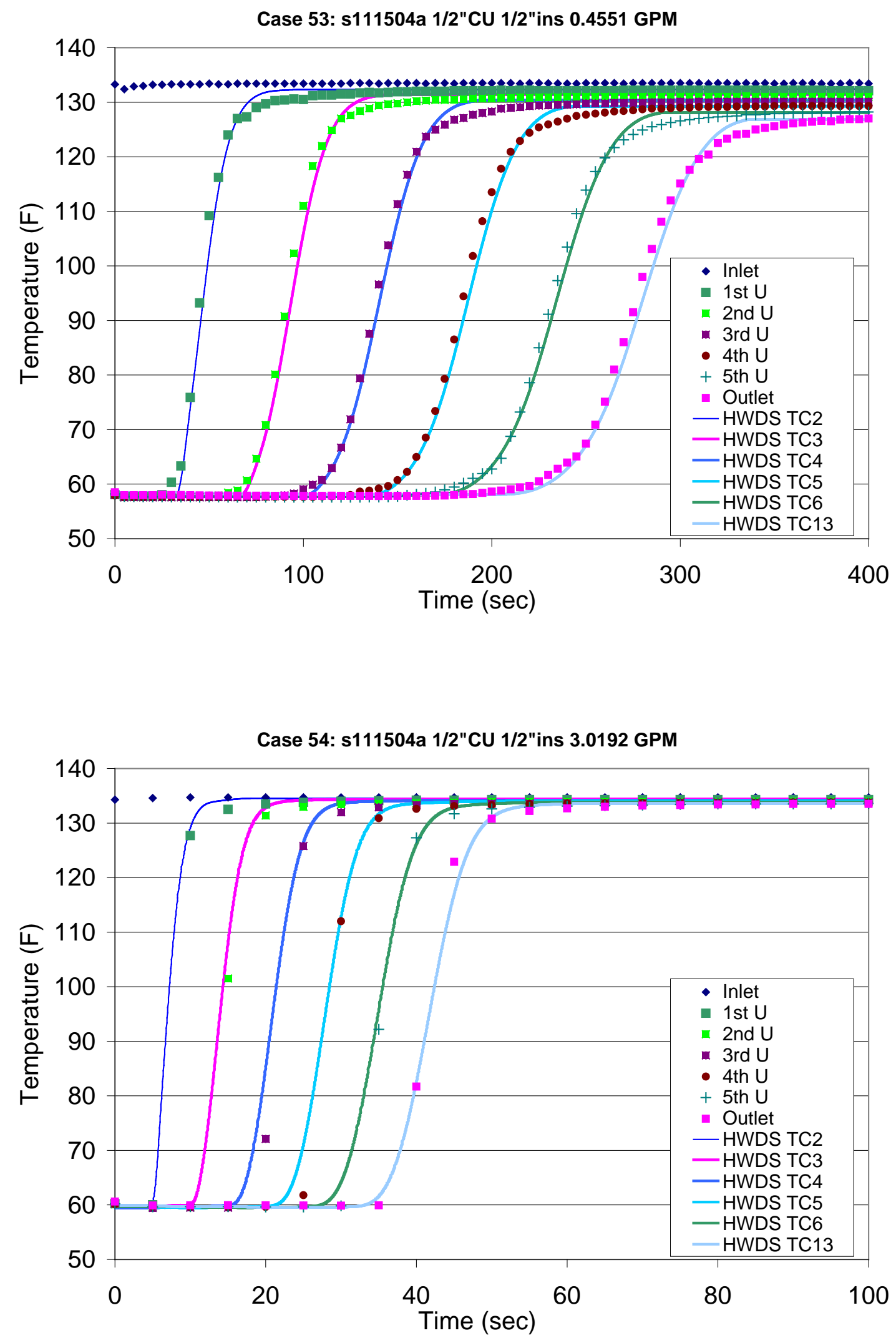

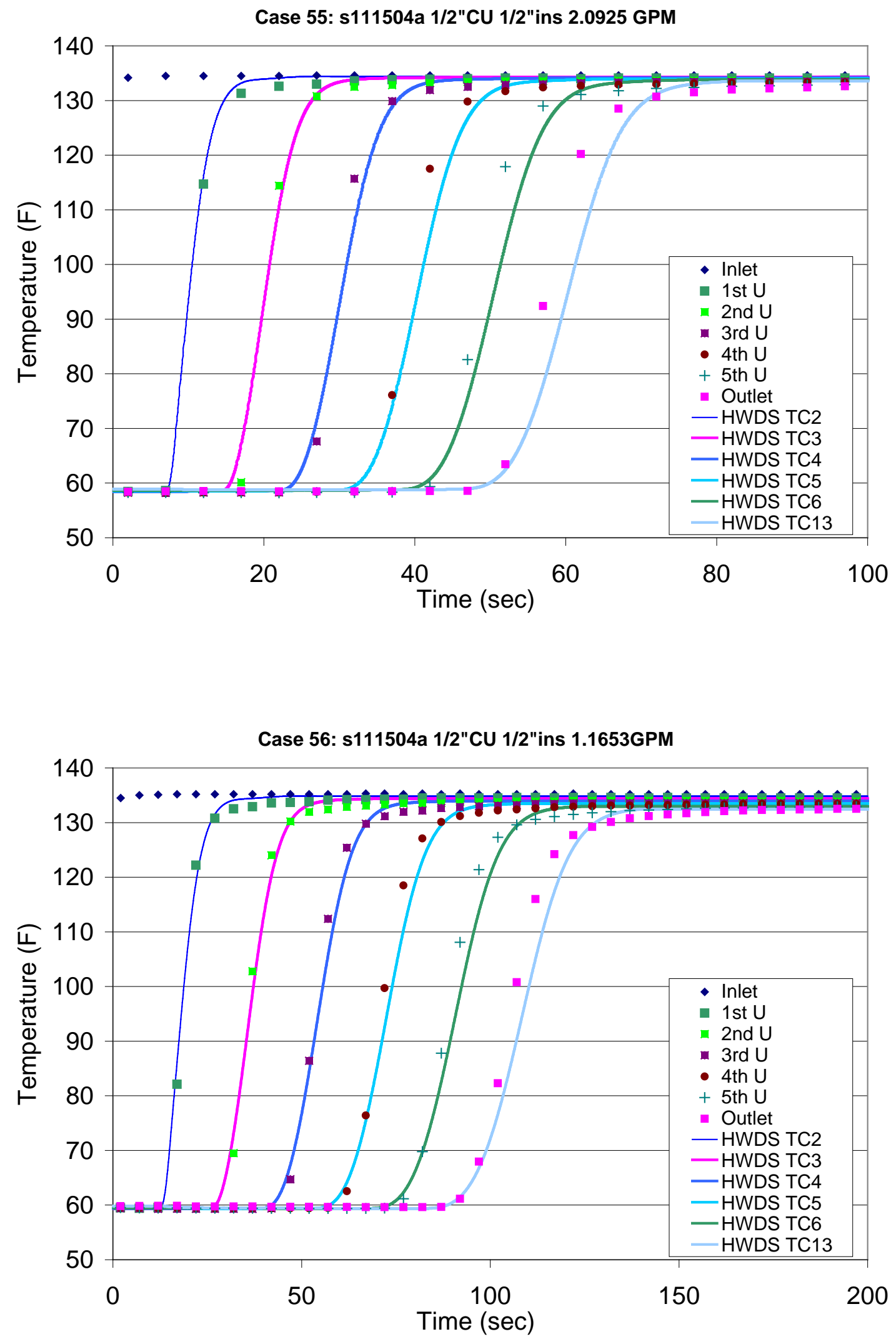

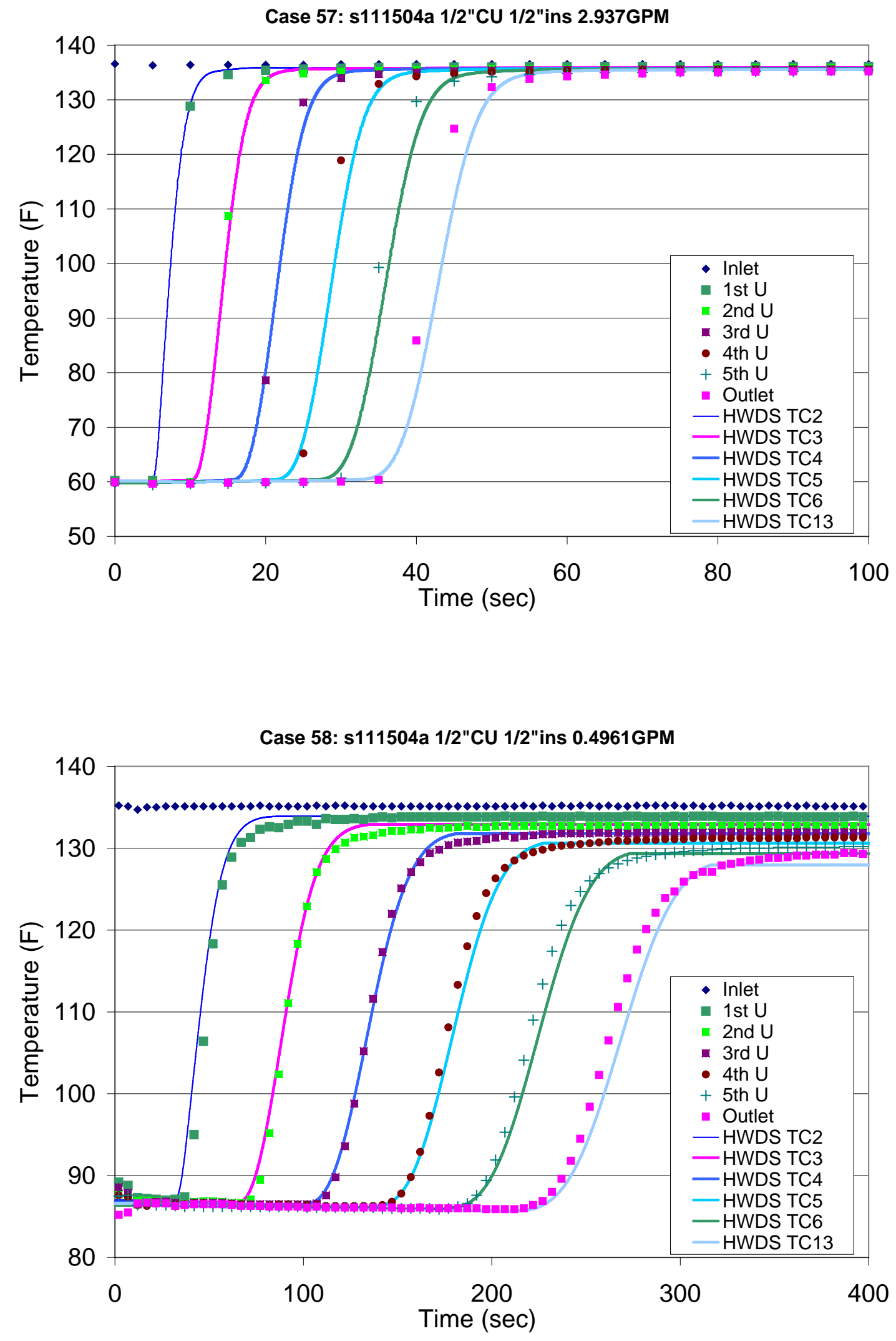

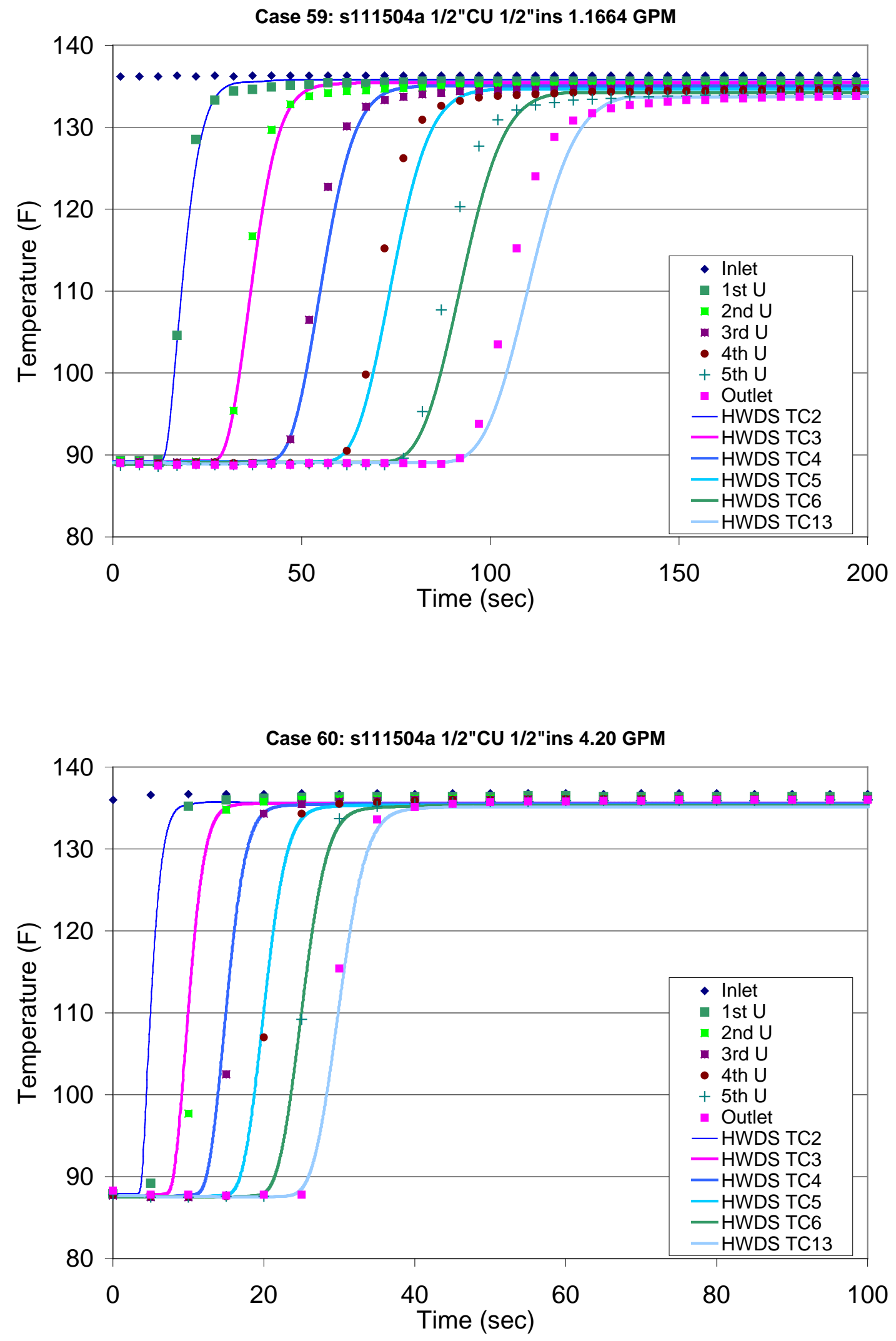


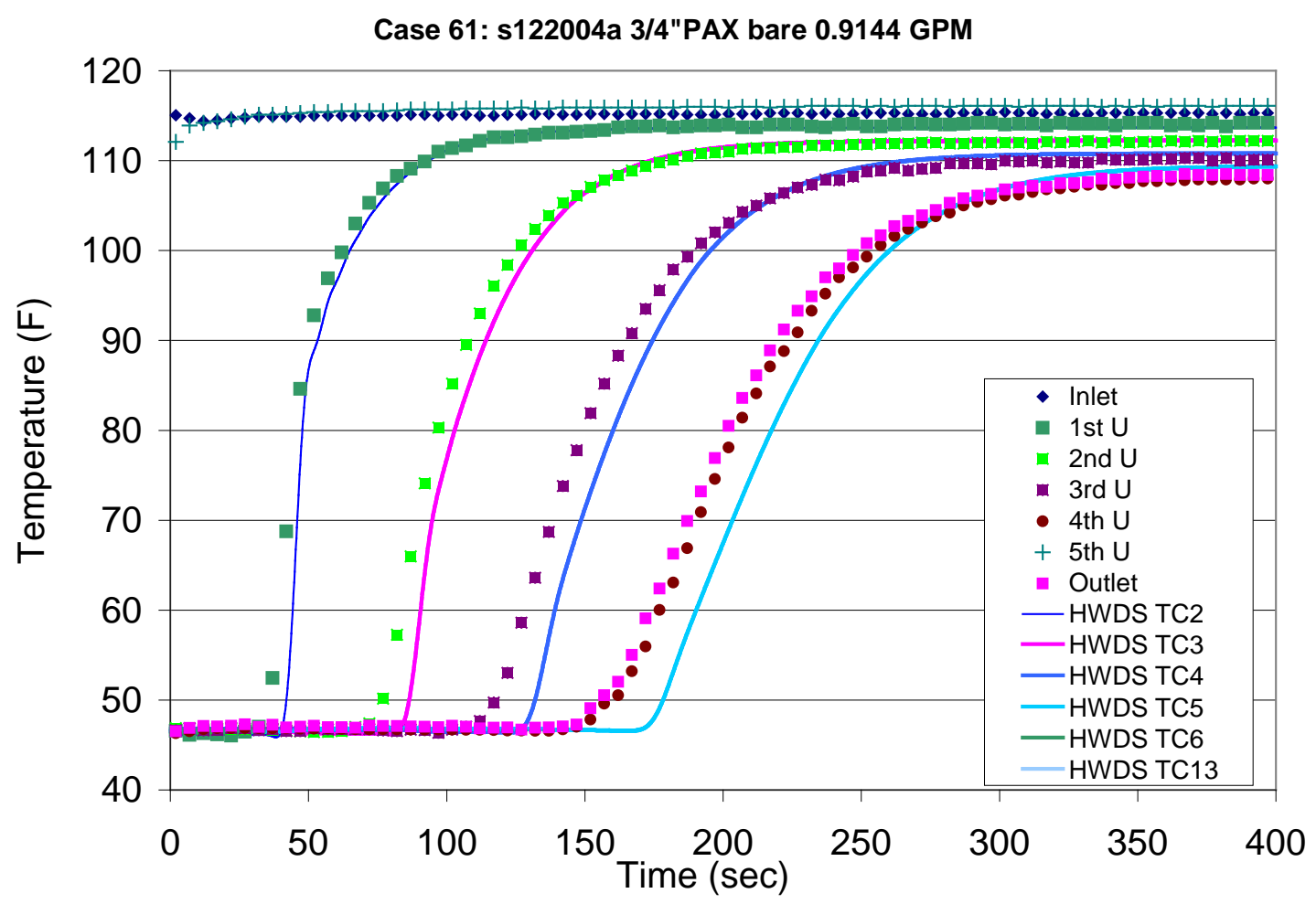

Case 62: s122004a 3/4"PAX bare 4.4804 GPM

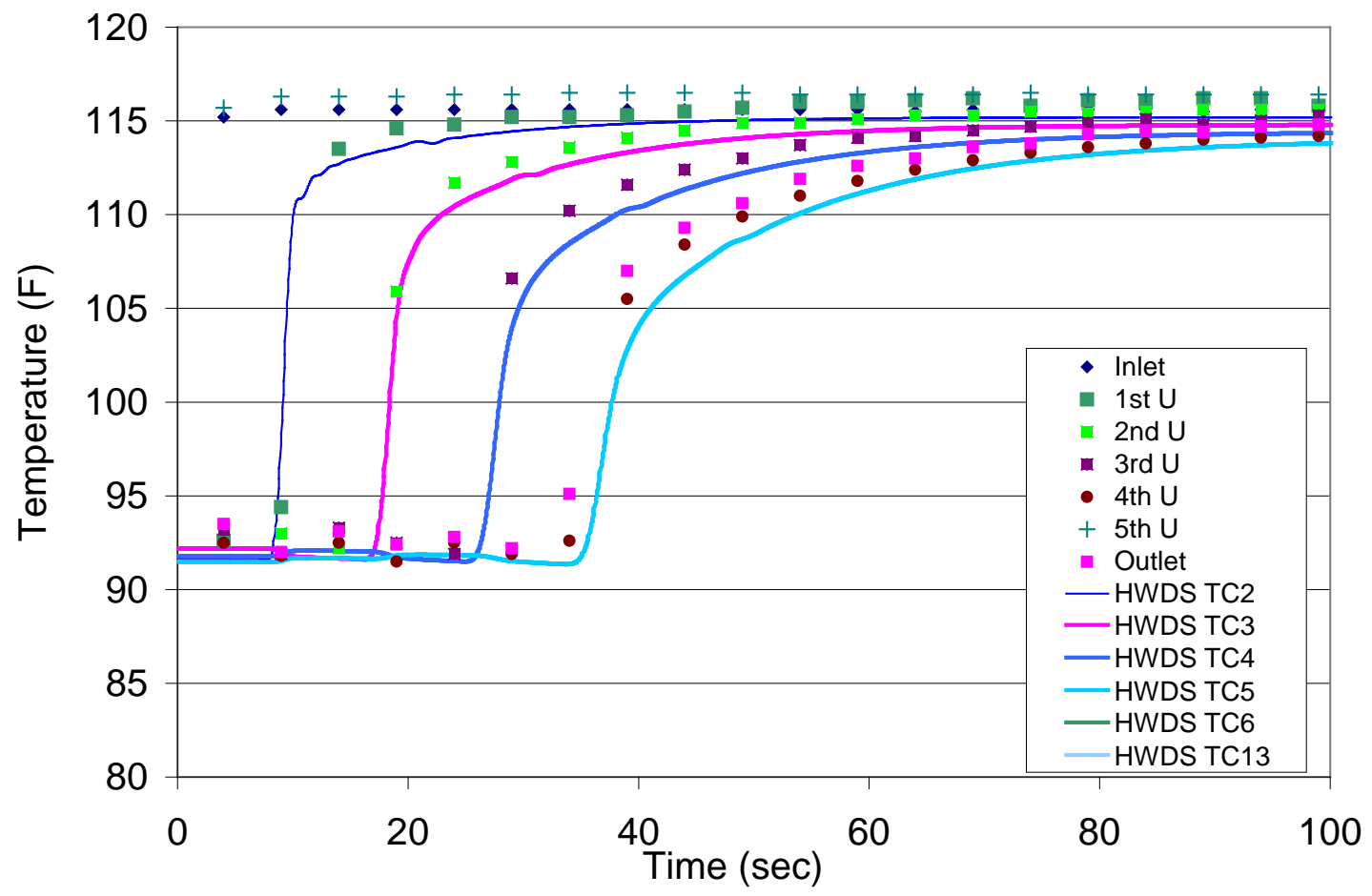




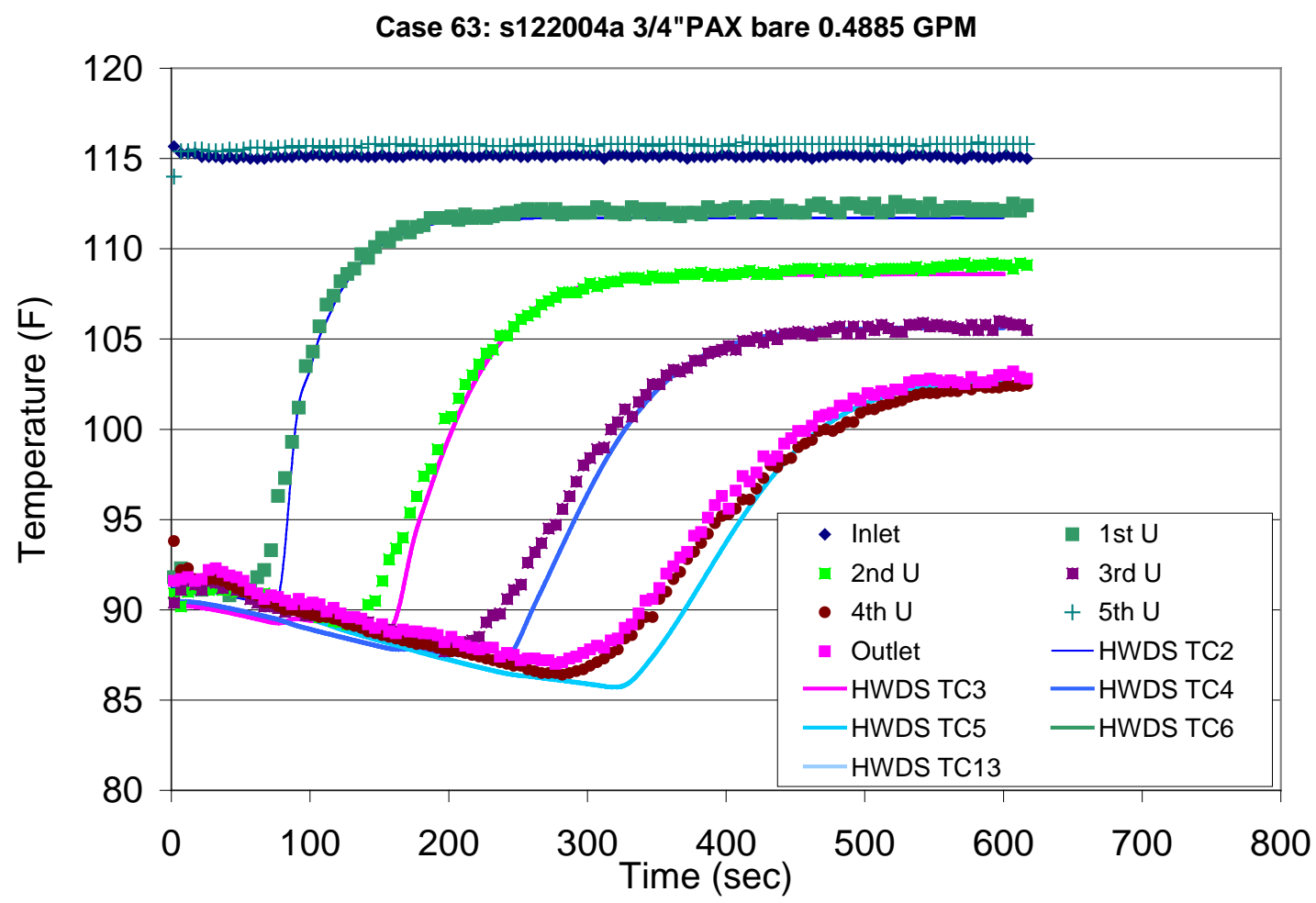

Case 64: s122004a 3/4"PAX bare 3.1455 GPM

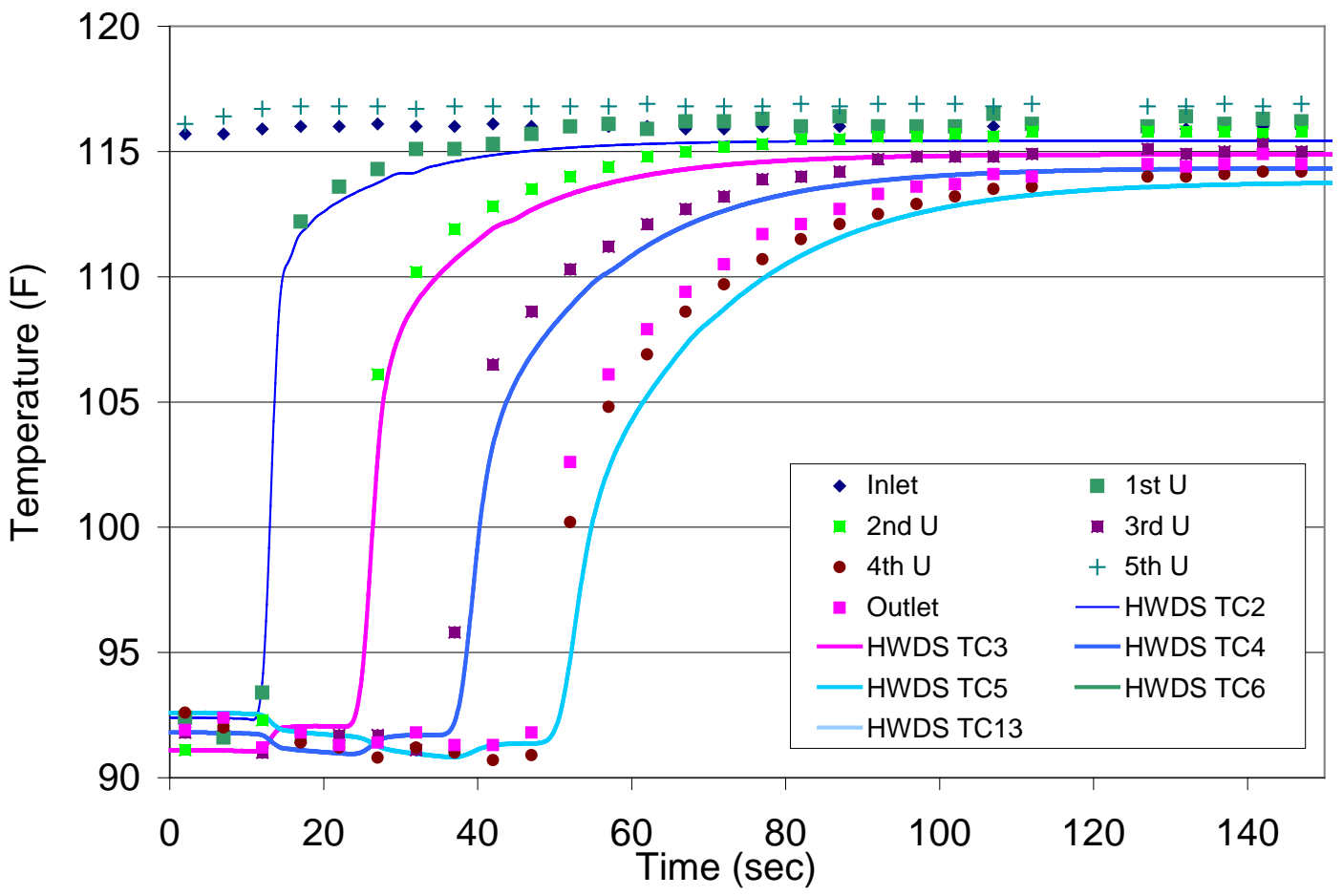




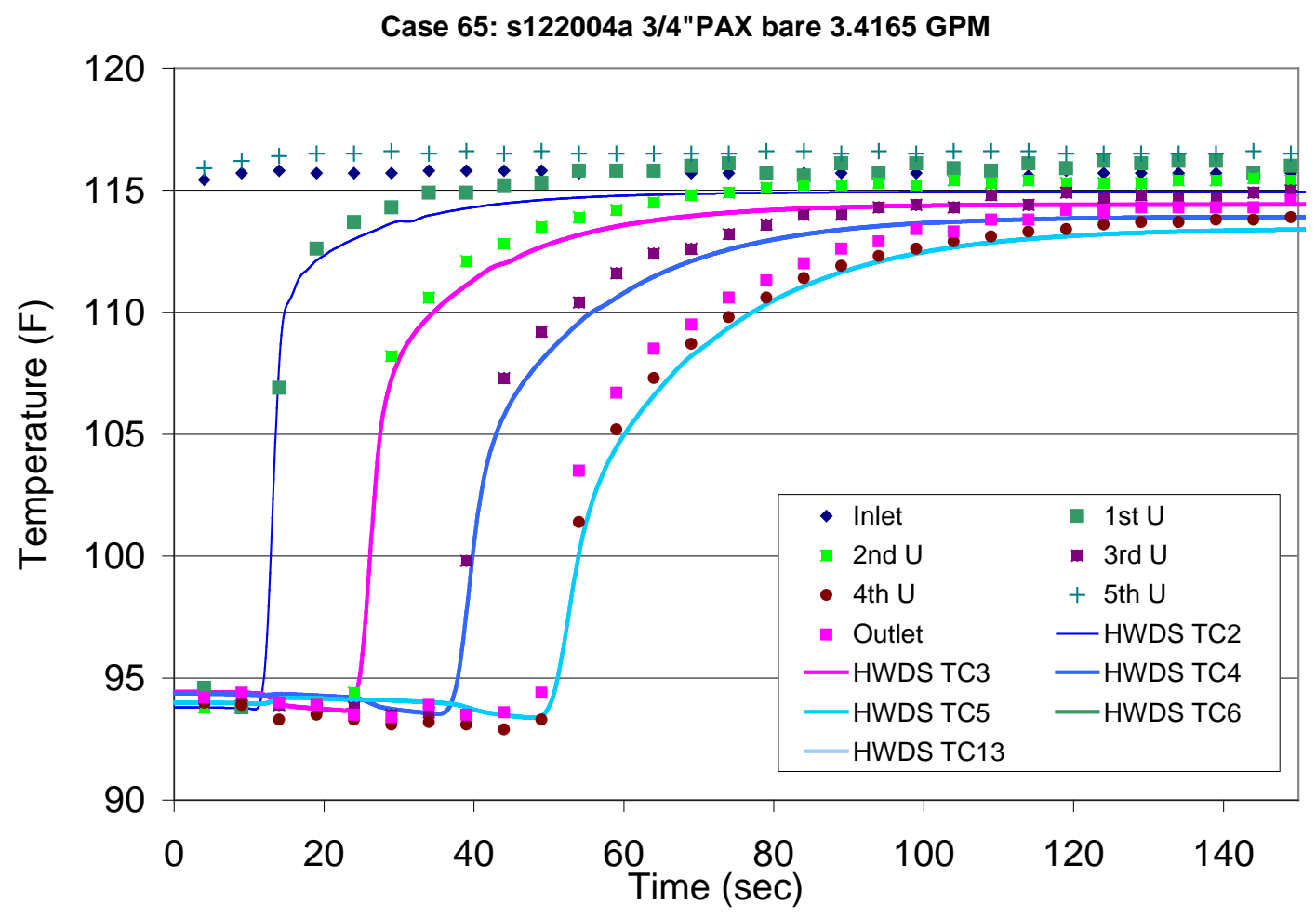

Case 66: s122004a 3/4"PAX bare 0.8991 GPM

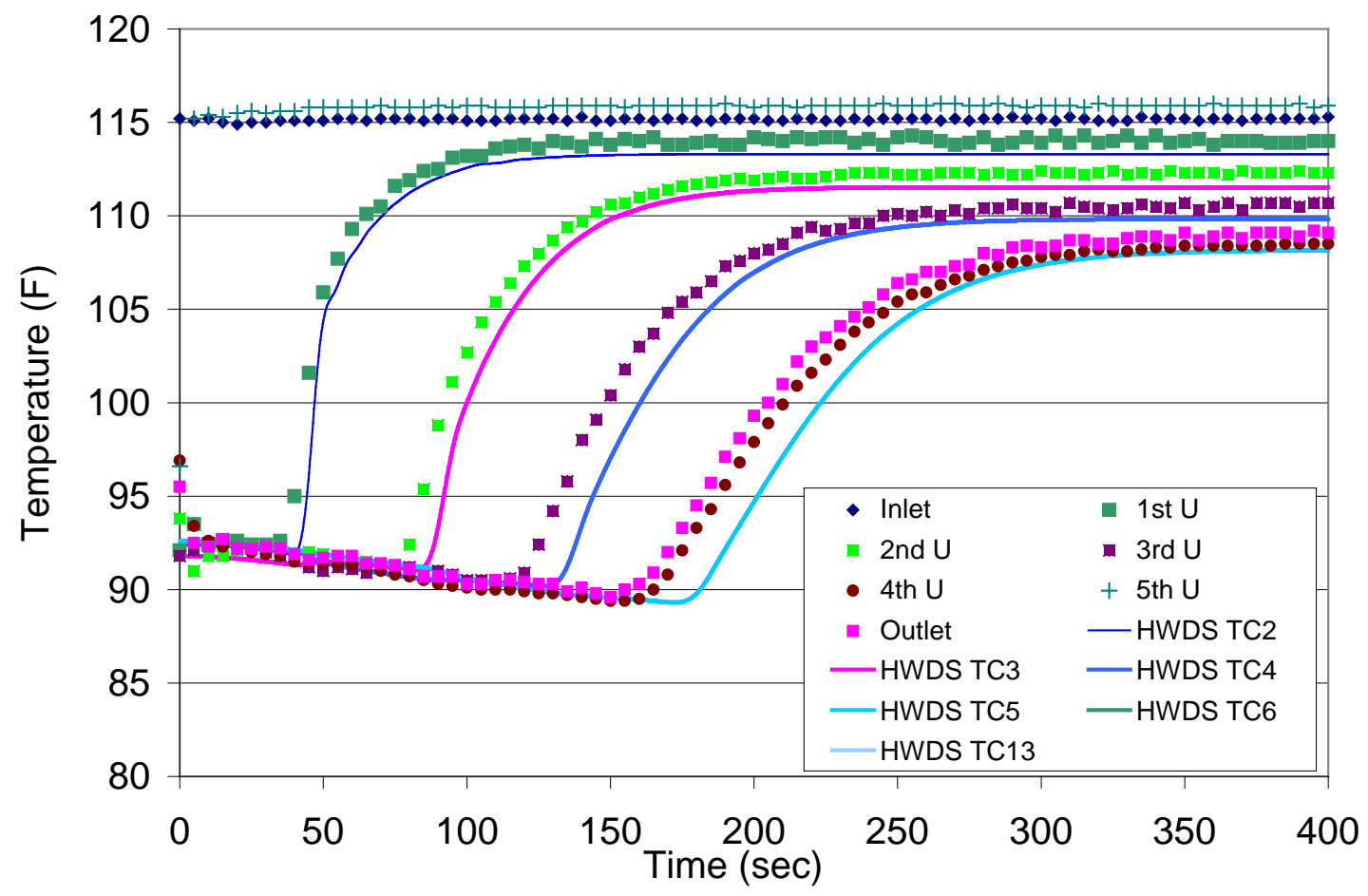



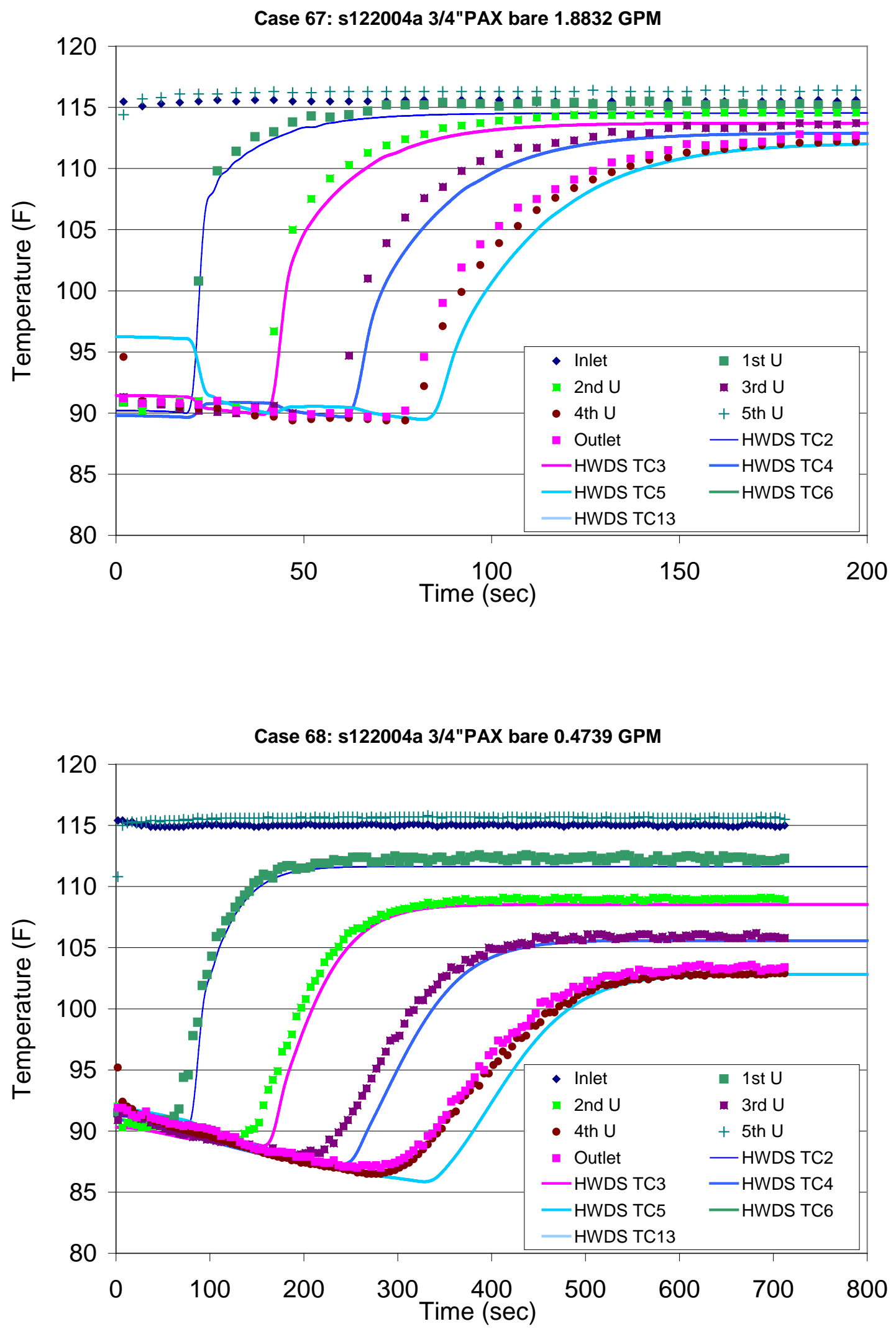


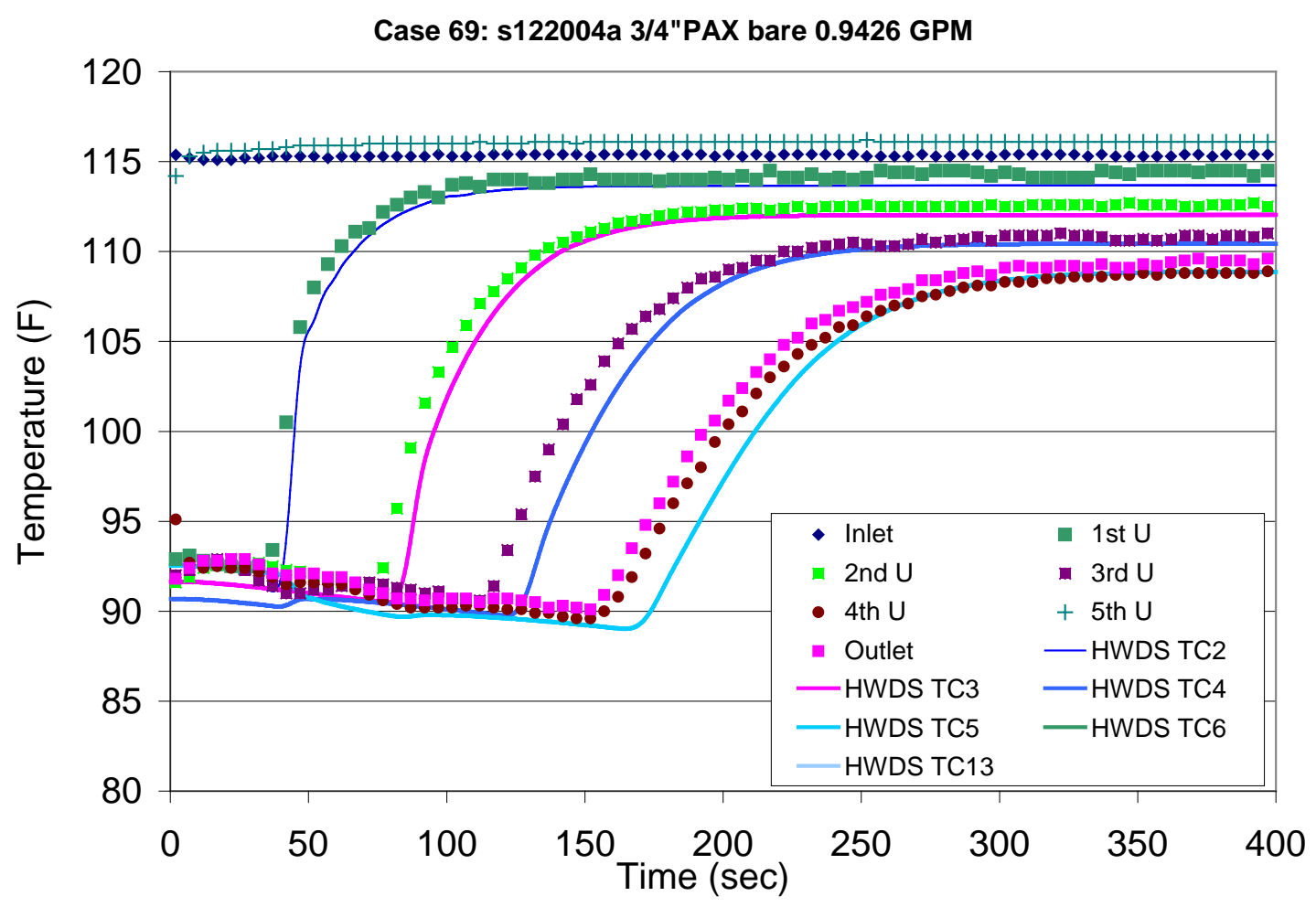

Case 70: s122004a 3/4"PAX bare 4.4109 GPM

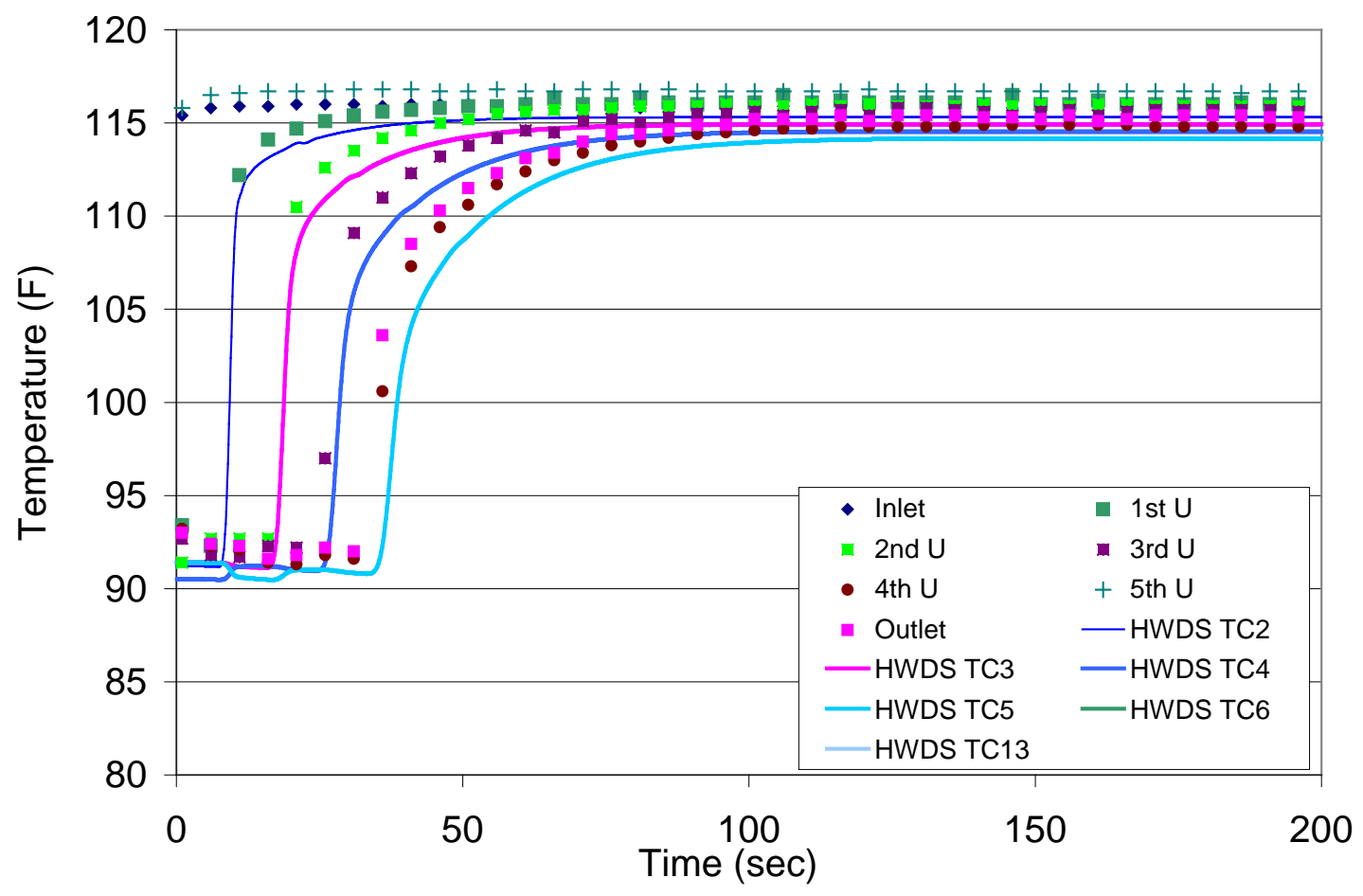



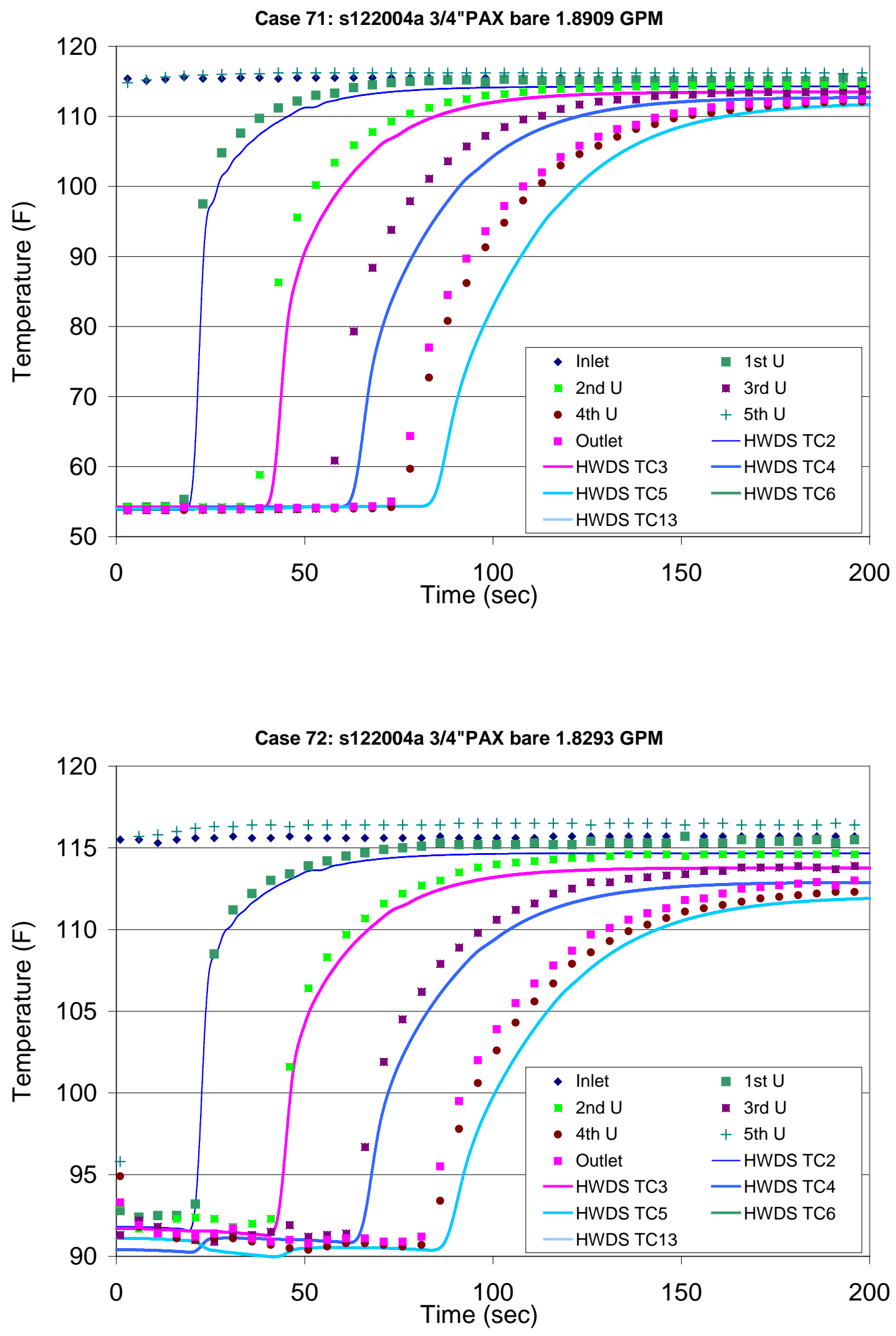

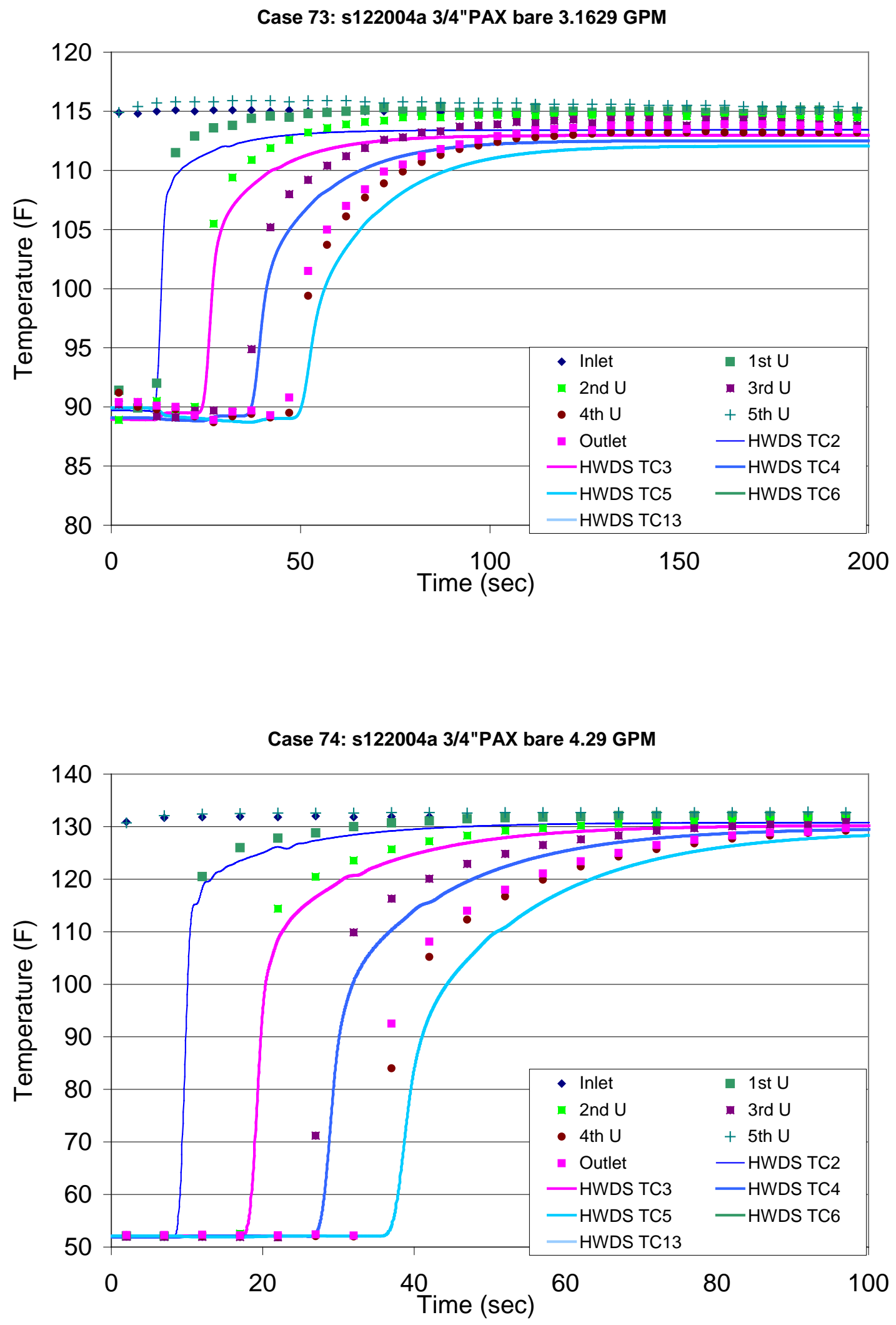


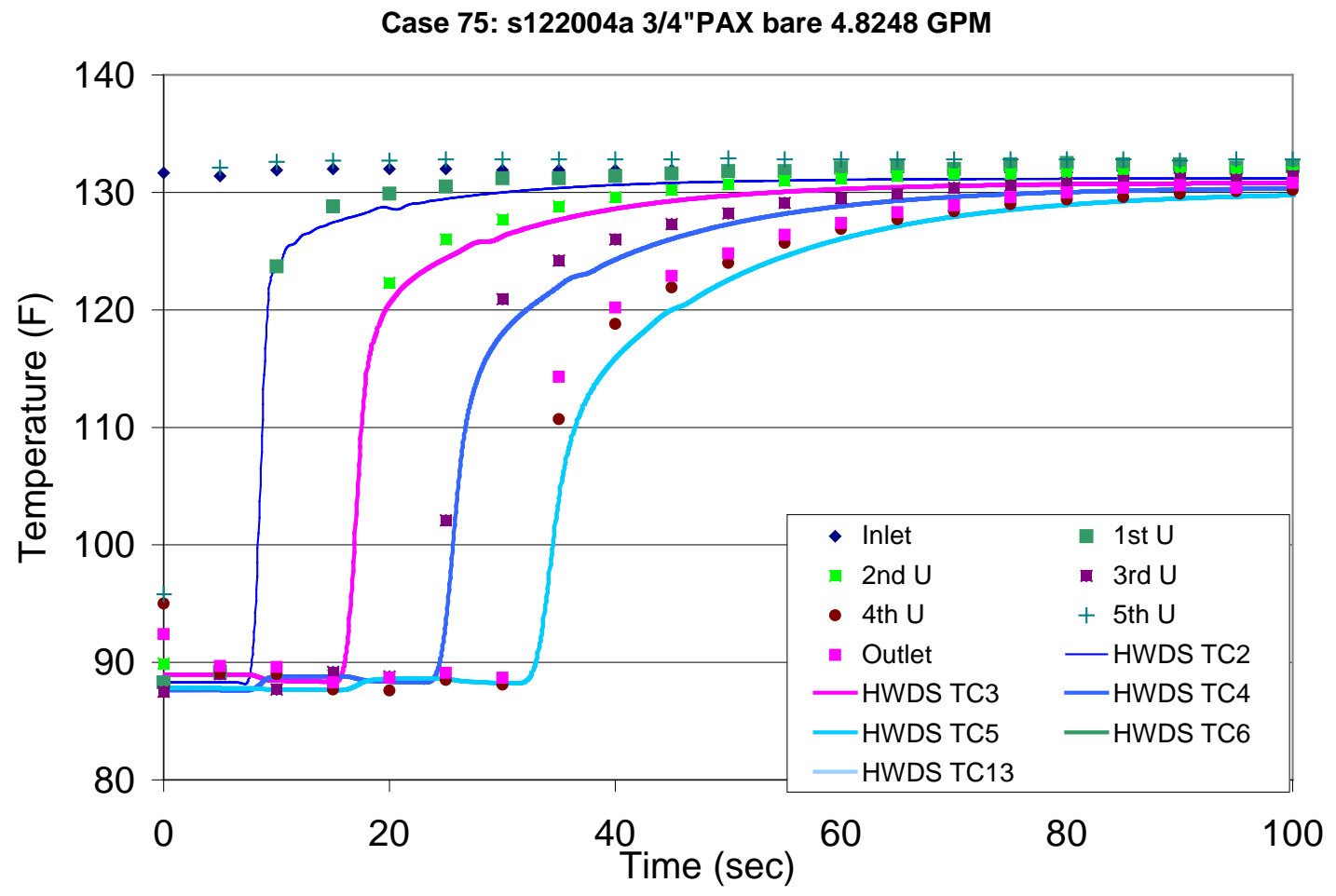

Case 76: s122004a 3/4"PAX bare 0.5244 GPM

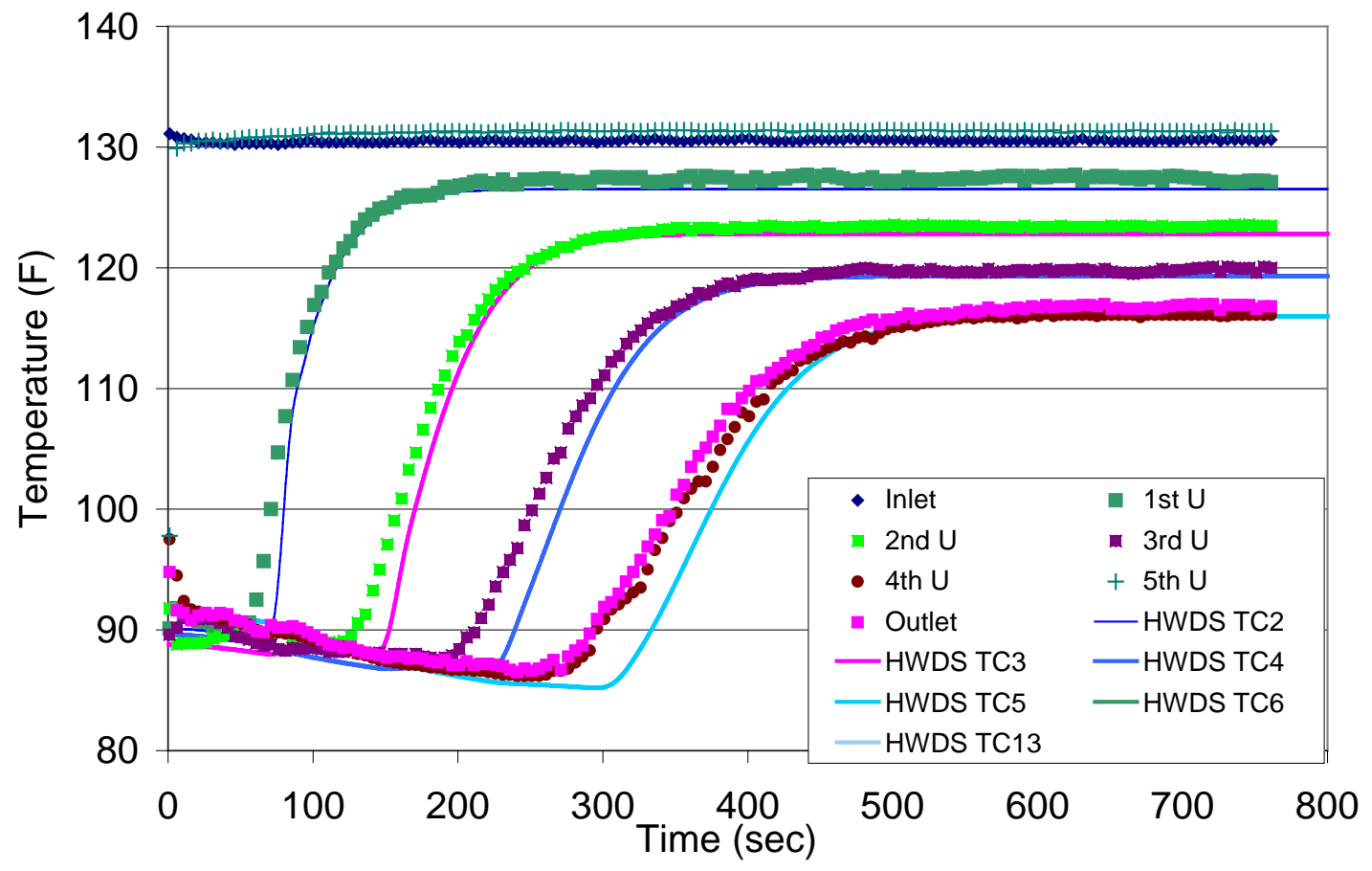




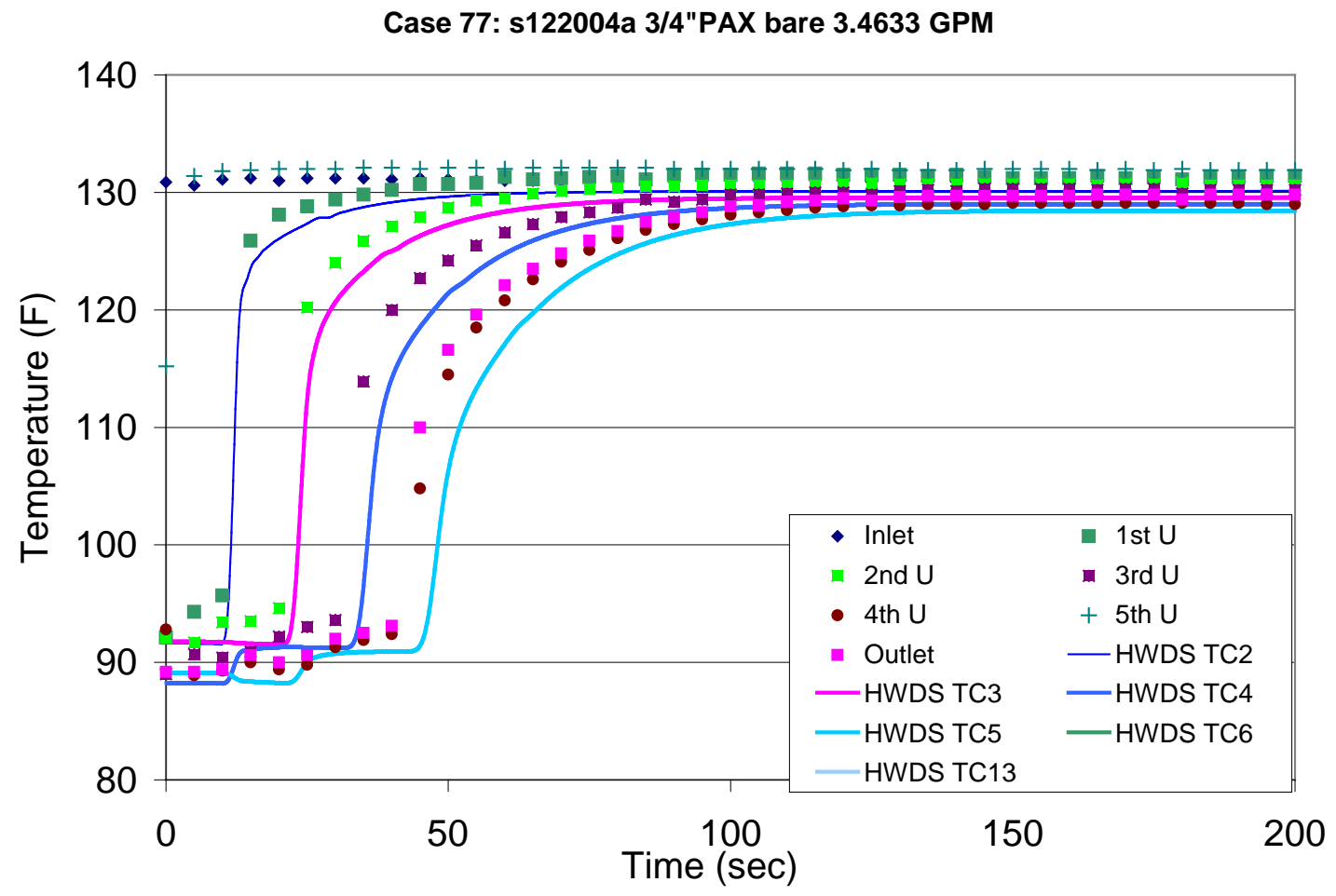

Case 78: s122004a 3/4"PAX bare 2.1561 GPM

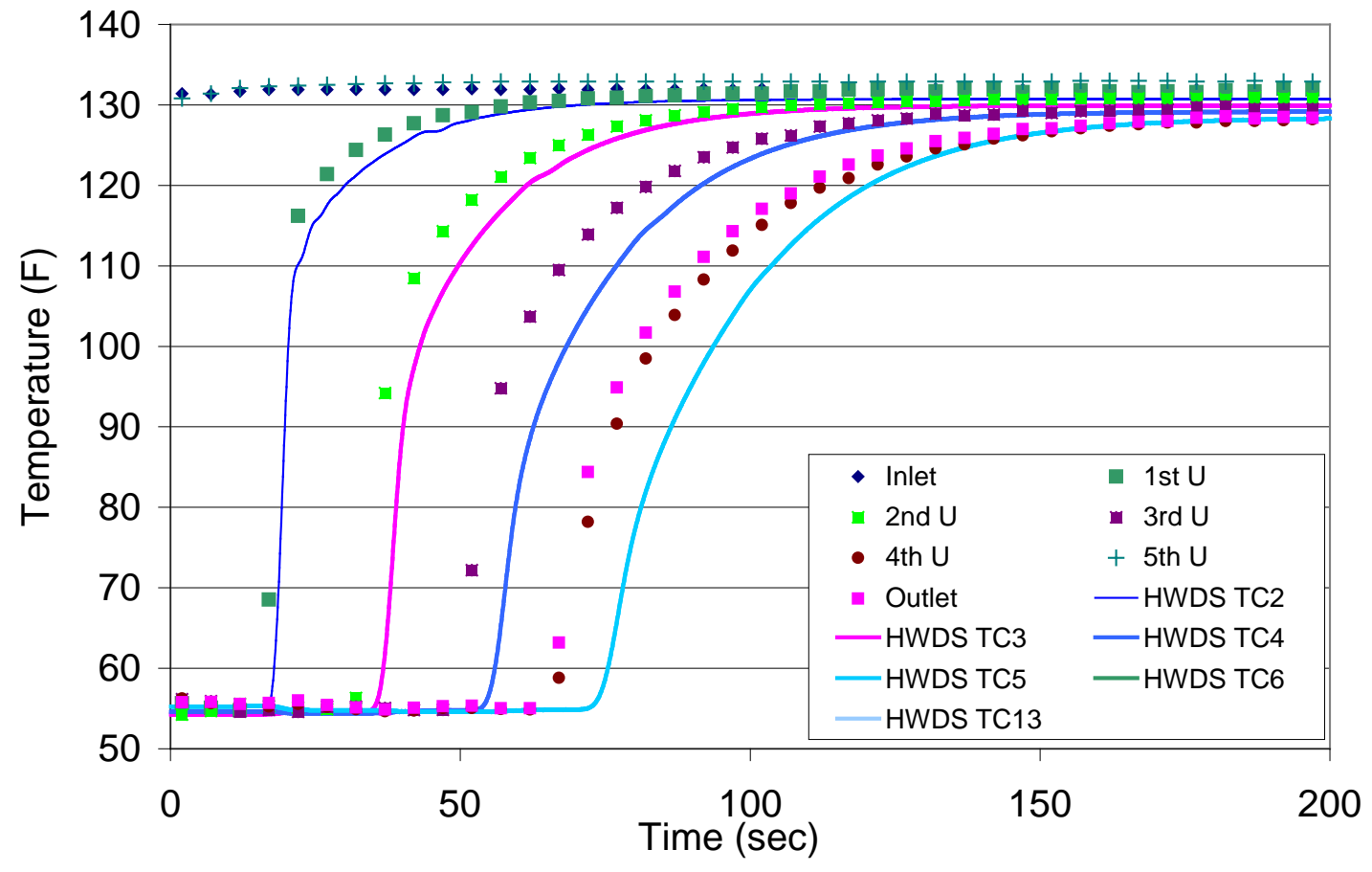




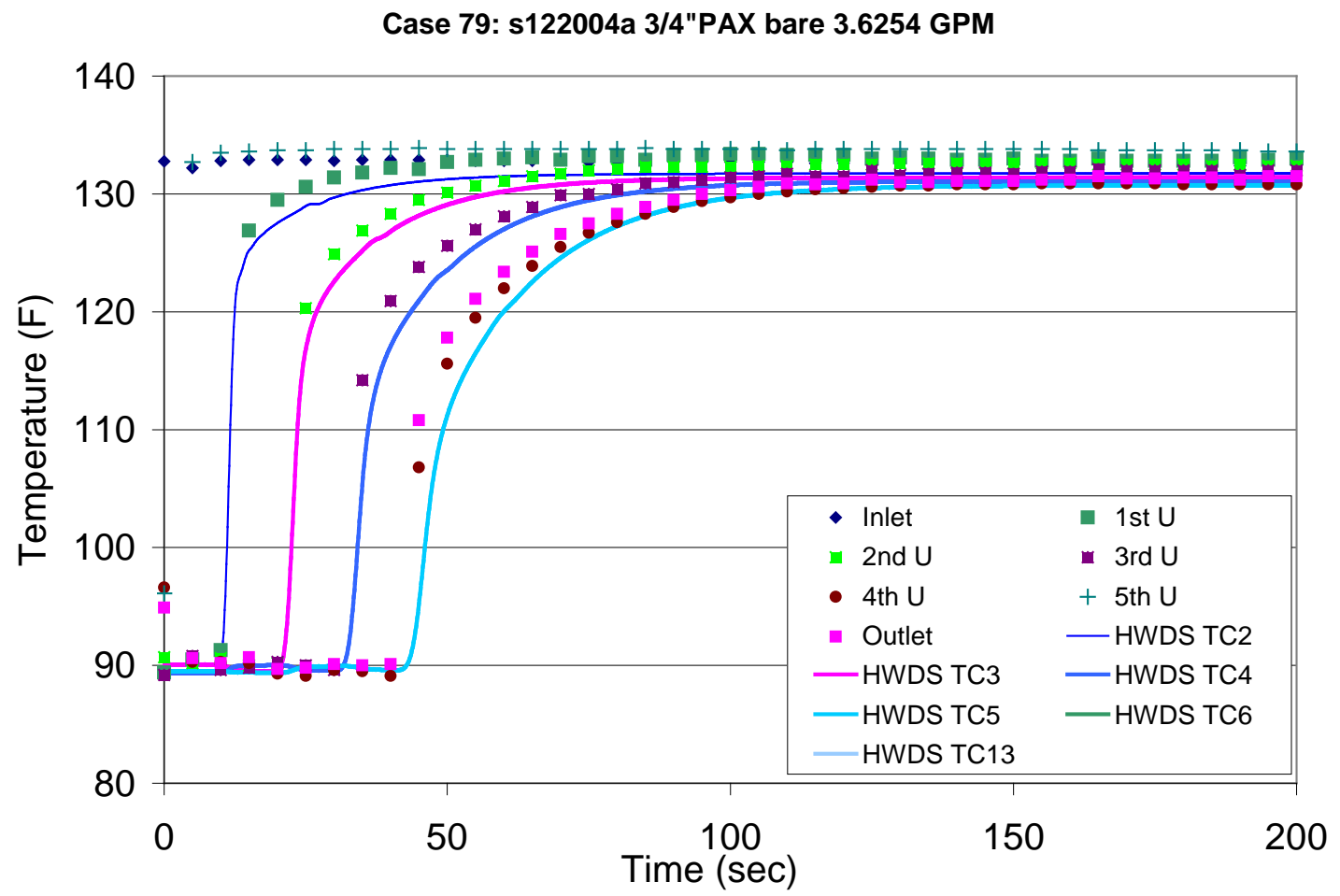

Case 80: s122004a 3/4"PAX bare 0.9738 GPM

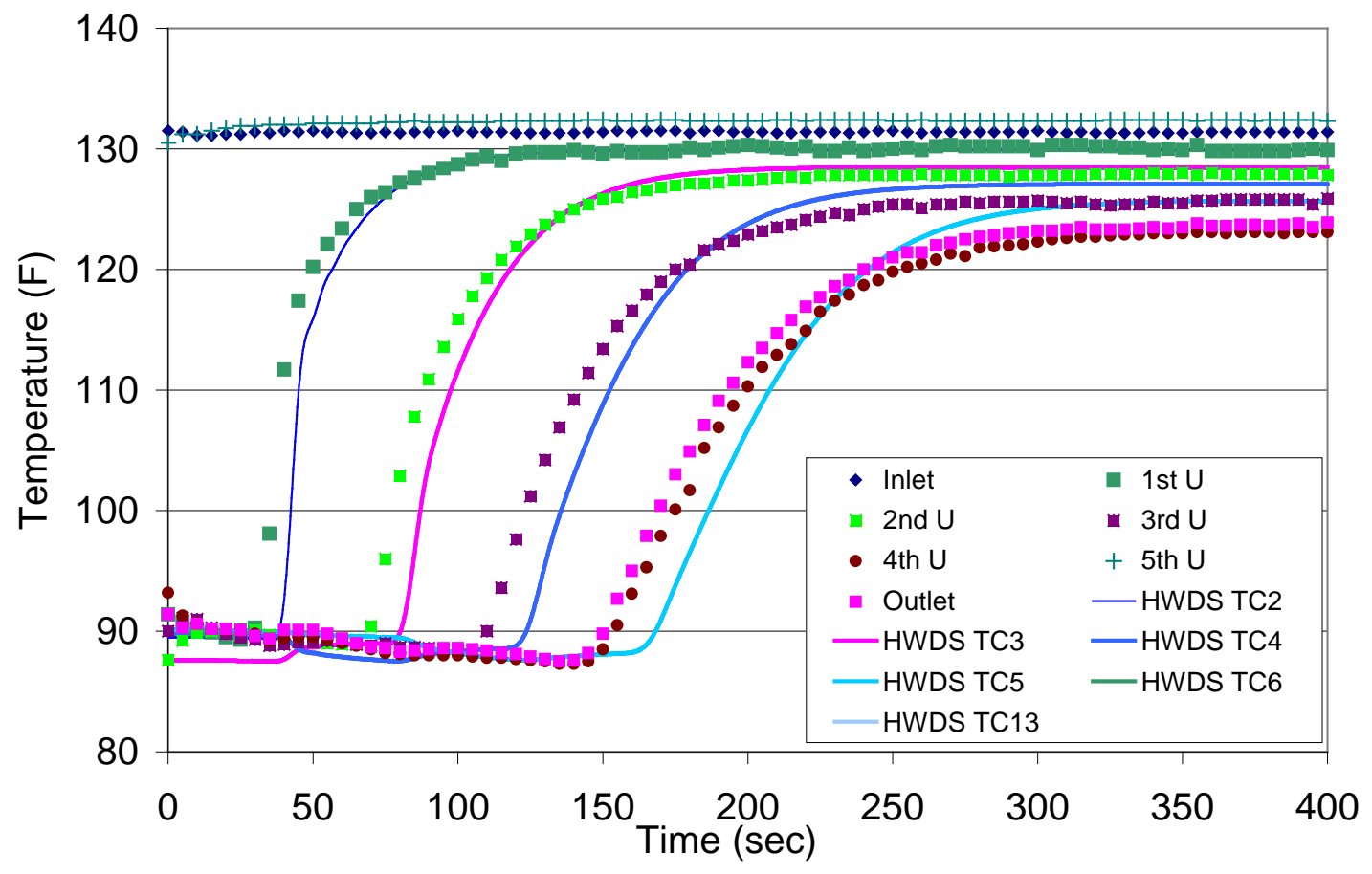




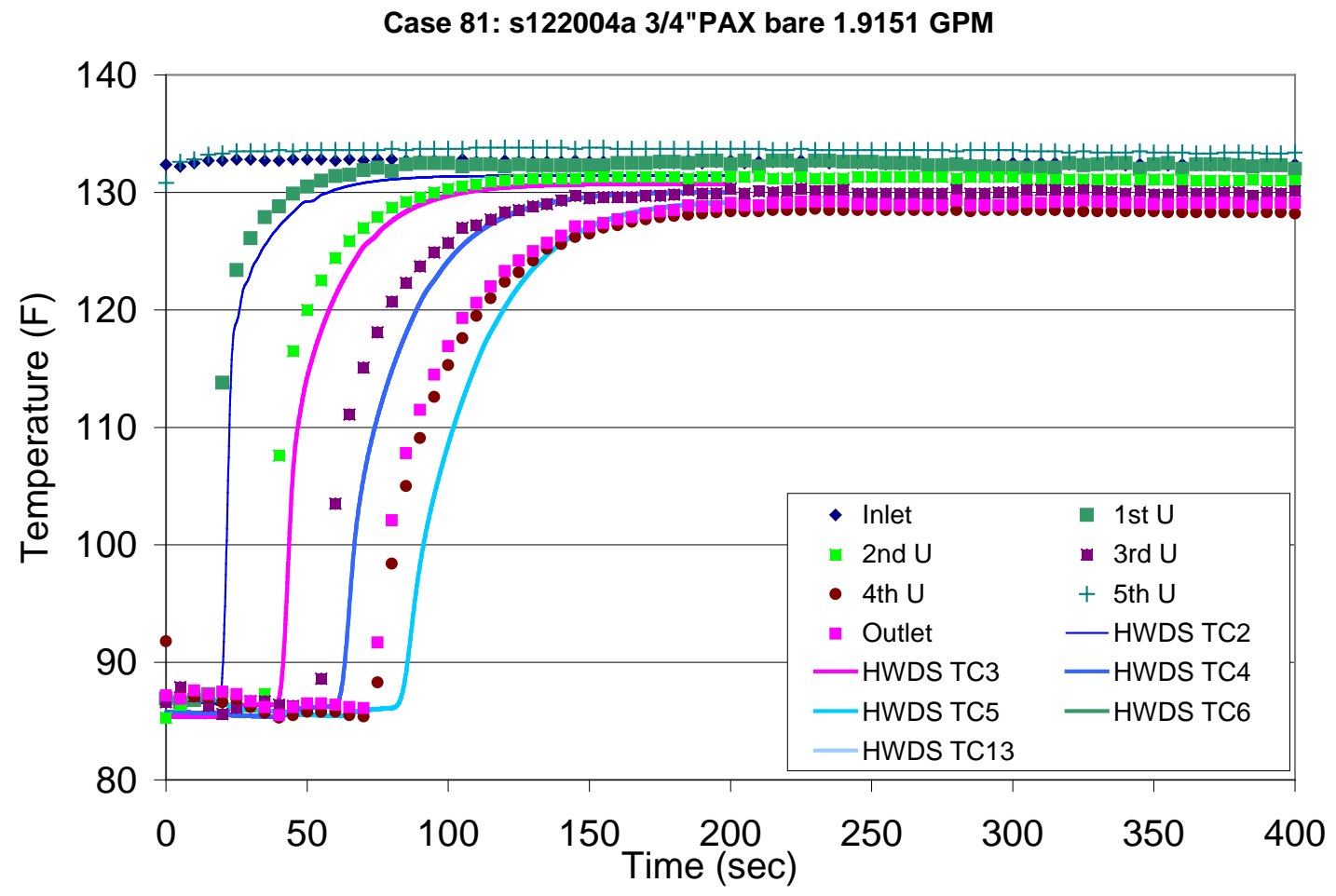

Case 82: s122004a 3/4"PAX bare 0.5272 GPM

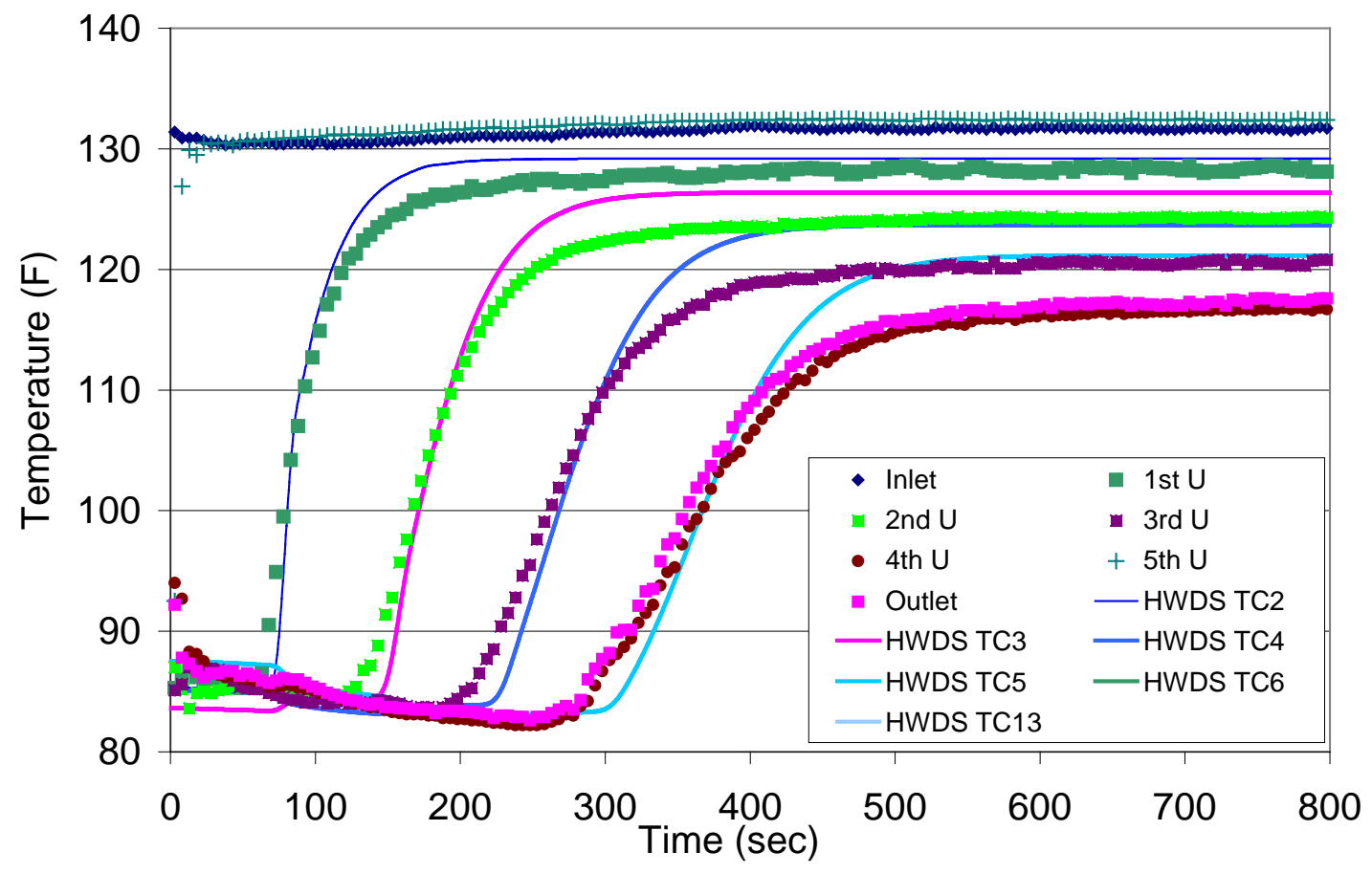




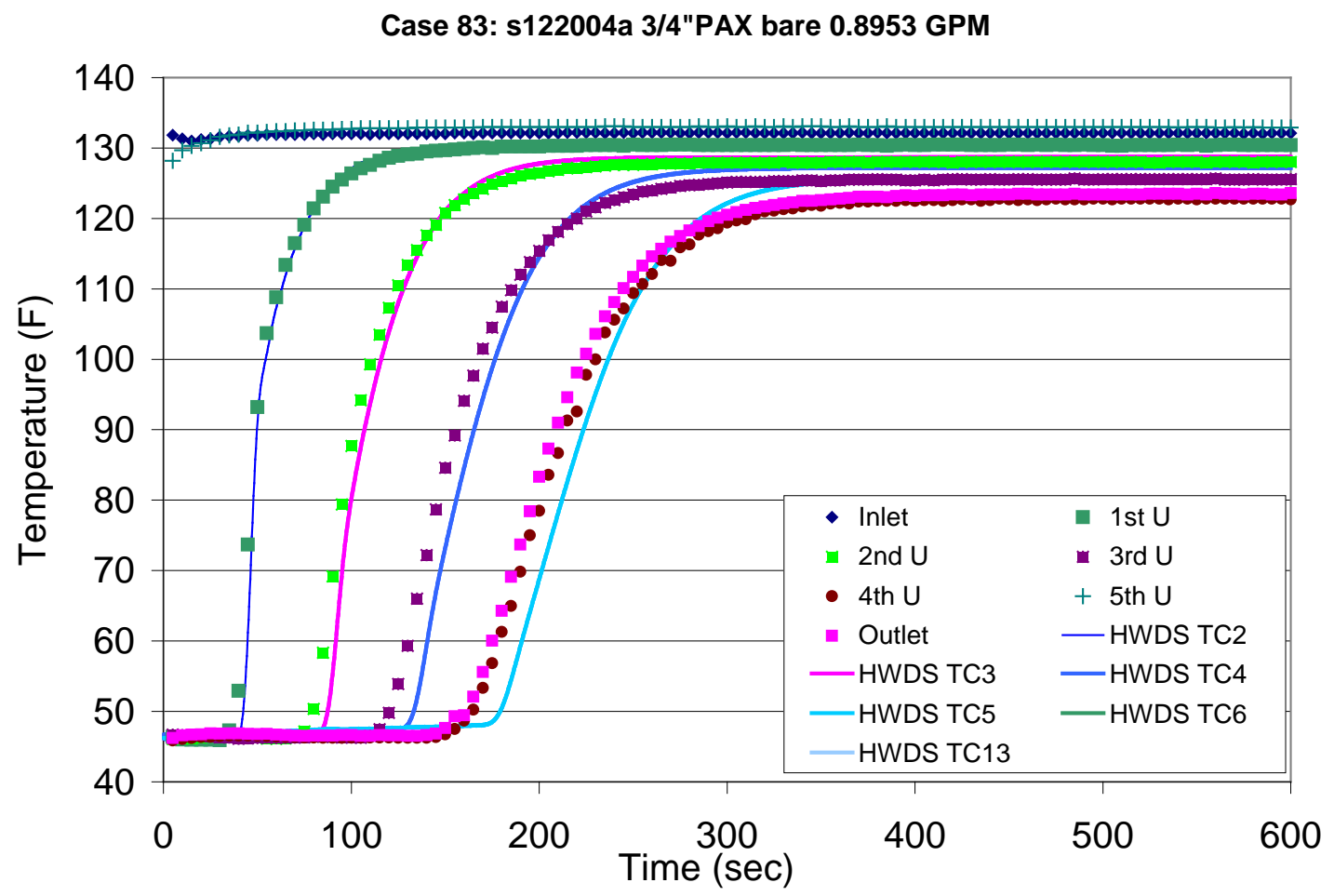

Case 84: s122004a 3/4"PAX bare 3.087GPM

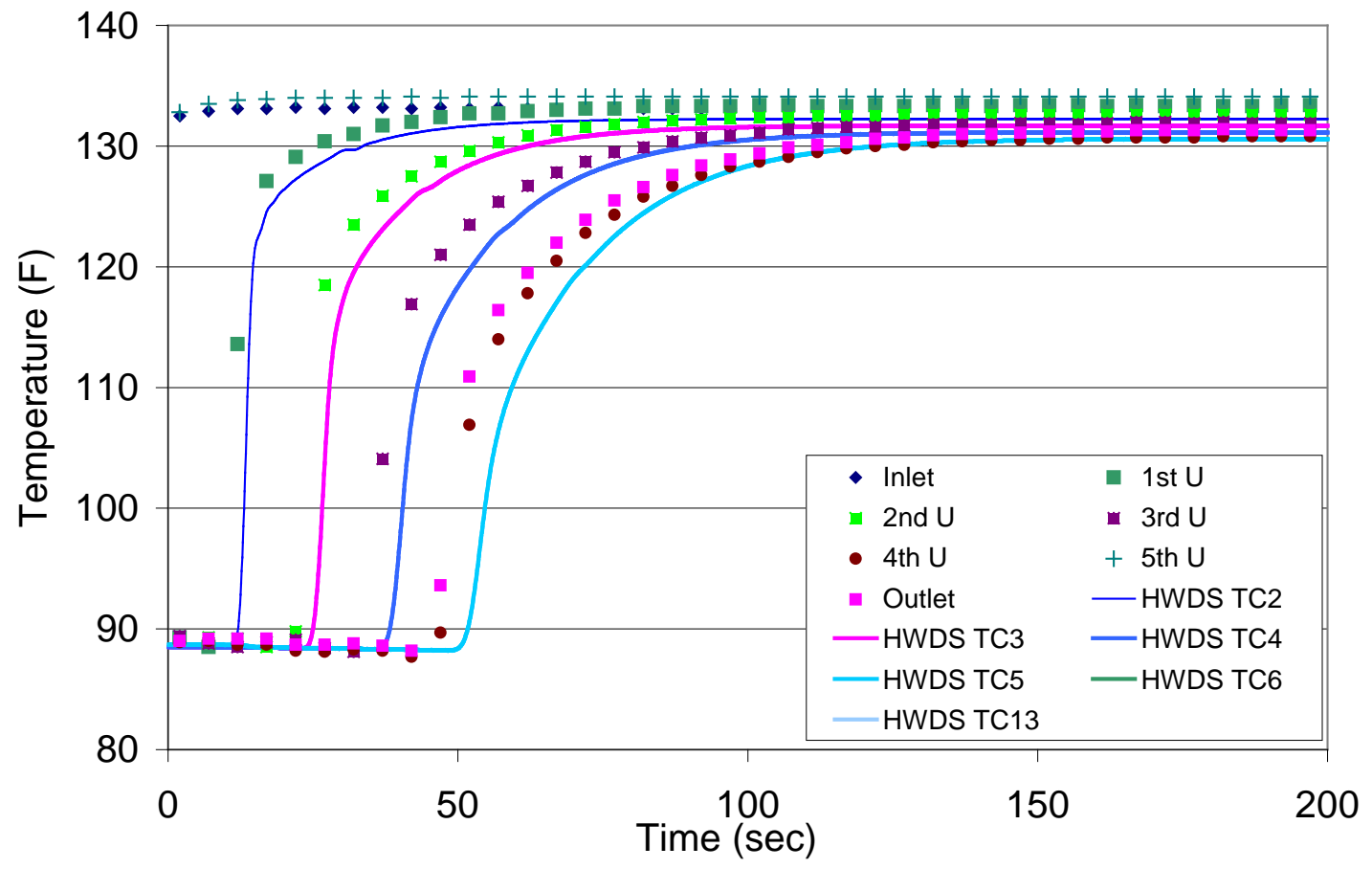




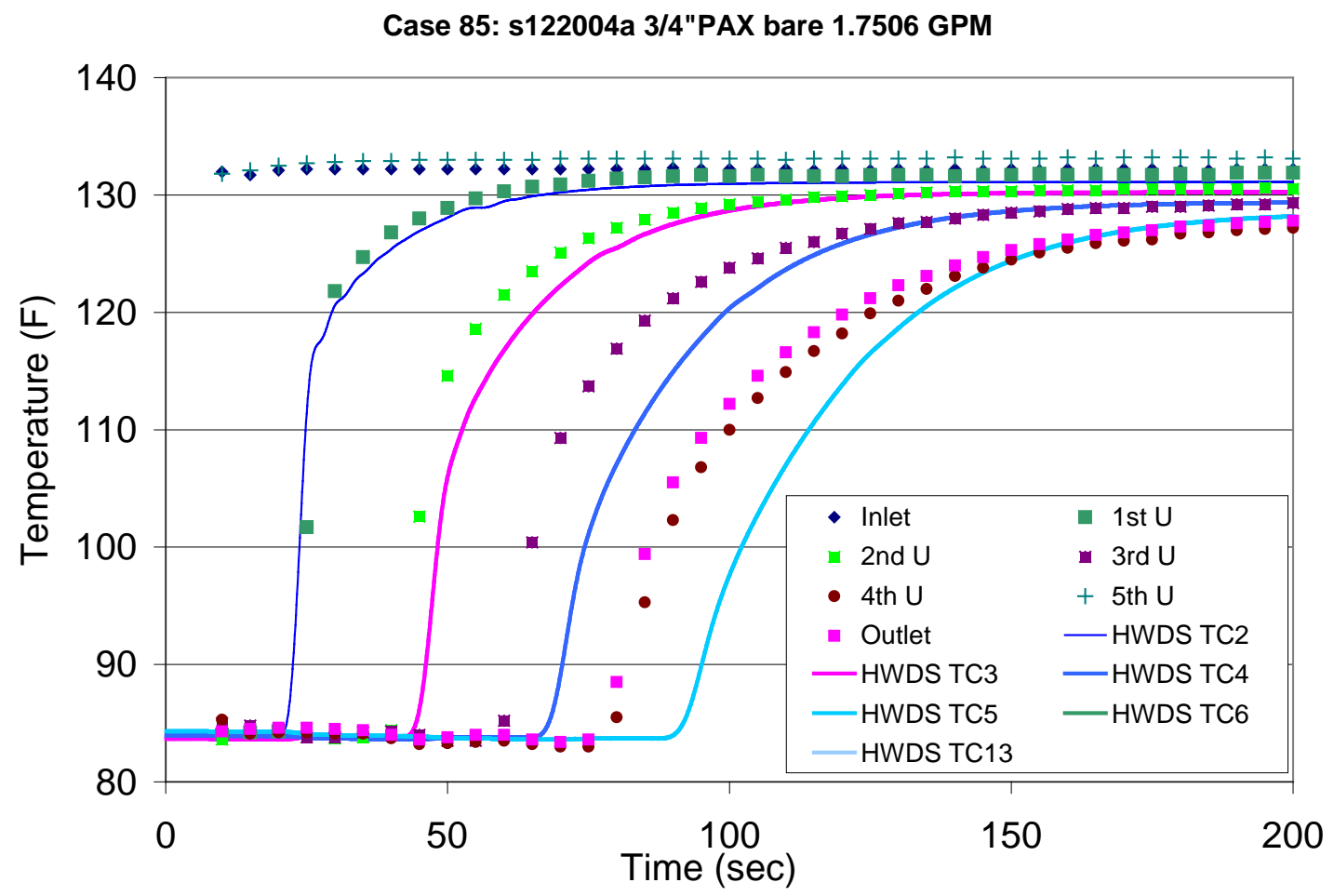

Case 86: s122004a 3/4"PAX bare 0.9857 GPM

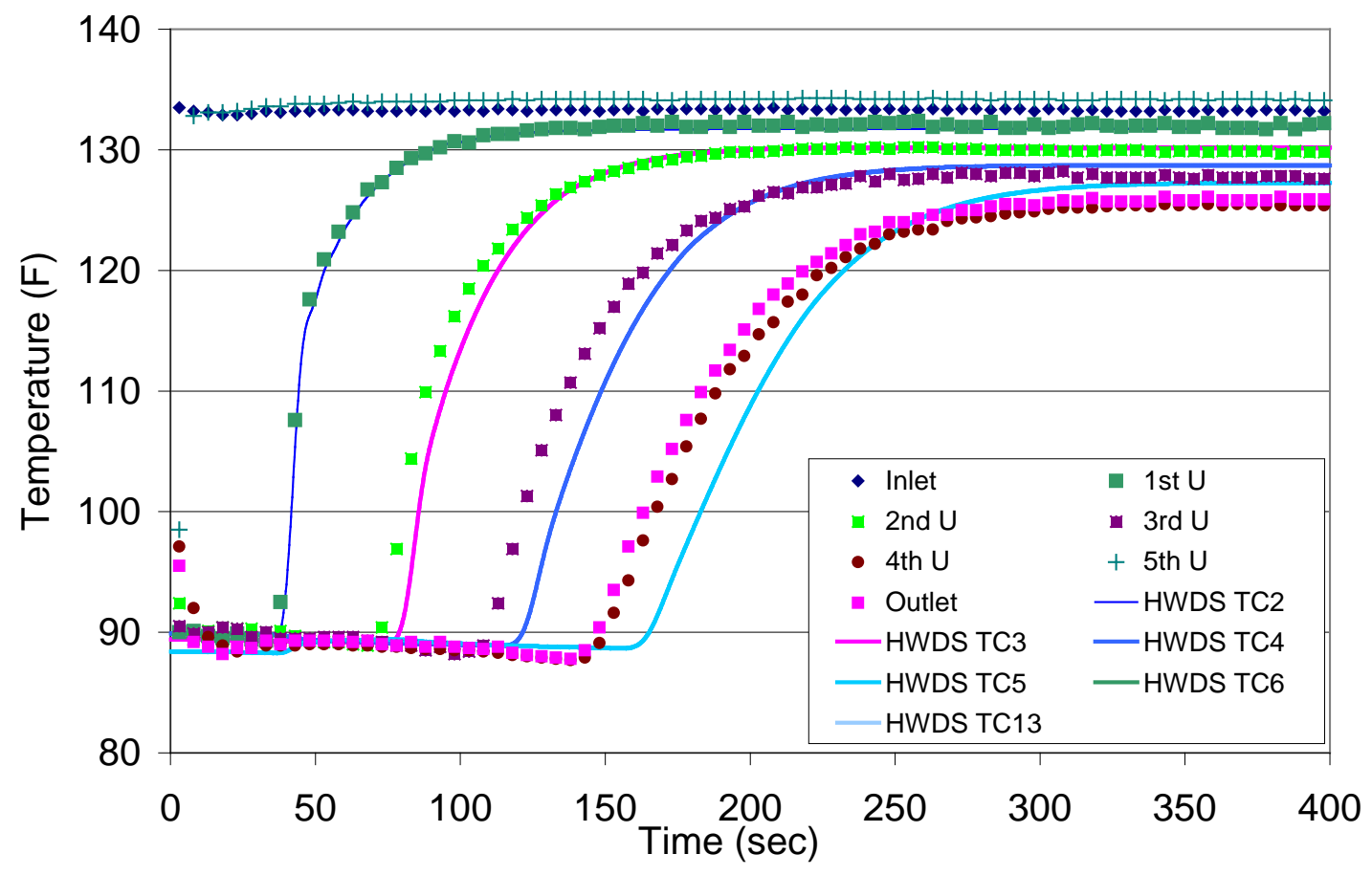




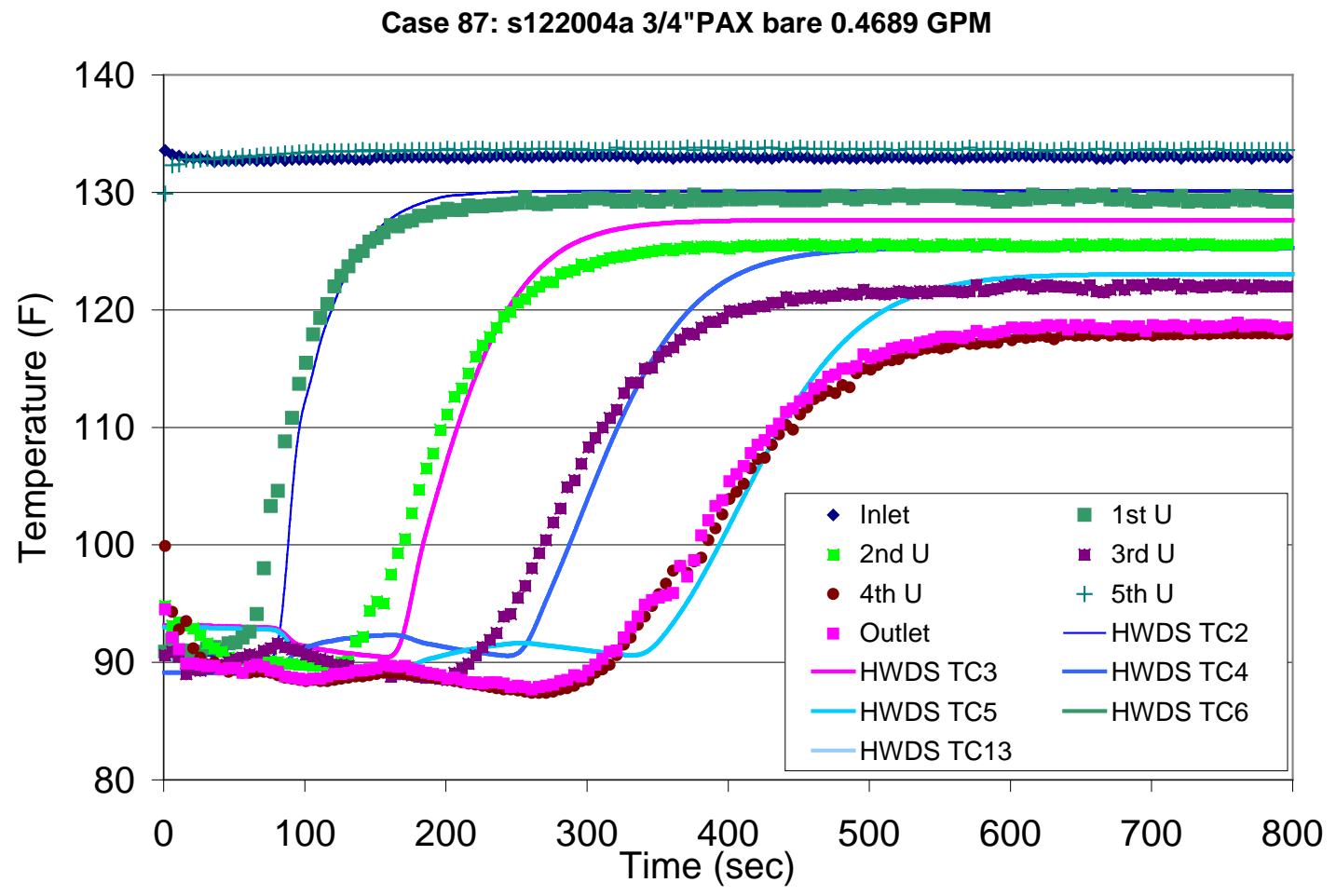

Case 88: s122004a 3/4"PAX bare 4.620 GPM

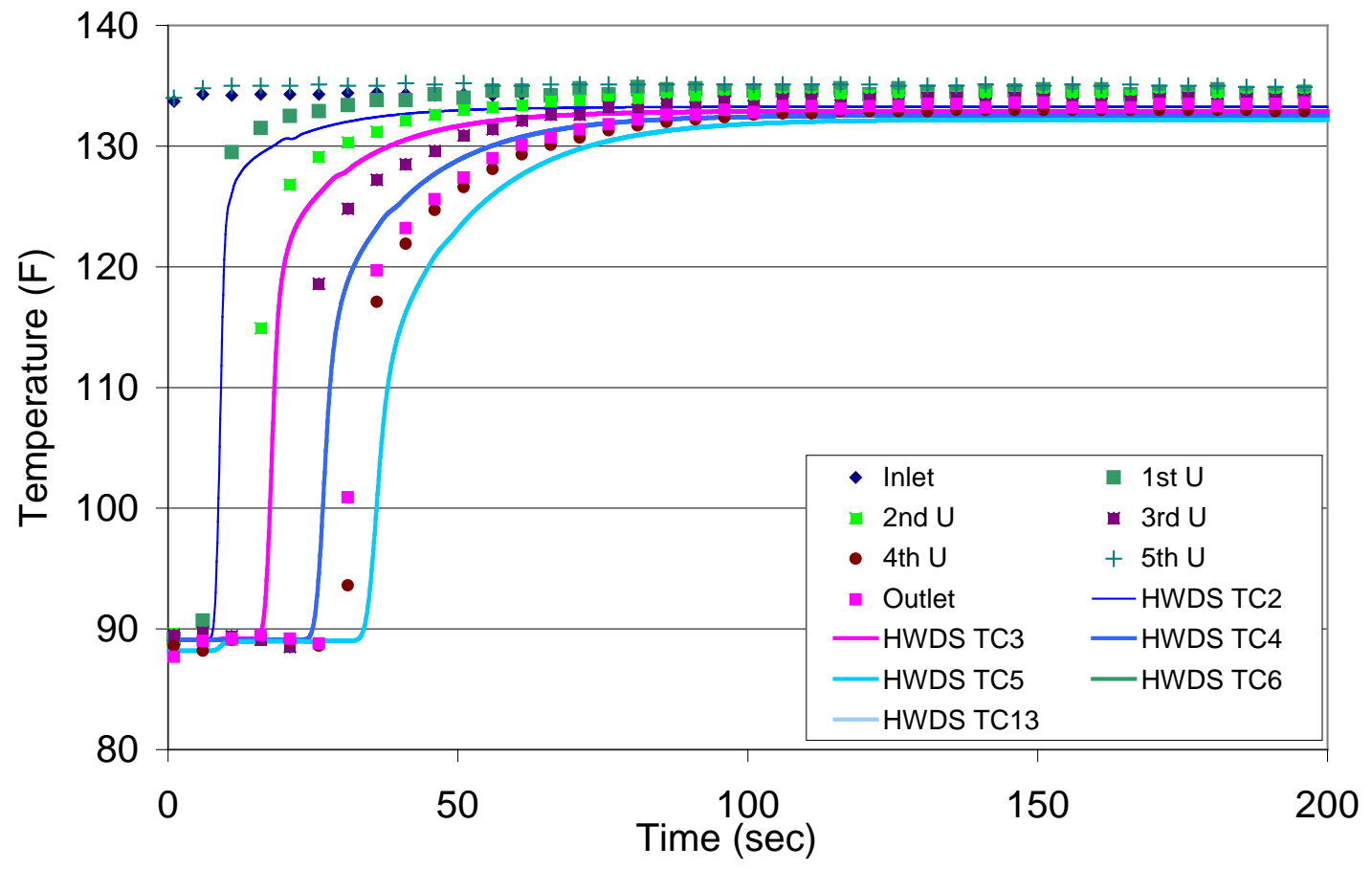




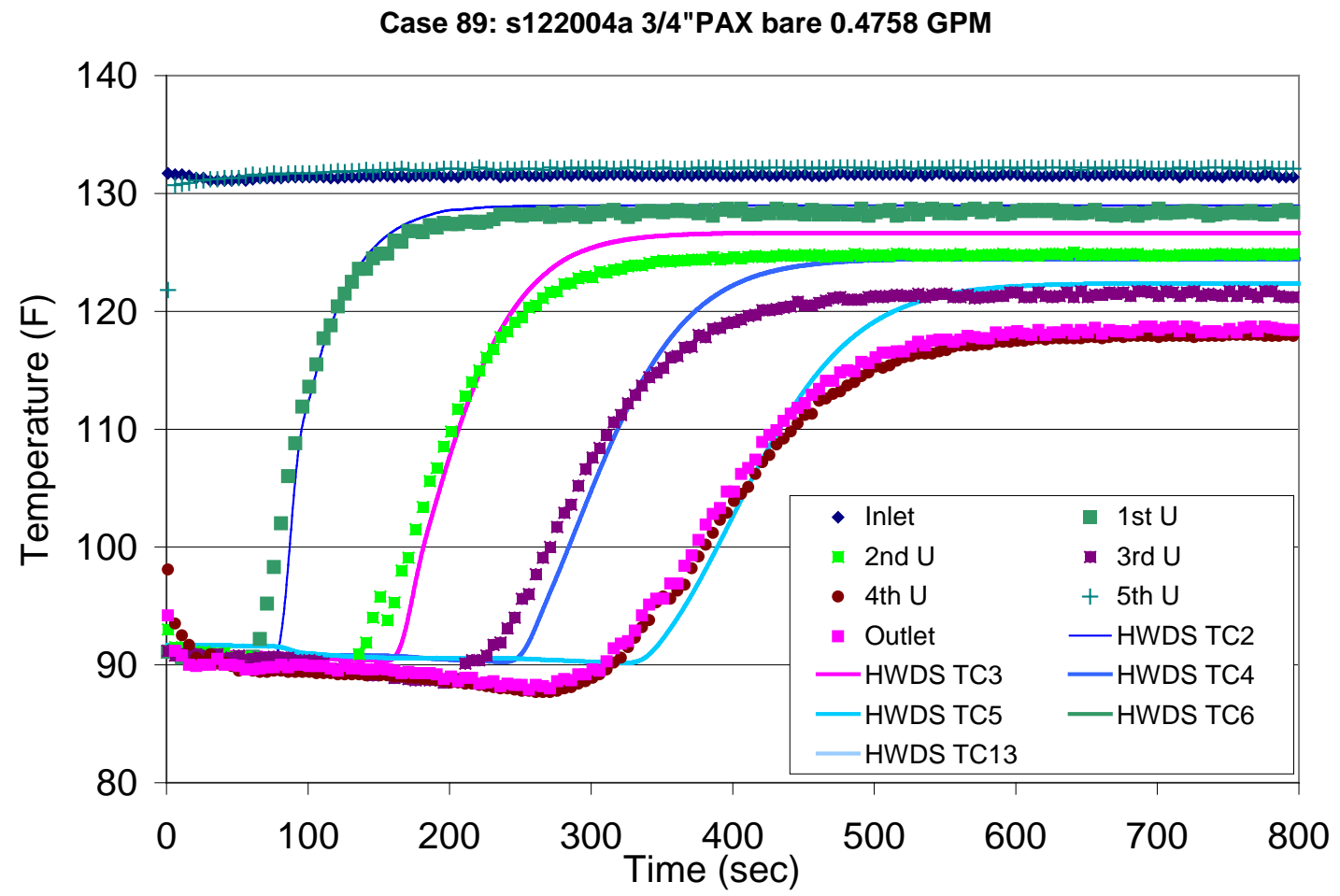

Case 90: s122004a 3/4"PAX bare 3.3115 GPM

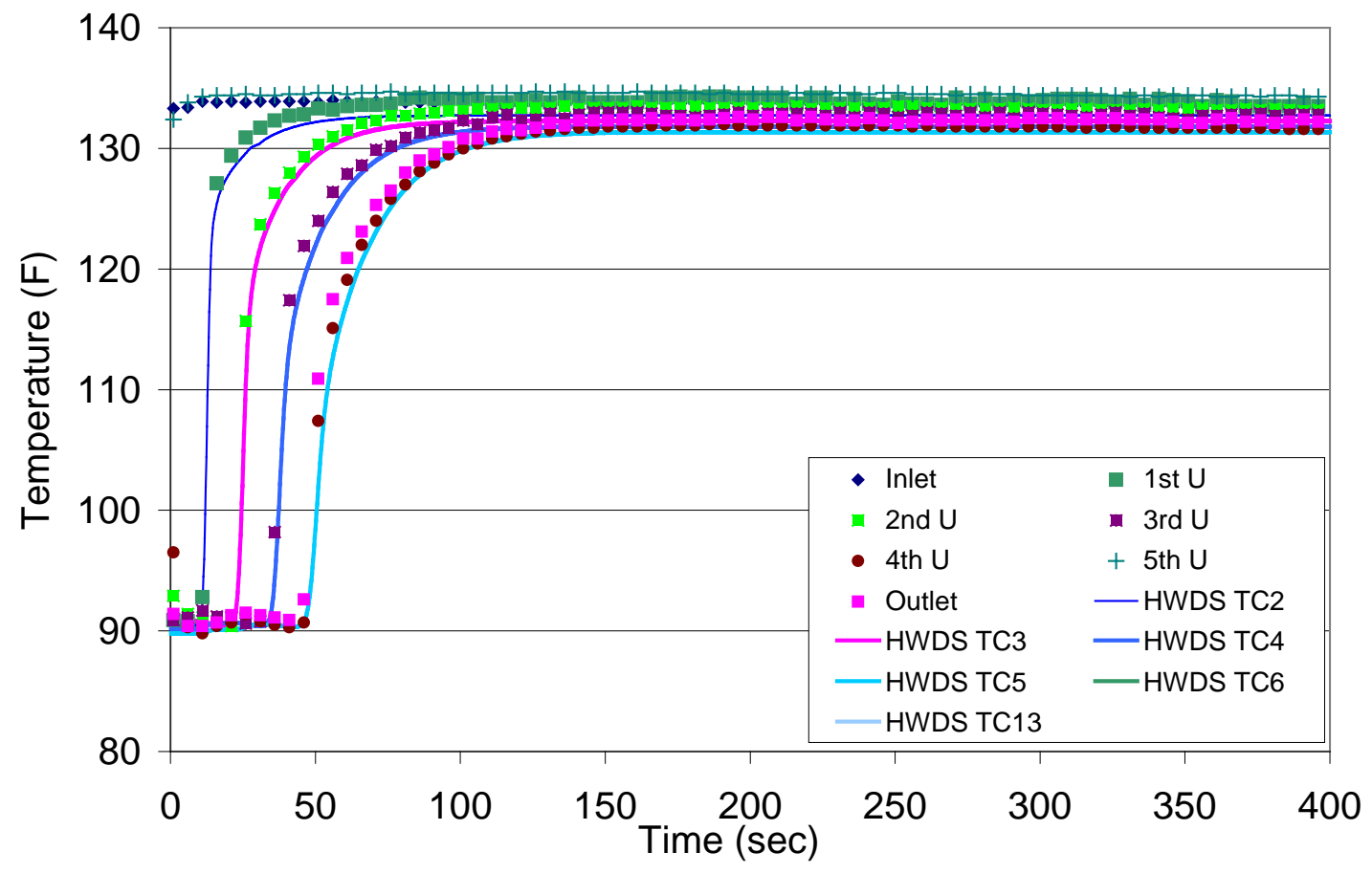




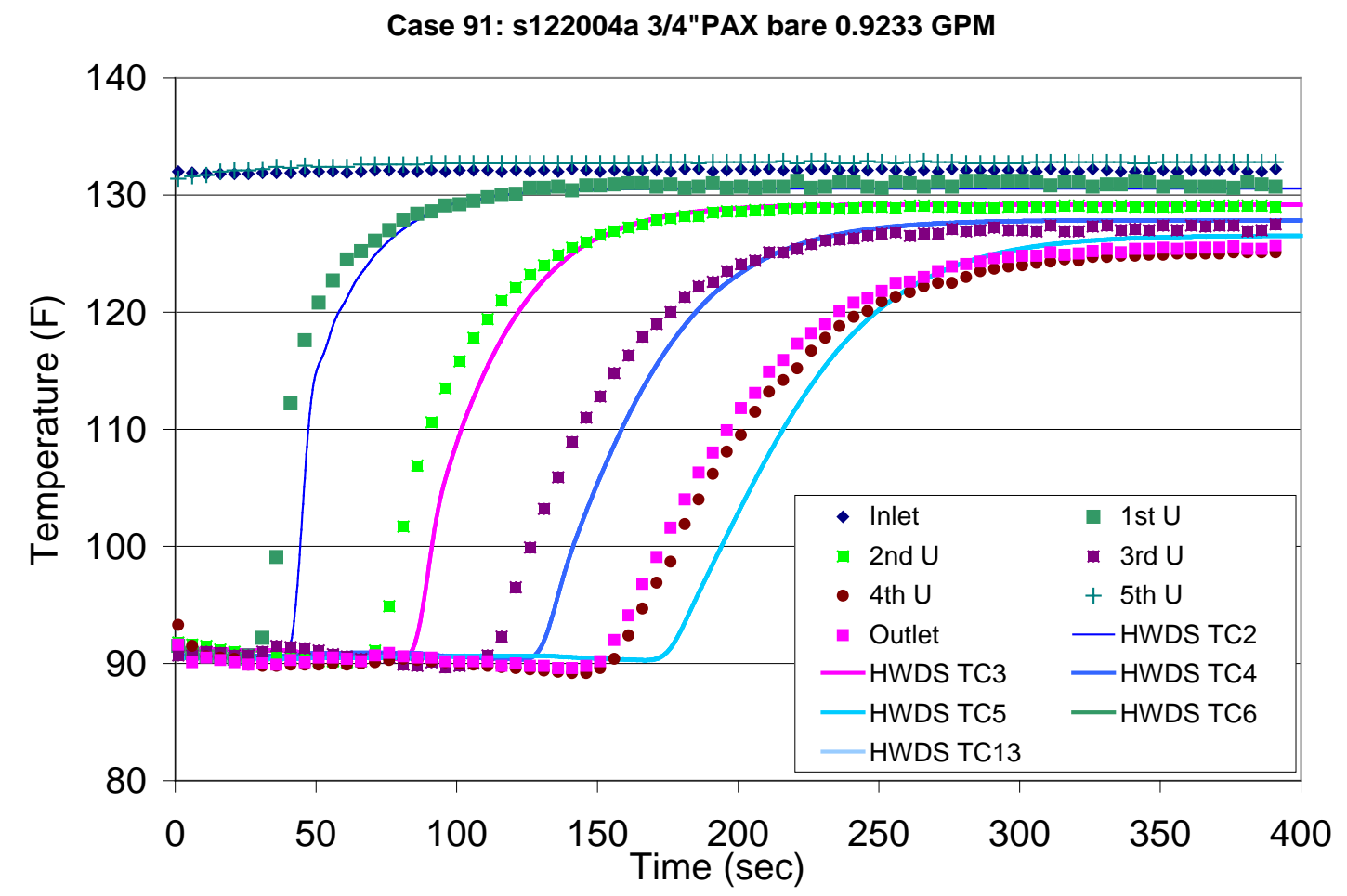

Case 92: s122004a 3/4"PAX bare 1.7838 GPM

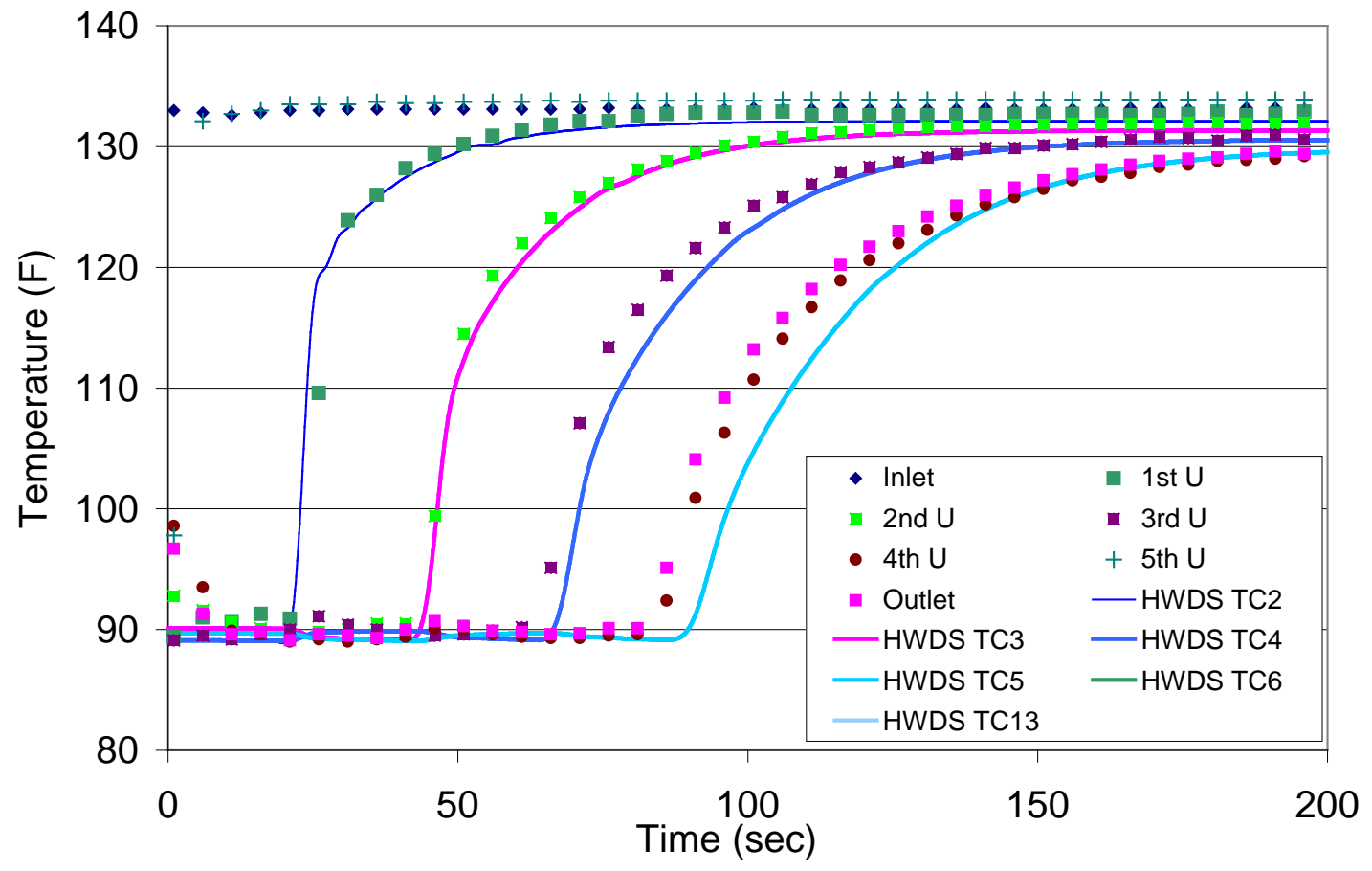



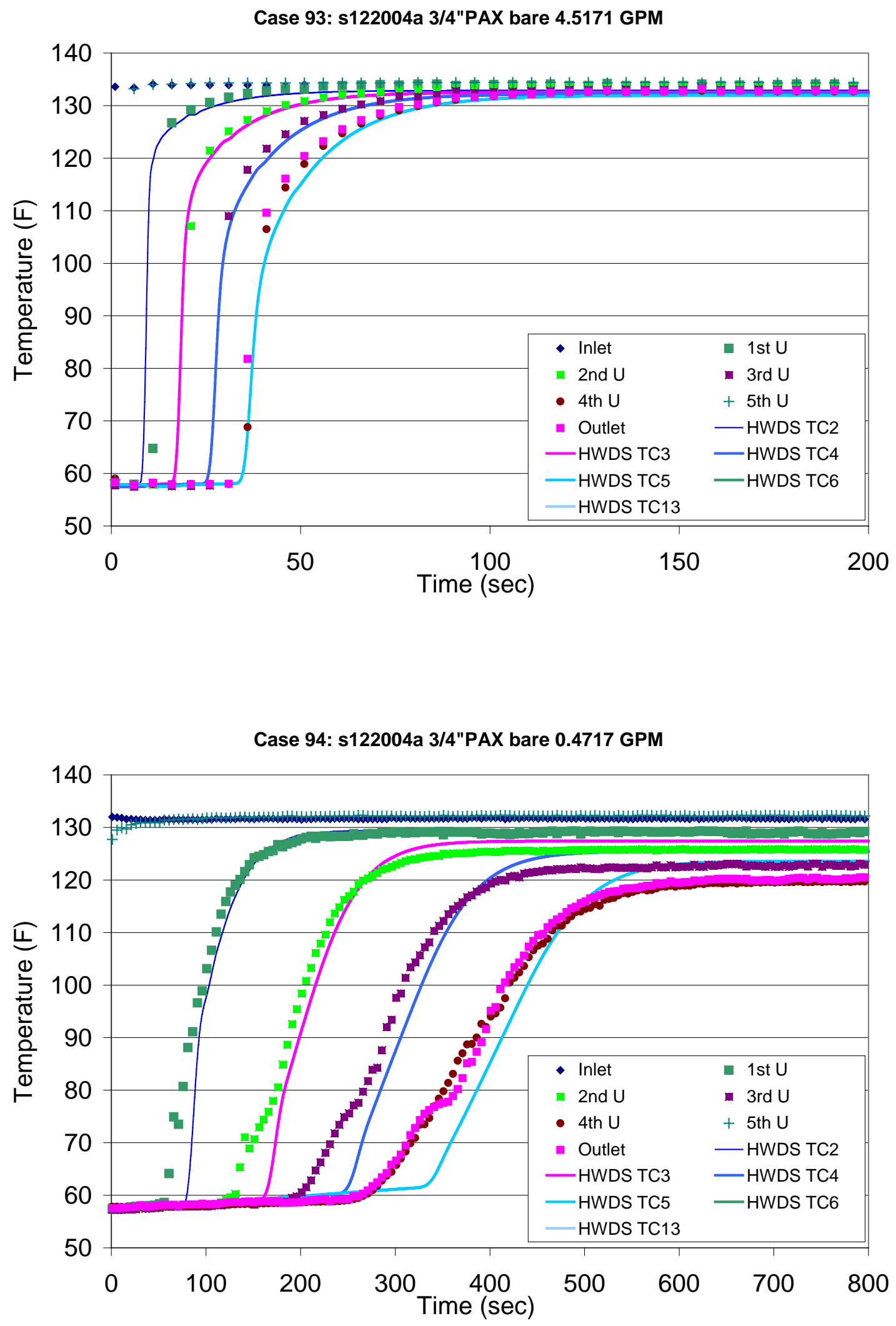
Case 95: s122004a 3/4"PAX bare 3.2384 GPM

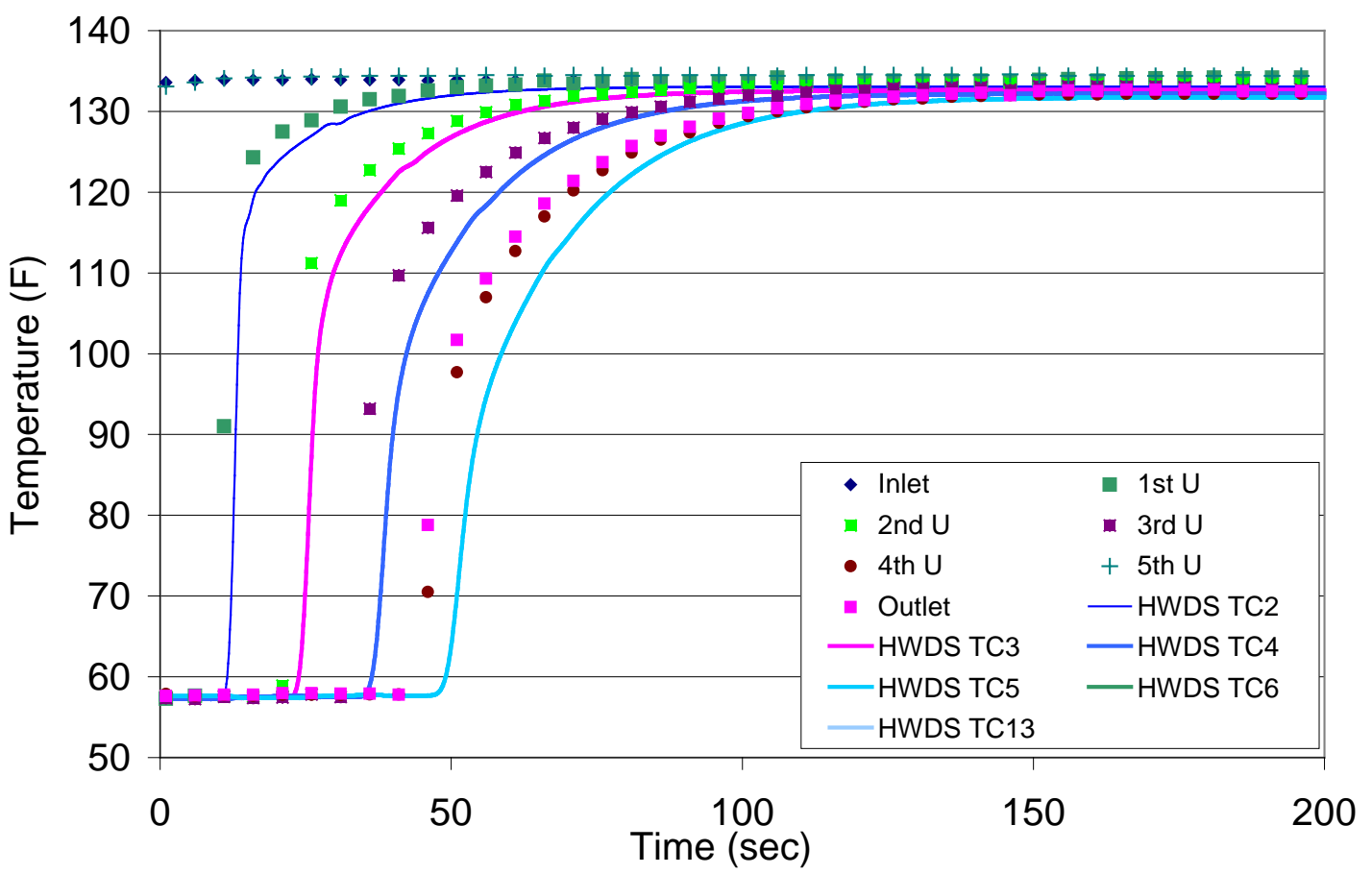

Case 96: s122004a 3/4"PAX bare 0.9362 GPM

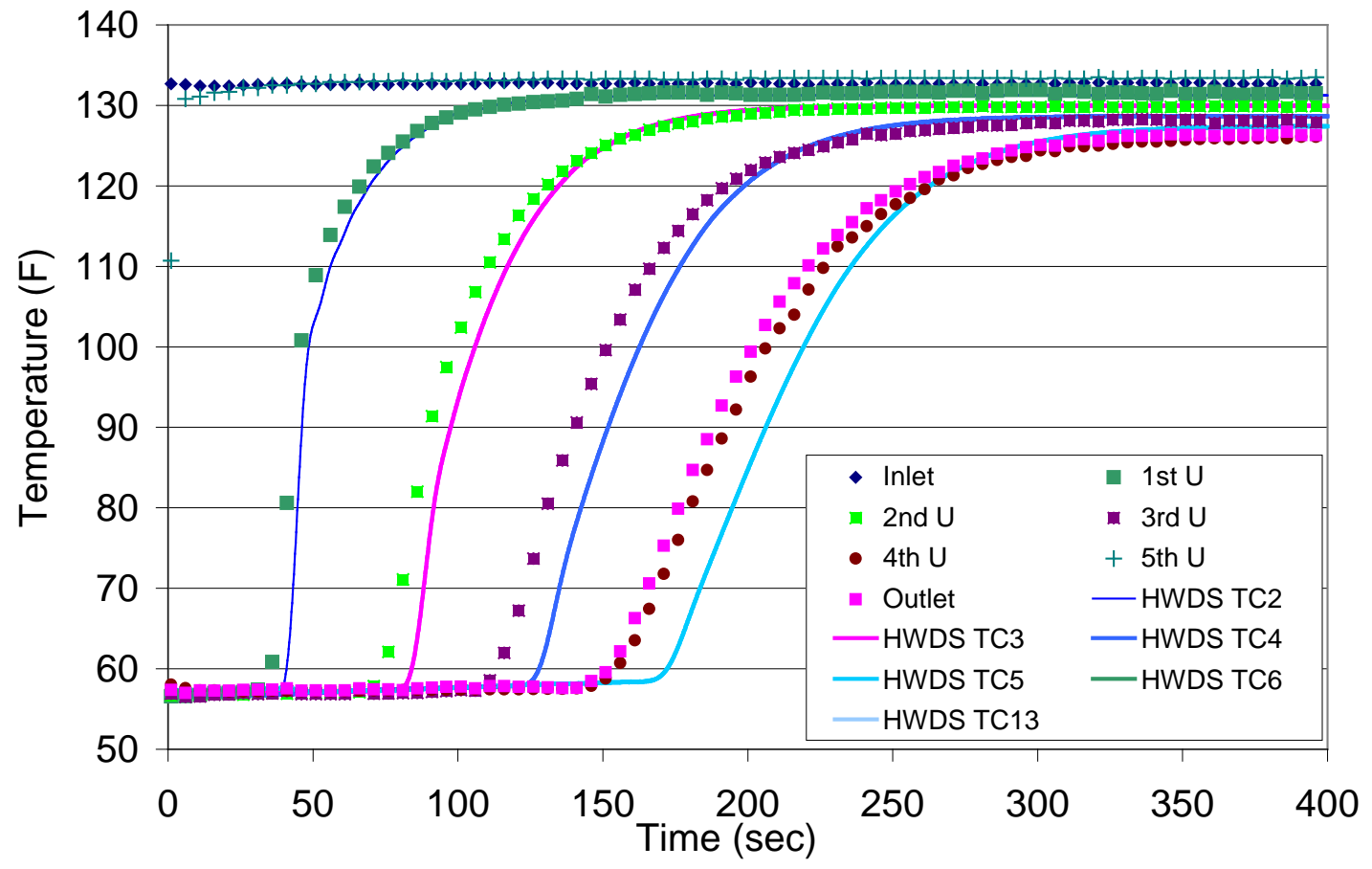



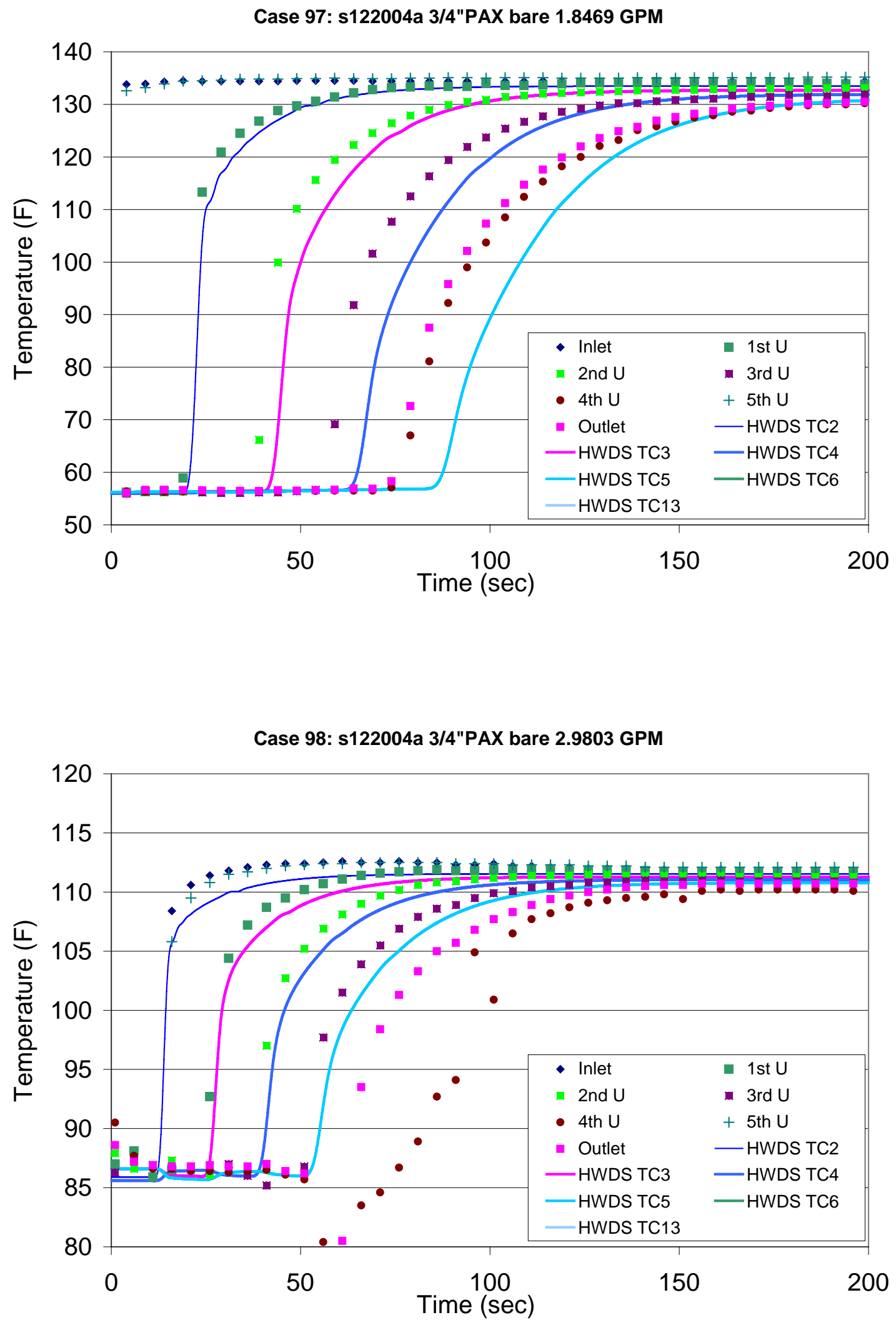

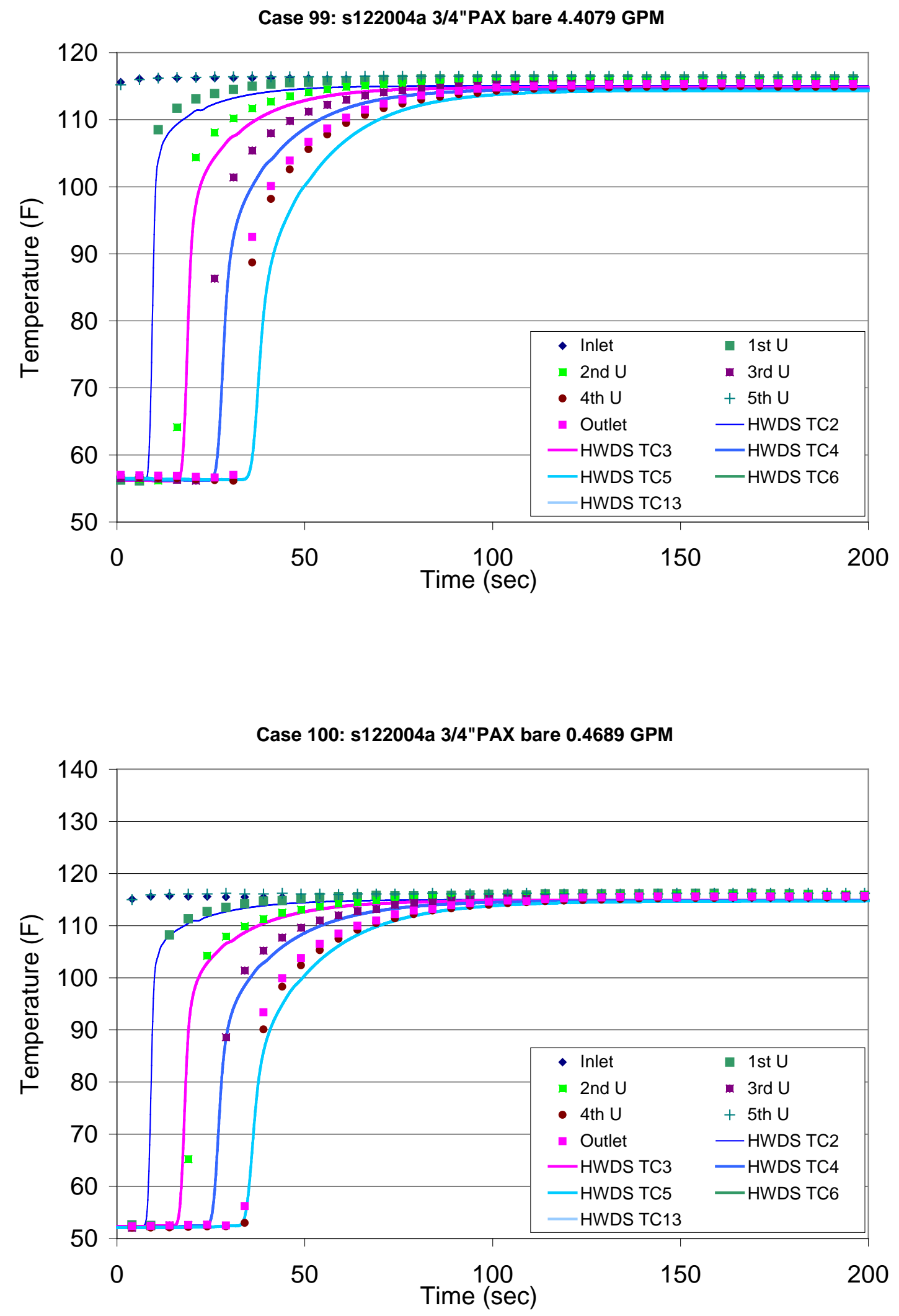


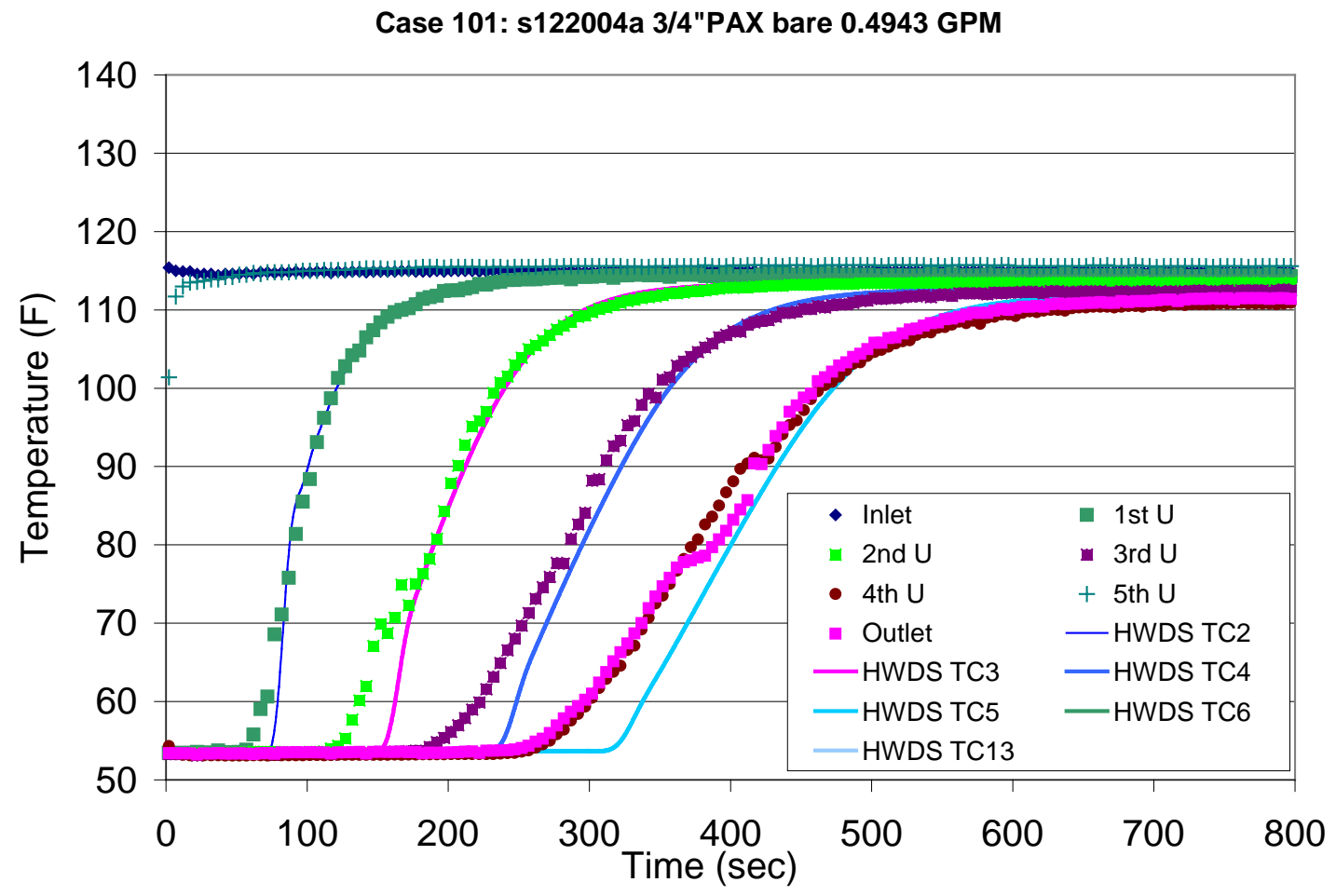

Case 102: s122004a 3/4"PAX bare 3.2001 GPM

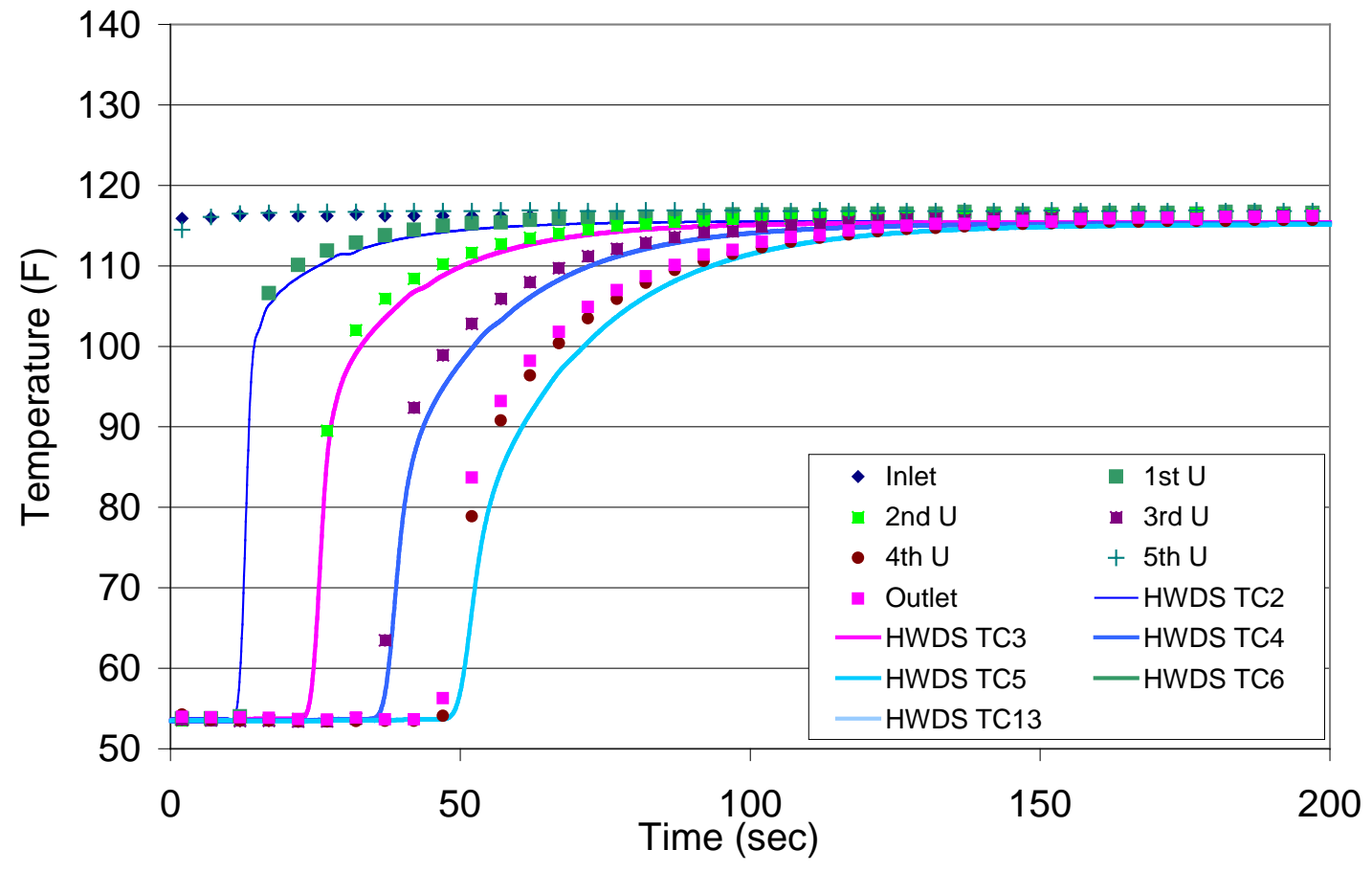




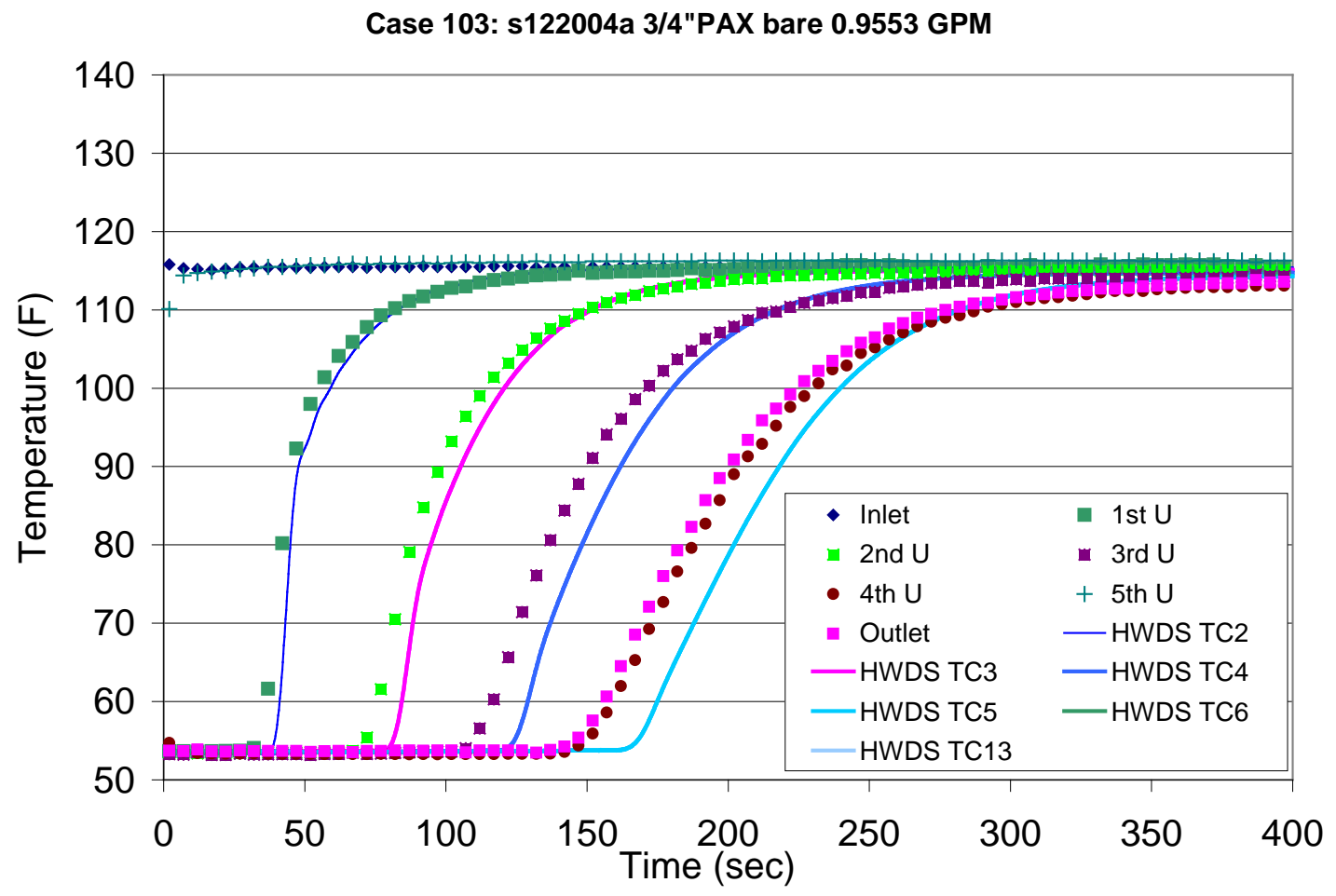

Case 104: s122004a 3/4"PAX bare 1.9013 GPM

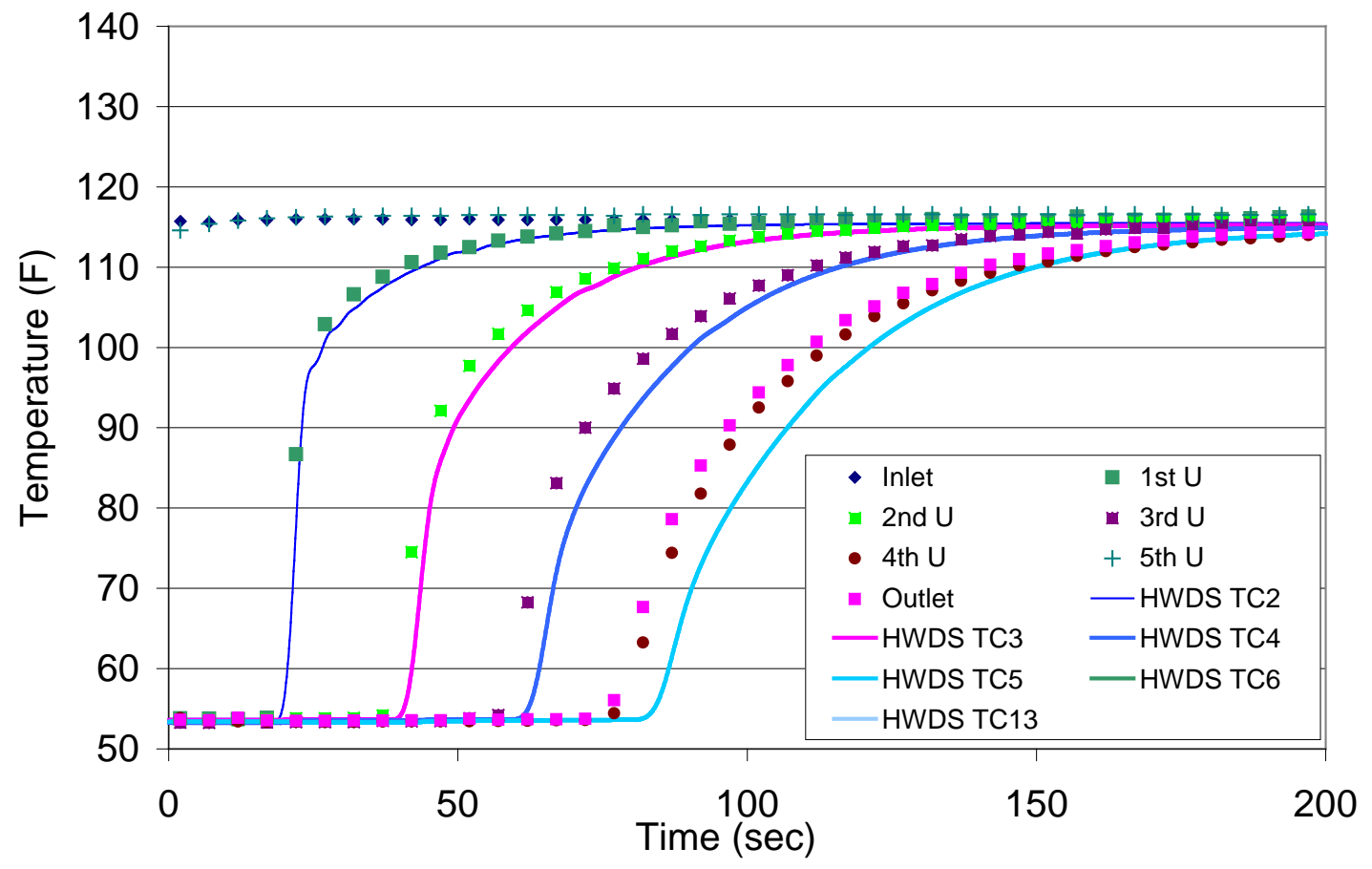



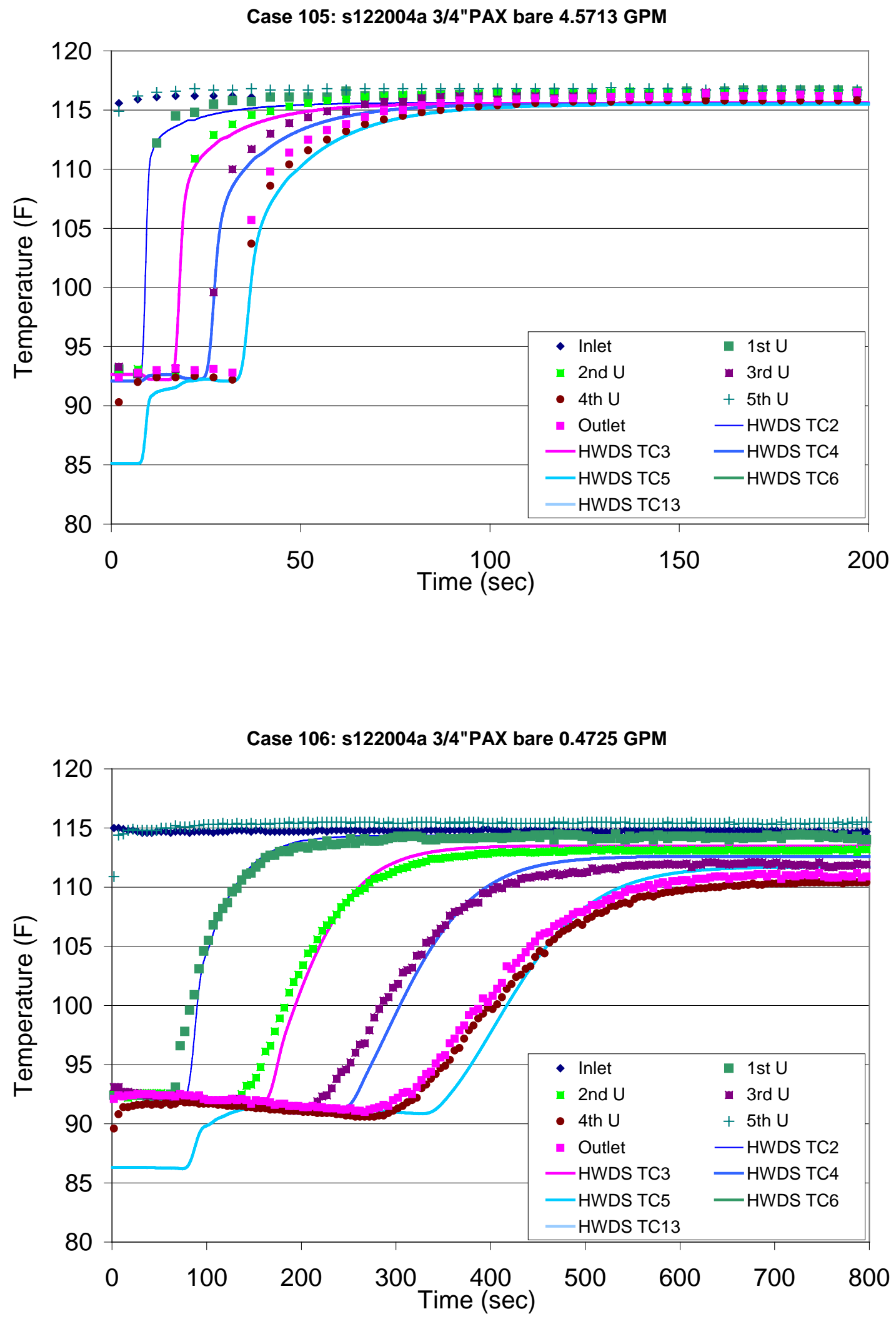

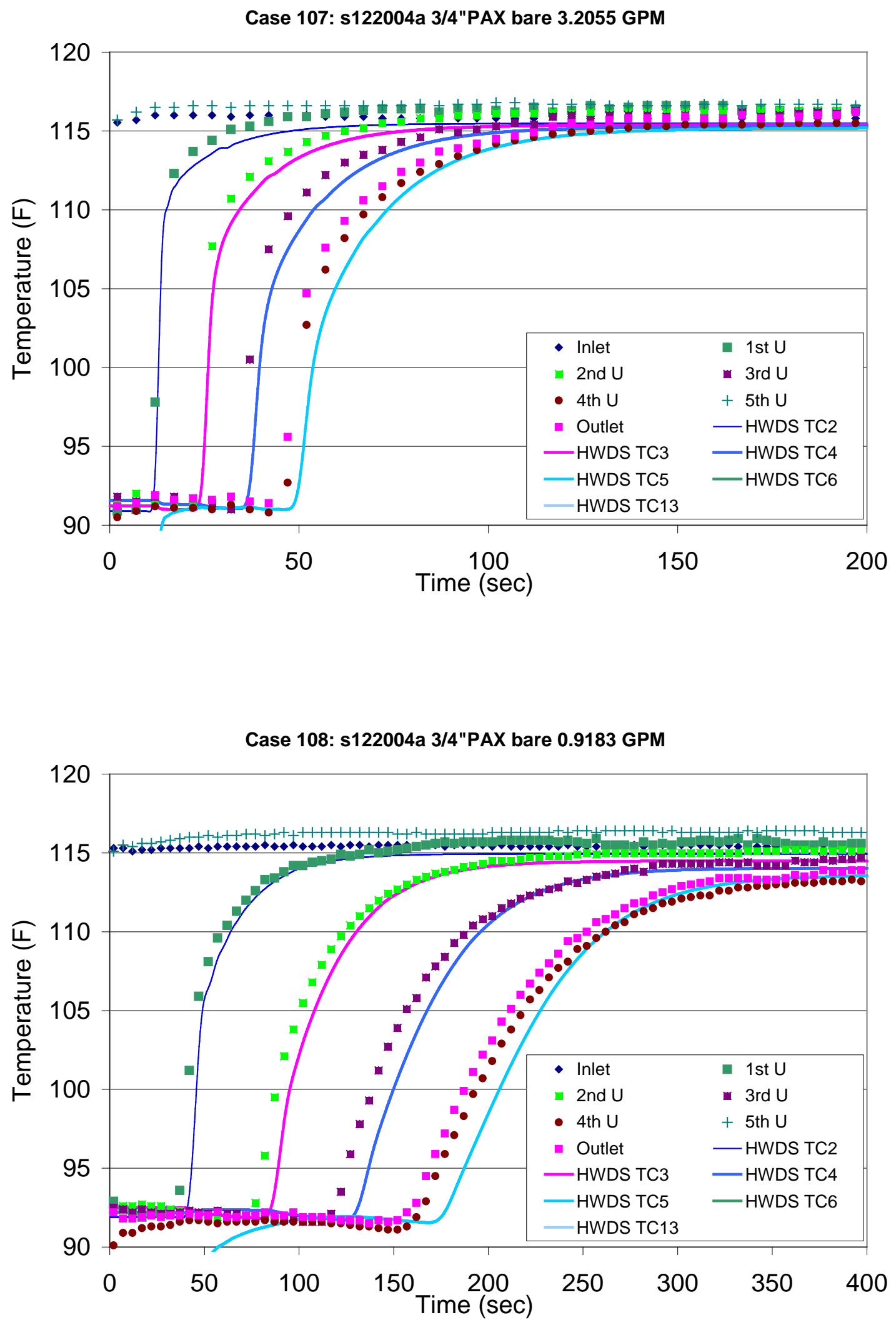

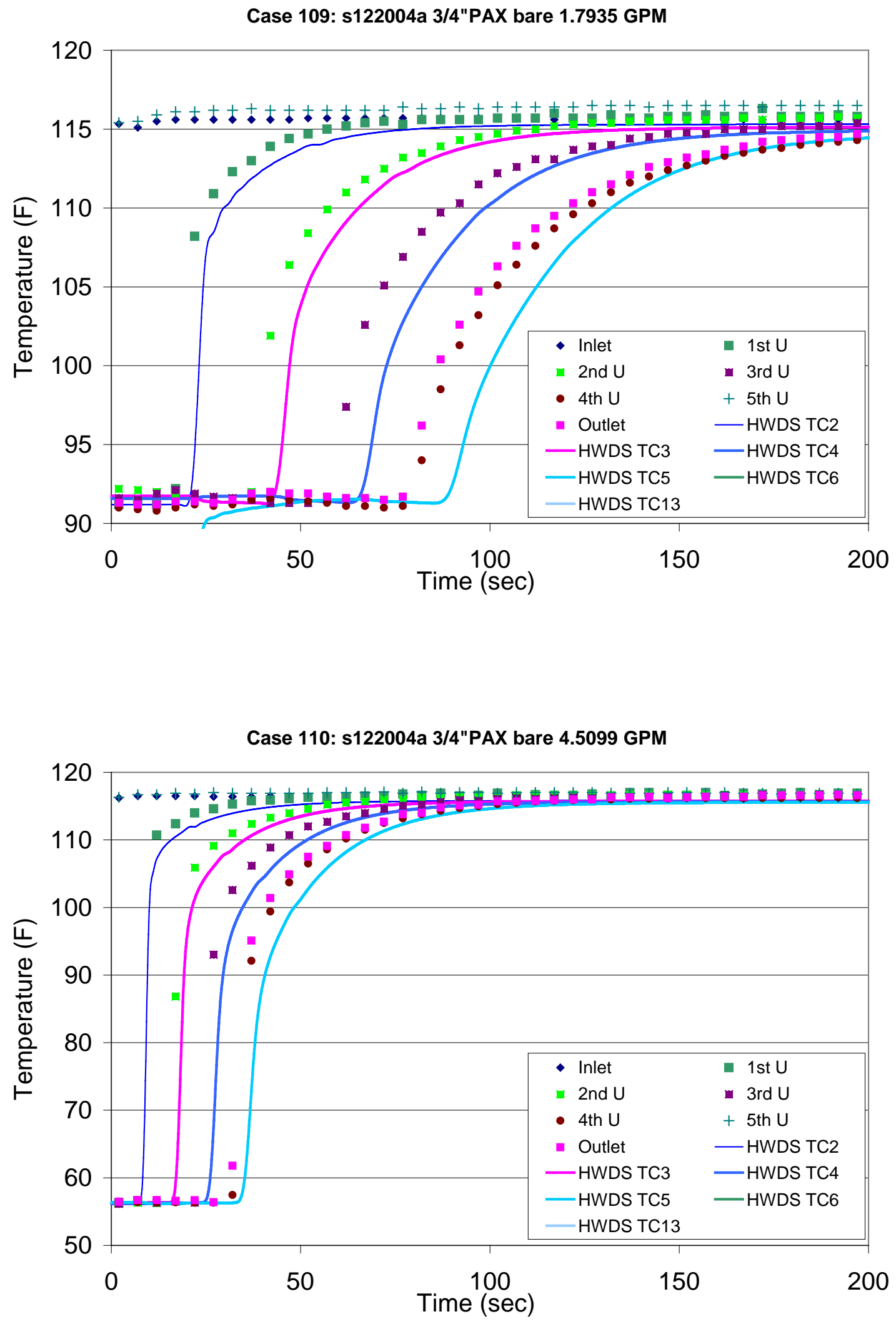

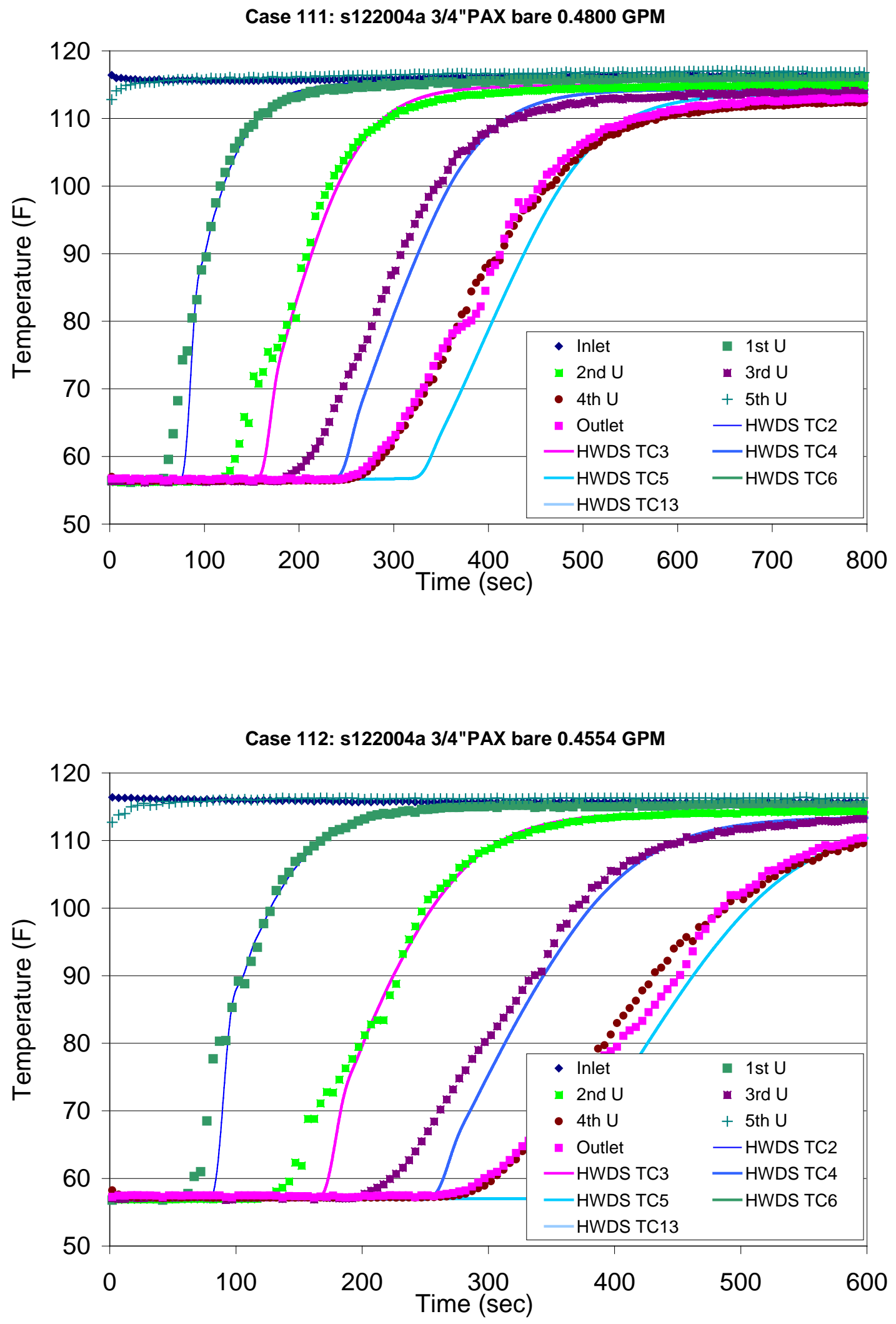


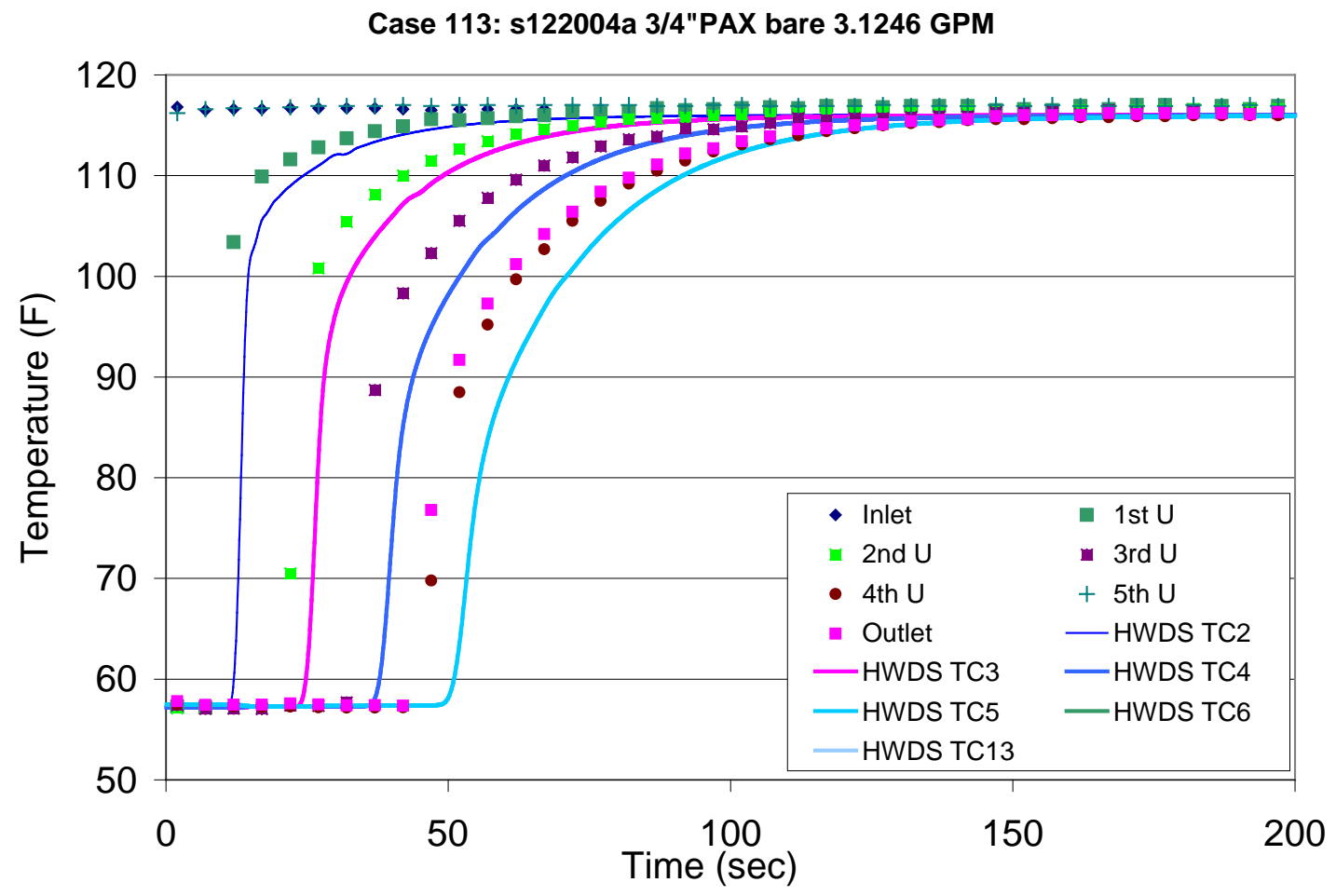

Case 114: s122004a 3/4"PAX bare 4.4565 GPM

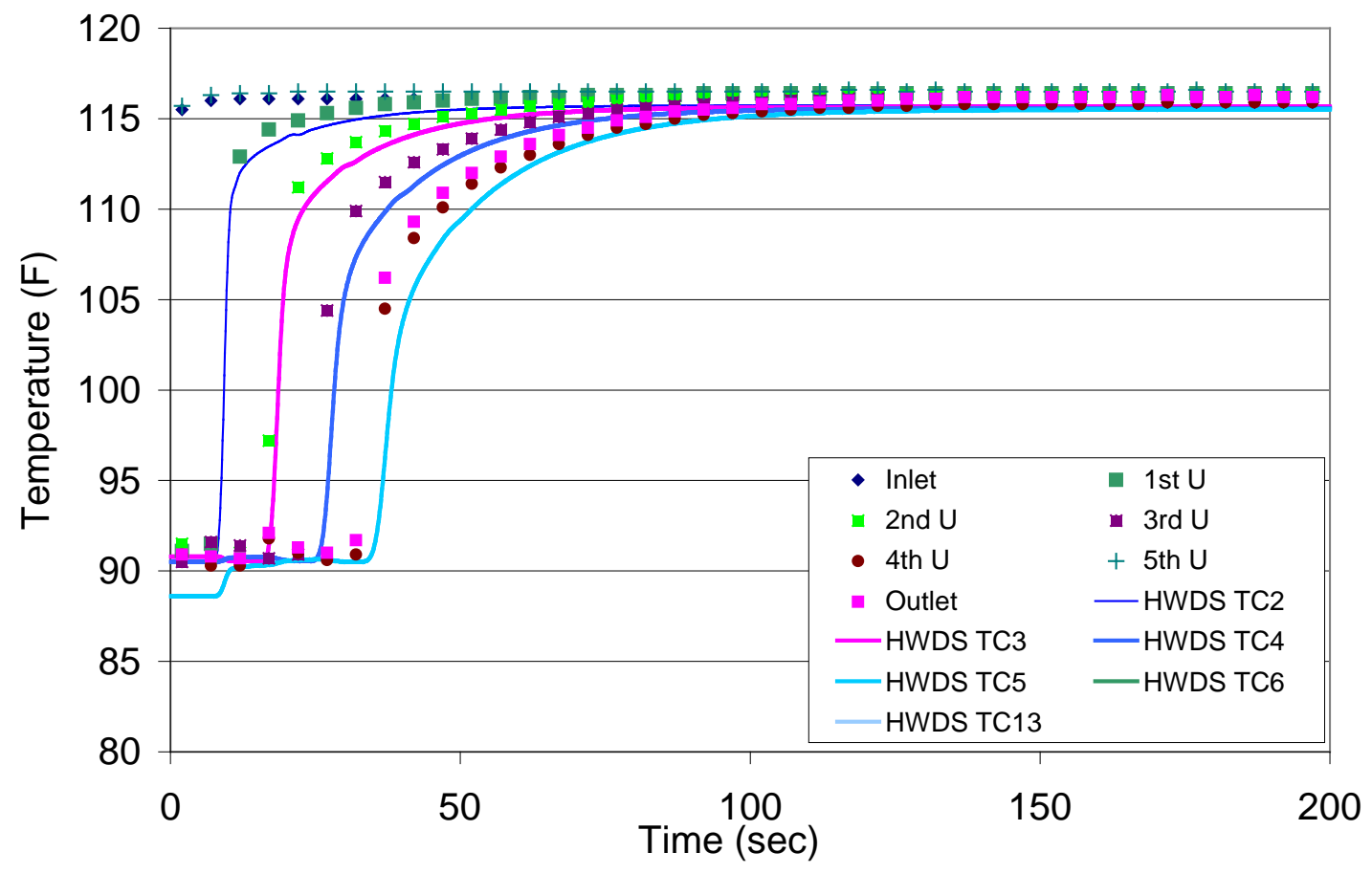



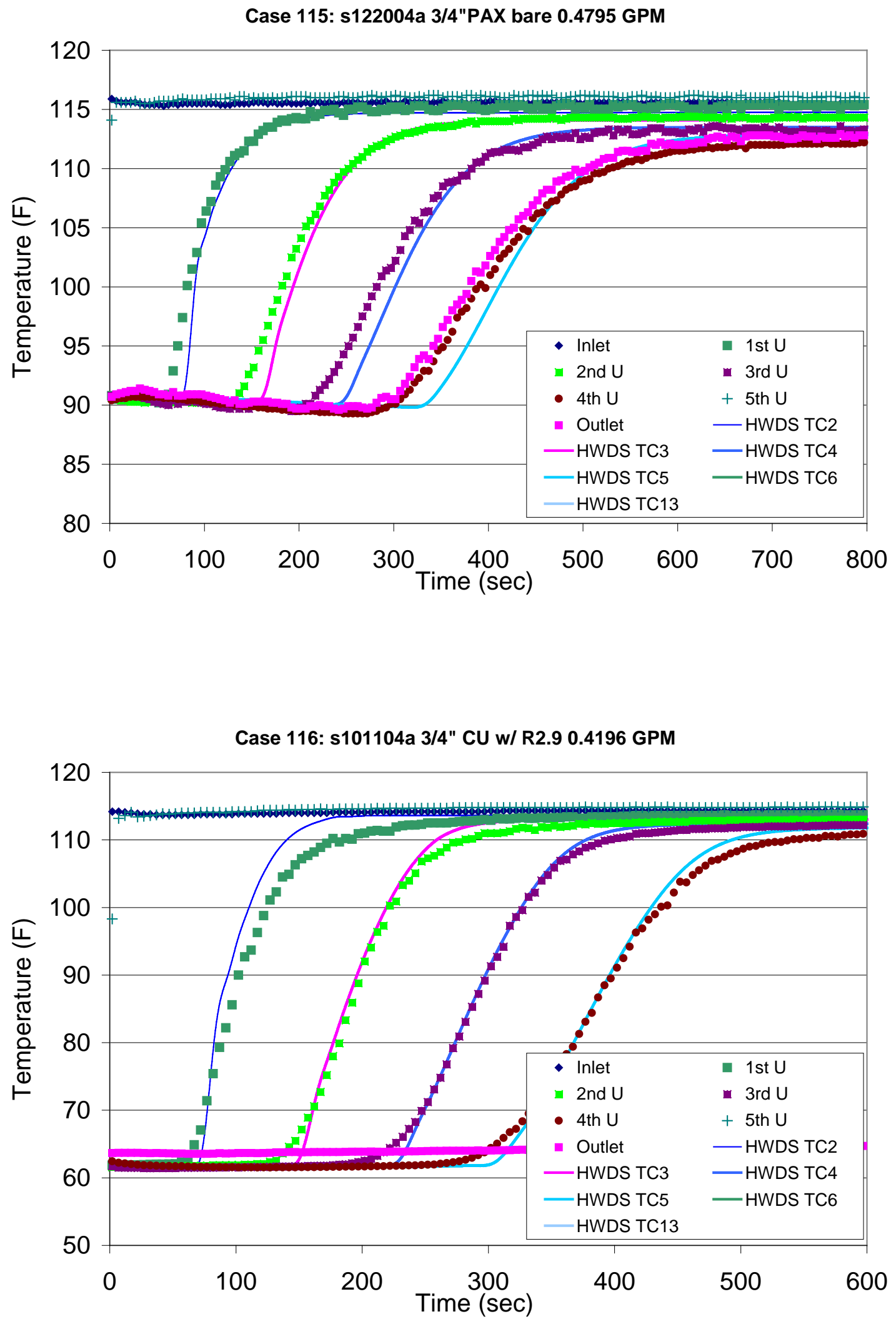


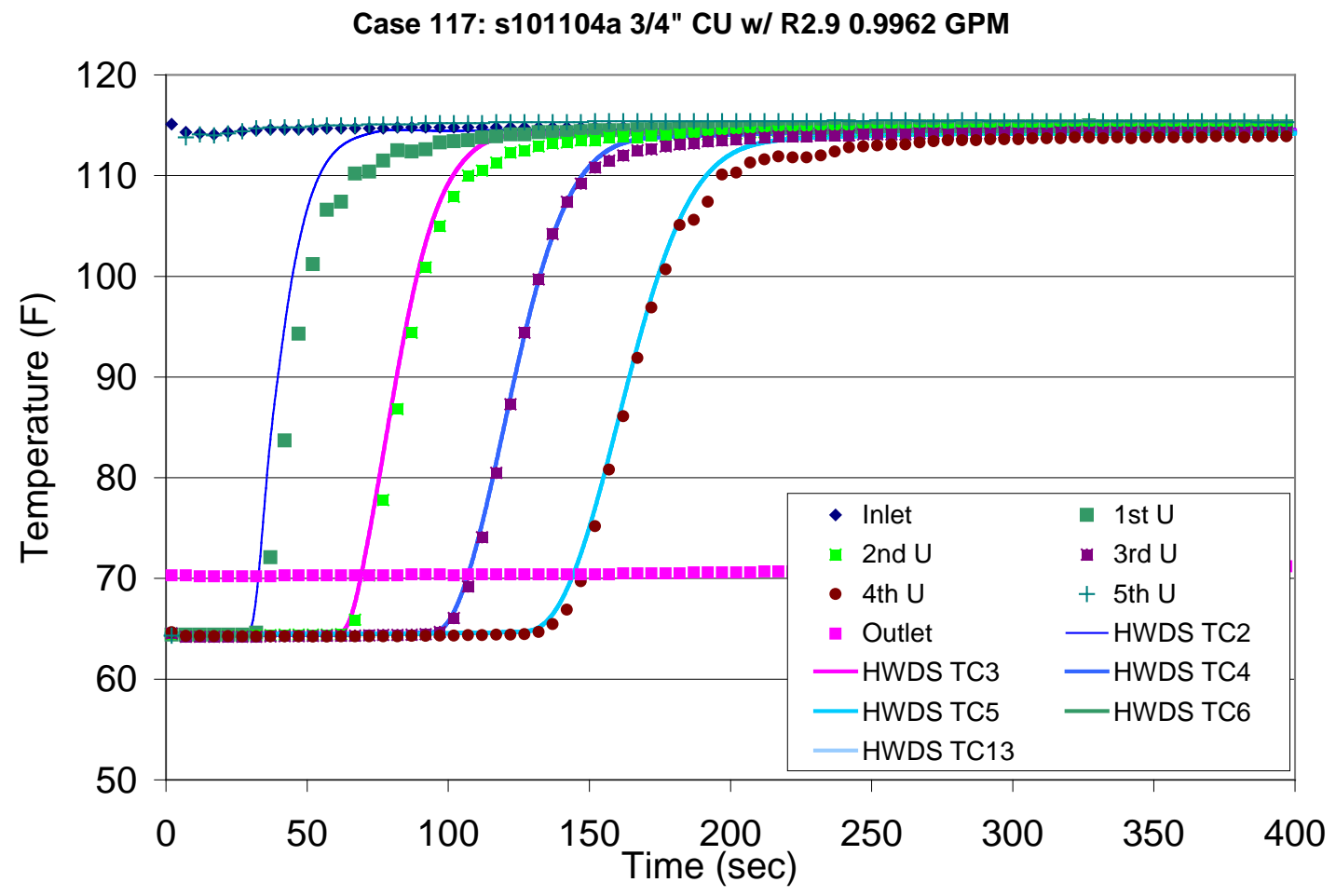

Case 118: s101104a 3/4" CU w/ R2.9 2.1416 GPM

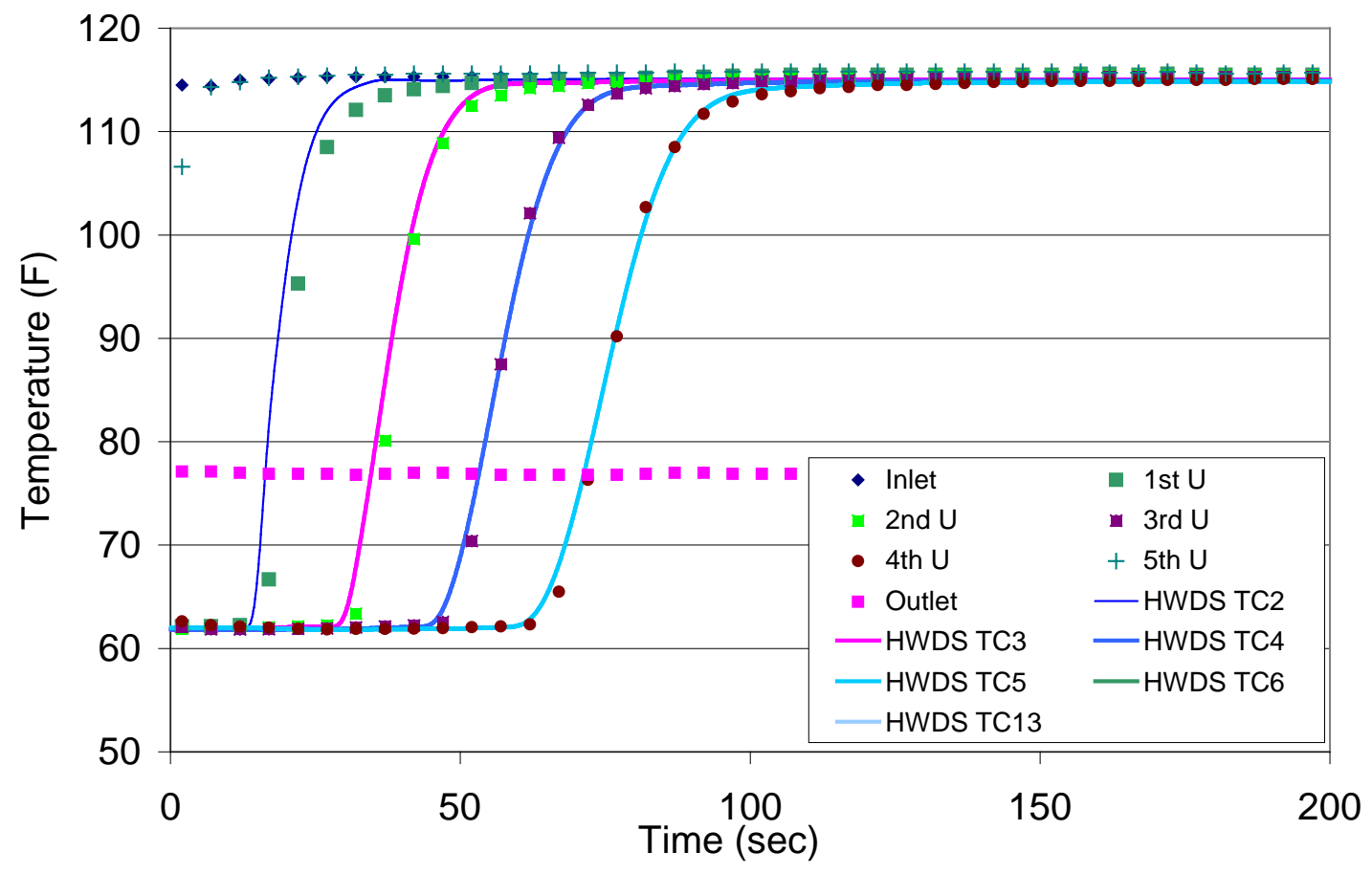




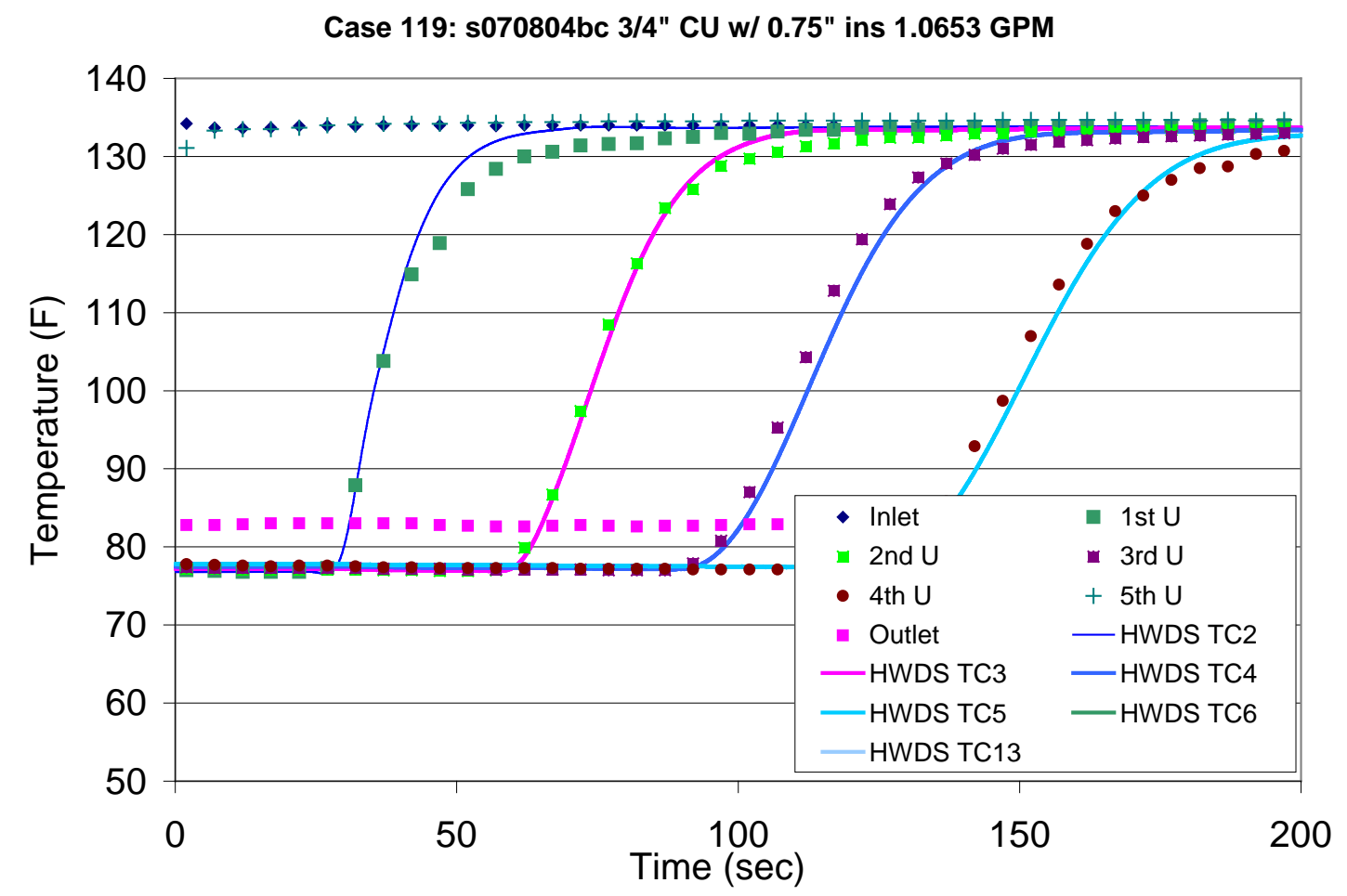

Case 120: s100604 3/4" CU BARE 1.8107 GPM

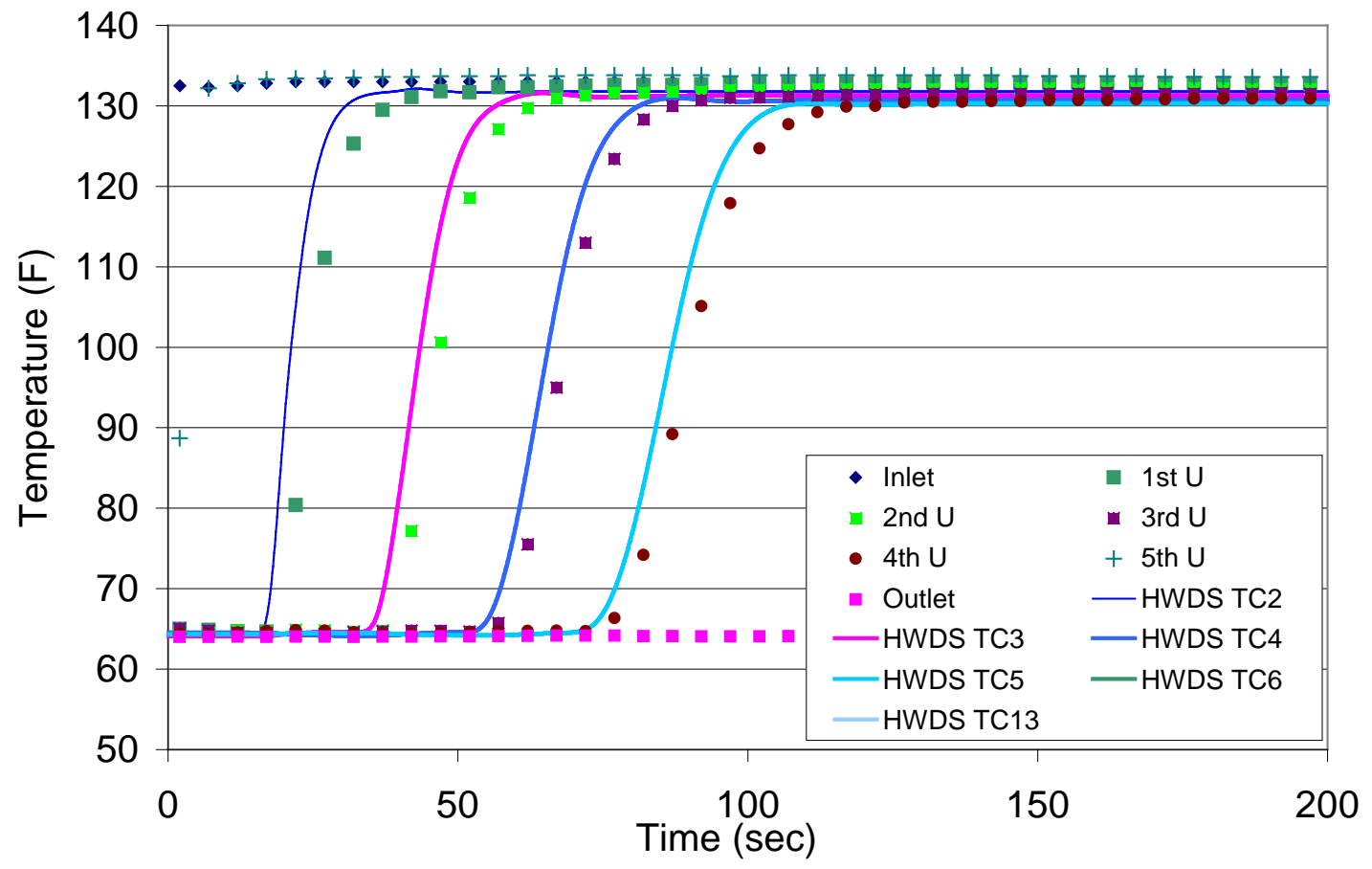



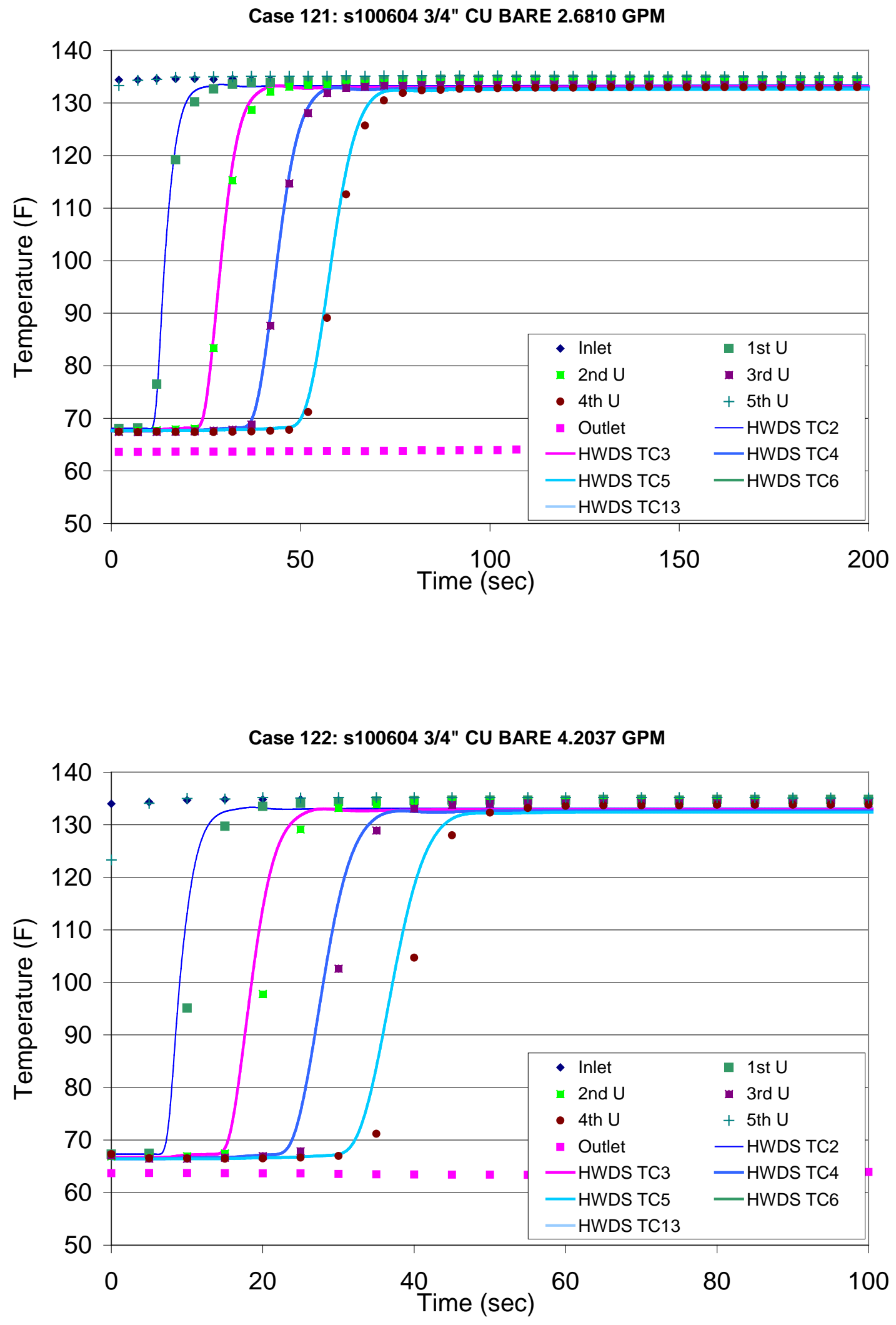

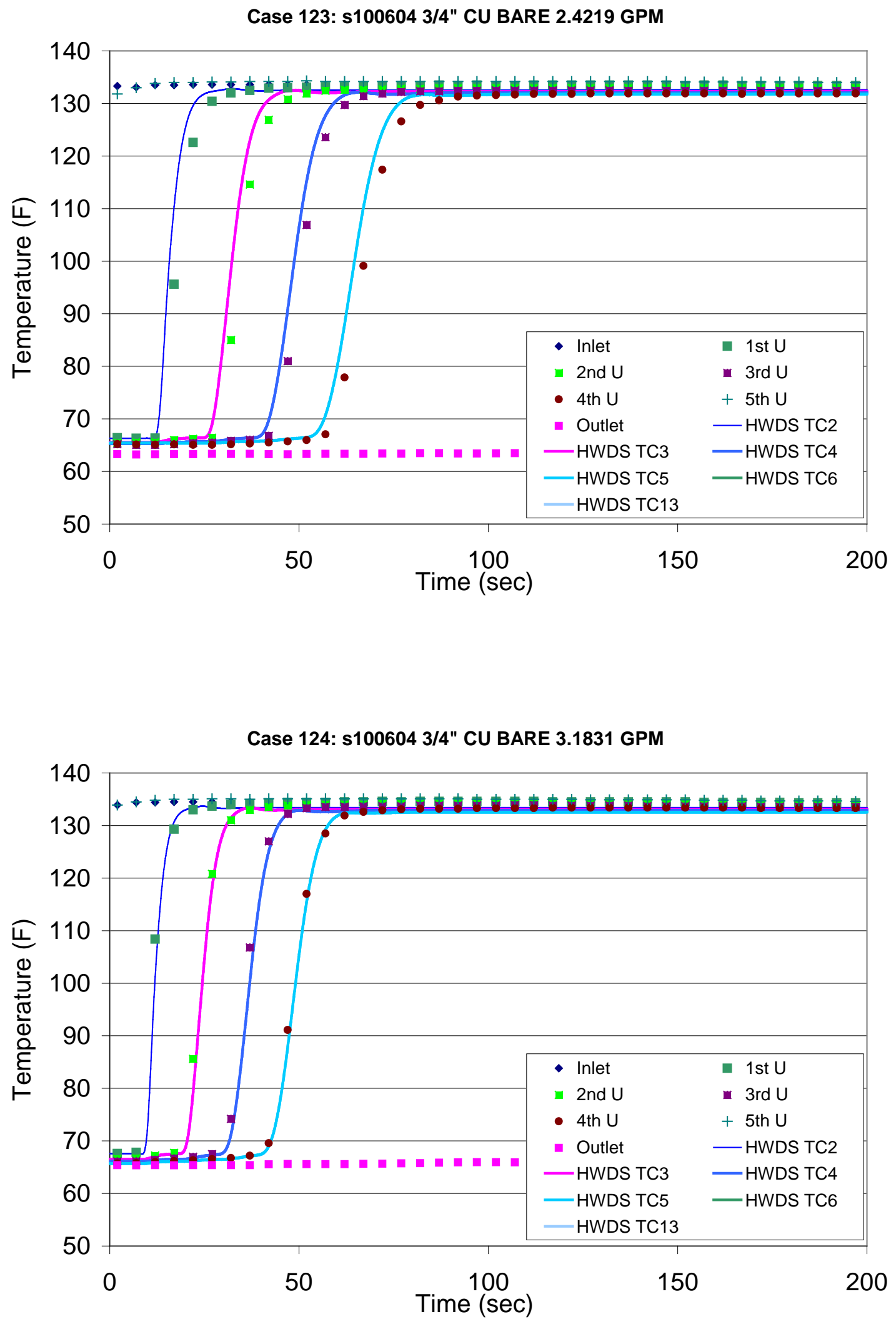
Case 125: s100604 3/4" CU BARE 1.6735 GPM

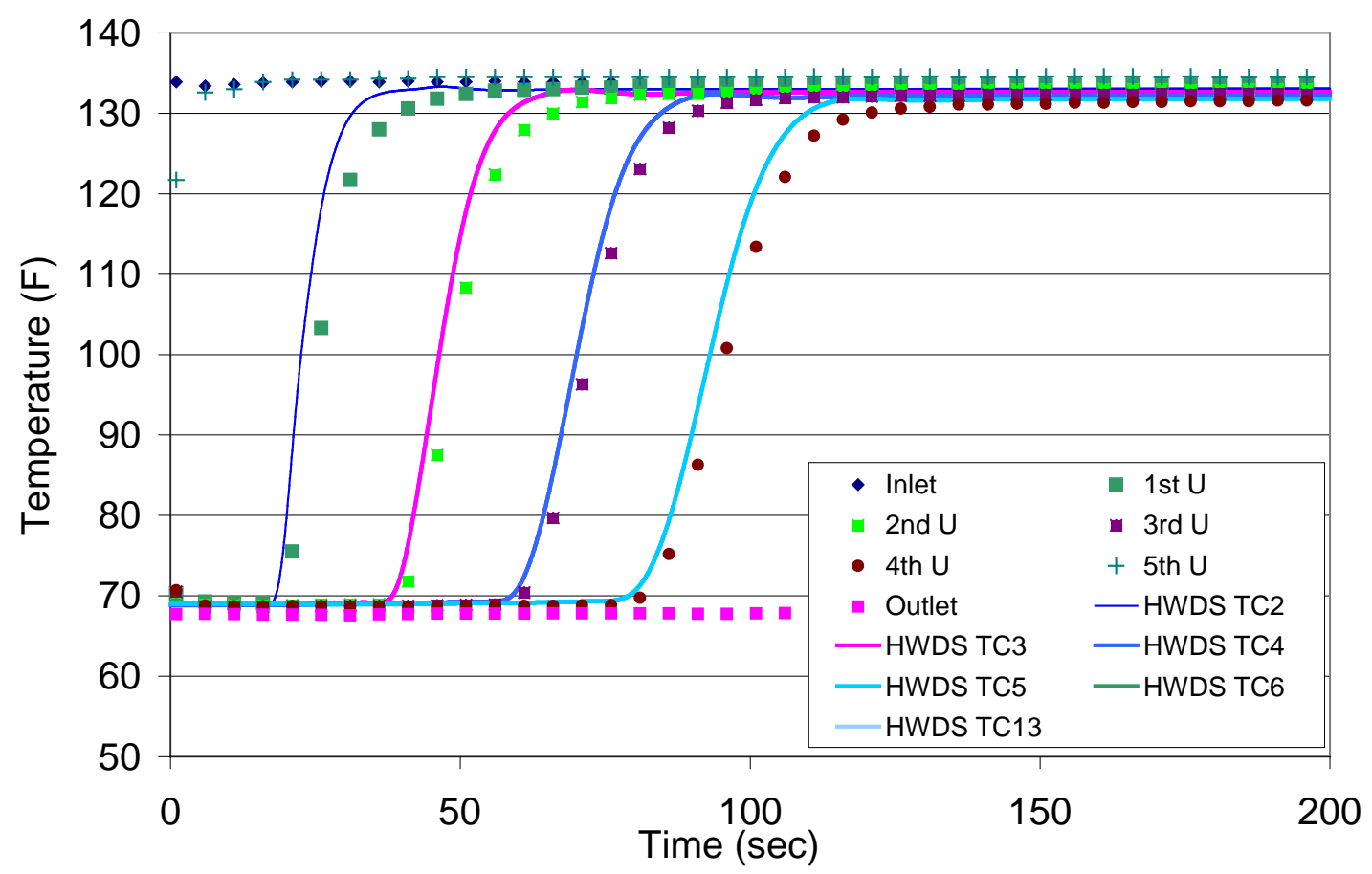

Case 126: s100604 3/4" CU BARE 0.8747 GPM

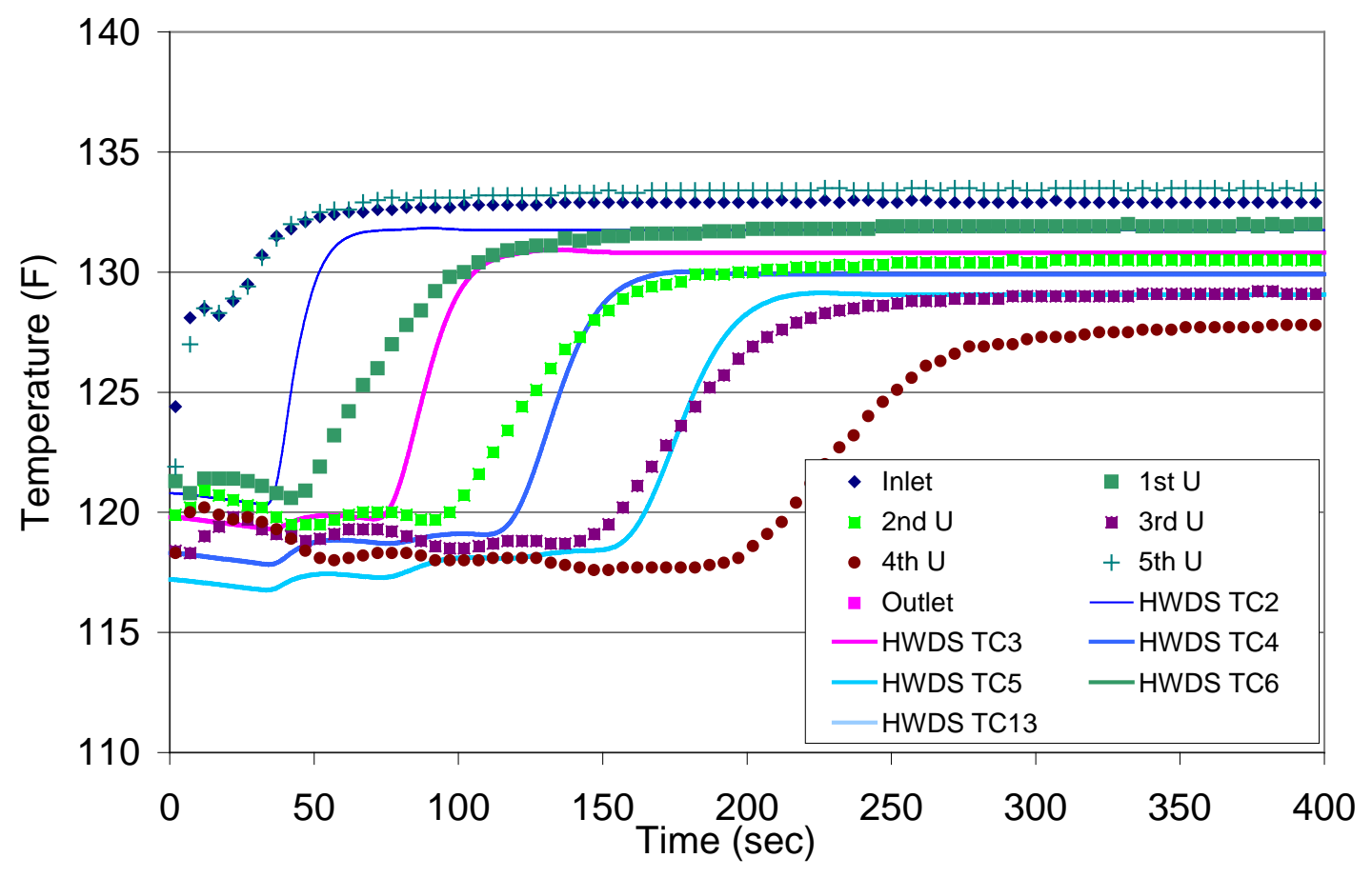



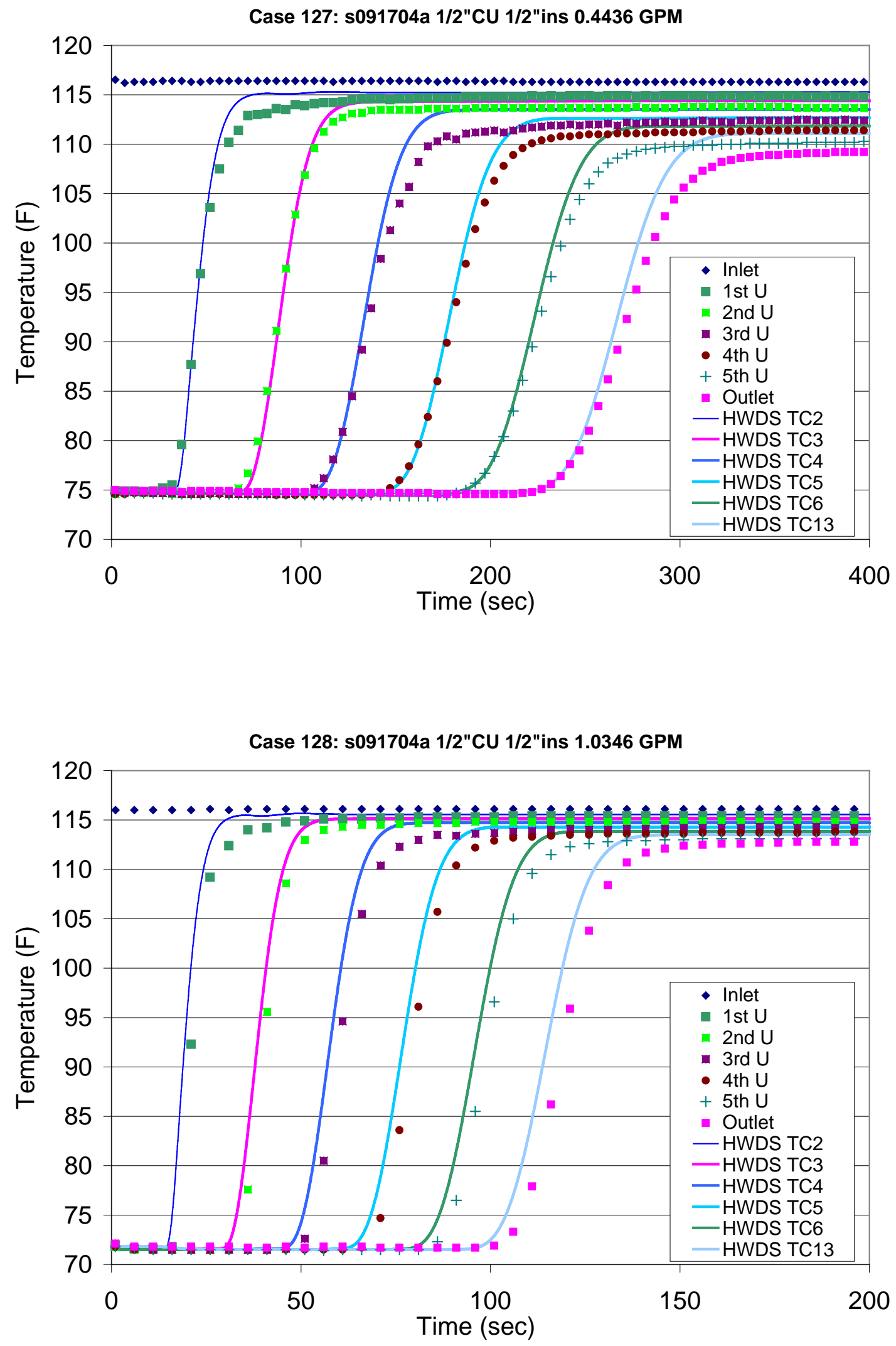

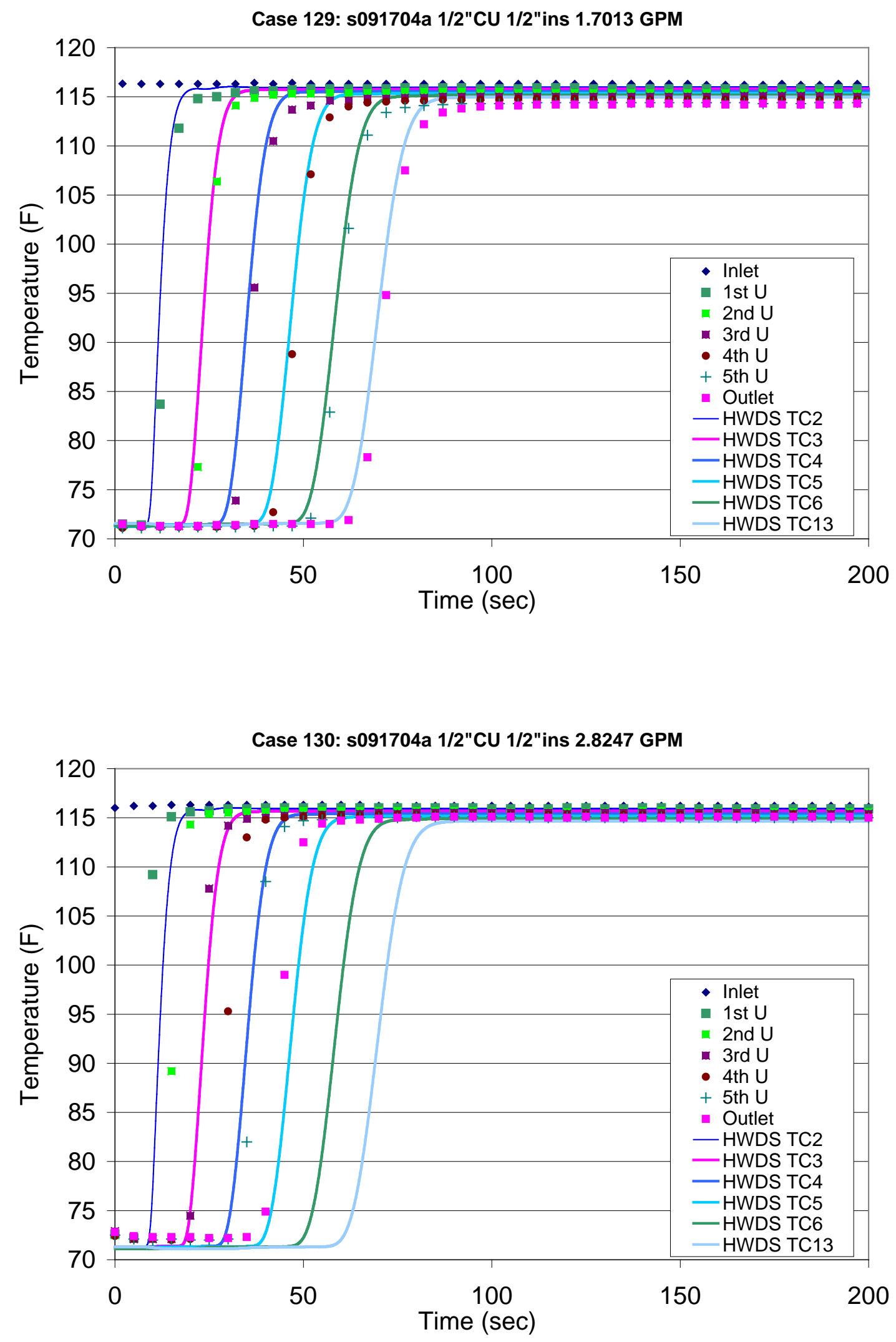

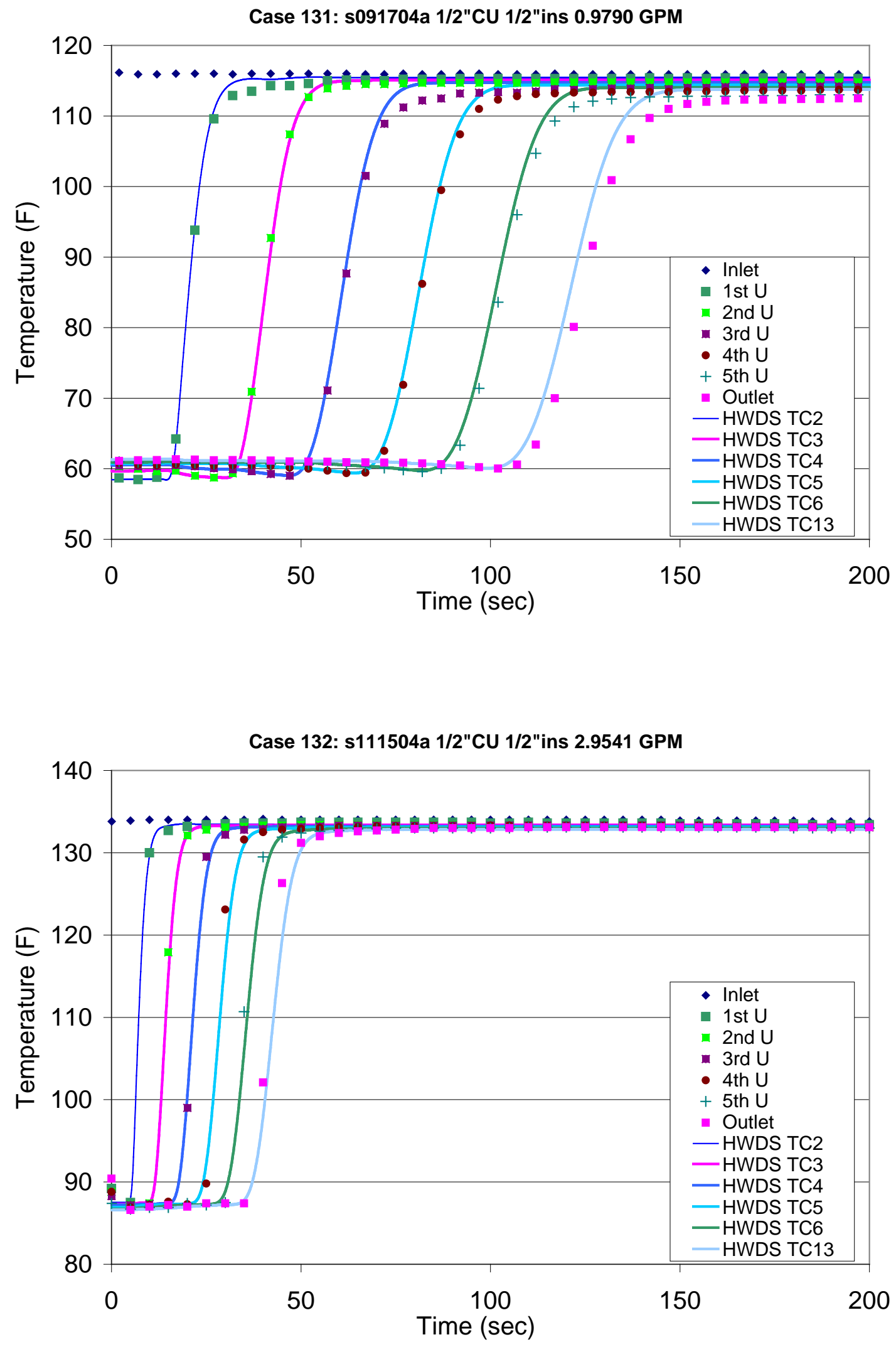


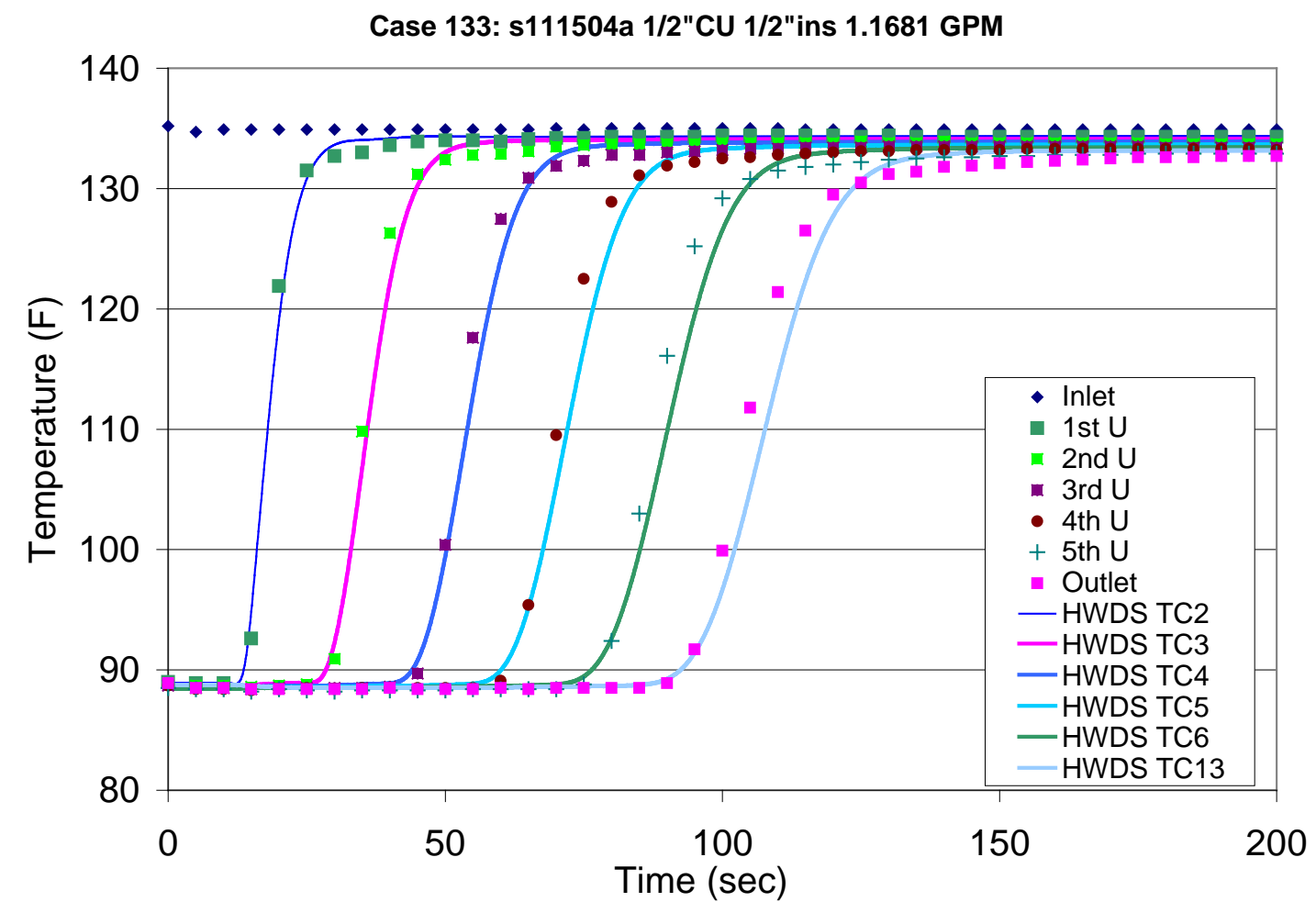


Appendix C. TABULAR RESULTS FROM HWDS FOR BURIED PIPES ${ }^{\star}$

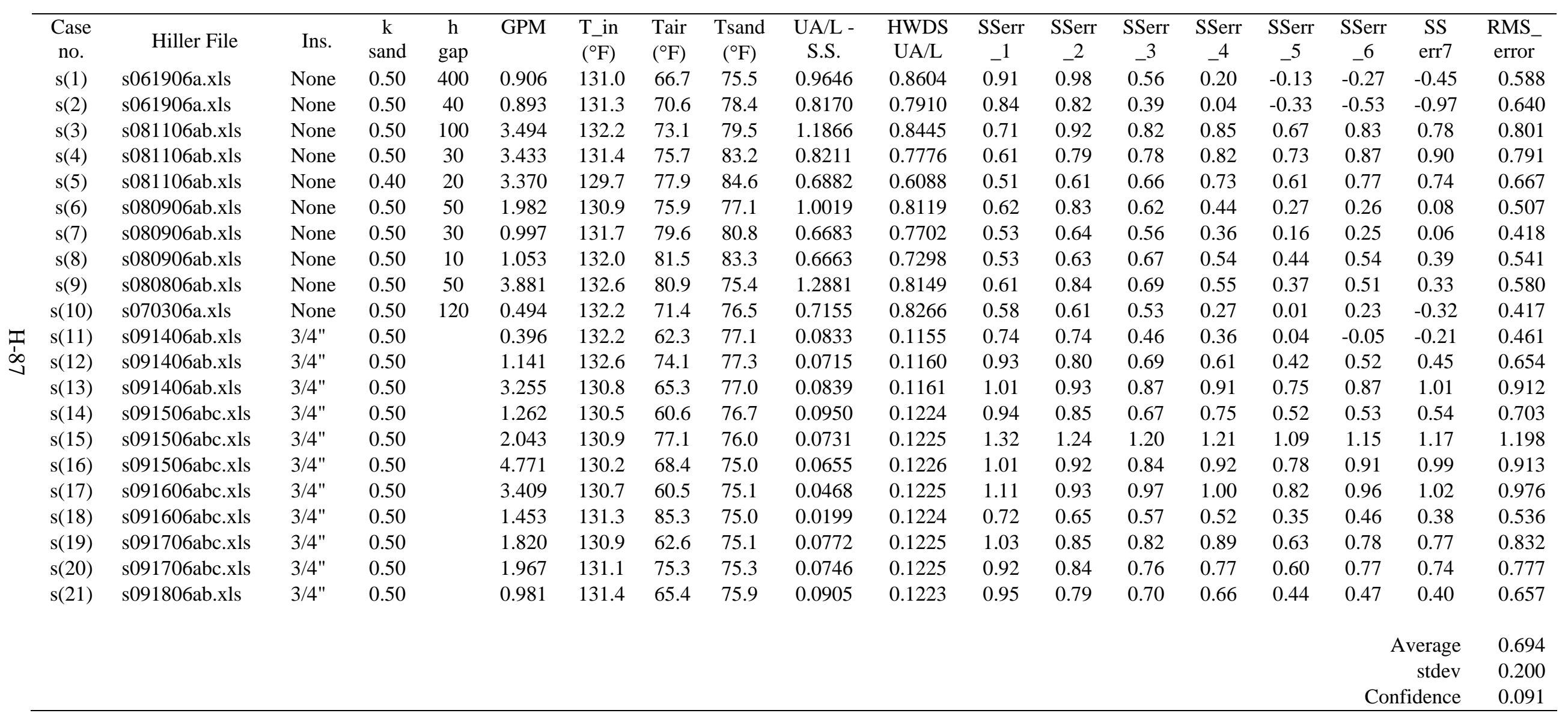

\footnotetext{
"All pipe is $3 / 4$ " copper.
} 



\section{Appendix D. GRAPHICAL COMPARISON OF HWDS RESULTS TO MEASURED DATA FOR BURIED PIPE}
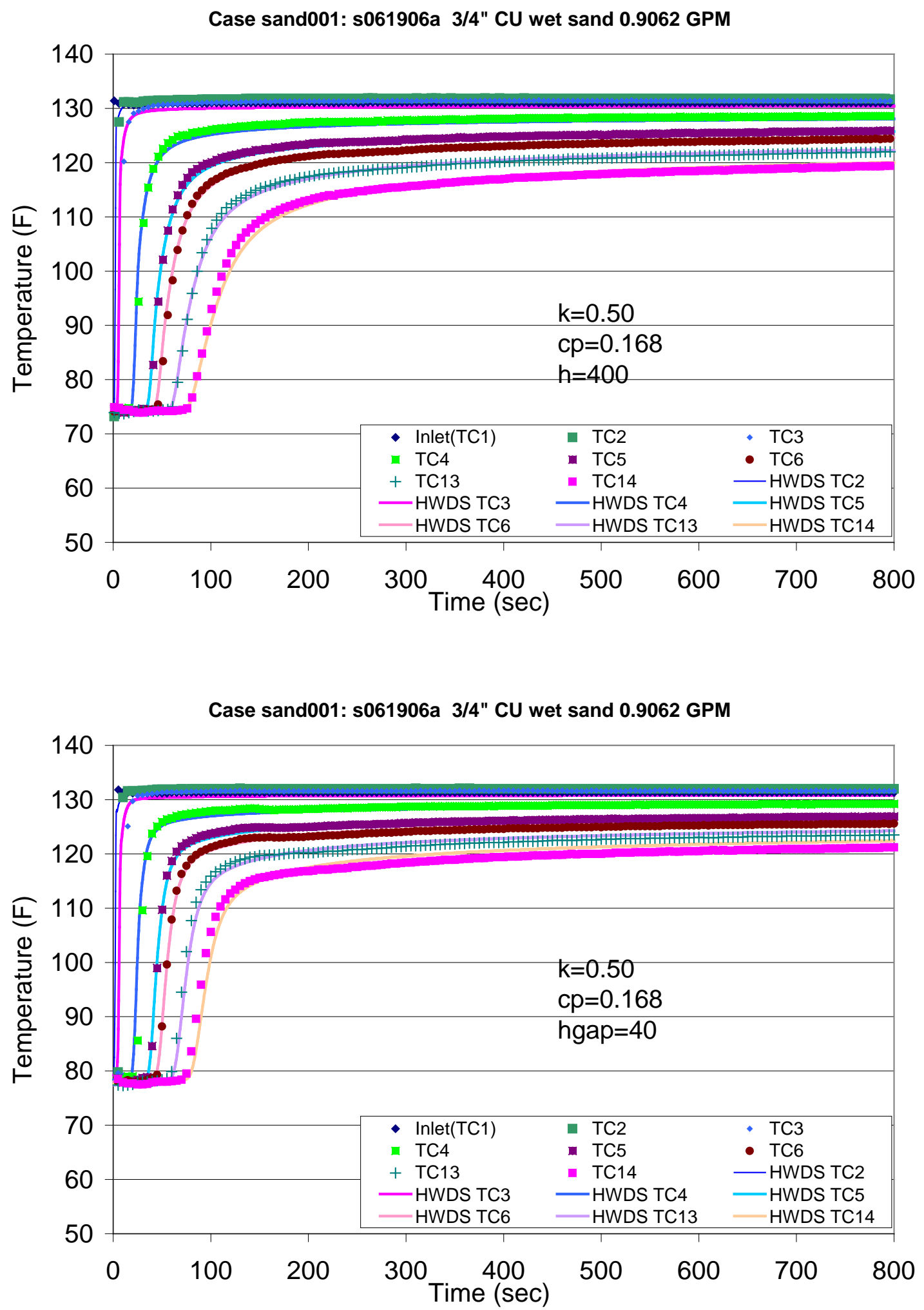

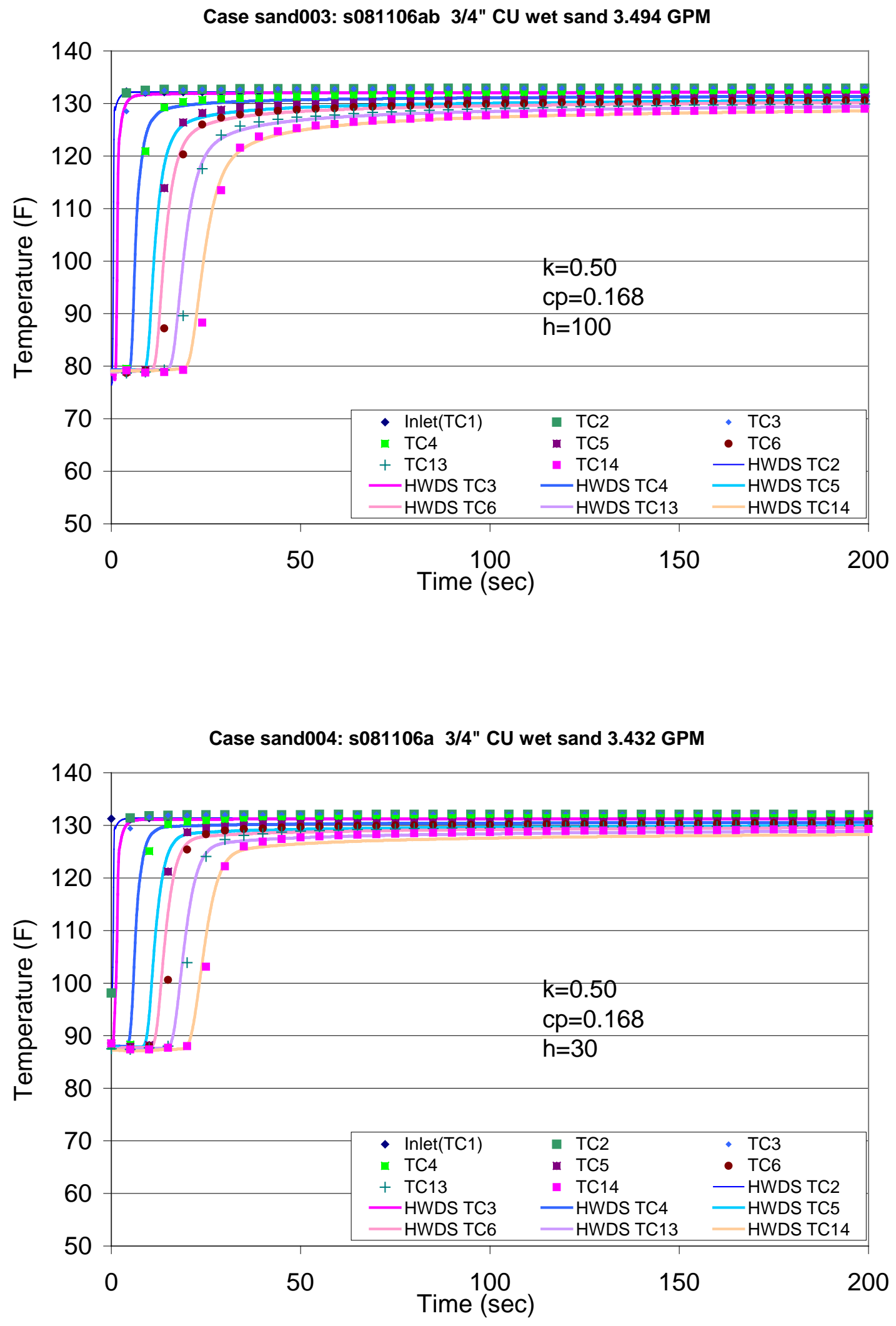

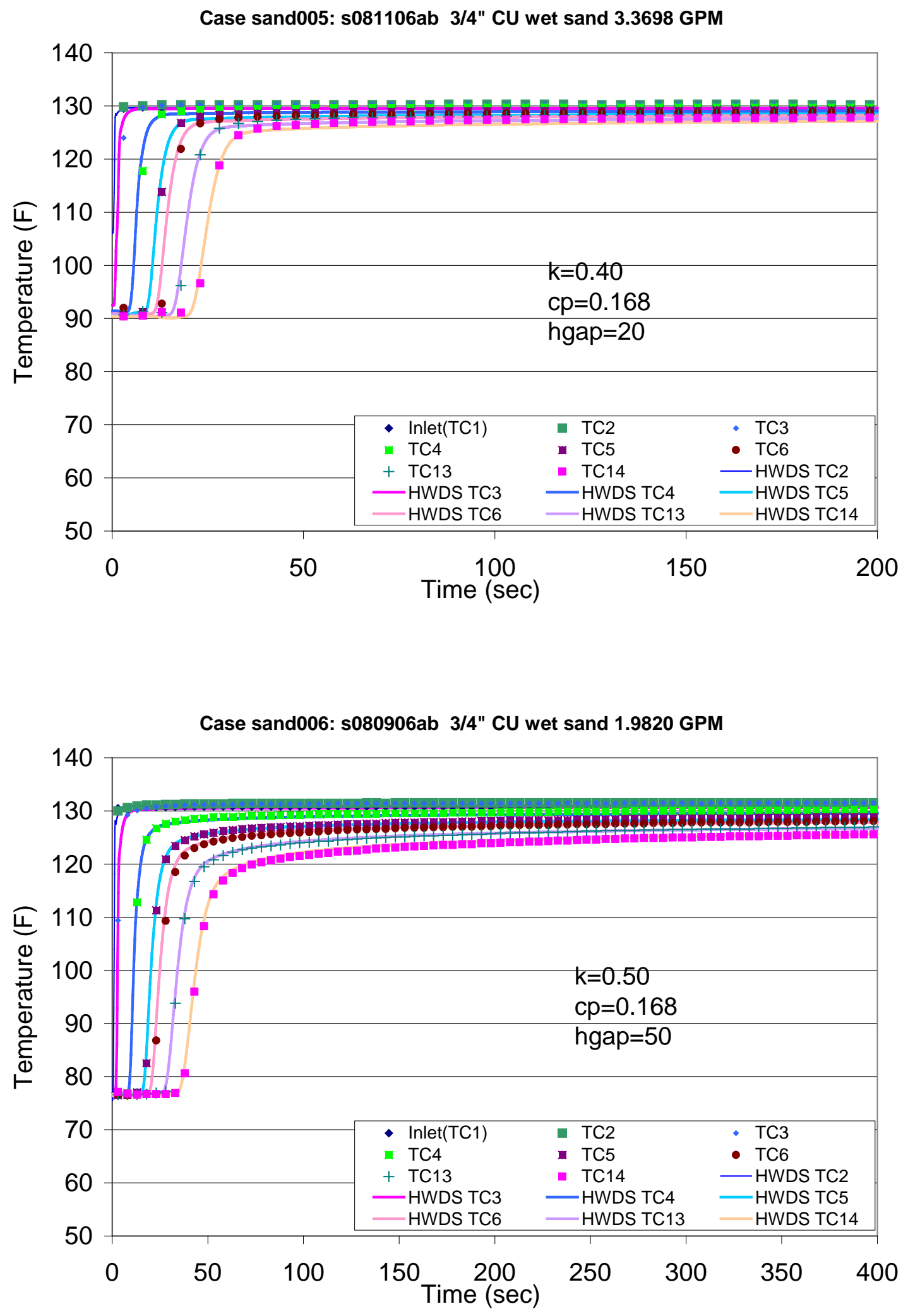


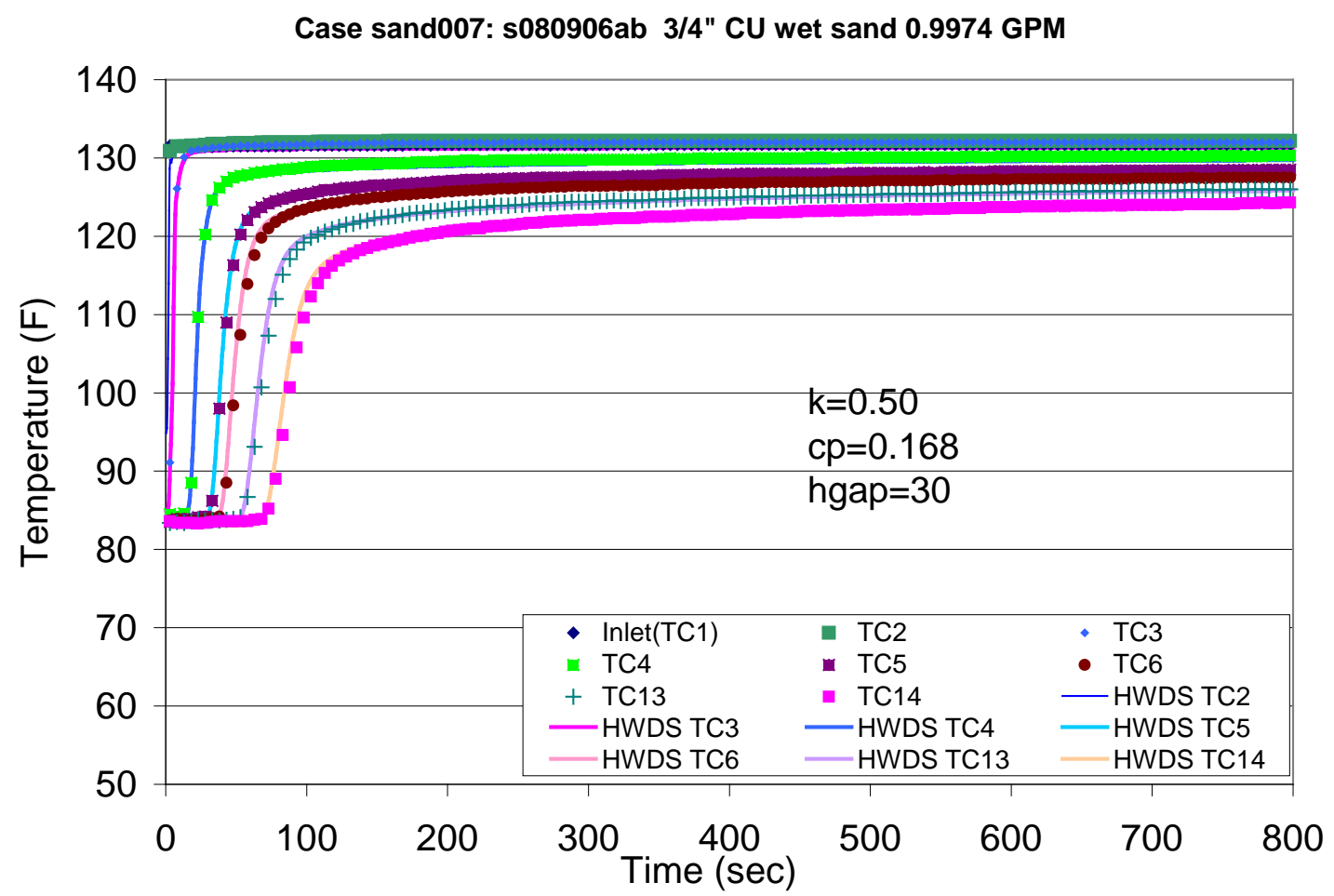

Case sand008: s080906ab 3/4" CU wet sand 1.0562 GPM

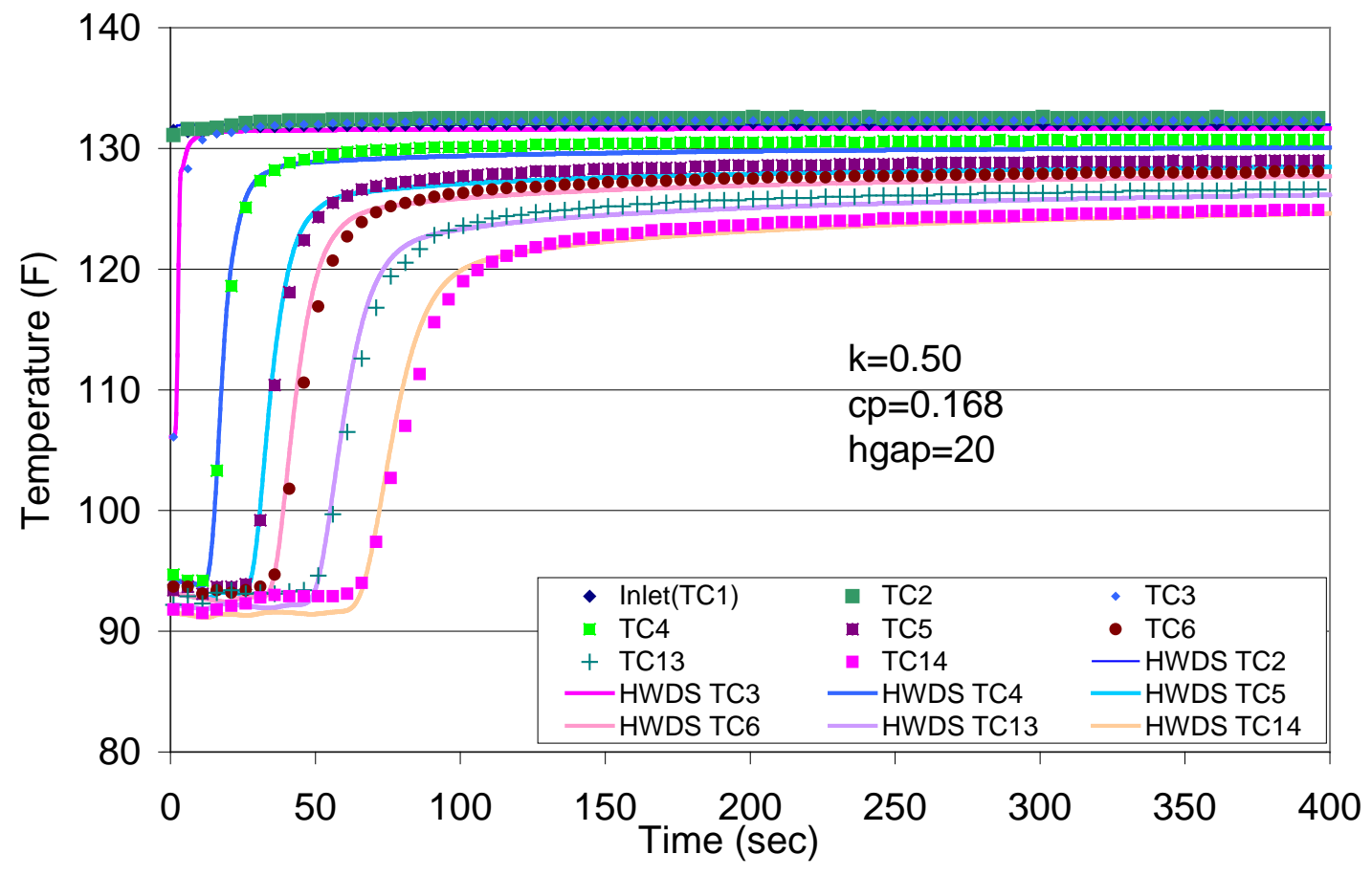



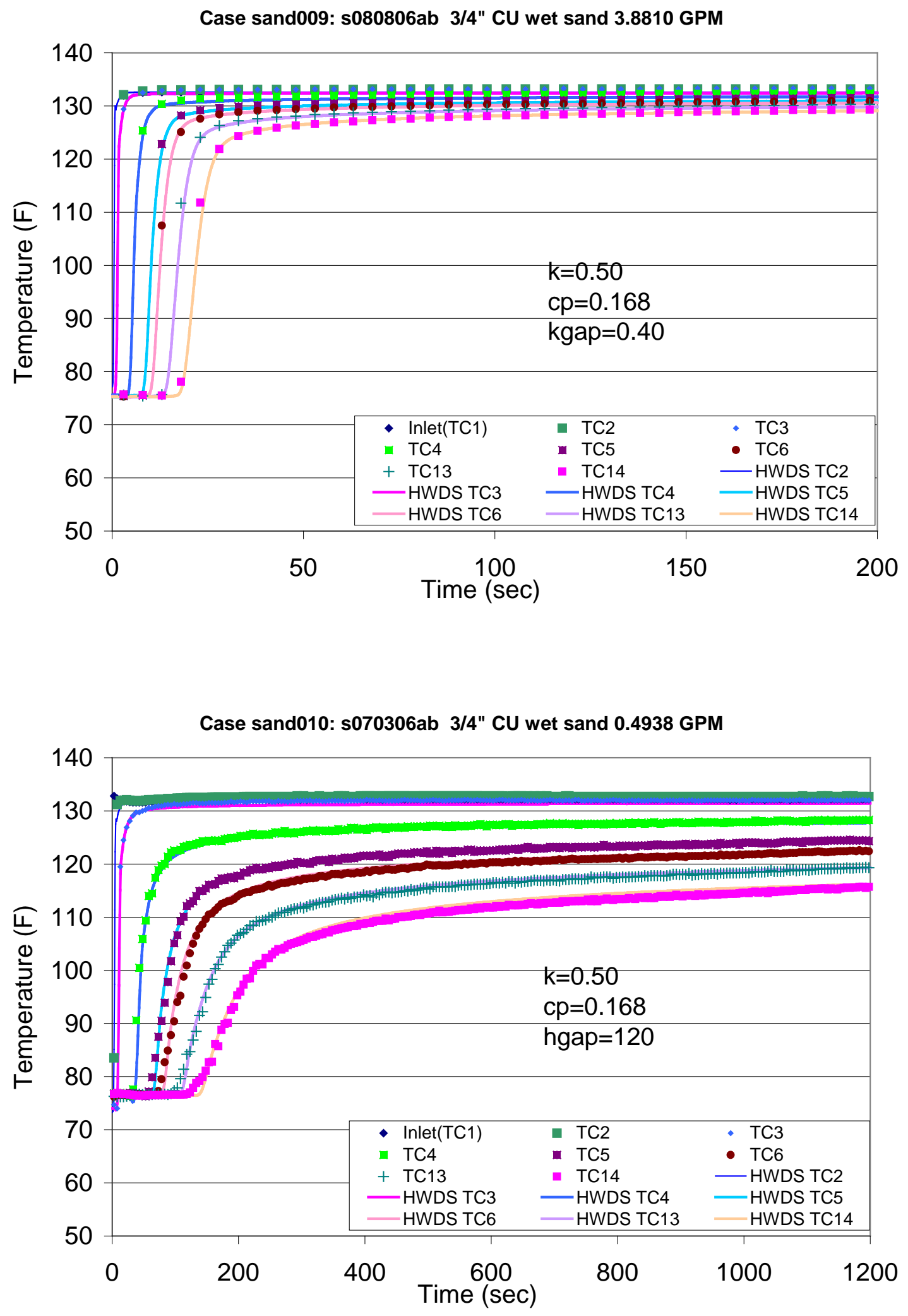


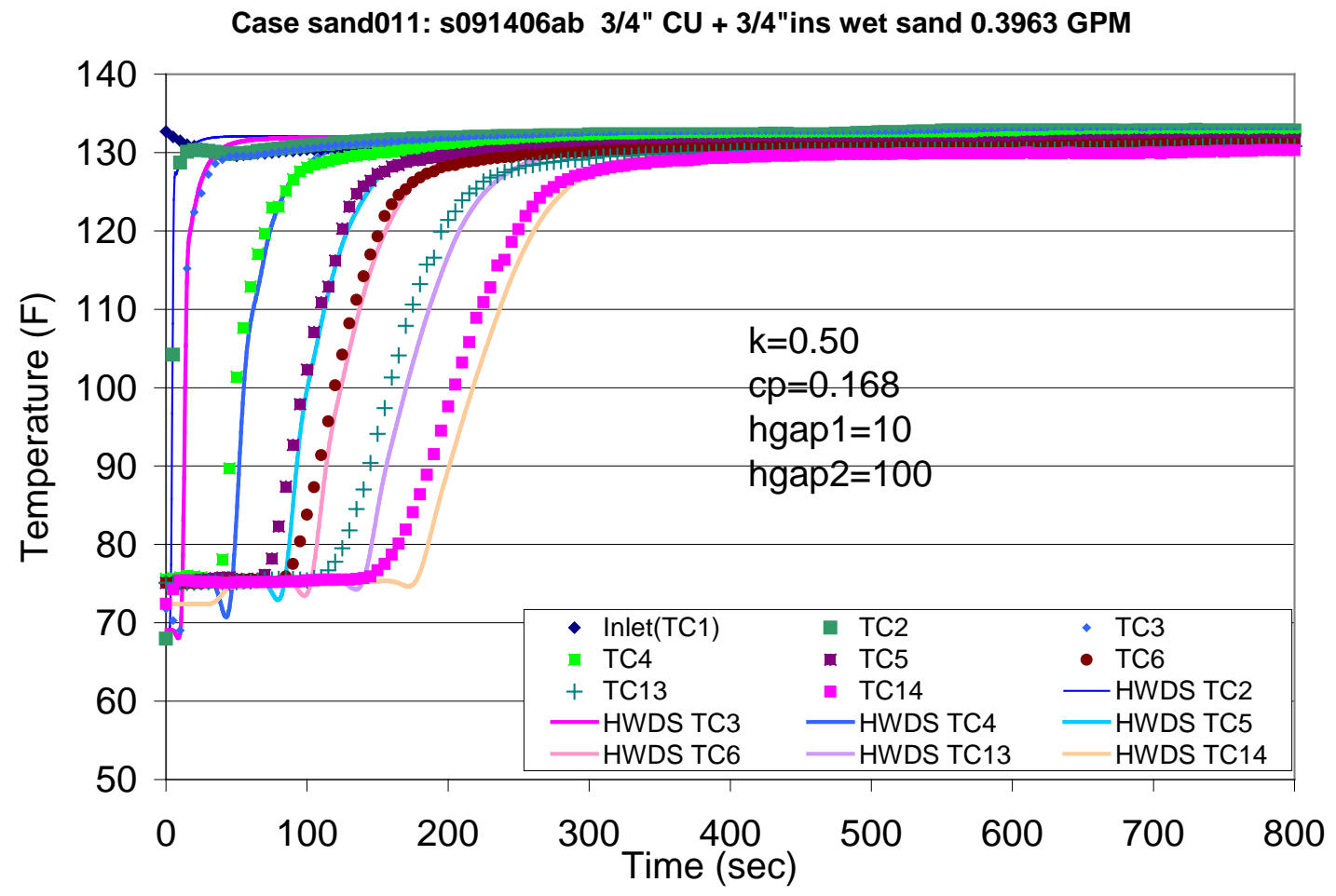

Case sand012: s091406ab 3/4" CU + 3/4"ins wet sand 1.1412 GPM

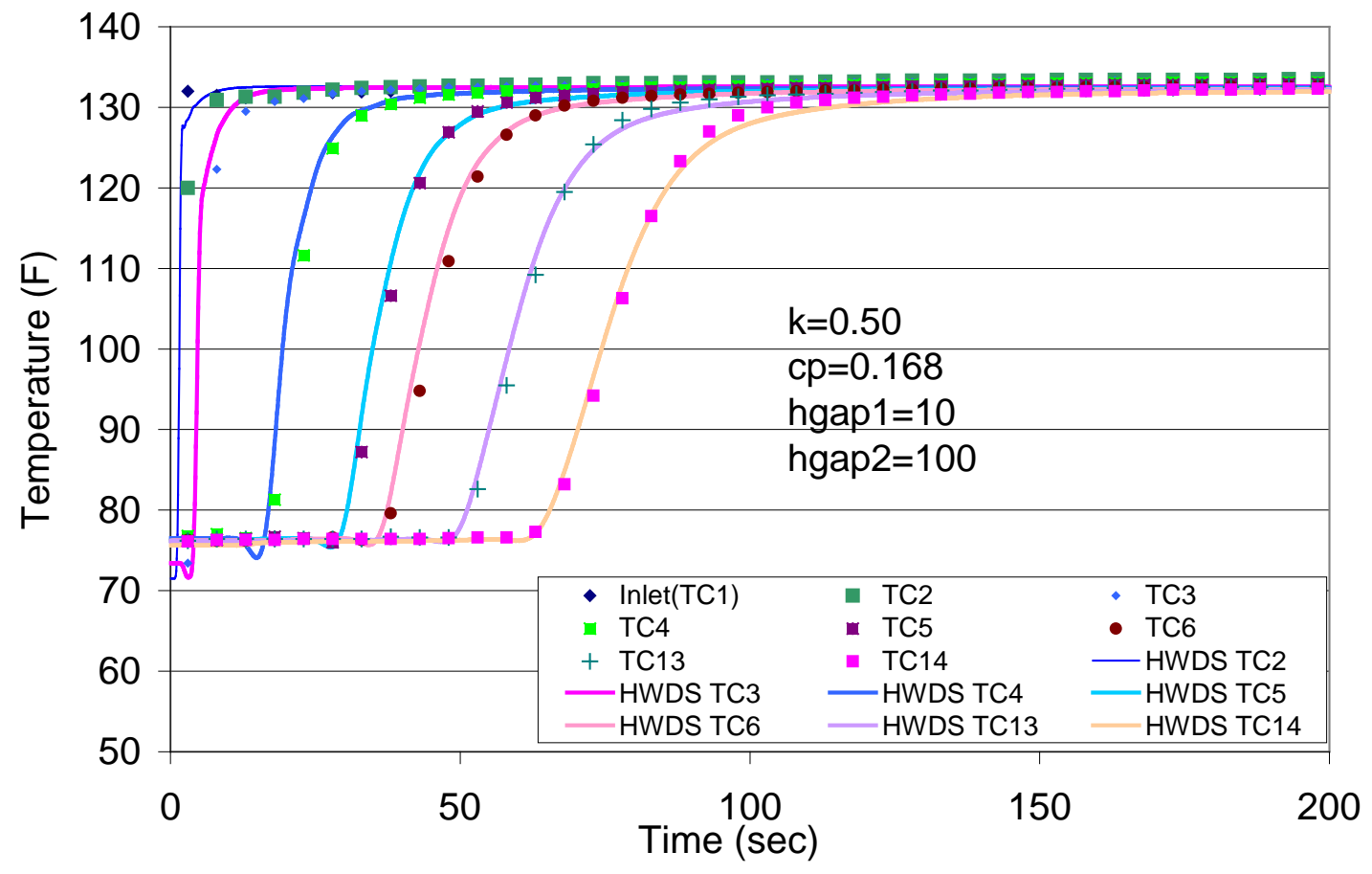



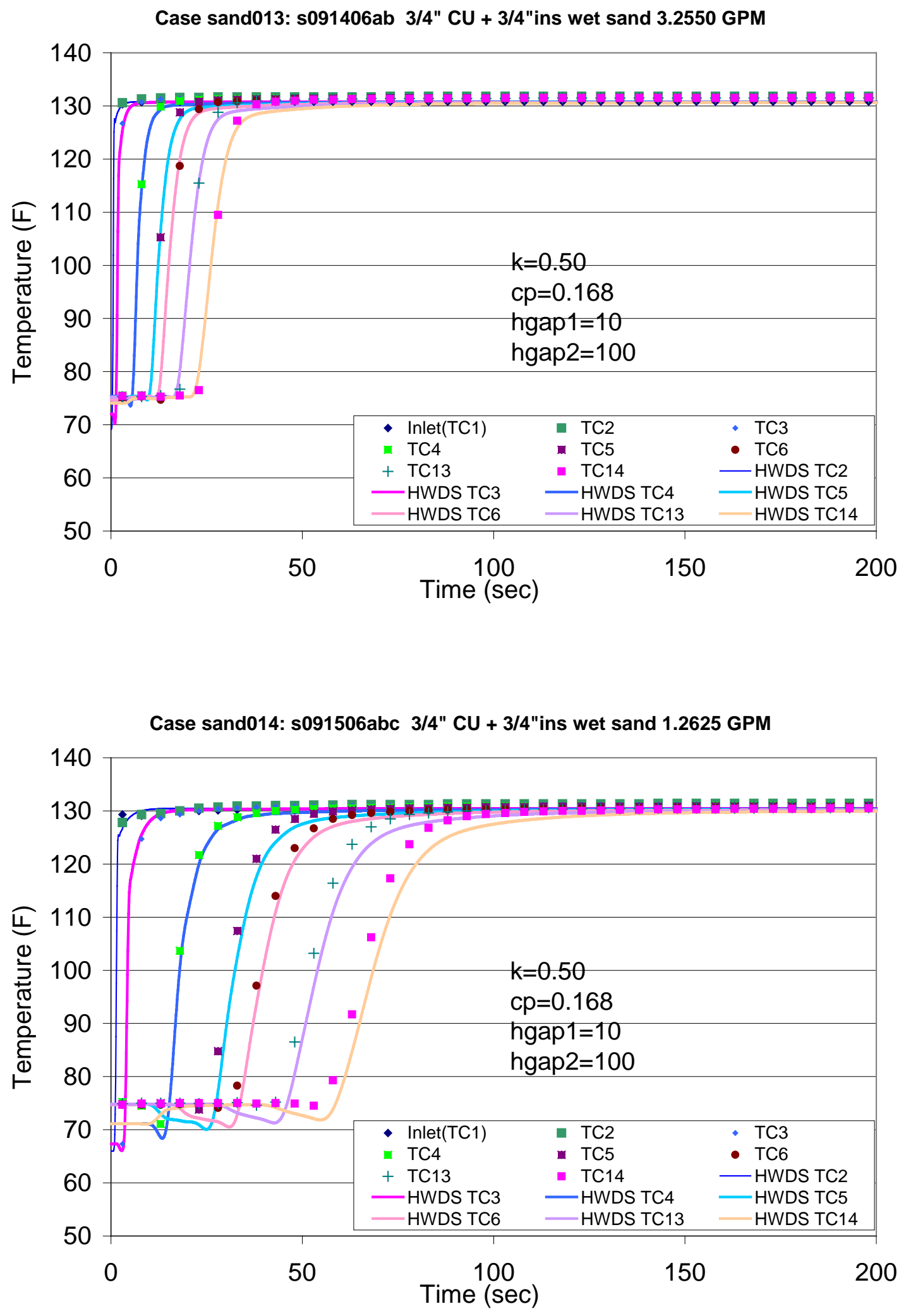

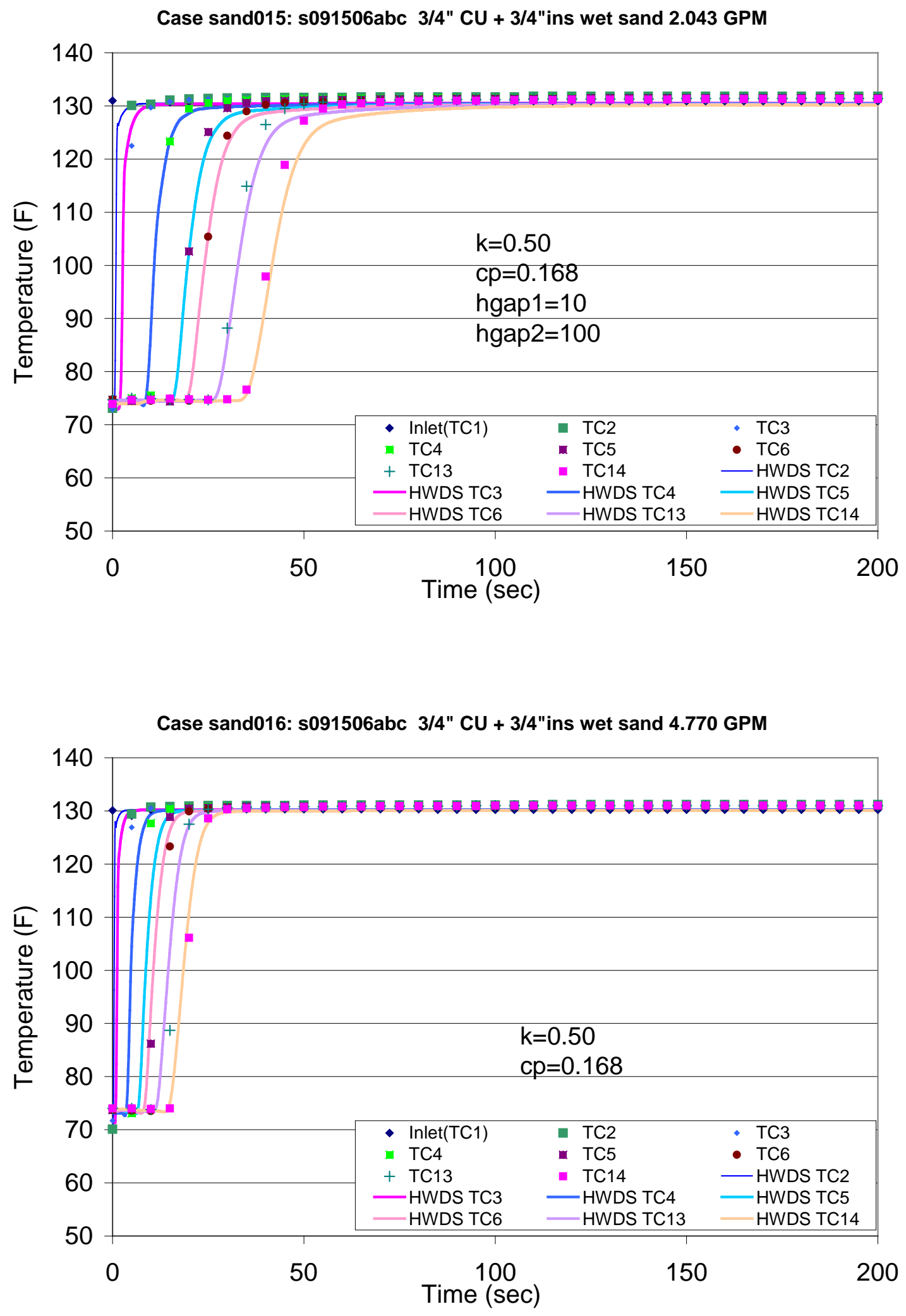

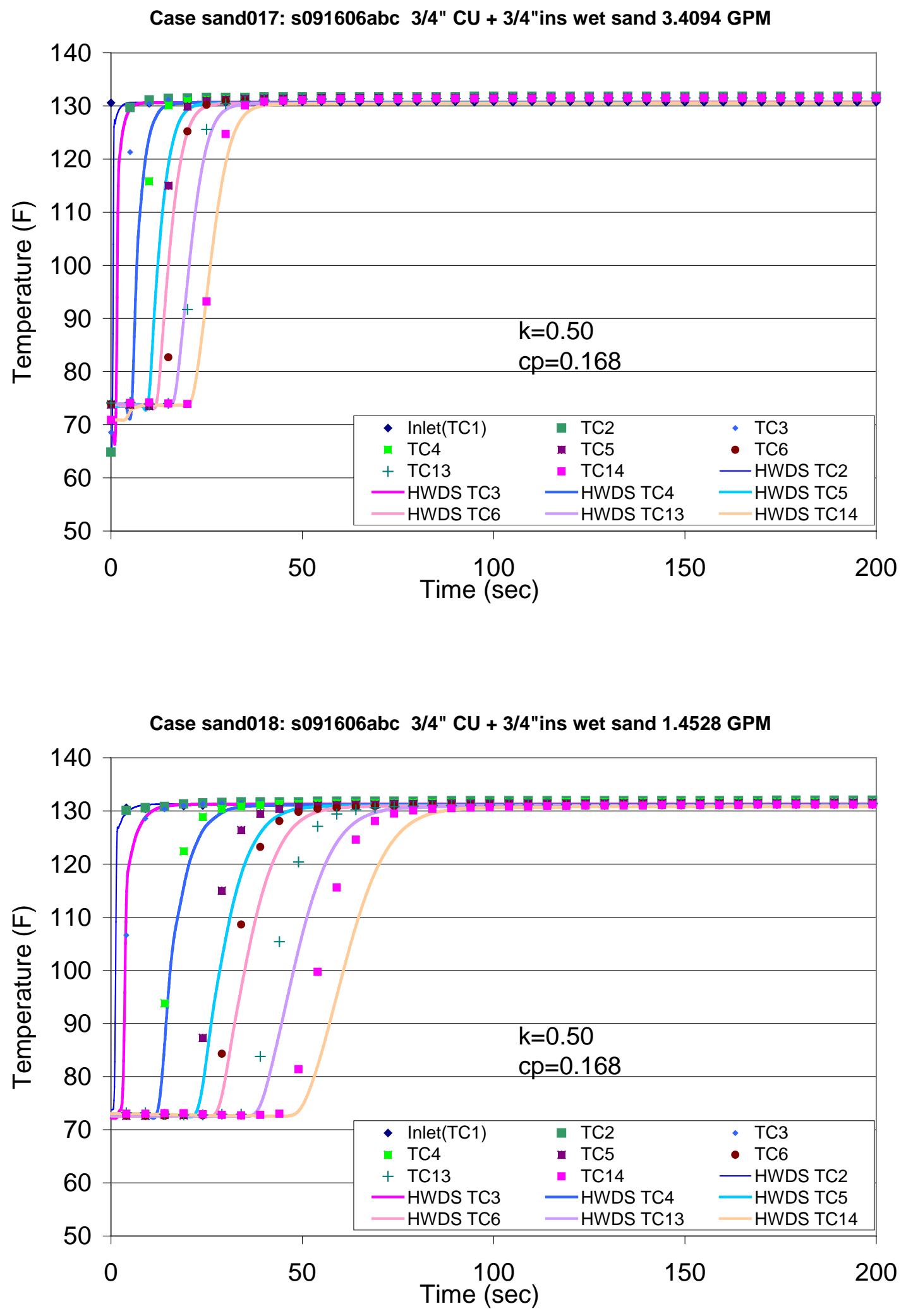

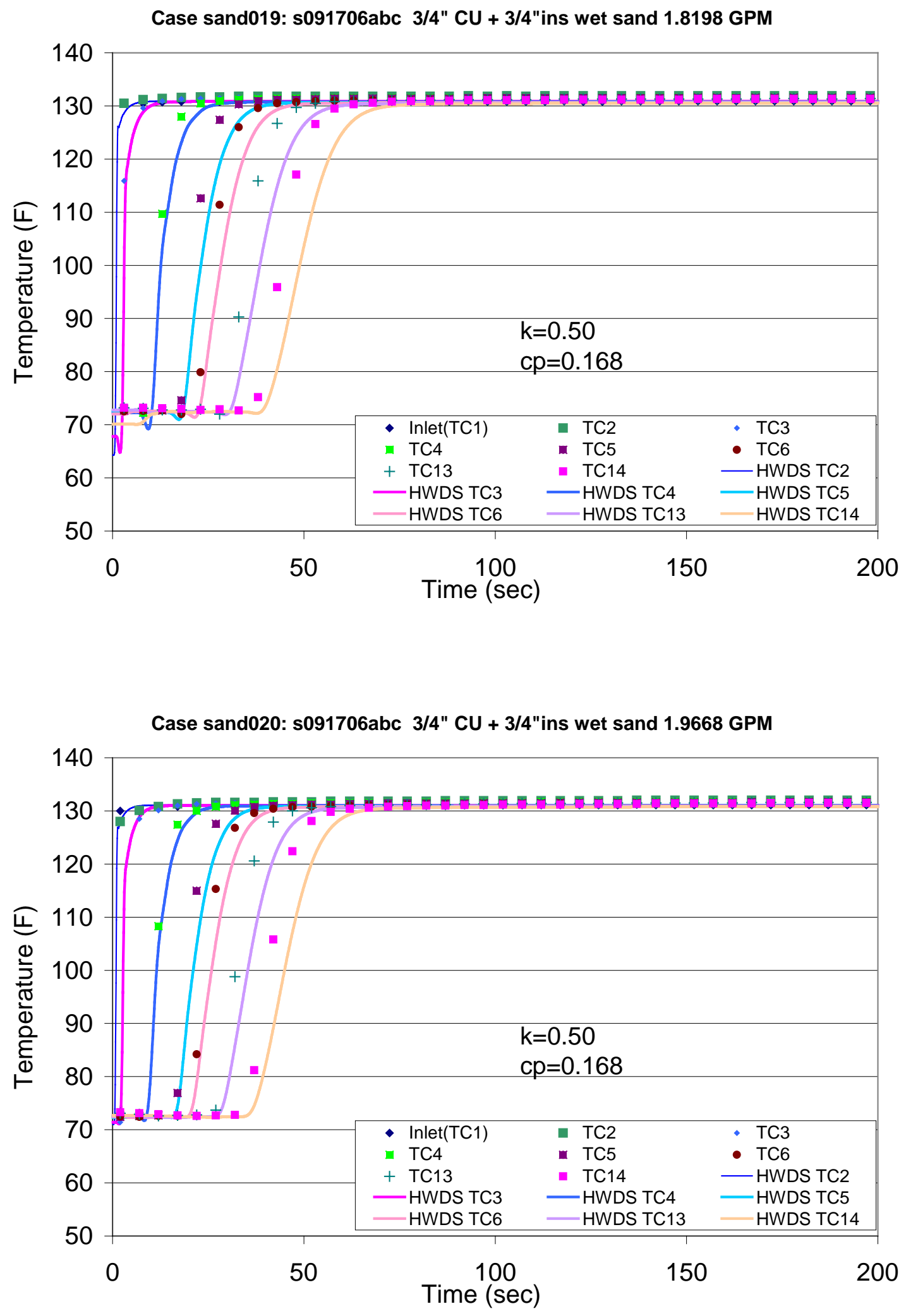


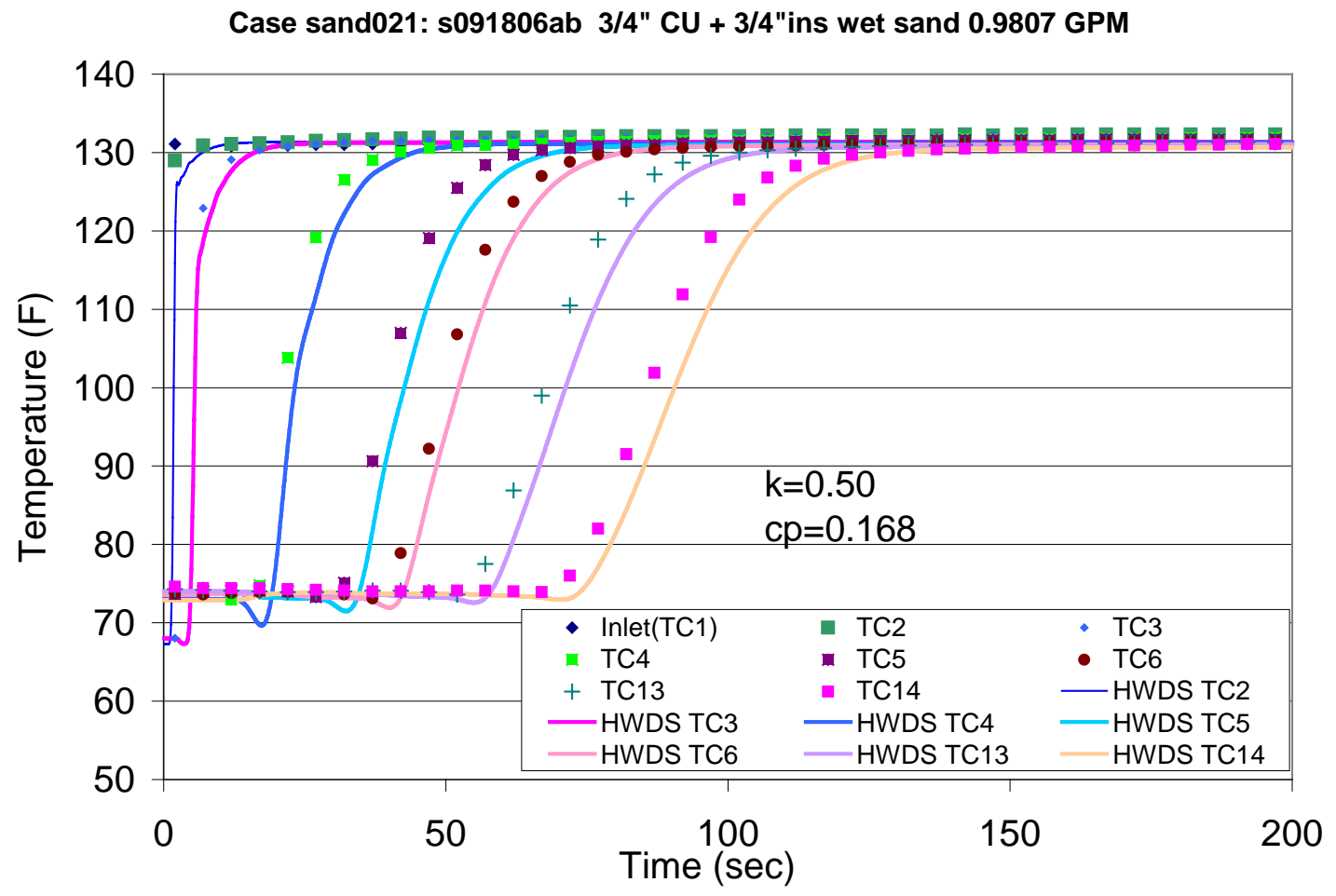





\section{Appendix E. MODIFICATIONS TO HWDS}

\section{MODIFICATION NOTES FOR VERSION 2.0 OF HWDS FOR ORNL}

1. In order to compare to the cases that Hiller has provided, need to be able to have different pipe materials for different segments (he has brass, rubber hose, and copper/pex in each run because of his experimental setup.

a) This can be done by modifying the 'PipeInfo[MAXEVENTS+1]' structure to a twodimensional array 'PipeInfo[MAXEVENTS+1][MAXSEGS+1]'.

b) The INPUT DATA FILE also will be modified. Now we expect one line for each segment with thermal properties k, rho, cp, and emiss. (the insulation properties are last, as before).

2. In order to compare to the cases that Hiller has provided, need to be able to have a draw schedule (flow rate versus time). The very first case I have has about 1.12 GPM for 120 secs, followed by 2.0 GPM for 60 seconds, followed by 0.6 GPM for 360 sec.

a) In order to do this, the 'flowrate[ MAXEVENTS+1 ]' variable must be made into a grid array like 'flowrate[ MAXEVENTS+1 ][MAXFLOWTIMES+1]', and an accompanying 'times_change_flow[MAXEVENTS+1][MAXFLOWTIMES+1]' implemented. The variable flowrate is then restricted to stepwise constant segments.

b) This requires insertion of a new input record with variable 'n_flow_times[MAXEVENTS + 1 ]' just before the flowrate. Now follow 'n_flow_times' records of 'flowrate_stop_time' pairs, where 'flowrate_stop_time' is the last time for which 'flowrate' applies.

c) Subsequently must change the 'Qflow' and 'mdot' variables in the routine 'SetupForTime(event, seg, PA.itime)' which is called at the top of the time integration loop.

d) To minimize function evaluations, added a variable 'current_flow_index' to keep track of which piece of the flow curve is current.

3. Added a new compile switch VERSION_20_INPUT which activates the changes indicated in 1. and 2. above.

4. Use compile switch VERSION_20_OUTPUT , add column of output for UA/L.

5. To accommodate a composite pipe material such as the "PAX" which has different thermal conductivity in the radial and axial direction, a matrix thermal conductivity must be used. (See additional development notes). Added new compile switch USE_COMPOSITE_K to swap this code in.

6. Added $\mathrm{k} \_r$ and $\mathrm{k} \_\mathrm{x}$ to the ElementInfo.h definition file.

7. Added $\mathrm{k} \_r$ and $\mathrm{k} \_\mathrm{x}$ to the ThermInfo structure in the PipeAll.h definition file.

8. Added k_r and k_x local variables in the Compute2DElements function in PipeAllTrans.cpp source file.

9. Added initialization in BuildElementInfoAll.c source file for $\mathrm{k} \_r$ and $\mathrm{k} \_x$. For 'pipe' use the input k_x and k_r; for "Ins" and "Environ" set $\mathrm{k} \_r$ and $\mathrm{k} \_x$ equal to ' $\mathrm{k}$ '.

10. Added pointers * $\mathrm{k} \_\mathrm{r}$ and $* \mathrm{k} \_\mathrm{x}$ into the call list for BuildElementInfoAll.

11. Added vectors and storage allocation for k_r_pipe and k_x_pipe in SetupForSeg in RunPipeAllTrans.cppp source module.

12. Added *k_r_pipe and $* \mathrm{k} \_\mathrm{x} \_$pipe pointers into PipeAll class definition. 
13. Added assignment of " $k \_$" and " $k \_x$ " for insulation and environment to the values of isotropic " $k$ " for the USE_COMPOSITE_K case.

14. Assigned " $k$ " to " $k \_r$ " for pipe to use in the "UA/L" calculation for the USE_COMPOSITE_K case.

15. modified grid generation to allow for shorter segments. Added definition for MINIMUM_SECTIONS_PER_SEG in the PipeAll.h file In function "SetupGrids" moved the "dx" initialization to top of "seg" loop and put "if" condition to ensure at least MINIMUM_SECTIONS_PER_SEG divisions per segment.

16. Patched a bug in the SetupGrids routine for sand/insulation case where the nominal ratios ratio1 and ratio2 need to be reset for each segment.

17. Added a compile time directive AXIAL_MIXING which should be set to a real number scalar (currently 1.0). This scalar multiplies the thermal conductivity of the fluid to simulate effects of axial mixing of the fluid.

18. Added a gap element between the pipe and insulation and pipe/insulation and surrounding sand. The heat flow through these gaps are controlled by two heat transfer coefficients (contact conductances).

19. Added two gap conductances hgap1 and hgap2 to the PipeAll class. These values are read at the end of the input file. If these values are not supplied (end of file found first), default values are used. ${ }^{* *}$ NOTE these values must be specified for multiple events in one file, but can be omitted for a single event per file.

20. Added HGAP1_DEFAULT and HGAP2_DEFAULT variables to PipeAll.h to set compile-time defaults. These are overwritten by input values, if supplied in the input file.

21. Added compiler switch USE_GAP_ELEMENTS to compile code which implements the gap elements. If this switch is \#undef'd, then perfect contact is used between radial elements.

22. Added debugging flag WRITE_RADIAL_DISTRIBUTION. When this flag is \#define'd, a second output file with the name "rad_"outputfile is created (where outputfile is the normal output file name specified on the command line) and the radial temperature distribution at some points along each segment are written at each time. 


\section{Appendix F. DEVELOPMENT NOTES}

\section{Non-isotropic pipe conductivity}

Here we repeat the analysis for the pipe elements but allow a composite structure with different conductivity in the radial and axial directions. IN particular, we assume that the heat flux vector is related to the temperature field as

$$
\vec{q}^{\prime \prime}=\left\{\begin{array}{l}
q_{x} \\
q_{r}
\end{array}\right\}=-\left[\begin{array}{cc}
k_{x} & 0 \\
0 & k_{r}
\end{array}\right]\left\{\begin{array}{l}
\frac{\partial T}{\partial x} \\
\frac{\partial T}{\partial r}
\end{array}\right\}
$$

The heat conduction equation in cylindrical coordinates can be written as

$$
\nabla \cdot \vec{q}^{\prime \prime}+\rho c_{p} \frac{\partial T}{\partial t}=0
$$

The weighted residual statement for an element is formed by multiplying by one of the interpolation functions and integrating over the area (volume) of the element:

$$
\int_{\Omega^{e}} N_{i}\left(\nabla \cdot \vec{q}^{\prime \prime}\right) d \Omega+\int_{\Omega^{e}} N_{i}\left(\rho c_{p} \frac{\partial T}{\partial t}\right) d \Omega=0
$$

Apply Green's theorem to the first term

$$
\int_{\Gamma^{e}} N_{i}\left(\vec{q}^{\prime \prime} \cdot \hat{n}\right) d \Gamma-\int_{\Omega^{e}} \nabla N_{i} \cdot \vec{q}^{\prime \prime} d \Omega+\int_{\Omega^{e}} N_{i}\left(\rho c_{p} \frac{\partial T}{\partial t}\right) d \Omega=0
$$

The term in parenthesis in the line integral is the heat flow out of the element. If the term is moved to the right side of the equation it is the heat flow into the element. Also, the heat flux vector is given by the Fourier Law $\vec{q}^{\prime \prime}=-[k]\{\nabla T\}$, so Eq. (3) can be written as

$$
\int_{\Omega^{e}} N_{i}\left(\rho c_{p} \frac{\partial T}{\partial t}\right) d \Omega+\int_{\Omega^{e}} \nabla N_{i} \cdot([k][\nabla T\}) d \Omega+=\int_{\Gamma^{e}} N_{i}\left(q_{i n}\right) d \Gamma
$$

For the two dimensional elements, a simple bi-linear interpolation function will be used on each

element:

$$
\left.T(r, x, t)=\left\lfloor N_{p}(r, x)\right\rfloor T_{p}(t)\right\}
$$




$$
\begin{aligned}
& N_{p 1}(r, x)=\left(1-\left(x-x_{1}\right) / \ell_{e}\right)\left(1-\left(r-r_{1}\right) / w_{e}\right) \\
& N_{p 2}(r, x)=\left(\left(x-x_{1}\right) / \ell_{e}\right)\left(1-\left(r-r_{1}\right) / w_{e}\right) \\
& N_{p 3}(r, x)=\left(\left(x-x_{1}\right) / \ell_{e}\right)\left(\left(r-r_{1}\right) / w_{e}\right) \\
& N_{p 4}(r, x)=\left(1-\left(x-x_{1}\right) / \ell_{e}\right)\left(\left(r-r_{1}\right) / w_{e}\right)
\end{aligned}
$$

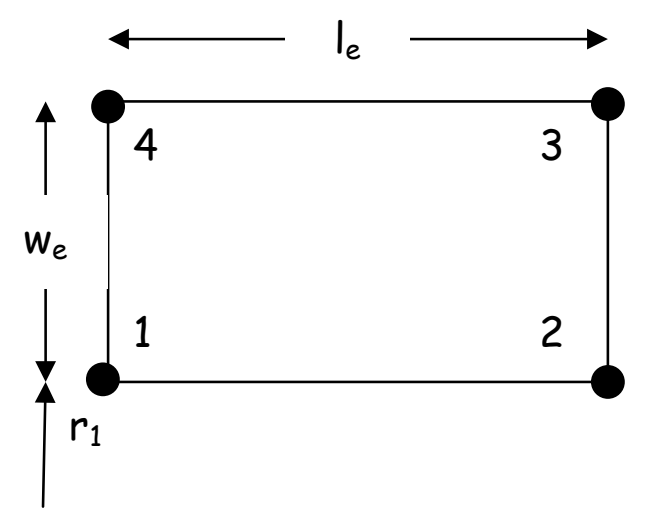

Fig. 2. Nomenclature for 2-D axisymmetric element.

Define the global derivative operator $\mathbf{B}$ as

$$
\mathbf{B}=\left[\begin{array}{ll}
\frac{\partial N_{i}}{\partial r} & \frac{\partial N_{i}}{\partial x}
\end{array}\right]^{T}
$$

and writing Eq. (4) for all four nodes ( $i=1,2,3$, 4), with the area integral $d \Omega=2 \pi r d r d x$

$$
2 \pi \int_{\Omega^{e}} \rho c_{p}\left\{N_{p}\right\} N_{p} \mid r d r d x\left\{\dot{T}_{p}\right\}+2 \pi \int_{\Omega^{e}} \mathbf{B}^{T}[k] \mathbf{B} r d r d x\left\{T_{p}\right\}=\int_{\Gamma^{e}}\left\{N_{p}\right\}\left(q_{i n}\right) d \Gamma
$$

In order to handle the $r$ in the integrand, we follow the suggestion of Pepper and Heinrich [4] regarding axisymmetric problems by interpolating the radial distance $r$ using the same basis functions as for the field variables. In this case, the $r$ obviously doesn't have any $x$ dependence, so the onedimensional version of the interpolation functions can be used:

$$
r^{e}=\left\lfloor 1-\left(r-r_{1}\right) / w_{e} \quad\left(r-r_{1}\right) / w_{e}\right\rfloor\left\{\begin{array}{l}
r_{1} \\
r_{2}
\end{array}\right\}
$$

Then Eq. (8) can be written as

$$
\left[C_{p}\right]\left\{\dot{T}_{p}\right\}+\left[K_{p}\right]\left\{T_{p}(t)\right\}=\left\{R_{p q}\right\}
$$


where

$$
\begin{aligned}
& {\left[C_{p}\right]=\rho c_{p} 2 \pi \int_{r_{1}}^{r_{2} \ell_{e}}\left\{N_{p}\right\} N_{p} \mid r^{e} d r d x} \\
& =\frac{2 \pi \rho c_{p} \ell_{e} w_{e}}{72}\left[\begin{array}{cccc}
6 r_{1}+2 r_{2} & 3 r_{1}+r_{2} & r_{1}+r_{2} & 2\left(r_{1}+r_{2}\right) \\
& 6 r_{1}+2 r_{2} & 2\left(r_{1}+r_{2}\right) & r_{1}+r_{2} \\
& & 2 r_{1}+6 r_{2} & r_{1}+3 r_{2} \\
\text { sym } & & & 2 r_{1}+6 r_{2}
\end{array}\right] \\
& {\left[K_{p}\right]=2 \pi \int_{r_{1}}^{r_{2} \ell_{e}} \int_{0}^{\mathbf{B}^{T}}[k] \mathbf{B} r^{e} d r d x} \\
& =\frac{2 \pi}{12 \ell_{e} w_{e}}\left[\begin{array}{cc}
4 k_{r} r_{1} w_{e}^{2}+k_{r} w_{e}^{3}+4 k_{x} \ell_{e}^{2} r_{1}+2 k_{x} \ell_{e}^{2} w_{e} & -4 k_{r} r_{1} w_{e}^{2}-k_{r} w_{e}^{3}+2 k_{x} \ell_{e}^{2} r_{1}+k_{x} \ell_{e}^{2} w_{e} \\
& 4 k_{r} r_{1} w_{e}^{2}+k_{r} w_{e}^{3}+4 k_{x} \ell_{e}^{2} r_{1}+2 k_{x} \ell_{e}^{2} w_{e} \ldots \\
\operatorname{sym} &
\end{array}\right. \\
& -\left(k_{r} w_{e}^{2}+k_{x} \ell_{e}^{2}\right)\left(2 r_{1}+w_{e}\right) \quad\left(k_{r} w_{e}^{2}-2 k_{x} \ell_{e}^{2}\right)\left(2 r_{1}+w_{e}\right) \\
& \left(k_{r} w_{e}^{2}-2 k_{x} \ell_{e}^{2}\right)\left(2 r_{1}+w_{e}\right) \quad-\left(k_{r} w_{e}^{2}+k_{x} \ell_{e}^{2}\right)\left(2 r_{1}+w_{e}\right) \\
& { }^{\cdots} 4 k_{r} r_{1} w_{e}^{2}+3 k_{r} w_{e}^{3}+4 k_{x} \ell_{e}^{2} r_{1}+2 k_{x} \ell_{e}^{2} w_{e}-4 k_{r} r_{1} w_{e}^{2}-3 k_{r} w_{e}^{3}+2 k_{x} \ell_{e}^{2} r_{1}+k_{x} \ell_{e}^{2} w_{e} \\
& 4 k_{r} r_{1} w_{e}^{2}+3 k_{r} w_{e}^{3}+4 k_{x} \ell_{e}^{2} r_{1}+2 k_{x} \ell_{e}^{2} w_{e}
\end{aligned}
$$

If, at the inner pipe boundary, the heat flux from the fluid to the pipe is described by the Eq. (2), then there are two terms:

$$
\begin{gathered}
{\left[K_{h}\right]=\int_{0}^{\ell_{e}} p h(x, t)\{N\}\lfloor N]\left\{T^{e}(t)\right\} d x=p \frac{h_{e l e} \ell_{e}}{6}\left[\begin{array}{llll}
2 & 1 & 0 & 0 \\
1 & 2 & 0 & 0 \\
0 & 0 & 0 & 0 \\
0 & 0 & 0 & 0
\end{array}\right]} \\
\left\{R_{h}\right\}=\int_{0}^{\ell_{e}} p h(x, t)\{N\} T_{f}(x, t) d x \\
\left.=p \frac{h_{e l e} \ell_{e}}{6}\left[\begin{array}{llll}
2 & 1 & 0 & 0 \\
1 & 2 & 0 & 0 \\
0 & 0 & 0 & 0 \\
0 & 0 & 0 & 0
\end{array}\right]\left\{\begin{array}{c}
T_{f 1} \\
T_{f 2} \\
0 \\
0
\end{array}\right\}=\left[K_{h}\right]\right\}\left\{\begin{array}{c}
T_{f 1} \\
T_{f 2} \\
0 \\
0
\end{array}\right\}
\end{gathered}
$$

In Eq. (11d), the $T_{f 1}$ and $T_{f 2}$ are the temperatures of the fluid corresponding to the nodes 1 and 2 for the two-dimensional element, and $T_{f}(x, t)$ has been interpolated linearly along the length of the element. 
For the outer boundary, a type 3 condition, similar to Eqs. (11c-d), is given

$$
\begin{aligned}
& {\left[K_{h o}\right]=\int_{0}^{\ell_{e}} p h_{o}(x, t)\{N\} N \mid\left\{T^{e}(t)\right\} d x=p \frac{h_{o_{-} e l e} \ell_{e}}{6}\left[\begin{array}{llll}
0 & 0 & 0 & 0 \\
0 & 0 & 0 & 0 \\
0 & 0 & 2 & 1 \\
0 & 0 & 1 & 2
\end{array}\right] } \\
&\left\{R_{q h}\right\}=\int_{0}^{\ell_{e}} p h_{o}(x, t)\{N\} T_{\infty}(x, t) d x \\
&=p \frac{h_{o_{-} e l e} \ell_{e}}{6}\left[\begin{array}{llll}
0 & 0 & 0 & 0 \\
0 & 0 & 0 & 0 \\
0 & 0 & 2 & 1 \\
0 & 0 & 1 & 2
\end{array}\right]\left\{\begin{array}{c}
0 \\
0 \\
T_{\infty 1} \\
T_{\infty 2}
\end{array}\right\}
\end{aligned}
$$

\section{Gap Elements}

To account for the effect of an imperfect contact, a "gap element" is implemented which decouples the external domain from the internal one. Thermal communication between the two domains is facilitated by a heat transfer coefficient (thermal contact conductance) across the gap. This results in an extra "row" of solution variables since the temperature on both sides of the "gap" must now be computed.
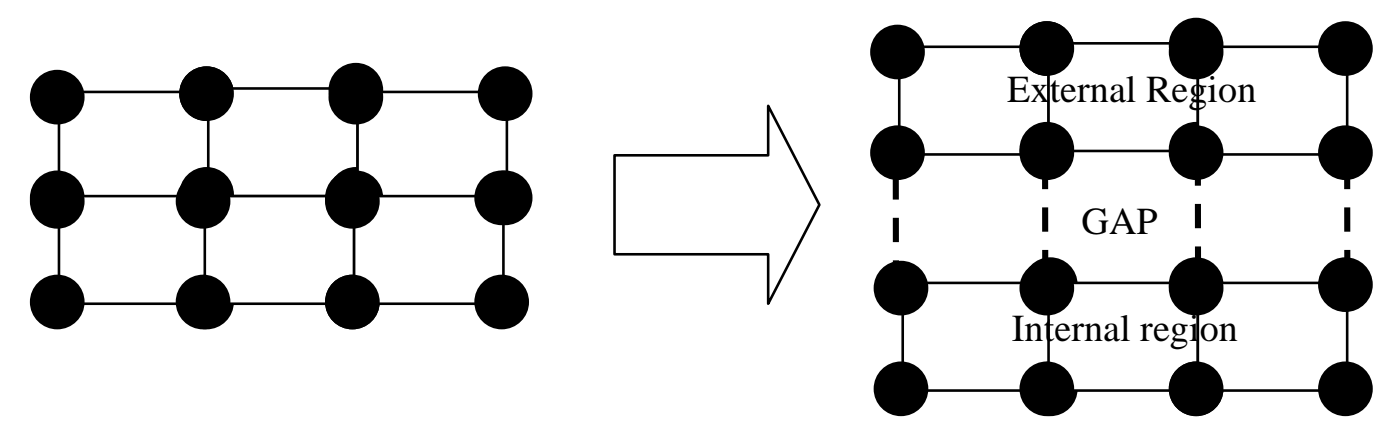

Required modifications:

1. New degrees of freedom need to be assigned for the internal edge elements. The external edge elements will assume the degrees of freedom prior to the gap implementation.

2. Heat transfer coefficient boundary condition modifications must be made along the two edges (this requires modification of the stiffness matrix for these elements). This can be handled through the connectivity information for the "gap element"

3. Make an element for the gap in any case. Assign r1 = r2 for this element. "we" will be zero and will give a zero divide in $2 \mathrm{~d}$ elements so block this. Assembly process as usual adds nothing to global matrix and in fact leaves it singular. Beauty of this approach is that now the "einfo" for this gap element has the connectivity information needed for the modification. 


\section{Modifications}

1. in SetupGrids, if insThick $>0$, added extra entry into the rvec array with same radius as pipe to facilitate gap element

2. in SetupGrids, if thick $>0$, added extra entry into the rvec array to facilitate gap.

3. In SetupGrids, added check to increment rvec_count if ndr2 $>0$ and also again if ndr3 $>0$

4. In compute2Delements, added check to skip if we $=0$

5. Added CMatrix Tcurrent to the PipeAll class to have access to solution variables for iterations.

6. In RunPipeAllTrans add call to Apply_hgap immediately following Apply_ho

This all seems to be working — next add "straightwire” to make gap elements behave for the "no gap" case...

For the "no gap" case, set the temperature on outside of gap equal to the temperature on the inside of gap and iterate.

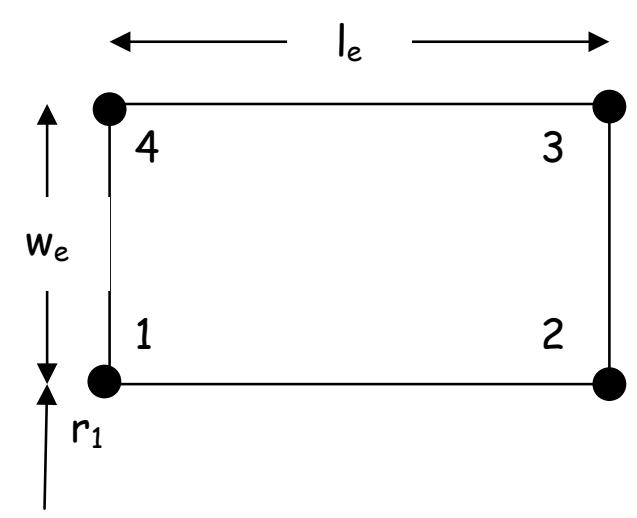

Fig. - Nomenclature for 2-D axisymmetric element.

Note for gap element $r 1=r 2$ and we $=0$. for "No gap", $T_{1}=T_{4}$ and $T_{2}=T_{3}$. We set this up as an equation for the element that says:

$$
\left[\begin{array}{llll}
0 & 0 & 0 & 0 \\
0 & 0 & 0 & 0 \\
0 & 0 & 1 & 0 \\
0 & 0 & 0 & 1
\end{array}\right]\left\{\begin{array}{l}
T_{1} \\
T_{2} \\
T_{3} \\
T_{4}
\end{array}\right\}=\left\{\begin{array}{c}
0 \\
0 \\
T_{1} \\
T_{2}
\end{array}\right\}
$$

where $T_{1}$ and $T_{2}$ are the temperatures from the inner portion for the previous iteration. 


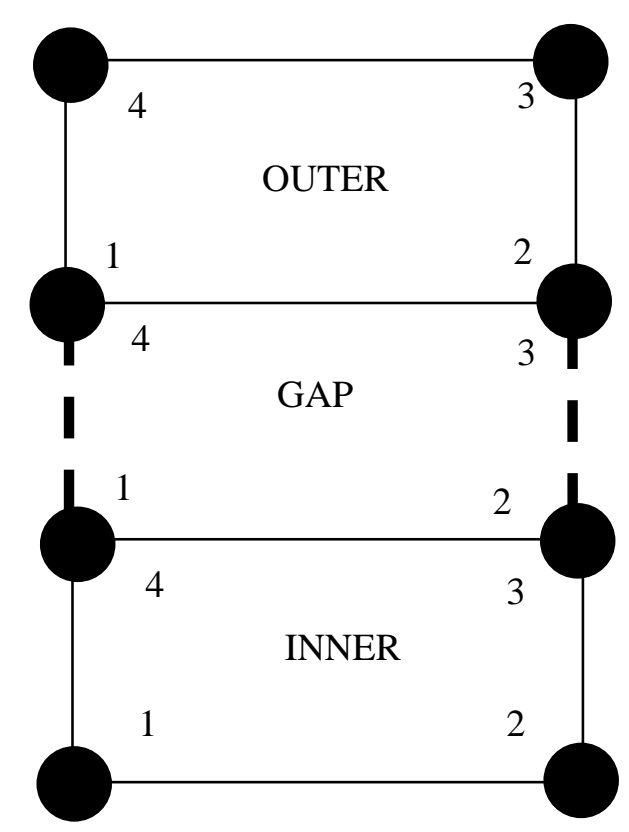

Fig. - Gap Element and relation to inner and outer regions

Note that global DOFs for the INNER $3+4$ are same as GAP $1+2$

Note that global DOFs for the OUTER $1+2$ are same as GAP 3+4

We don't need any equations for the GAP element itself, but use the connectivity of the GAP element to

1. Apply convective boundary condition to INNER element-modifying nodes $1+2$ in GAP (and therefore global DOFs for INNER 3+4) and tying them to GAP 3+4 (therefore OUTER $1+2$ global DOFs)

2. Also apply convective boundary condition to OUTER element-just use the GAP connectivity

3. Should be able to put all this into a matrix equation/constraint for the gap

$$
\left[K_{h}\right]\left\{T_{\text {ele }}\right\}=\left\{R_{q h}\right\}
$$




$$
\begin{aligned}
& p \frac{h_{o_{-} \text {ele }} \ell_{e}}{6}\left[\begin{array}{llll}
0 & 0 & 0 & 0 \\
0 & 0 & 0 & 0 \\
0 & 0 & 2 & 1 \\
0 & 0 & 1 & 2
\end{array}\right]\left\{\begin{array}{l}
T_{G A P-1} \\
T_{G A P-2} \\
T_{G A P-3} \\
T_{G A P-4}
\end{array}\right\}=p \frac{h_{o_{-} e l e} \ell_{e}}{6}\left[\begin{array}{llll}
0 & 0 & 0 & 0 \\
0 & 0 & 0 & 0 \\
0 & 0 & 2 & 1 \\
0 & 0 & 1 & 2
\end{array}\right]\left\{\begin{array}{c}
0 \\
0 \\
T_{G A P-2} \\
T_{G A P-1}
\end{array}\right\} \\
& p \frac{h_{o_{-} e l e} \ell_{e}}{6}\left[\begin{array}{llll}
0 & 0 & 0 & 0 \\
0 & 0 & 0 & 0 \\
0 & 0 & 2 & 1 \\
0 & 0 & 1 & 2
\end{array}\right]\left\{\begin{array}{l}
T_{G A P-1} \\
T_{G A P-2} \\
T_{G A P-3} \\
T_{G A P-4}
\end{array}\right\}=p \frac{h_{o_{-} e l e} \ell_{e}}{6}\left[\begin{array}{llll}
0 & 0 & 0 & 0 \\
0 & 0 & 0 & 0 \\
1 & 2 & 0 & 0 \\
2 & 1 & 0 & 0
\end{array}\right]\left\{\begin{array}{l}
T_{G A P-1} \\
T_{G A P-2} \\
T_{G A P-3} \\
T_{G A P-4}
\end{array}\right\} \\
& p \frac{h_{o_{-} \text {ele }} \ell_{e}}{6}\left[\begin{array}{rrrr}
0 & 0 & 0 & 0 \\
0 & 0 & 0 & 0 \\
-1 & -2 & 2 & 1 \\
-2 & -1 & 1 & 2
\end{array}\right]\left\{\begin{array}{l}
T_{G A P-1} \\
T_{G A P-2} \\
T_{G A P-3} \\
T_{G A P-4}
\end{array}\right\}=0
\end{aligned}
$$

It should be a full matrix...

$$
\begin{aligned}
& p \frac{h_{o_{-} e l e} \ell_{e}}{6}\left[\begin{array}{llll}
2 & 1 & 0 & 0 \\
1 & 2 & 0 & 0 \\
0 & 0 & 2 & 1 \\
0 & 0 & 1 & 2
\end{array}\right]\left\{\begin{array}{l}
T_{G A P-1} \\
T_{G A P-2} \\
T_{G A P-3} \\
T_{G A P-4}
\end{array}\right\}=p \frac{h_{o_{-} e l e} \ell_{e}}{6}\left[\begin{array}{llll}
2 & 1 & 0 & 0 \\
1 & 2 & 0 & 0 \\
0 & 0 & 2 & 1 \\
0 & 0 & 1 & 2
\end{array}\right]\left\{\begin{array}{l}
T_{G A P-4} \\
T_{G A P-3} \\
T_{G A P-2} \\
T_{G A P-1}
\end{array}\right\} \\
& p \frac{h_{o_{-} e l e} \ell_{e}}{6}\left[\begin{array}{llll}
2 & 1 & 0 & 0 \\
1 & 2 & 0 & 0 \\
0 & 0 & 2 & 1 \\
0 & 0 & 1 & 2
\end{array}\right]\left\{\begin{array}{l}
T_{G A P-1} \\
T_{G A P-2} \\
T_{G A P-3} \\
T_{G A P-4}
\end{array}\right\}=p \frac{h_{o_{-} e l e} \ell_{e}}{6}\left[\begin{array}{llll}
0 & 0 & 1 & 2 \\
0 & 0 & 2 & 1 \\
1 & 2 & 0 & 0 \\
2 & 1 & 0 & 0
\end{array}\right]\left\{\begin{array}{l}
T_{G A P-1} \\
T_{G A P-2} \\
T_{G A P-3} \\
T_{G A P-4}
\end{array}\right\} \\
& p \frac{h_{o_{-} e l e} \ell_{e}}{6}\left[\begin{array}{rrrr}
2 & 1 & -1 & -2 \\
1 & 2 & -2 & -1 \\
-1 & -2 & 2 & 1 \\
-2 & -1 & 1 & 2
\end{array}\right]\left\{\begin{array}{l}
T_{G A P-1} \\
T_{G A P-2} \\
T_{G A P-3} \\
T_{G A P-4}
\end{array}\right\}=0
\end{aligned}
$$

